\title{
Blossoming of Coiled Deployable Booms
}

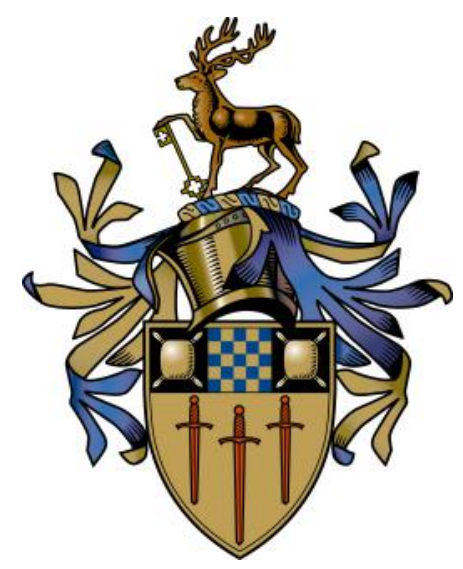

Adam Hoskin

Surrey Space Centre

University of Surrey

March 7, 2018

Submitted for the Degree of Doctor of Philosophy from the University of Surrey

Primary Supervisor: Professor Guglielmo S. Aglietti

Secondary Supervisors: Dr. Andrew Viquerat, Dr. Mark Schenk, Dr. Olive Stohlman 


\section{Acknowledgements}

I would like to thank all of my supervisors for their help and advice over the course of my PhD. I would also like to thank the technicians in the faculty and in the University workshop as well as my fellow PhD students at the Surrey Space Centre. Finally I would like to thank my Parents for the support they have given me throughout my education. 


\begin{abstract}
Coiled deployable booms have been used extensively in space and are a large part of the deployable space structures family. They have a wide variety of uses such as the deployment of instruments, gravity-gradient stabilisation masses and more recently solar sails. Most deployable booms are similar to a carpenter's tape measure in the way they are coiled in a retracted condition and then deploy to form the boom structure. There have been many developments in the optimisation of boom properties in the deployed state, by using different shape cross sections and by using different materials. The first metal tape spring booms have developed into the more modern booms with a variety of cross sections. One aspect that is common to all booms is the coiling and uncoiling process and the difficulties associated with this. Blossoming, where the boom starts to uncoil within the boom deployer, can lead to the jamming of the mechanism. The reasons behind blossoming have not been thoroughly investigated, leaving designers of booms, and boom housing mechanisms to try and mitigate this problem themselves, often by trial and error. This work investigates boom blossoming with the aim of better understanding the underlying mechanics so that more effective deployment systems can be designed in the future.

A method is developed that uses the strain energy stored in coiled booms to find the maximum tip force that can be achieved before blossoming occurs. This method is also used to investigate the central spindle torque during blossoming. The effects that the coil geometry and the friction between the layers of the coiled booms have on blossoming are also investigated.

The theory developed should enable the designers of tape spring deployers to estimate the tip force and central spindle torque of a tape spring boom in the design phase of projects and reduce the reliance on trial and improvement type testing once deployers have already been built.
\end{abstract}




\section{Contents}

1 Background $\quad 6$

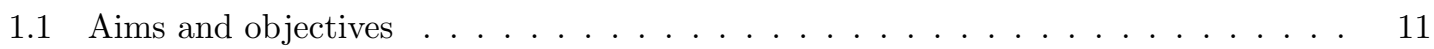

1.2 Elements of novelty . . . . . . . . . . . . . . . . . . . . 11

1.3 List of contributions . . . . . . . . . . . . . . . . . . . 11

1.4 Outline of report . . . . . . . . . . . . . . . . . . . 12

2 Introduction $\quad 13$

2.1 Solar sailing and drag deorbiting . . . . . . . . . . . . . . . . 13

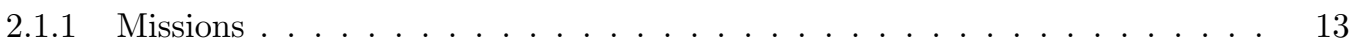

2.1 .2 Types of solar sail . . . . . . . . . . . . . . . . . . . 14

2.1.3 Current demonstrator missions . . . . . . . . . . . . . . 16

2.1 .4 Drag deorbiting . . . . . . . . . . . . . . . . . . 17

2.2 Coiled deployable booms . . . . . . . . . . . . . . . . . . . . . . . 17

2.3 CubeSail deployment process . . . . . . . . . . . . . . . . . . . 21

2.3 .1 Sail forces . . . . . . . . . . . . . . . . . 21

2.3 .2 CubeSail deployment . . . . . . . . . . . . . . . . 21

2.3.3 Blossoming ........................ 25

3 Energy method for tip force $\quad 28$

3.1 Strain energy in a tape spring bend . . . . . . . . . . . . . . . . . . 28

3.2 Constant radius method . . . . . . . . . . . . . . . . . . 33

3.2.1 Compression spring analogy . . . . . . . . . . . . . . 35

3.2 .2 Energy and force plots . . . . . . . . . . . . . . . . 36

3.2.3 Simple experimental test set-up . . . . . . . . . . . . . . . 38

3.3 Spiral geometry . . . . . . . . . . . . . . . . . . . . . . 40

3.4 Addition of compression springs . . . . . . . . . . . . . . . . . . 45

3.5 Experimental test set-up . . . . . . . . . . . . . . . . . . . . . 47

3.6 Results. . . . . . . . . . . . . . . . . . . . . . . . . . 48

3.7 Energy method conclusion . . . . . . . . . . . . . . . . 56

4 Central spindle and self-deployment torque $\quad 58$

4.1 Torque and force balance . . . . . . . . . . . . . . . . . . . 58

4.2 Self-deployment torque . . . . . . . . . . . . . . . . . . 60

4.3 Central spindle torque . . . . . . . . . . . . . . . . . . 61

4.4 Central spindle torque and self-deployment torque comparison . . . . . . . . . . 63

4.5 Zero central spindle torque . . . . . . . . . . . . . . . . . . . 64

4.6 Spiral geometry . . . . . . . . . . . . . . . . . . . . . . . 67

4.7 Experiments . . . . . . . . . . . . . . . . . . . 67

4.8 Central spindle and self-deployment torque conclusion . . . . . . . . . . . . . 73 


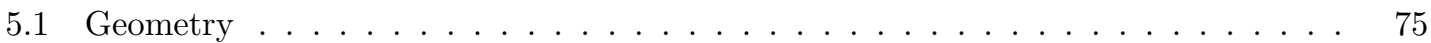

5.2 Friction force . . . . . . . . . . . . . . . . . . . 78

5.3 Results.............................. 82

6 Experimental test set-up $\quad 89$

6.1 Overview of experimental test set-up . . . . . . . . . . . . . . . . . 89

6.1 .1 Central spindle . . . . . . . . . . . . . . . . 9 90

6.1.2 Central spindle torque sensor and motor . . . . . . . . . . . . . . 91

6.1 .3 Tip force sensors . . . . . . . . . . . . . . . . . 91

6.1.4 Compression roller arms . . . . . . . . . . . . . . . 92

6.2 Details of experimental method . . . . . . . . . . . . . . . . . 93

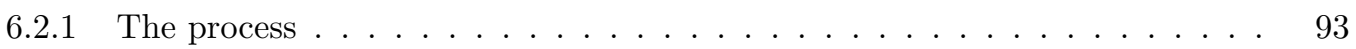

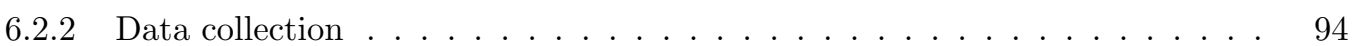

7 Conclusion $\quad 95$

8 Appendix A $r$

9 Appendix B $r 104$ 


\section{Nomenclature}

\begin{tabular}{|c|c|}
\hline$\Delta v$ & Change in velocity \\
\hline$\kappa_{x}$ & Curvature along the length of the boom \\
\hline$\kappa_{y}$ & Curvature across the width of the boom \\
\hline$\nu$ & Possion's ratio \\
\hline$\phi$ & Angle rotated by central spindle \\
\hline$\psi$ & Angle rotated by point within coil \\
\hline$\theta$ & Angle subtended by coil \\
\hline$A$ & Area of coiled part of tape spring \\
\hline$a$ & Tape spring thickness divided by $2 \pi$ \\
\hline$b$ & Tape spring width \\
\hline$C_{f}$ & Coefficient of friction \\
\hline$D$ & Flexural rigidity \\
\hline$E$ & Young's modulus \\
\hline$F$ & Force \\
\hline$F_{n}$ & Normal force \\
\hline$F_{c r}$ & Compression roller force \\
\hline$F_{t i p}$ & Tip force \\
\hline$I$ & Second moment of area of boom \\
\hline$k$ & Spring stiffness \\
\hline$L$ & Length of boom \\
\hline$m_{0}$ & Initial mass \\
\hline$m_{f}$ & Final mass \\
\hline$M_{e q}$ & Moment for equal sense bending \\
\hline$M_{o p}$ & Moment for opposite sense bending \\
\hline$N_{c}$ & Number of turns in the coil \\
\hline$N$ & Number of contact areas \\
\hline
\end{tabular}




\begin{tabular}{ll}
$N_{c r}$ & Number of compression rollers \\
$Q$ & Coil number from outside of boom coil \\
$r_{i}$ & Inner coil radius \\
$r_{o}$ & Outer coil radius \\
$r_{s}$ & Spindle radius \\
$r_{x}$ & Coil radius \\
$r_{y}$ & Tape spring natural radius \\
$r_{x 1}$ & Coil radius 1 \\
$R C S M$ & Relative coil sliding motion \\
$s$ & Length of a spiral \\
$T$ & Torque \\
$t$ & Thickness of tape spring \\
$T_{s}$ & Spindle torque \\
$T_{c s r}$ & Central spindle reaction torque \\
$T_{c s}$ & Central spindle torque \\
$T_{s d e q}$ & Self-deployment torque equal sense bending \\
$T_{s d o p}$ & Self-deployment torque opposite sense bending \\
$T_{s d}$ & Self-deployment torque \\
$U_{e q}$ & Strain energy stored in coiled part of tape spring in equal sense bending \\
$U_{o p}$ & Strain energy stored in coiled part of tape spring in opposite sense bending \\
$V$ & Strain energy stored in a spring \\
$v_{e}$ & Exhaust velocity \\
& Spring displacement \\
\hline
\end{tabular}




\section{Chapter 1}

\section{Background}

In recent years there has been a surge of interest in the use of large deployable sails for solar sailing missions or for use as drag deorbiting devices for satellites. These sails are stored within the spacecraft during launch and are deployed once in space. They are usually made of a thin layer of Kapton which can be coated with aluminium to increase the reflectivity of the surface for use in solar sailing applications. By reflecting sunlight, a small force is imparted to the spacecraft providing an alternate form of propulsion. While the force is very small compared to other more conventional propulsion methods, it can be used throughout the mission and does not require any fuel to be carried on board the spacecraft. This enables missions that would not otherwise be feasible. Figure 1.1 shows an artist's impression of the Japanese Aerospace Exploration Agency's IKAROS, the spacecraft that proved the concept of solar sailing (Tsuda et al., 2013, 2011).

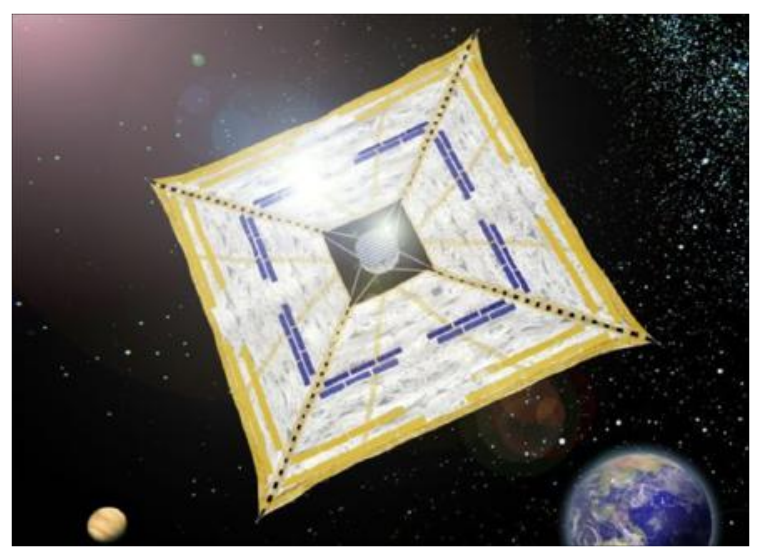

Figure 1.1: An artist's impression of IKAROS, a spin deployed solar sail developed by JAXA to prove the concept of solar sailing (JAXA, 2010).

Large sails can also be used for the drag deorbiting of satellites at the end of their missions. When a satellite is launched it will maintain its orbit for a significant period of time. By deploying a large sail, the surface area to mass ratio of the satellite will be increased, magnifying the effect of the small amounts of drag experienced in low earth orbit from the edge of the atmosphere. This causes the satellite to slow down, re-enter the Earth's atmosphere and burn up. As a greater number of satellites are launched, a method of disposing of satellites at the end of their life time is critical to minimise the amount of space debris in low Earth orbit. Figure 1.2 shows an artist's impression of NanoSail-D2 (Johnson et al., 2011; Katan, 2012). NASA designed this satellite to prove the concept of using drag sails as a deorbiting mechanism. 


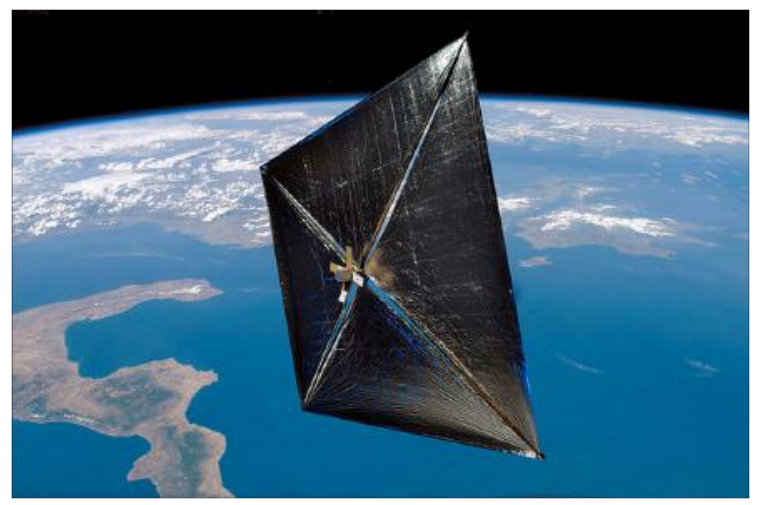

Figure 1.2: An artist's impression of NanoSail-D2. Coiled booms are used to deploy and constrain the four triangular drag sails (NASA, 2010).

NanoSail-D2 and various other missions have been developed to increase the technology readiness level of solar sailing and drag deorbiting. These include the CubeSail (Lappas et al., 2011), DeorbitSail (Stohlman et al., 2013; Stohlman and Lappas, 2013) LightSail (Biddy and Svitek, 2012; Ridenoure et al., 2015) and InflateSail missions (Viquerat et al., 2015). Unlike the spin deployed membrane of IKAROS, all of these missions use coiled deployable booms to extend and constrain their sails. The coiled booms used are similar in action to a carpenter's tape measure; they are stored in a coiled configuration inside the satellite and extend during deployment. These missions are CubeSat size satellites that use multiples of $10 \mathrm{~cm} \times 10 \mathrm{~cm} \times 10 \mathrm{~cm}$ (or ' $1 \mathrm{U}$ ') for their chassis size. Figure 1.3 shows a simplified schematic of CubeSail. The satellite is $3 \mathrm{U}$ in size with $2 \mathrm{U}$ devoted to the sail and boom deployment mechanism. Before sail deployment the chassis sides hinge along their bottom edges exposing the sails and booms. To deploy the sails, a motor turns the coiled booms. This causes the tips of the booms to exit at $90^{\circ}$ to one another, extending the sails at the same time. This is also shown in a top view in Figure 1.4. The booms continue to extend until the sails are fully deployed and tensioned as shown in Figure 1.4(c). Figure 1.5 shows the deployment process of CubeSail and the Figure 1.6 shows full deployment.

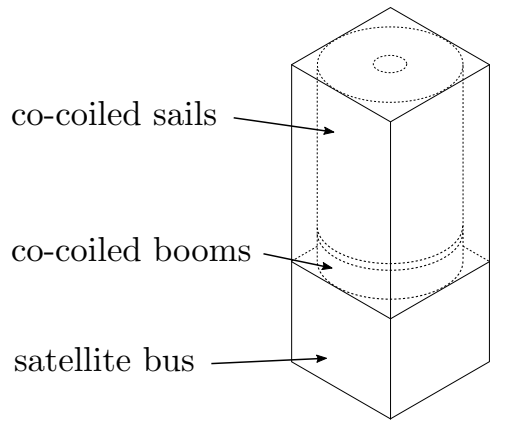

(a)

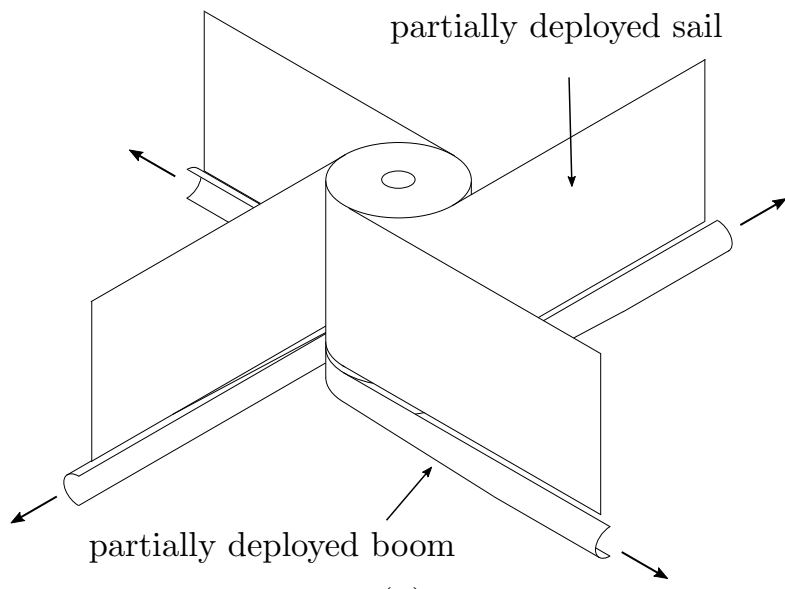

(b)

Figure 1.3: (a) CubeSail with the top section transparent to show the sails and booms. (b) The sails and booms during the initial stages of deployment. 


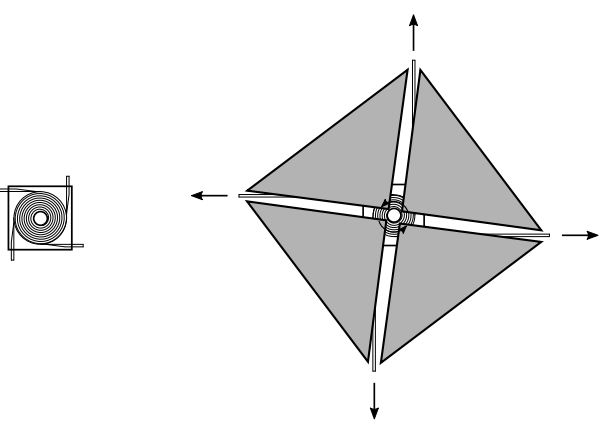

(a)

(b)

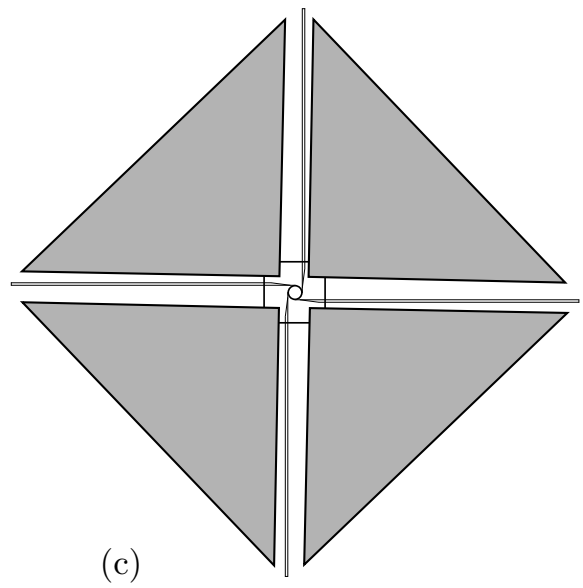

Figure 1.4: A top view of the CubeSail deployment process. (a) The booms and sails are coiled within the satellite. (b) The booms are pushed out of the deployer by the turning of the central spindle, this deployment unfurls the sails. (c) The sails are tensioned and held in place by the deployed booms.
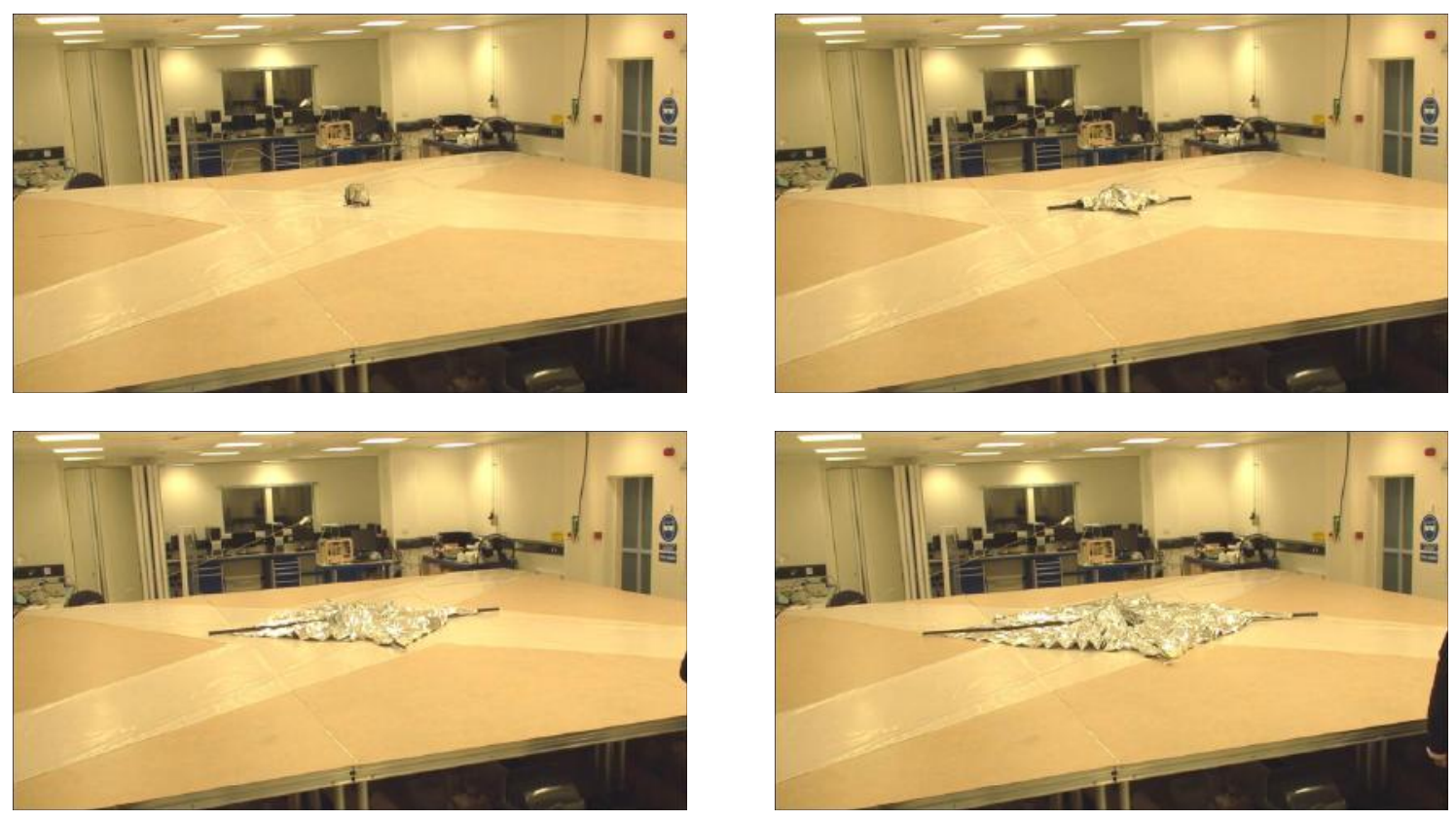

Figure 1.5: CubeSail during the deployment process. The booms extend, uncoiling and unfolding the sail. The table is approximately $6 \mathrm{~m} \times 6 \mathrm{~m}$.

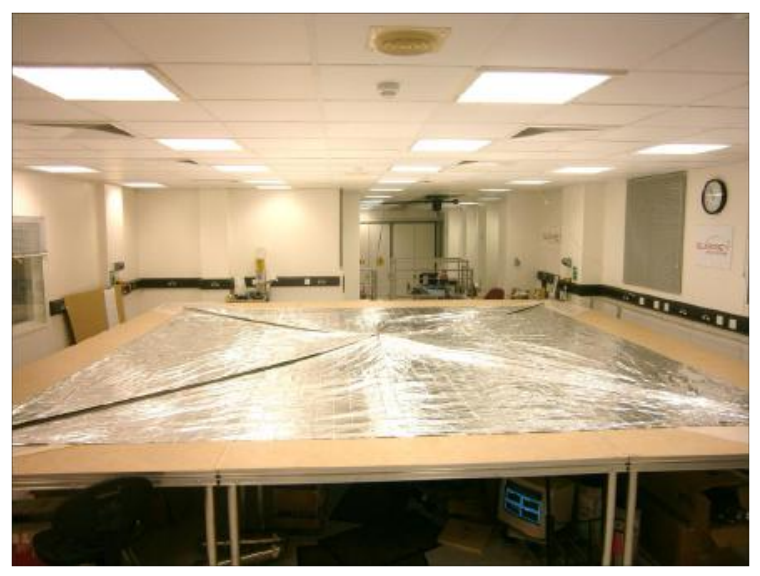

Figure 1.6: CubeSail fully deployed. The booms have deployed and tensioned the sails. 
During the testing phase of some of these missions a problem called blossoming was encountered. During normal deployment the spindle at centre of the coil of booms is turned by an electric motor and the ends of the booms extend from the spacecraft. The central spindle torque applied by the electric motor to the coiled booms is transferred to a tip force at the end of each boom. These tip forces act along the axes of the booms and are opposed by the tension of deploying the sails which causes compression within the booms as shown in Figure 1.7. If the sail tension and hence the tip force applied by extending the sail becomes too great, blossoming occurs. This is where the deployed parts of the booms and the outside of boom coil stops moving but the centre of the boom coil and motor continue to turn, unwinding the coil within the deployer as shown in Figure 1.8. This stops the deployment of the sails and jams the mechanism. The blossoming issue is not well understood and there is very little in the literature to describe its occurrence or to predict under what loads or conditions it will occur. The work carried out in this thesis aims to create an analytical frame work to describe the blossoming problem and to predict when it will occur. This will enable future boom deployer designers to use the models created during this work to inform their design process.

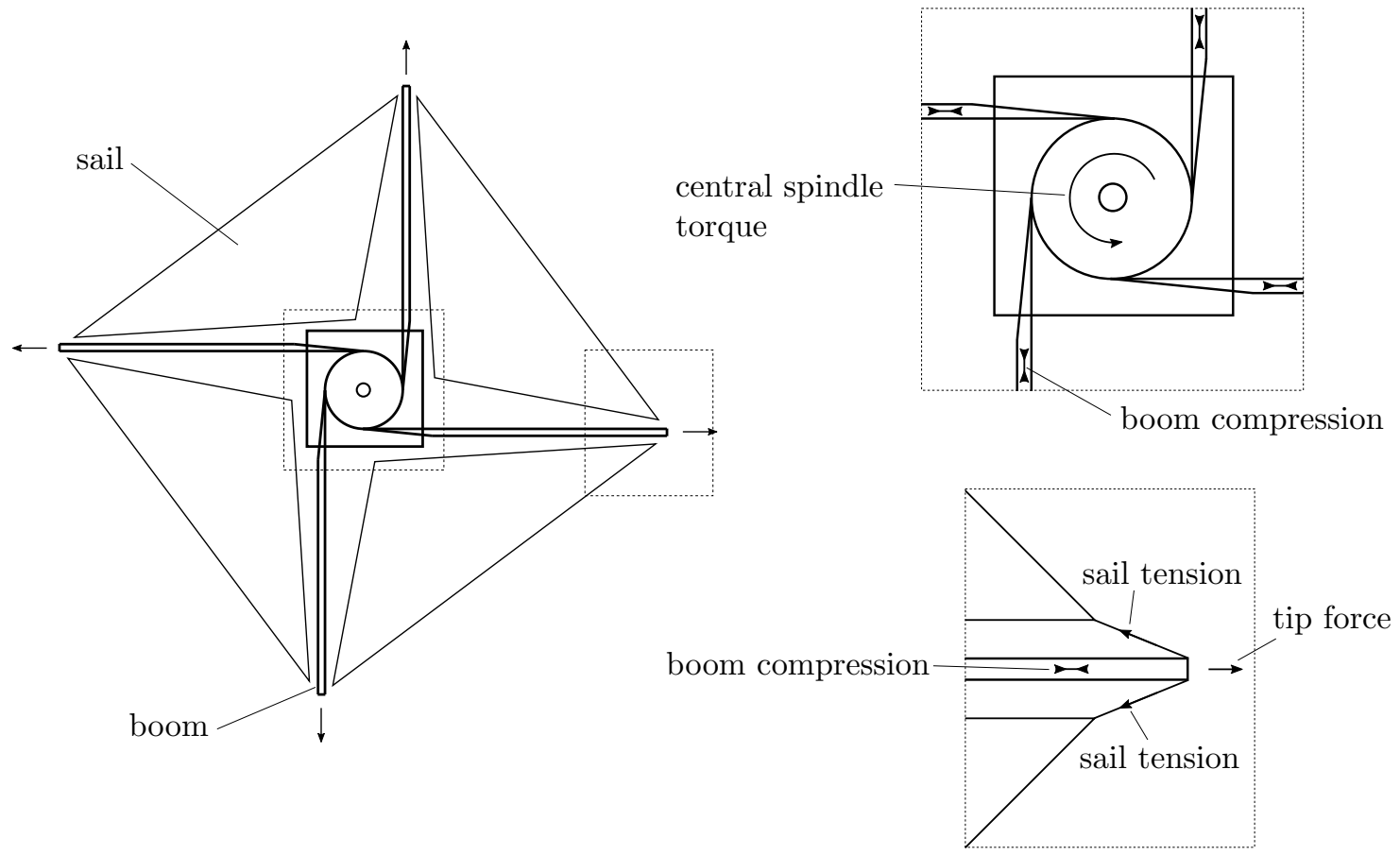

Figure 1.7: Central spindle torque, tip force and sail tension during the deployment process. 


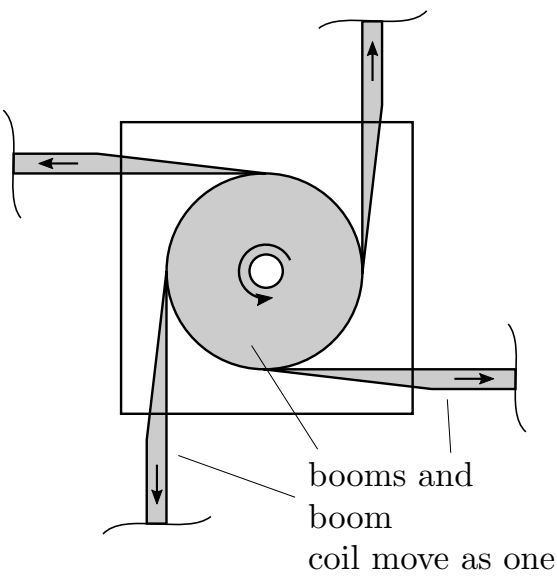

(a)

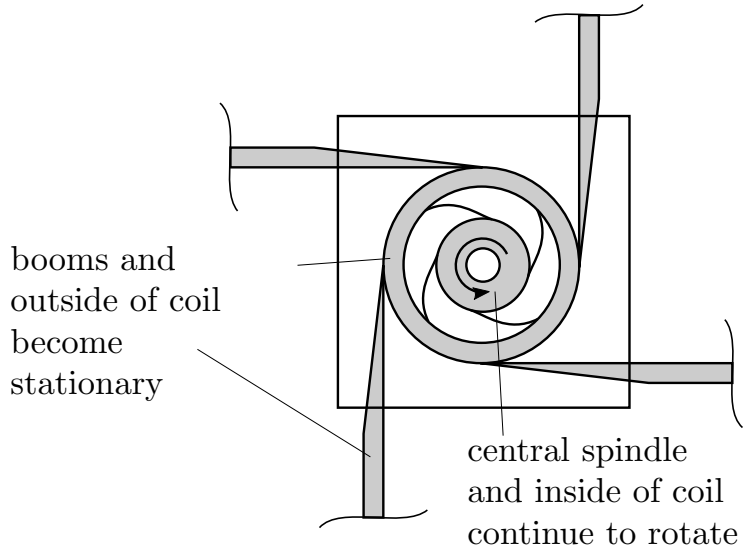

(b)

Figure 1.8: (a) Normal deployment: the booms and boom coil move as one. (b) Blossoming: the outside of the coil and the deployed booms become stationary but the central spindle continues to rotate.

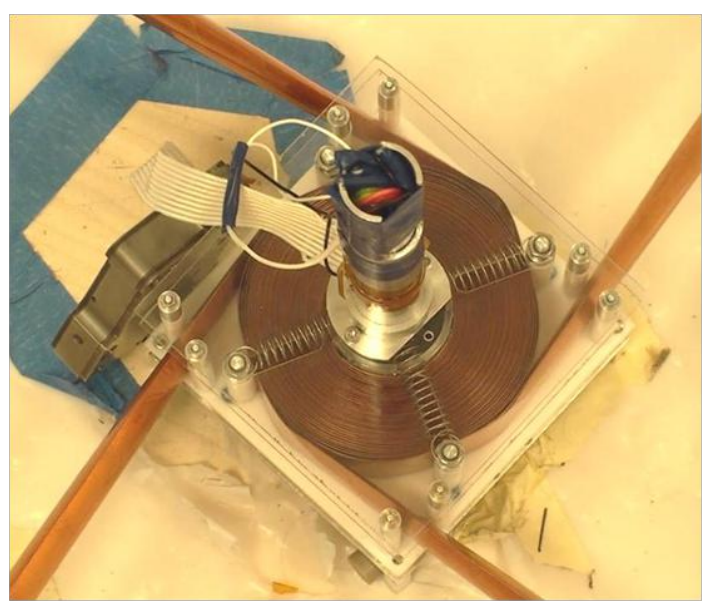

(a)

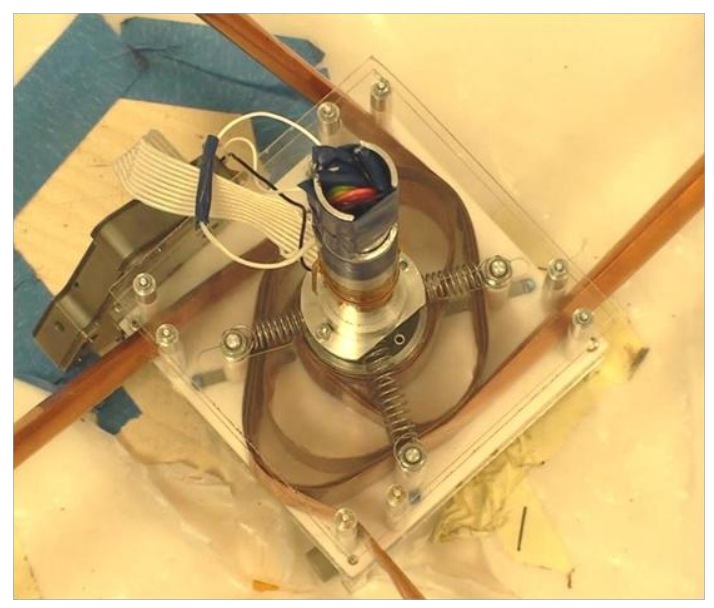

(b)

Figure 1.9: (a) The CubeSail deployer during normal deployment. (b) The CubeSail deployer after blossoming. There are large gaps between the inside and the outside of the boom coil. 


\subsection{Aims and objectives}

The aims of this thesis are to accurately describe the blossoming problem and to create a theoretical framework to describe the tip force from the boom and torque from the central spindle during blossoming for the case of a tape spring type boom. It is hoped that the methods created for tape spring type booms can be modified for other boom types and materials. Currently there is no theory to describe the tip force a coiled boom with compression rollers can exert before blossoming occurs. This leads deployer designers to rely on practical verification of their designs during deployment testing. It is hoped that this work will create a tool to predict the tip force and central spindle torque during the design phase of a project instead of having to rely on a trial and improvement method during deployment testing. To achieve this aim the following objectives were set for the work carried out during the $\mathrm{PhD}$ :

- Understand and describe the blossoming problem and the motion of the coiled boom during the blossoming process.

- Create a simple model to describe the resistance to blossoming a coiled boom exhibits on its own.

- Create a more complex model that describes the resistance to blossoming of a coiled boom including the effect that the spiral geometry of the coil and compression rollers have.

- Create a model to describe the central spindle torque during blossoming and to define the relationship between the central spindle torque and the tip force.

- Understand the relative motion between each layer of the coiled boom and the effect that the friction within the boom coil has on blossoming.

\section{$1.2 \quad$ Elements of novelty}

The elements of novelty within this work are:

- A simple model to describe the tip force of a coiled boom.

- A more complex model to describe the tip force of a coiled boom including the spiral geometry of the coil and the compression springs.

- A model of the central spindle torque and its relationship to the tip force during blossoming.

- A description of the relative motion between each layer of the coil during blossoming.

- A first approximation of the effect that friction has on the blossoming process.

\subsection{List of contributions}

During the work carried out in this thesis the following papers have been published:

- Hoskin AL. Blossoming of Coiled Deployable Booms. In 56th AIAA/ASCE/AHS/ASC Structures, Structural Dynamics, and Materials Conference 2015 (p. 0207).

- Hoskin AL, Viquerat A. An Analysis of a Coiled Tape Spring during Extension and Compression. In 3rd AIAA Spacecraft Structures Conference 2016 (p. 1470).

- Hoskin A, Viquerat A, Aglietti GS. Tip force during blossoming of coiled deployable booms. International Journal of Solids and Structures. 2017 Apr 18. 


\subsection{Outline of report}

Chapter 2 gives a literature review on the subject and introduces the problem of blossoming. Chapter 3 develops a model that uses the strain energy stored in the coiled booms and the compression rollers to find the tip force during blossoming. Chapter 4 uses the strain energy stored in the coiled booms and the compression rollers to find the self deployment torque and the central spindle torque during blossoming. Chapter 5 details the relative motion of the different layers of boom and how they slide past one another. Chapter 6 describes the design and operation of the experimental test set-up used for the experiments carried out for the thesis and Chapter 7 concludes. 


\section{Chapter 2}

\section{Introduction}

\subsection{Solar sailing and drag deorbiting}

A large majority of propulsion in space relies upon the use of reaction engines in one form or another. These create thrust by expelling propellant mass. The change in velocity $(\Delta v)$ of the spacecraft given by the use of a reaction engine is dependent on the effective exhaust velocity $\left(v_{e}\right)$ and the difference between the initial mass of the spacecraft including fuel $\left(m_{0}\right)$ compared to the mass once the fuel has been used $\left(m_{f}\right)$, as shown in the well known rocket equation (Equation 2.1).

$$
\Delta v=v_{e} \ln \frac{m_{o}}{m_{f}}
$$

This leads spacecraft designers to strive for greater and greater exhaust velocities to maximise the amount of $\Delta v$ for a given ratio of propellant to total spacecraft mass. While traditional solid and liquid fuel rocket exhaust velocities range from $1-4 \mathrm{~km} / \mathrm{s}$, more exotic propulsion systems such as hall effect thrusters, ion thrusters and pulsed plasma thrusters can have effective exhaust velocities of up to $30 \mathrm{~km} / \mathrm{s}$. The higher the exhaust velocity the greater the efficiency of the propulsion system in terms of how much $\Delta v$ and therefore how much manoeuvring a spacecraft can achieve during its mission for a given mass ratio. Alternatively, by using a higher efficiency propulsion system, the overall mass of the spacecraft can be minimised therefore reducing mission costs. Even with ever increasing efficiencies, spacecraft are always limited to a maximum $\Delta v$ by the nature of to having to carry propulsion mass on board.

Solar Sailing is a form of propulsion that uses the radiation pressure of light to propel a spacecraft. Large, thin, reflective "sails" are deployed and constrained by various means to the spacecraft. By having a large surface to reflect sunlight, a very small but constant force is applied to the spacecraft; the pressure applied to a solar sail at $1 \mathrm{AU}$ is $\approx 9 \mu \mathrm{Pa}$ (McInnes, 2004b). While this force and therefore acceleration is small, it does not require the spacecraft to have on board propellant, so can be used indefinitely for the life time of the mission. This permits a larger $\Delta v$ than can be achieved with reaction engines and also allows for continuous acceleration during the entire time the sail is deployed, enabling missions that were not previously feasible.

\subsubsection{Missions}

In 2010 the Japanese space agency proved the solar sailing concept with the IKAROS demonstrator mission. Other examples of missions that plan to use solar sailing are given below; these missions would be impossible or prohibitively expensive without solar sailing. 


\section{Geostorm}

Geostorm is a mission that would place a spacecraft in-between the Sun and the Earth, warning of an incoming solar storm (West and Derbès, 2000; Yen, 2004; West, 2004). The International Sun-Earth Explorer carried out a similar mission and investigated the Earth's magnetic field and solar winds at L1: the Earth-Sun Lagrangian point (Ogilvie et al., 1977). The concept of Geostorm is to place a spacecraft in a similar location but in an orbit that is closer to the Sun, increasing the warning time of an inbound solar storm. The solar sail will provide a constant force to offset the gravitational attraction of the Sun, enabling it to have a closer orbit.

\section{Solar Orbiter}

The Solar Orbiter mission aims to investigate the structure of the Sun at higher latitudes (Goldstein et al., 1998). Most studies into the Sun have been carried out from Earth, or from Earth orbiting satellites and therefore from near the Earth's orbital plane. The Solar Orbiter mission aims to use a solar sail to change a solar equatorial orbit of a spacecraft to polar orbit, allowing measurements of the Sun from the poles. This type of mission would be very difficult, if not impossible, without the use of a solar sail due to the large amounts of energy needed to change the inclination of the orbit.

\section{GeoSail}

GeoSail is a mission that aims to investigate and characterise the Earth's magnetotail whilst also advancing solar sailing technology (Macdonald et al., 2007; McInnes et al., 2001). The mission would put a satellite with a $43 \mathrm{~m}$ x $43 \mathrm{~m}$ square solar sail in an orbit around the Earth. Measurements of the magnetosphere would be taken both of the bow shock on the sunward side of the Earth and also of the magnetotail that trails away on the other side. Other similar missions have been proposed to investigate the magnetosphere, but by using a solar sail the satellite's orbit can be altered to keep it in a optimal position throughout the whole year as the Earth and hence the magnetotail rotates around the Sun.

\section{Solar sailing kinetic energy impactor}

Another mission investigates using a kinetic energy impactor to deflect an asteroid that is on a possible collision with the Earth. Various methods of deflection could be used including a nuclear blast, a large mirror to reflect sunlight onto the asteroid, or applying a surface coating to change the reflectivity of the asteroid to change its orbit. Alternatively by using a $330 \mathrm{~m}$ square solar sail a mass can be accelerated to a very large velocity $(60 \mathrm{~km} / \mathrm{s})$. This would impact the asteroid, deflecting it away from the Earth. A mission that uses this option would be competitive in terms of mass with the nuclear option without the politics of having nuclear weapons in space (McInnes, 2004a).

\subsubsection{Types of solar sail}

The acceleration that is achievable by a solar sail depends on the strength of the radiation pressure of light and the surface area to mass ratio of the solar sailing spacecraft. The pressure that solar radiation exerts on a solar sail at $1 \mathrm{AU}$ is $\approx 9 \mu \mathrm{Pa}$ increasing to $\approx 60 \mu \mathrm{Pa}$ at $0.39 \mathrm{AU}$, which is approximately the orbit of Mercury (McInnes, 2004b). Because this pressure is very small, the acceleration possible by a solar sailing spacecraft is also very small. To maximise the acceleration, the surface area to mass ratio of a spacecraft must be increased as much as possible. This leads to very thin reflective sails and large lightweight supporting structures. Solar sailing spacecraft must be small enough to fit within the rocket payload area and then deploy to full size once in 
space. To achieve this large surface area to mass ratio, and the ability for the spacecraft to fit inside rocket fairings then deploy once in space, there have been various different design concepts; the most common three are heligyro, spinning disc, and boom supported square solar sails.

\section{Heligyro}

A heligyro uses the centrifugal force created by spinning the spacecraft to deploy and maintain the shape of its long thin sails. These sails are attached to the spacecraft chassis and can be pitched along their radial axis to control the spacecraft's attitude and thrust in a similar way to the control of a helicopter (Blomquist, 1999). This type of solar sail can have a very high surface area to mass ratio as there is very little structure needed to support the sail material, which keeps its shape due to the centrifugal force induced by spinning. One downside of the heligyro design is its low manoeuvrability due to its long sails.

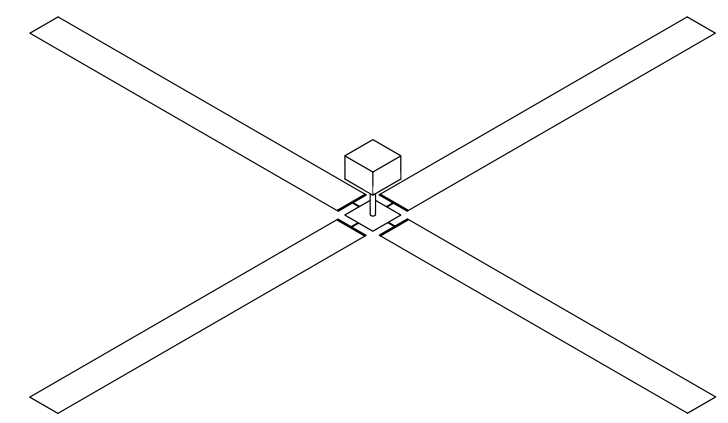

Figure 2.1: A heligyro type solar sail. The thin material sails are deployed and constrained by the centrifugal force caused by the rotation of the spacecraft.

\section{Spin deployed disc or square sail}

A spin deployed disc or square solar sail employs a similar principle to the heligyro in that it uses the centrifugal force of spinning to deploy and give shape to the sail material. Instead of having long thin blades, the sail is a disc or square shape. The Japanese Space agency launched IKAROS in 2010; this was the first demonstration of solar sailing in space (Tsuda et al., 2011, 2013). It used a spin deployed square sail with corner weights to aid deployment. Another mission that used a spin deployed sail was the Znamya 2. The concept was to use the sails as a mirror to illuminate northern parts of the Earth. The $20 \mathrm{~m}$ diameter sail consisted of petals of $5 \mu \mathrm{m}$ thick Mylar film which were deployed and tensioned by rotating the whole structure (Garner et al., 1999; Gärdsback and Tibert, 2009).
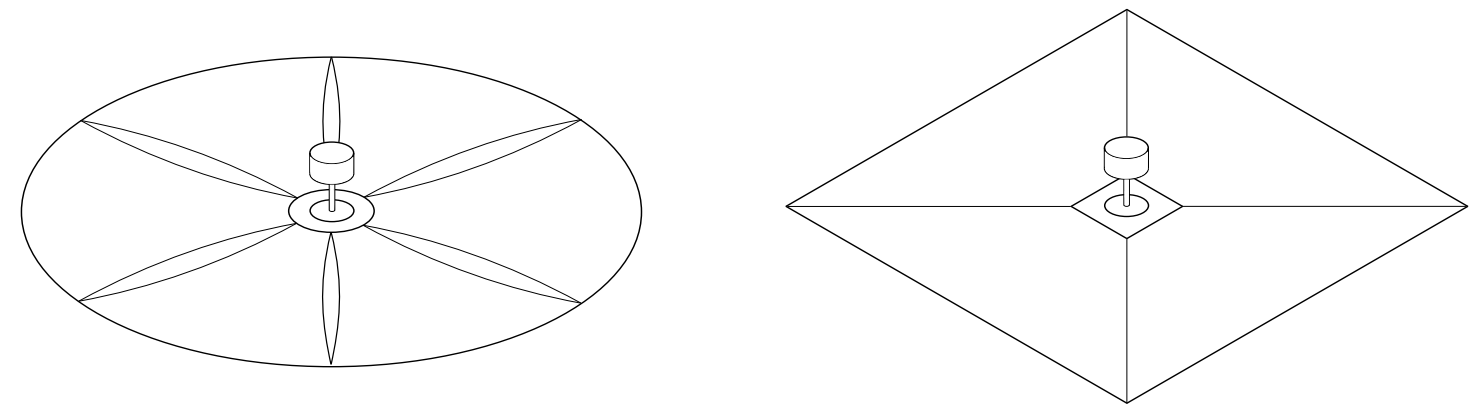

Figure 2.2: Circular and square spin deployed solar sails. Both sails types are deployed and constrained by the rotation of the spacecraft. 


\section{Boom deployed square sail}

A square solar sail has four triangular sails that are deployed and constrained by four deployable booms. These four triangles form a square shape. The advantage of the square solar sail over other designs is that is does not have to spin to maintain its shape. This makes deployment and control simpler, and allows for more rapid attitude changes. Deployment testing on the ground is also less complicated with a boom deployed sail as the effect of the atmosphere can be neglected. For a spin deployed sail, ground testing poses a challenge as both atmospheric and gravitational effects can cause the sail to behave differently compared to deployment in space. A majority of the initial technology demonstrator missions seem to prefer the boom deployed square sail due to its relative ease of ground testing and more reliable deployment (McInnes, 2004b).

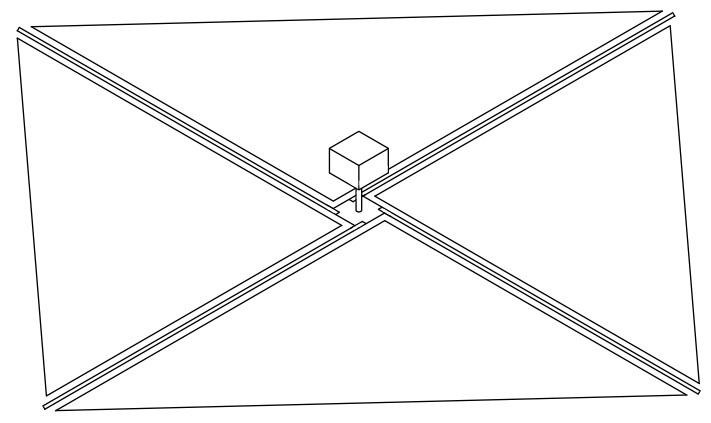

Figure 2.3: Square solar sail. The sail is deployed and constrained by four deployable booms.

\subsubsection{Current demonstrator missions}

While IKAROS can be considered a fully functional solar sailing mission there are a variety of other demonstrator missions that aim to increase the technology readiness level of solar sailing or drag deorbiting.

\section{IKAROS}

IKAROS was the first mission to prove the concept of solar sailing and was developed by JAXA the Japanese Aerospace Exploration Agency. It was launched in 2010 into an Earth-Venus trajectory. The spacecraft weighed $307 \mathrm{~kg}$ and had a $16 \mathrm{~kg}$ square sail. The sail was spin deployed and tensioned, had a surface area of $20 \mathrm{~m}^{2}$ and was $7.5 \mu \mathrm{m}$ thick (Tsuda et al., 2011). It used solar cells mounted on the sail as a power source and also used a reflectance control device to make parts of the sail more reflective than others as a steering mechanism (Tsuda et al., 2013).

\section{NanoSail-D2}

NanoSail-D was built by NASA to validate the deployment of a solar sail in space and to prove the concept of using a large deployable structure as a deorbiting device (Johnson et al., 2011; Katan, 2012; Alhorn et al., 2011). The satellite used a 3U chassis size which deployed a square sail with a side length of $3.16 \mathrm{~m}$ giving an area of $10 \mathrm{~m}^{2}$. To deploy and constrain the square sail which consisted of four triangular quadrants, four coiled booms were used. The booms were the Airforce Research Laboratory's TRAC boom (Banik and Murphey, 2010). The original NanoSail-D failed to get to orbit after a problem with Falcon-1 launch vehicle. NanoSail-D2 was a ground spare for the original mission and was launched in November 2010. The mission was a success with the satellite deorbiting rapidly once the sail was deployed. 


\section{DeorbitSail}

The DeorbitSail mission was to prove the concept of rapid deorbiting using atmospheric drag (Stohlman and Lappas, 2013; Fernandez et al., 2013; Stohlman et al., 2013). It used a platform similar to that of NanoSail-D with a $3 \mathrm{U}$ chassis deploying a $5 \mathrm{~m}$ x $5 \mathrm{~m}$ square solar sail. DeorbitSail used a variant of DLR's lenticular, closed section boom (Sickinger and Herbeck, 2002). DeorbitSail also used a translation stage to allow the centre of gravity of the satellite bus to be offset from the centre of pressure of the sail. This is used as a steering mechanism and can produce a pitch and yaw moment.

\section{LightSail}

LightSail was a project developed by The Planetary Society to advance solar sailing and to demonstrate the solar sail deployment process. (Biddy and Svitek, 2012; Ridenoure et al., 2015). It used a $3 \mathrm{U}$ platform to deploy a $5.6 \mathrm{~m} \times 5.6 \mathrm{~m}$ square solar sail giving a surface area of $32 \mathrm{~m}^{2}$. It used four TRAC booms from the Airforce Research Laboratory to deploy and constrain the sail. Although the mission experienced some communication issues, the sail eventually deployed and successfully deorbited the satellite.

\section{InflateSail}

The InflateSail project aimed to develop the use of inflatable deployable structures in space. The concept was to couple a square drag sail, deployed and tensioned with four booms, with an inflatable mast to extend the sail away from the body of the satellite (Viquerat et al., 2014). This offset distance created by the inflatable mast would give a natural stability to the satellite and sail during deorbiting due to the distance between the centre of pressure of the sail and the centre of mass of the satellite. The drag sail had a $10 \mathrm{~m}^{2}$ area and was deployed and constrained by four bistable carbon fibre booms developed at the Surrey Space Centre (Fernandez et al., 2014).

\subsubsection{Drag deorbiting}

With ever increasing numbers of satellites being launched into orbit, space debris is a growing concern. Once satellites are launched, they do not require any form of power to maintain their orbit, apart from small station keeping adjustments. This is useful during their serviceable lifetime, but once their missions are finished they are usually abandoned and stay in orbit almost indefinitely without any form of control. If two out of control satellites collide, they create a huge number of smaller debris possibly causing more collisions. This could set off a chain reaction of collisions rendering low earth orbit almost impossible to use. This effect is called the Kesseler syndrome (Kessler and Cour-Palais, 1978). Large deployable sails can be deployed by satellites in low Earth orbit at the end of their lifetimes to act as a deorbiting aid. By increasing the surface area to mass ratio of the satellite, the small amount of drag from the edge of the atmosphere can be used to slow a satellite enough for it to deorbit. The idea of mandatory deorbiting satellites at the end of their lifetimes is currently being investigated and could soon be implemented on all future satellites (E Roberts and Harkness, 2007).

\subsection{Coiled deployable booms}

The origins of coiled deployable booms can be traced back to the humble carpenter's measure. The original coiled tape measure was patented by A. J. Fellows in 1868 (Fellows, 1868). This invention consisted of a case, a flat cross section measuring tape, and a spiral spring which provided a tension to recoil the tape, as shown in Figure 2.4. A later version, which resembles the modern day tape 
measure, was patented by Hiram A. Farrand in 1922 (Farrand, 1922). This device differed to Fellows' in that the measuring tape had a curved cross section in its deployed state, as shown in Figure 2.6. This increased its stiffness and gave it a rod like form once deployed, making it more convenient to measure objects.

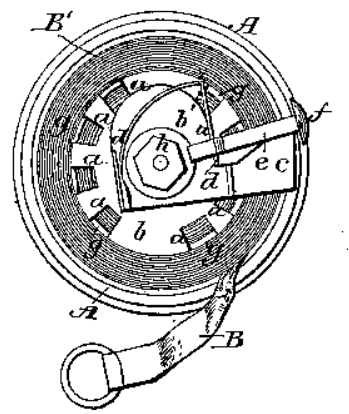

Figure 2.4: The original tape measure concept by A.J.Fellows with a central spiral spring to aid the recoiling of the measuring strip (Fellows, 1868).

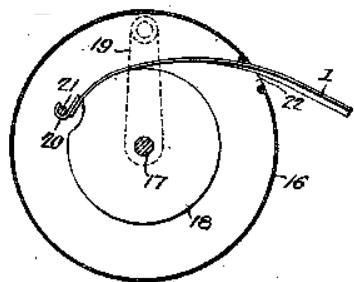

Figure 2.5: The tape measure with a curved cross section patented by Farrand (Farrand, 1922).

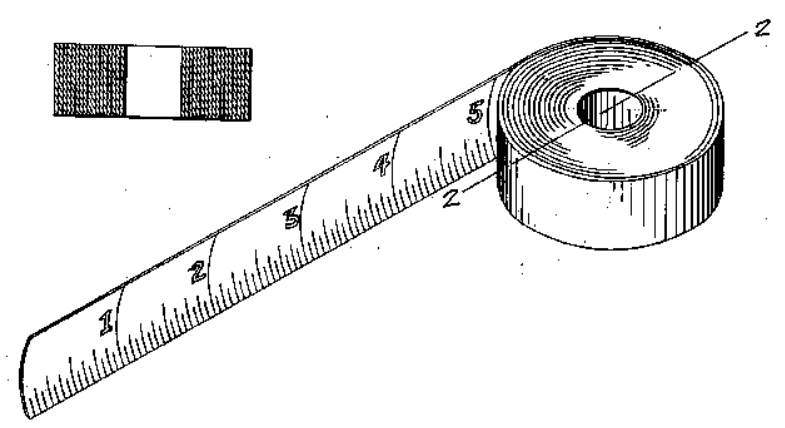

Figure 2.6: The tape measure in its two states (Farrand, 1931). When coiled the tape measure has a flat cross section but when it is extended it has a curved cross section, improving its stiffness.

The next notable milestone in the development of coiled deployable booms was the work carried out by the inventor George Klein at the National Research Council of Canada (Bourgeois-Doyle, 2004). George Klein worked with Harry Stevinson to develop a radio beacon to be dropped from military aircraft over enemy territory or hazardous terrain, to aid with navigation. The design consisted of a casing that would be dropped via parachute. Once on the ground, a self righting mechanism would operate and a deployable boom would extend to act as an antenna. This initial design would be the founding idea for the STEM booms used on the Allouette satellite (Mar and Garrett, 1969) and many other missions. The concept of the STEM boom was developed into many different forms including bi-stem, where two booms were unfurled inside one another, interlocked, where the two edges of the boom would join and lock together once deployed, and welded seam mast, where two omega shaped halves were welded together to form a lenticular shape (Herzl, 1970). STEM booms were used for a variety of different applications, hence work was carried out to investigate a number of characteristics including the bending stiffness, torsional stiffness and deployment mechanics (Rimrott, 1965a,b; Abercrombie, 1971; Elliott and Rimrott, 1966; Rimrott, $1967,1980)$. Some work focused on the use of shallow angle tape spring type booms as hinge 
mechanisms for deployable panels (Walker and Aglietti, 2007b,a; Seffen and Pellegrino, 1997). Later developments included work on different cross section booms, such as the Triangular Rollable And Collapsible boom (TRAC) and DLR's lenticular boom, as shown in Figure 2.7. The lenticular boom has a significantly improved torsional stiffness compared to other boom types as it has a closed cross section (Sickinger and Herbeck, 2002). The TRAC boom was developed to improve the second moment of area to packaged height ratio compared to other boom cross sections (Banik and Murphey, 2010).

The use of fibre reinforced composites increased the stiffness and strength properties of booms as well as allowing the development of bistability. An invention by Andrew Daton-Lovett used fibre reinforced composites to create a boom that has two stable states. With careful tailoring of the composite layup, a boom can have a second local strain energy minimum when in the coiled configuration. This created a boom that didn't require constraints to be maintained in a coiled configuration, which is useful for a number of applications such as deployable radio masts, deployable tripods and deployable ladders (Iqbal and Pellegrino, 2000; Guest and Pellegrino, 2006; Fernandez et al., 2014; Galletly and Guest, 2004a,b).

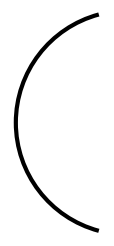

(a)

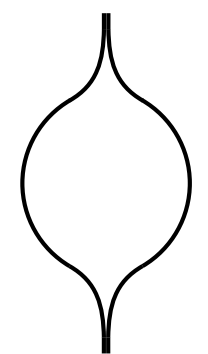

(b)

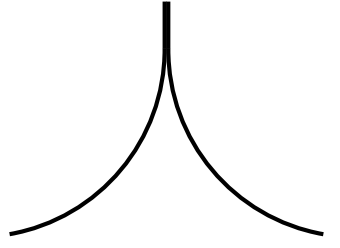

(c)

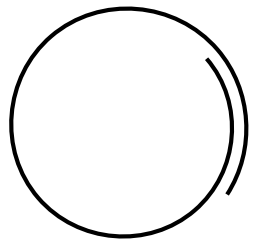

(d)

Figure 2.7: A selection of boom cross sections. (a) Tape spring. (b) Lenticular. (c) Triangular Rollable and Collapsible (TRAC). (d) Storable Tubular Extendible Member (STEM).

The various boom types all follow the same concept as a tape measure; the cross section of the boom is flat when coiled around a spindle and then changes cross section shape when deployed. Figure 2.8 shows a tape spring boom transitioning from the coiled to deployed state. (a) is the unstressed, deployed state and (b) is the transition or "ploy" region where the boom changes from a curved to a flat cross section. The boom then forms a curvature in the longitudinal direction to create a coil.

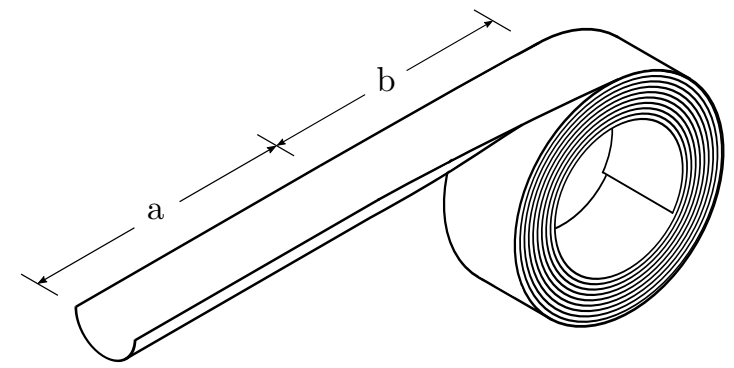

Figure 2.8: A partially coiled tape spring boom. (a) is the unstressed, deployed state. (b) is the transition or ploy region where the cross section changes from curved to flat. The boom then forms a coil with no lateral curvature but with a curvature in the longitudinal direction.

Coiled booms are usually stored in a deployer until they are ready for use. This is a device that 
constrains the boom and aids its deployment, and in some cases retraction, at the appropriate time. The three main deployer types are shown in Figures 2.9 and 2.10. All the deployers have the boom coiled around a central spindle in a stored state. The end of the boom leaves the deployer and changes into its unstressed state with a curved cross section.

The pusher deployer has a motor attached to the central spindle. To deploy, the central spindle and the coiled boom are turned, the coiled boom on the outside of the coil is pushed through the guide rollers and out of the deployer.

The puller type deployer has two or more powered guide rollers that rotate to extend the boom. Usually the central spindle has some kind of friction brake to offer a small amount of resistance to the deployment so as to stop the boom from uncoiling in the deployer.

The co-coiled deloyer has a main boom that is coiled with another strip of flexible material. To deploy, the thin strip of material is pulled and coiled onto a second spindle, deploying the main boom. As with the puller type deployer the central spindle usually has some kind of friction brake.

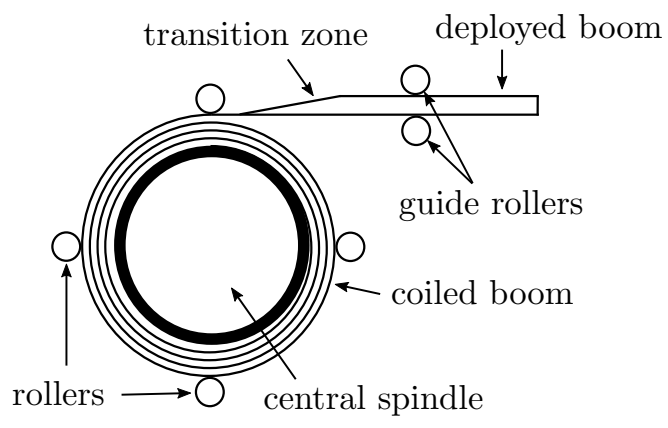

(a) Parts of a deployer.

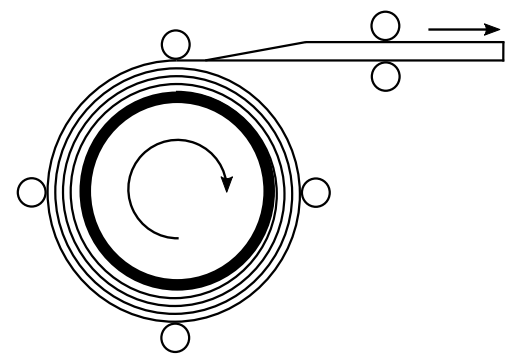

(b) Pusher deployer.

Figure 2.9: The pusher type deployer turns the central spindle to deploy the boom. Rollers stop the boom coil from unwinding and guide rollers maintain the direction of the boom tip.

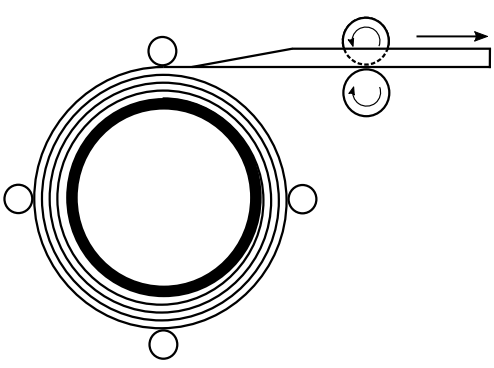

(a) Puller deployer.

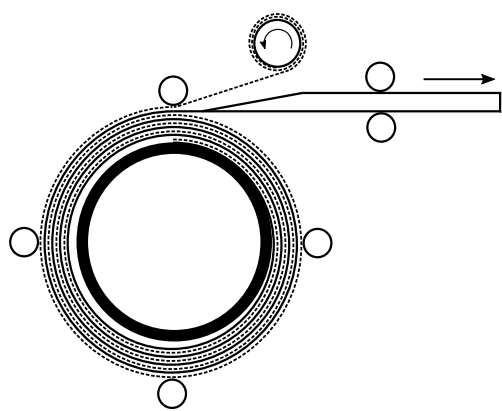

(b) Co-coiled deployer.

Figure 2.10: The puller type deployer uses two motorized guide rollers to pull the boom out of the deployer. The co-coiled deployer has a second strip that is coiled with the main boom. A separate roller pulls the second strip, deploying the main boom.

A majority of the original STEM booms used the puller or the co-coiled type deployers for single booms. This gave high reliability and in some cases the ability to retract and extend the boom multiple times. The latest solar sailing demonstration missions such as LightSail, NanoSail-D2, CubeSail and InflateSail use the pusher type deployer (Johnson et al., 2011; Biddy and Svitek, 2012; Viquerat et al., 2015; Lappas et al., 2011). These missions use the CubeSat format with a volume of multiples of $10 \mathrm{~cm}$ x $10 \mathrm{~cm} \mathrm{x} 10 \mathrm{~cm}$ for the satellite chassis, so space is at a premium. 
The pusher deployer type allows for a design that is simple and more space efficient compared to the puller or co-coiled methods. These missions also have four booms that are co-coiled around a central spindle, each boom then exits the deployer at $90^{\circ}$ to one another. Creating a four boom puller or co-coiled deployer would be more complex and heavier compared to the pusher type. One apparent downside to the pusher type deployer, which has been reported in some of the solar sailing missions and in earlier missions using pusher type deployers is blossoming (Herzl, 1970; Fernandez et al., 2013; Stohlman et al., 2013). Blossoming is an issue that occurs during deployment where the booms uncoil inside the deployer instead of deploying.

\subsection{CubeSail deployment process}

\subsubsection{Sail forces}

During the deployment of a four boom square solar sail there are two distinct phases. The first phase is the extension of both the sails and booms. In most designs the boom tips pull the two sail corners that are attached to each boom. The second phase is the final tensioning of the sail. There are various methods of achieving the correct amount of tension within the sail. One method is to have a linear spring or a spring with a low stiffness and fairly long extension length between the boom tip and the corners of the sail. The booms can then be extended to a pre-set length and the sail will obtain a certain tension in each corner. The use of a spring between the boom tip and the corners of the sail make the precise length of boom deployment less critical. It also allows for any difference in expansion and contraction between the booms and sails during the large range of temperatures that will be experienced in space.

Finding the force needed to deploy a sail during the first phase is a non-trivial matter and is heavily dependant on each sail and deployment mechanism. Some of the factors effecting the amount of force needed to deploy a sail include: the material thickness, the material stiffness and any static attraction between different layers of the sail. The mechanism that constrains the sails, the folding pattern, depth of the folds and the shape of the sail will also effect the force needed to deploy the sail. Due to the large number of differences apparent between each mission the best way to find the forces needed to deploy a certain sail are to carry out practical tests. The final tensioning of the sail is a more controllable process and work has been carried out to find a suitable tension force for given sail parameters. The final tensioning of the sail will also depend on the sail material, thickness and the shape of the sail. An estimate of the force needed to suspend sails is given by Greschik (Greschik et al., 2003). Other work has been carried out to determine the effect that the tension applied to the corners has on the wrinkling patterns of the sail (Su et al., 2003; Blandino et al., 2002).

\subsubsection{CubeSail deployment}

The CubeSail project used a pusher type deployer with four booms to deploy four triangular sails, as shown in Figures 2.12 and 2.13. The sails are z-folded and then coiled around a spindle which is in a separate compartment within the deployer. The packaging process of $z$-folding and coiling the sails is shown in Figure 2.11. Figure 2.11(a) shows one sail quadrant with fold lines. (b) shows the z-folding process of one sail quadrant. (c) shows the connection of the four sails to the central spindle. (d) shows the central spindle being turned to co-coil the four sails. During deployment the booms extend from the deployer, pulling the corners of the sail quadrants, reversing the process shown in Figure 2.11. Figure 2.12 shows the deployment sequence of the sails and the booms. 


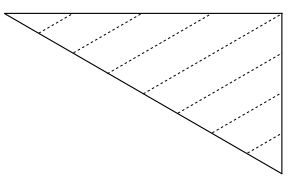

(a)

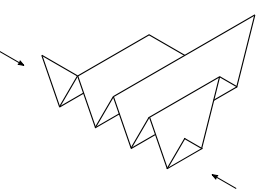

(b)

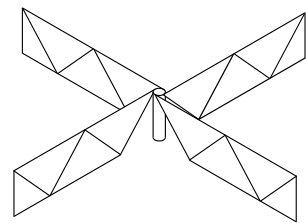

(c)

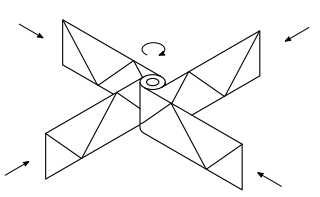

(d)

Figure 2.11: The z-folding and coiling of the sails. The sails are $z$-folded, the $90^{\circ}$ corner is attached to the spindle. The spindle is then turned to coil the sails.

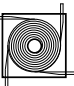

(a)

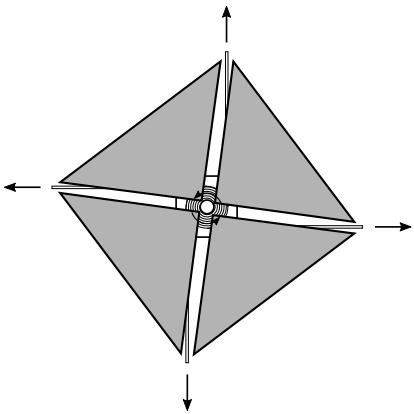

(b)

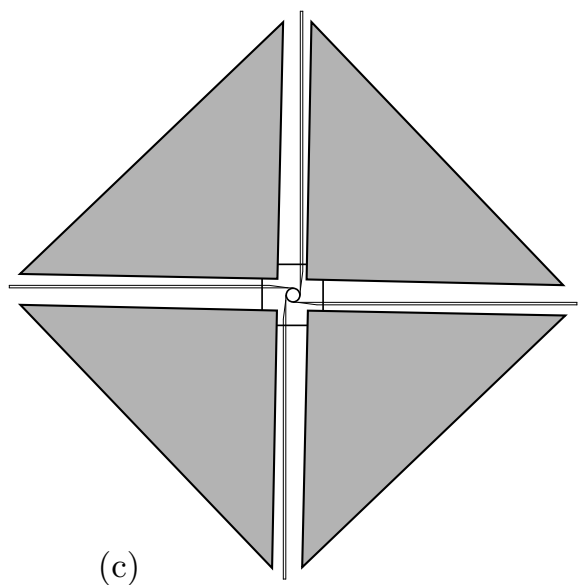

(c)

Figure 2.12: The deployment sequence of CubeSail. (a) The booms and sail are initially coiled and folded within the deployer. (b) The booms extend and pull the tips of the sails uncoiling and unfolding the sail. (c) The booms are fully extended and the sails are tensioned.

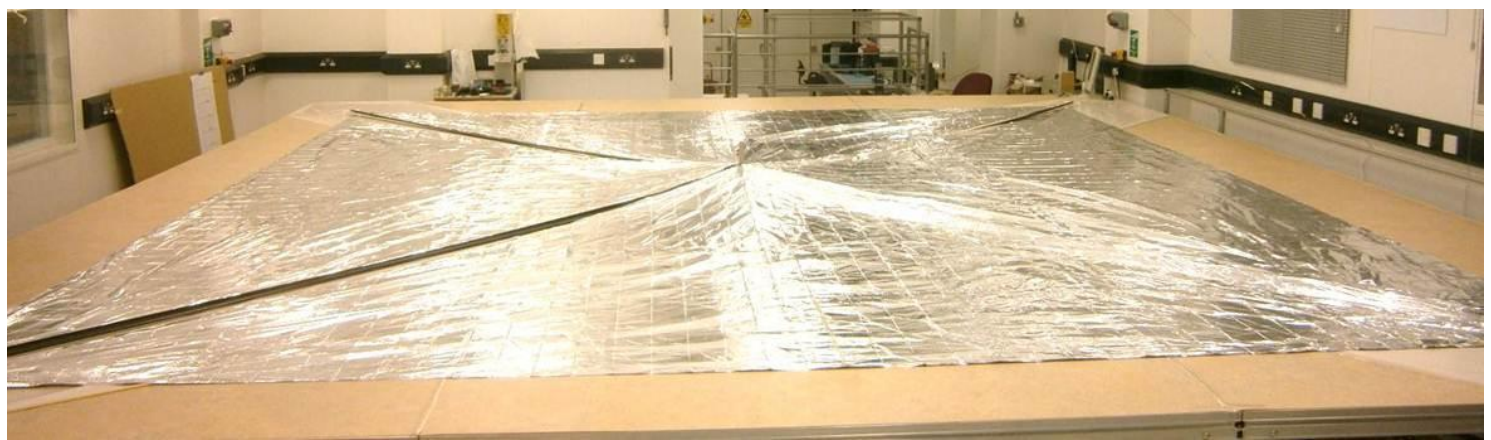

Figure 2.13: A picture of CubeSail in a fully deployed state during testing. The booms have extended and tensioned the sails. The outside edges of the sail are $5 \mathrm{~m}$ long.

The CubeSail deployer has booms that are co-coiled. This is where two or more booms are coiled together around a single spindle. The roots of the booms are usually attached to the spindle at an angle that correlates to the number of booms, i.e. two booms would be attached at $180^{\circ}$ to one another and four booms at $90^{\circ}$. Figure 2.14 shows two co-coiled booms attached to the central spindle at $180^{\circ}$ to one another. 


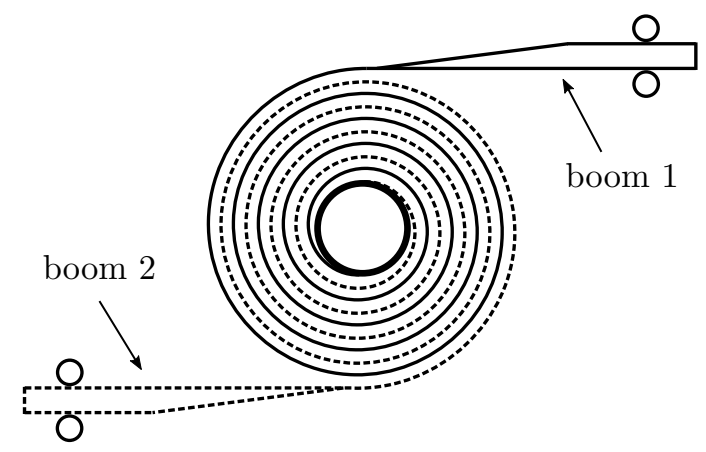

Figure 2.14: Two co-coiled booms. The roots of the boom are connected to the central spindle at $180^{\circ}$ to the other one. As the central spindle turns one boom is layered on top of the other.

Figure 2.15 shows four co-coiled booms in the configuration that is used in the CubeSail deployer. When the booms are coiled they have a flat cross section, as they leave the boom coil they transition to a curved cross section.

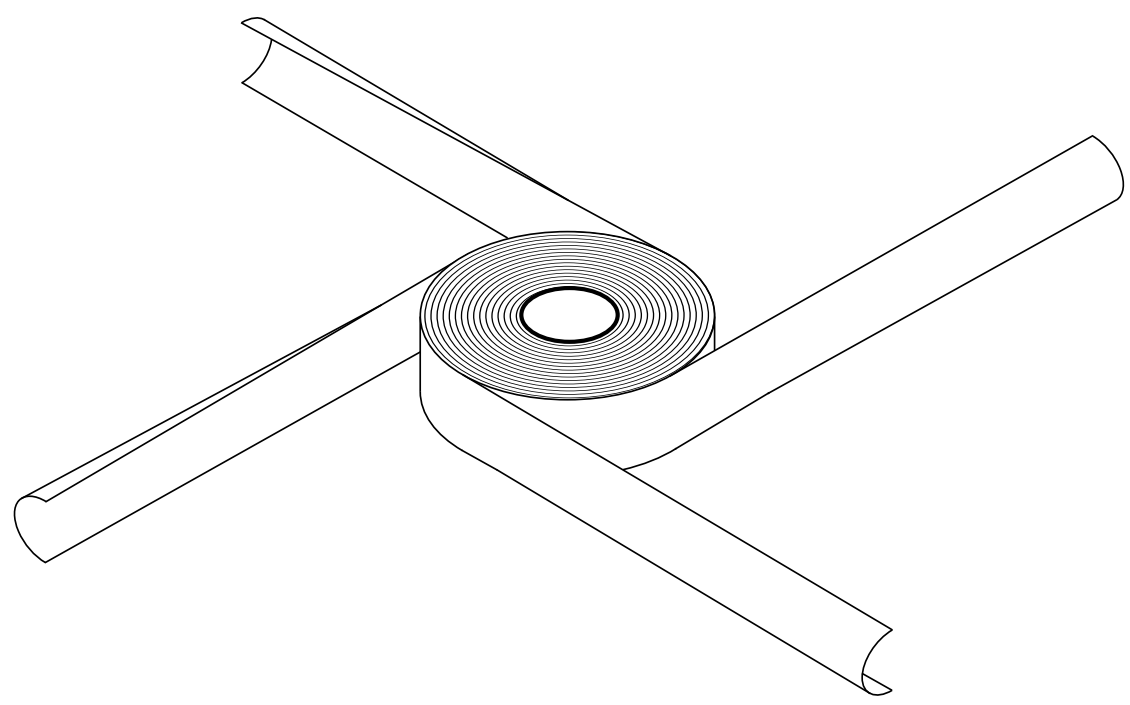

Figure 2.15: Four co-coiled booms. The boom cross section is flat when it is in the coil, there is then a transition zone where the boom cross section changes from flat to curved.

Figure 2.16 shows the workings of a deployer. The four booms are co-coiled around a central spindle. The ends of the four booms exit the deployer at $90^{\circ}$ to one another, changing from a flat cross section to a curved one through the transition zone. The guide rollers aid the deployment and constrain the angle that the booms leave the deployer. The compression rollers compress the boom coil to aid the packing of the deployer and to stop the boom coil from expanding. During deployment a motor rotates the central spindle which turns the coiled booms, the deployed parts of the booms move away from the deployer extending the sail. As the booms are deployed, the boom coil decreases in diameter. The compression springs pull the compression rollers against the coiled booms, stopping them from expanding. 


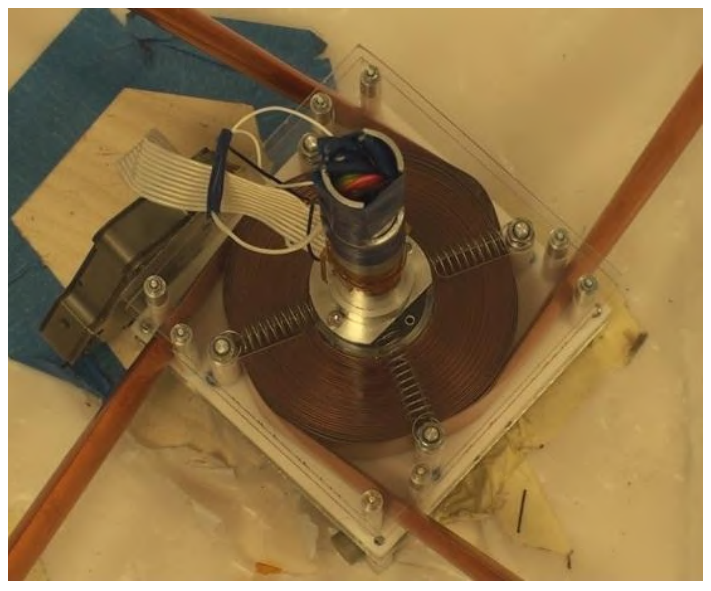

(a) The CubeSail deployer.

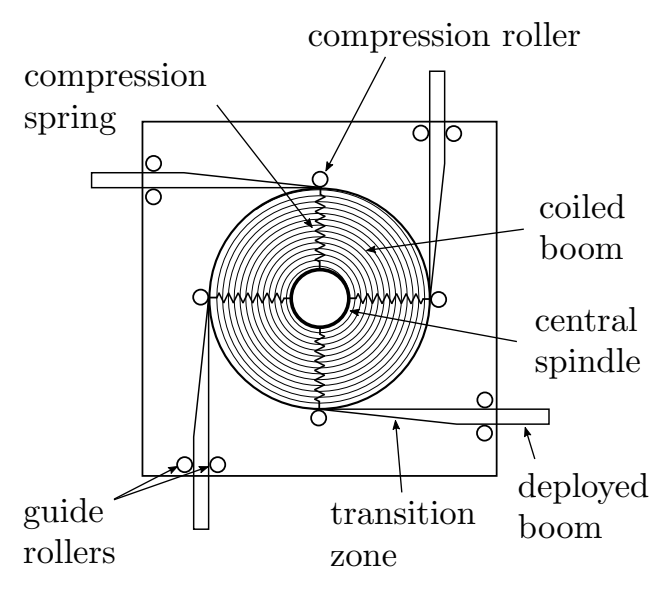

(b) Schematic of the CubeSail deployer.

Figure 2.16: An image and a diagram of the CubeSail deployer before deployment.

Figure 2.17 shows the torques and force that a boom creates and applies to its surroundings when it is coiled. Figure 2.18 shows the reaction torques and forces that need to be applied to the coiled boom to stop it uncoiling or deploying. The coiled boom has a tendency to uncoil by itself. Where the boom leaves the boom coil and changes cross section, it applies a self deployment torque $\left(T_{s d}\right)$. This tries to move the deployed part of the boom in an anti-clockwise direction. This torque is opposed by a force from the guide roller. The other end of the boom is attached to the central spindle. The coiled boom would obtain a lower strain energy state if it could unwind, this leads to a central spindle torque $\left(T_{c s}\right)$. In the CubeSail deployer the central spindle rotation is controlled by a motor, this provides a torque to oppose the central spindle torque applied by the boom, this is termed the central spindle reaction torque $\left(T_{c s r}\right)$. The compression rollers also apply a force to the outside of the boom coil to help to aid the winding in of the boom and to stop the boom coil expanding during deployment.

To uncoil and tension the sail, the boom applies a tip force to the sail corners. The tip force arises from overcoming the sail tension and is equal and opposite to it. If there are no sails attached, there will be no tip force. The magnitude of the sail tension and hence tip force will vary during the deployment process and depends on a variety of factors.

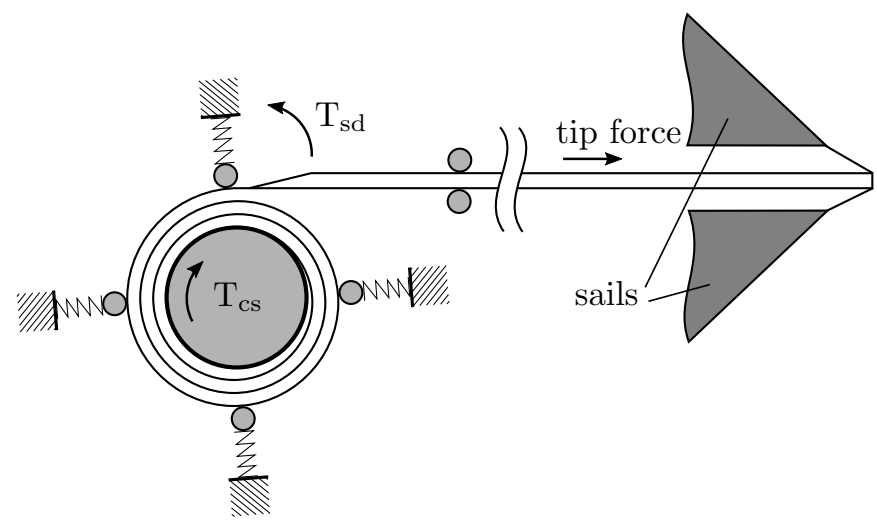

Figure 2.17: The force and torques applied by the coiled boom. (Note: the compression roller springs in this diagram are compression springs as opposed to tension springs which are used in the CubeSail deployer, as shown in Figure 2.16. This change is only for the clarity of the diagram and the force in both cases is towards the central spindle.) 


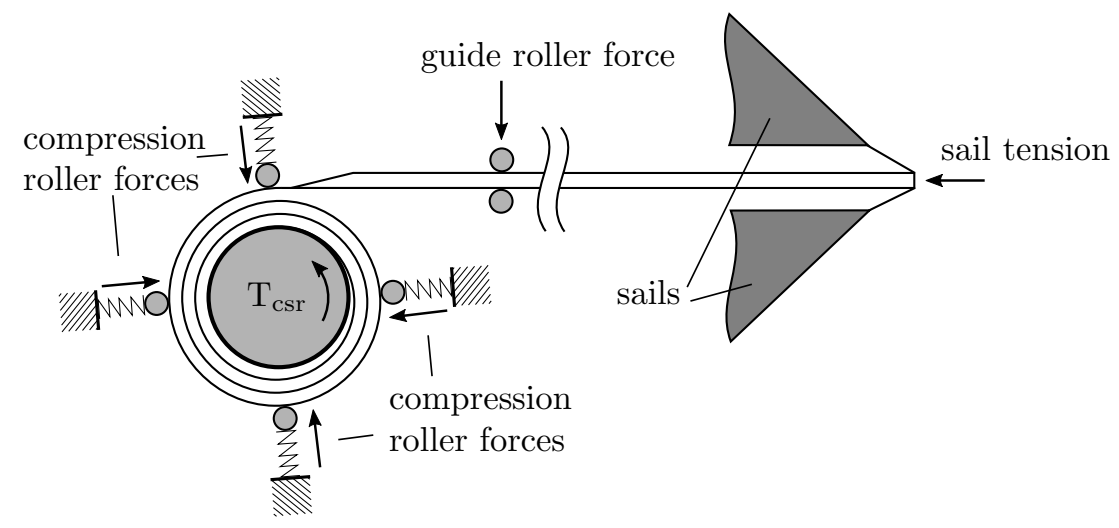

Figure 2.18: The reaction forces and torque applied to the coiled boom. As the boom deploys it extends the sails. The sails will resist deployment due to friction and the unfolding process, this gives rise to the sail tension.

There is a maximum tip force that can be applied by the sail before the deployed boom will undergo Euler buckling. To a first approximation a boom can be thought of as a column with a fixed boundary condition at the connection between the deployer end and a free condition at the boom tip where the two sail quadrants attach. The value of this critical tip force is given by Equation 2.2 where $E$ is the Young's modulus of the material, $L$ is the length of the boom and $I$ is the second moment of area of the boom cross section (Young and Budynas, 2002). This sets an upper limit of how much tip force and hence sail tension a boom can create before failing due to buckling.

$$
P_{c r}=\frac{\pi^{2} E I}{4 L^{2}}
$$

\subsubsection{Blossoming}

During the testing of the CubeSail deployer without any sails attached, the booms deployed as they were designed to do as shown in Figure 2.19. The central spindle would turn, the boom on the outside of the coil would move out of the deployer and change cross section into the deployed boom shape. As the boom coil decreased in radius the compression springs would move towards the central spindle, keeping a force on the outside of the coil. When the sails were added, the booms had to overcome the force of tensioning the sail. This sometimes caused blossoming to occur, as is shown in Figure 2.20. In this case instead of the coiled boom turning as one and transferring the torque from the central spindle to the outside of the boom coil and to the deploying booms, the boom on the outside of the coil stopped moving, the coiled boom layers slid past one another, uncoiled and grew inside the deployer, stopping the deployment. As the coil grew within the deployer, the compression springs were compressed and gaps grew between the central spindle and the coiled boom. The deployment of the booms stopped, and if the central spindle rotation continued, the roots of the booms would be damaged at their attachment points to the central spindle. The blossoming effect was not consistent. It did not occur on every deployment and when it did, it did not always occur at the same point in the deployment process. This may have been due to a slightly different sail tension due to hand packing of the sail. It is thought that the deployer could produce a certain tip force without blossoming but above this tip force blossoming 
would occur.
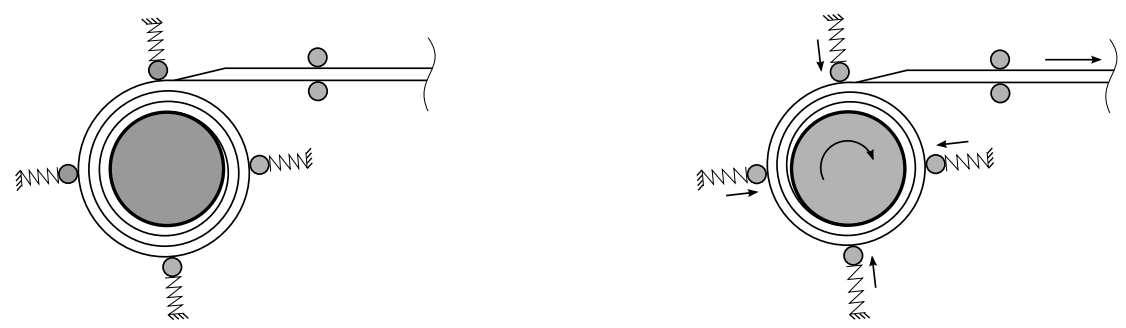

Figure 2.19: Normal deployment. The central spindle and coiled boom turn as one and the part of the boom on the outside of the coil leaves the deployer.
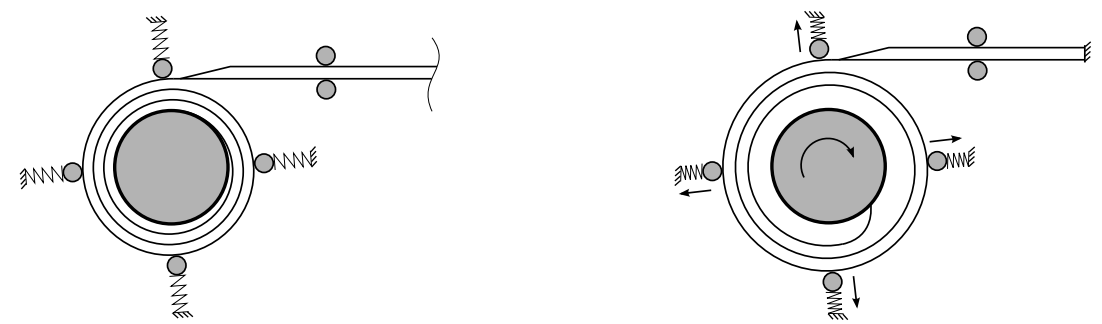

Figure 2.20: Blossoming. The central spindle turns but the outside coil and the part of the boom that has left the deployer stop moving. The coiled boom unwinds and grows inside the deployer stopping the deployment process.

Figure 2.21 shows the CubeSail deployer before and after blossoming. Before blossoming the booms formed a cylinder with no gaps. As blossoming occurred the coiled booms formed a ring at the outside of the deployer, leaving gaps between the central spindle and the rest of the booms. At this point the deployer stopped deployment and continuing to turn the central spindle would damage the roots of the booms.

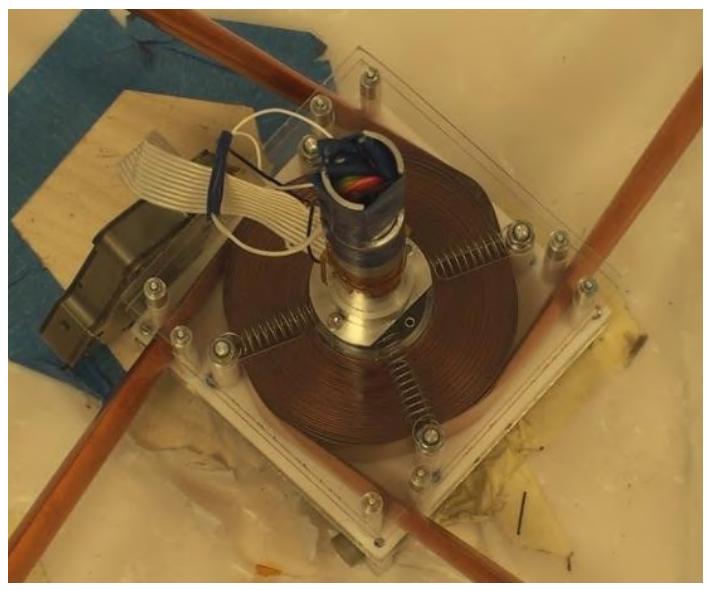

(a) The CubeSail deployer before blossoming.

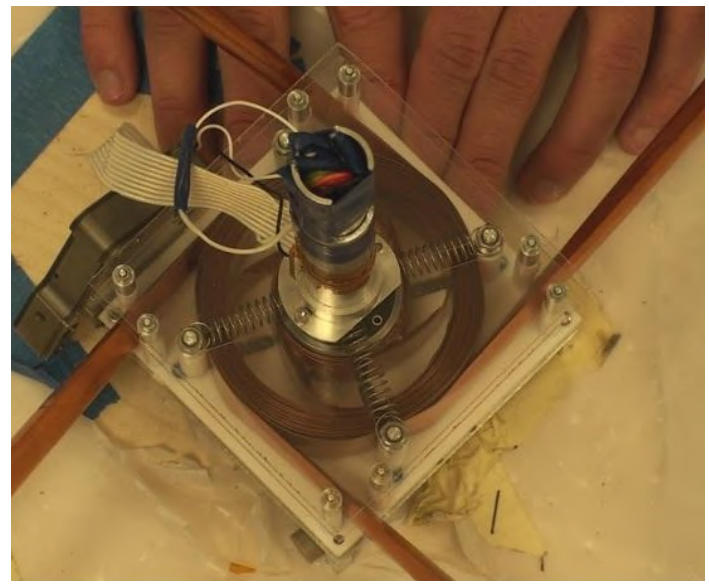

(b) The CubeSail deployer after blossoming.

Figure 2.21: (a) The coiled booms form an almost solid cylinder with no gaps between each coil layer. (b) Blossoming has occurred, some of the boom has deployed. A gap has formed between the central spindle and the boom coil. If the central spindle continues to be turned the roots of the booms will be damaged.

Blossoming is a problem that seems to occur in pusher type deployers. While there have been a number of cases of it occurring (Biddy and Svitek, 2012; Stohlman et al., 2013; Fernandez et al., 2013) there is very little information available on what causes the problem, what parameters effect the tip force a deployer can provide before blossoming, or how to resolve the issue. The aim of 
this $\mathrm{PhD}$ is to clearly define blossoming and to create a model that predicts when blossoming will occur. 


\section{Chapter 3}

\section{Energy method for tip force}

This chapter develops a model that uses the strain energy stored in a coiled tape spring to find the tip force during blossoming. Initially a basic model that simplifies the geometry of a coiled tape spring to a constant radius is used; this is accurate for a small number of coils. The model is developed to include the effects of the boom spiral geometry so the method can be used on a tape spring with a longer length and therefore a significant change in coil radius from the inside to the outside of the coil. The effect the compression springs have on the tip force is also incorporated into the model. An experimental test set-up is used to compare the theory to practical results.

\subsection{Strain energy in a tape spring bend}

A tape spring is one of the most simple deployable booms and is essentially the same as a carpenter's tape measure. If a moments are applied at the ends of a tape spring, it will bend in a similar way to a beam. If the moment is increased above a certain level the tape spring will 'snap through' and form a local bend. This behaviour is elastic and can be carried out without permanent deformation. If a moment is applied so that the concave faces are bought together the snap will be characterised by a torsional twisting mode. This is shown in Figure 3.1 and is termed equal sense bending. If the moment is applied so that the convex faces are bought together the tape spring snaps through suddenly, with a higher moment than for equal sense bending. This is shown in Figure 3.2 and is termed opposite sense bending (Fischer, 1995; Seffen and Pellegrino, 1999).
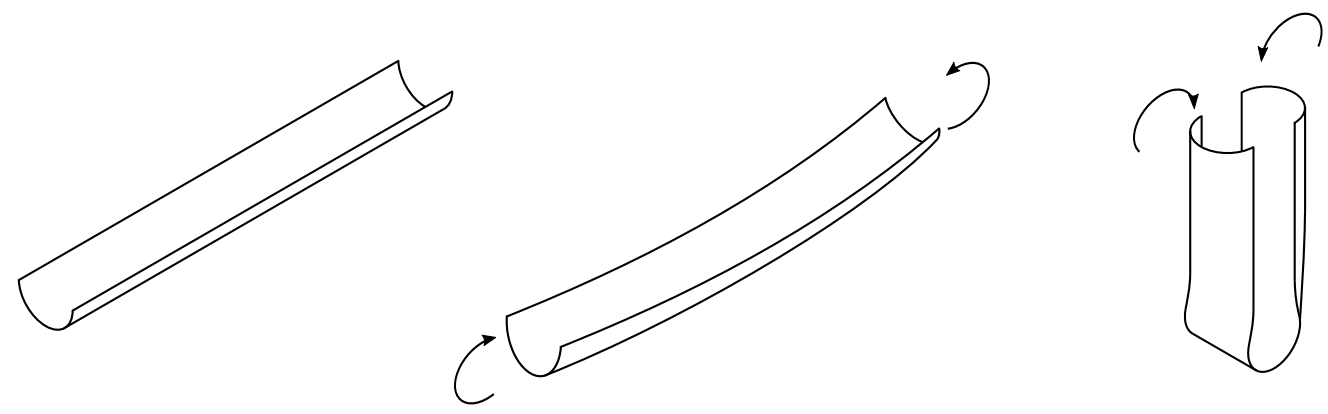

Figure 3.1: Equal sense bending. A moment is applied that brings the concave face of the tape spring together. Initially a curve forms over the whole length of the boom then a local bend forms. 

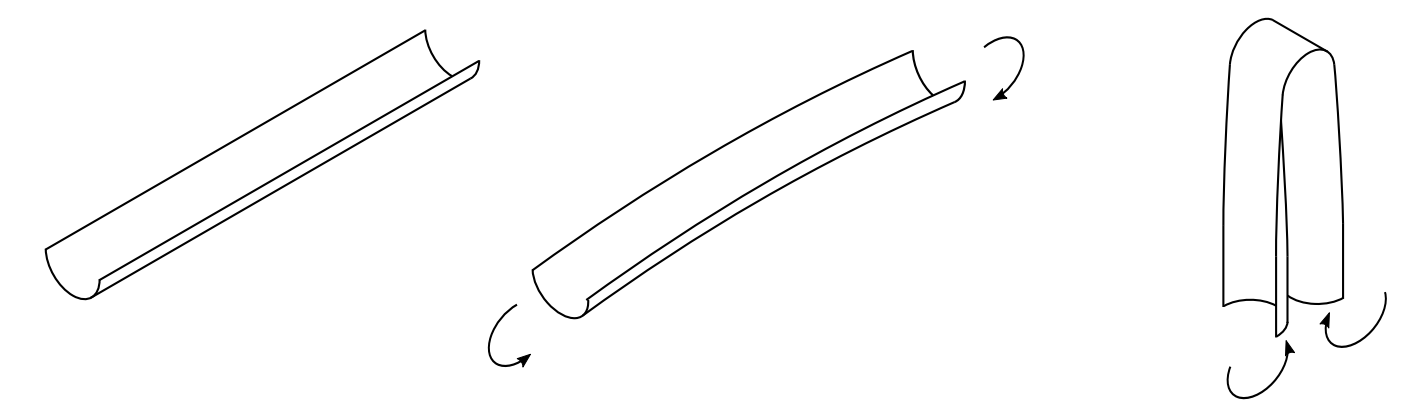

Figure 3.2: Opposite sense bending. A moment is applied that brings the convex face of the boom together. Initially a curve forms over the whole length of the boom. At a critical load the tape spring snaps through and a local bend forms.

The local bend consists of a radius along the length of the tape spring with two transition zones either side, where the tape spring changes from a curved cross section to a flat cross section and back to curved cross section again. This is shown in Figure 3.3.

Figure 3.4 shows the stresses within the tape spring bend. The top part of the diagram shows the whole tape spring and the lower square is a small section of the tape spring bend. (a) shows the initial unstressed state. (b) shows the case of equal sense bending. On the closest face due to the tape spring changing from zero curvature to a bend with a radius $r_{x}$ horizontally along the length of the tape spring, there is a compressive stress state denoted by the large arrows. On the rear face there is tension. On the front face due to the tape spring initially having a curvature $r_{y}$ but now being flat in the vertical direction there is a tensile stress denoted by the small arrows. On the rear surface there is compression vertically. (c) shows the case of opposite sense bending. On the closest face there is a compressive stress state both horizontally and vertically. On the rear face there is a tensile stress state both horizontally and vertically. A tape spring made from an isotropic material will have a bend radius $\left(r_{x}\right)$ the same as the cross section radius $\left(r_{y}\right)$ of the tape spring. This is due to the strain energy stored in the bend being at a minimum when the radius is the same as the unstressed cross section i.e. $r_{x}=r_{y}$ in Figure 3.3. This can be shown using the following strain energy method (Calladine, 1988).

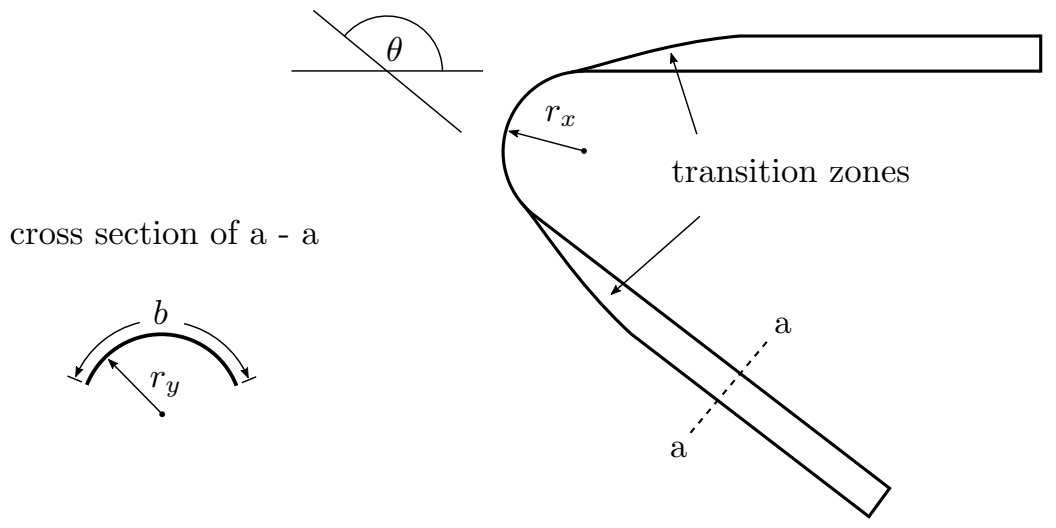

Figure 3.3: A tape spring with local bend. The cross section radius of curvature $r_{y}$ is the same as the bend radius $r_{x}$. 

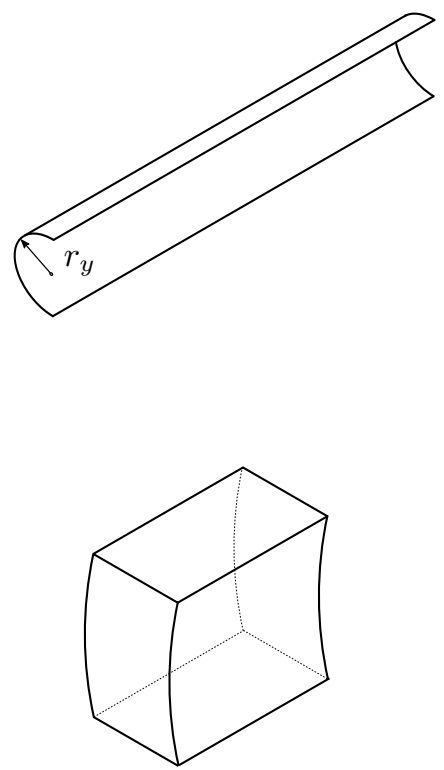

(a)

Figure 3.4: The stress states within the bent part of the boom. condition. (b) Equal sense bending. (c) Opposite sense bending.
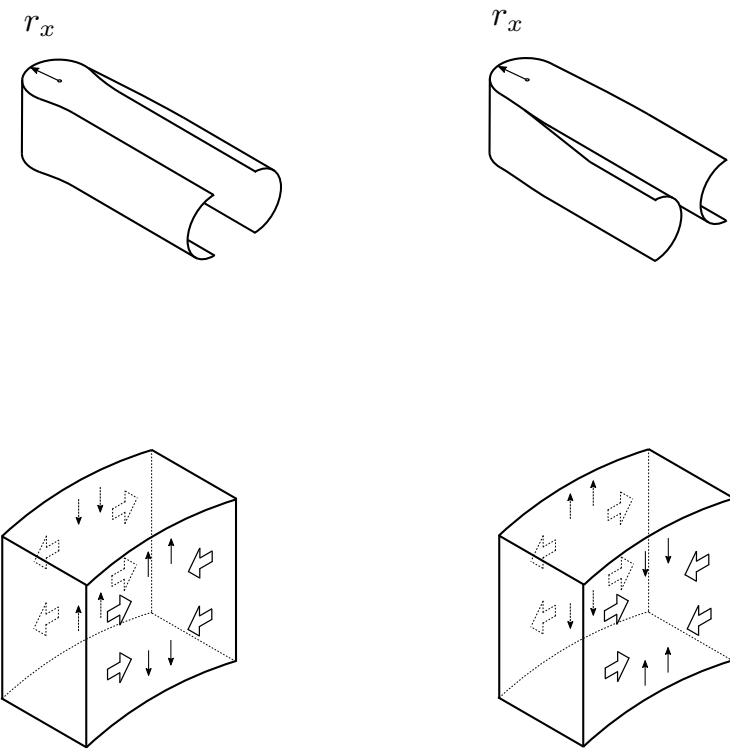

(b)

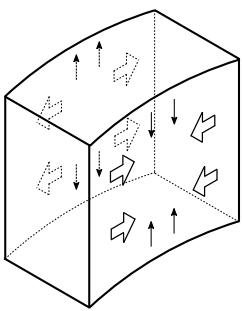

(c)

The strain energy stored in a thin plate in pure bending is given by Equation 3.1 (Timoshenko et al., 1959).

$$
U=\frac{1}{2} D A\left[\left(\Delta \kappa_{x}\right)^{2}+\left(\Delta \kappa_{y}\right)^{2}+2 \nu\left(\Delta \kappa_{x} \Delta \kappa_{y}\right)\right]
$$

$D$ is the flexural rigidity of a plate, as shown in Equation 3.2. $A$ is the planform area of the plate midsection, $\kappa_{x}$ and $\kappa_{y}$ are the curvatures in the $x$ and $y$ directions respectively, and $\nu$ is Poisson's ratio. $\left(\kappa_{x}=\frac{1}{r_{x}}\right.$ and $\left.\kappa_{y}=\frac{1}{r_{y}}\right)$.

$$
D=\frac{E t^{3}}{12\left(1-\nu^{2}\right)}
$$

$E$ is the Young's modulus of the material, $t$ is the tape spring thickness and $\nu$ is Possion's ratio. Equation 3.1 can be used to describe the changes in strain energy in the curved part of the tape spring shown in Figure 3.3. The $x$ direction is along the length of the tape spring and the $y$ direction is across the width. The area is the curved section denoted by $r_{x}$ and $\theta$. The material in the bend area has undergone changes in curvature. Initially the the lateral curvature was $\frac{1}{r_{y}}$ but now is zero. The longitudinal curvature was initially zero but is now $\frac{1}{r_{x}}$. 
Hence for opposite sense bending the changes in curvature are:

$$
\left(\Delta \kappa_{x}, \Delta \kappa_{y}\right)=\left(\frac{1}{r_{x}}, \frac{1}{r_{y}}\right)
$$

The transition zones can be ignored as they are a constant feature once the fold has formed. Any change in the angle of the bend will move the transition zones along the length of the tape spring but will not change their shape, hence there is no change in strain energy within the transition zones.

Therefore the strain energy in the bend of a tape spring with opposite sense bending is:

$$
U_{o p}=\frac{D A}{2}\left[\frac{1}{r_{x}^{2}}+\frac{1}{r_{y}^{2}}+\frac{2 \nu}{r_{x} r_{y}}\right]
$$

Where the area is given by:

$$
A=b r_{x} \theta
$$

The width of the tape spring is denoted by $b, r_{x}$ is the radius of the tape spring bend and $\theta$ is the angle subtended by the bend, as shown in Figure 3.3. Substituting Equation 3.5 into Equation 3.4 gives:

$$
U_{o p}=\frac{D b r_{x} \theta}{2}\left[\frac{1}{r_{x}^{2}}+\frac{1}{r_{y}^{2}}+\frac{2 \nu}{r_{x} r_{y}}\right]
$$

This cancels to give the strain energy in the bent part of the tape spring:

$$
U_{o p}=\frac{D b \theta}{2}\left[\frac{1}{r_{x}}+\frac{r_{x}}{r_{y}^{2}}+\frac{2 \nu}{r_{y}}\right]
$$

If Equation 3.7 is plotted with a range of different bend radii a strain energy minimum can be seen when $r_{x}=r_{y}$ as shown in Figure 3.5. The tape spring dimensions chosen for plotting are of a standard size steel carpenter's tape measure and are representative of the tape spring sizes used within CubeSats. 


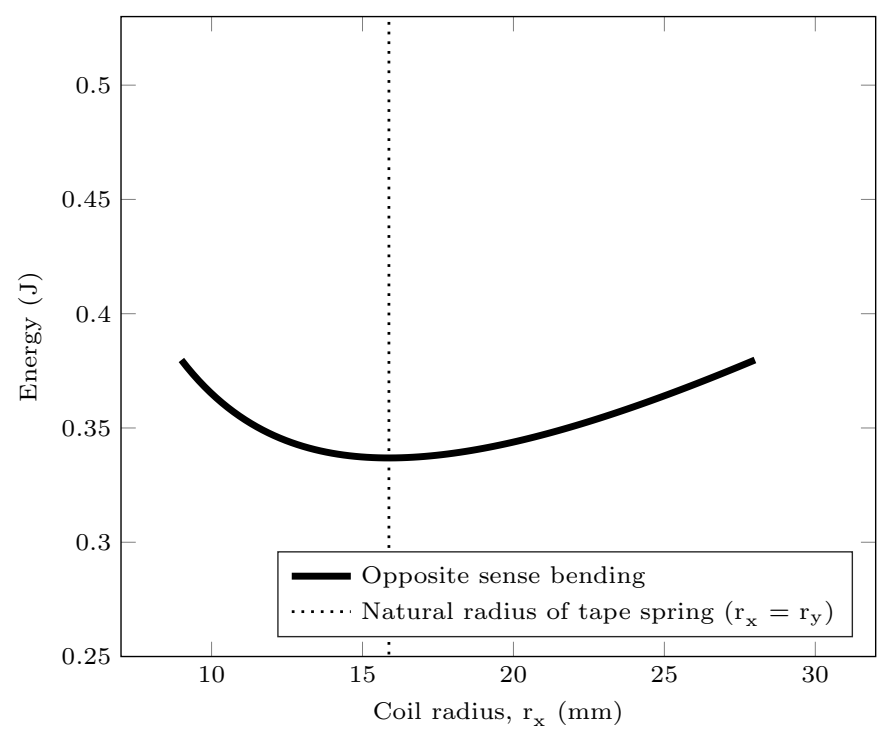

Figure 3.5: The energy in the curved section of a tape spring at different radii for opposite sense bending, the natural radius is also shown. Tape spring: width $=31.8 \mathrm{~mm}$, thickness $=0.13 \mathrm{~mm}$, Young's modulus $=205 \mathrm{GPa}$, angle of bend $=180^{\circ}, r_{y}=15.9 \mathrm{~mm}$.

The energy minimum can also be found by taking the first derivative with respect to coil radius of Equation 3.7 which gives:

$$
\frac{d U}{d r_{x}}=\frac{D b \theta}{2}\left[\frac{1}{r_{y}^{2}}-\frac{1}{r_{x}^{2}}\right]
$$

When the bend radius matches the natural radius of the tape spring $\left(r_{x}=r_{y}\right)$ the gradient of the energy is zero $\left(\frac{d U}{d r_{x}}=0\right)$. The second derivative of Equation 3.7 is positive as shown in Equation 3.9. This is another way of confirming that there is an energy minimum when $r_{x}=r_{y}$.

$$
\frac{d^{2} U}{d r_{x}^{2}}=\frac{D b \theta}{2}\left[\frac{2}{r_{x}^{3}}\right]
$$

The same analysis would work for equal sense bending, the difference being a reversal of the sign of curvature for $\kappa_{x}$. This leads to Equation 3.10 which shows the energy stored in the bent part of the tape spring for equal sense bending.

$$
U_{e q}=\frac{D b \theta}{2}\left[\frac{1}{r_{x}}+\frac{r_{x}}{r_{y}^{2}}-\frac{2 \nu}{r_{y}}\right]
$$

Figure 3.6 compares the strain energies in the bent part of the tape spring for equal and opposite sense bending. Both cases follow the same gradient and have the energy minimum at the same radius, however the opposite sense bending case has a higher strain energy due to the Poisson's ratio effect. 


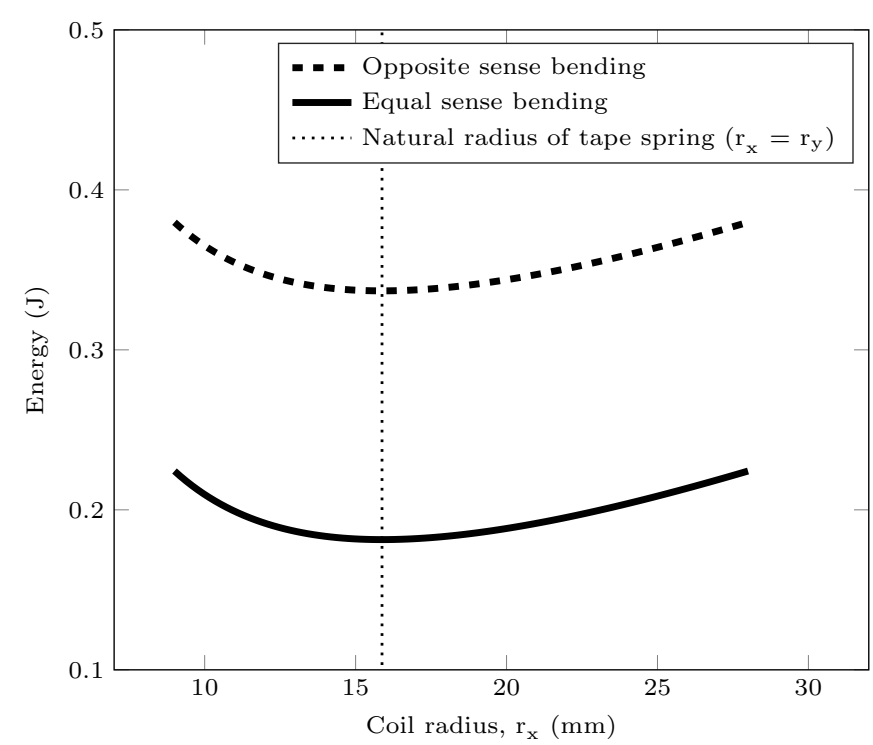

Figure 3.6: The energy in the curved section of a tape spring at different radii for equal and opposite sense bending, the natural radius is also shown. Tape spring: width $=31.8 \mathrm{~mm}$, thickness $=0.13 \mathrm{~mm}$, Young's modulus $=205 \mathrm{GPa}$, angle of bend $=180^{\circ}, r_{y}=15.9 \mathrm{~mm}$.

This analysis shows that if a tape spring has a bend with a constant angle it will always try and keep the bend radius the same as the natural radius of the tape spring as this will allow it to remain at its lowest strain energy. To cause the bend to move to a larger or smaller radius, a force will have to be applied and when the force is removed the original bend radius will be restored. This can be verified by hand with a carpenter's tape measure.

\subsection{Constant radius method}

A coiled tape spring can be thought of as a tape spring with a bend angle of more than $360^{\circ}$; therefore when a tape spring is coiled with a small number of complete turns, the coil radius will match the natural radius of the tape spring, as this will minimise the strain energy in the coiled part. There will be a small change in coil radius from the inside to the outside of the coil due to the thickness of the tape spring. For a small number of coils an approximation to the coil having a constant radius can be made as the tape thickness is significantly smaller than the coil radius. A bent or coiled tape spring will always try and uncoil as this will allow it to obtain a lower strain energy state. If a coiled tape spring is constrained so that its subtended angle can not change but its length is allowed to extend or contract, it will act as a spring, with the coiled part of the tape spring trying to return to the energy minimum, this is shown in Figure 3.7. The tape spring is constrained at its inside end and stopped from uncoiling by a roller. This allows the radius of the tape spring coil to increase or decrease but the coil will maintain its subtended angle. Part (a) of Figure 3.7 shows the coil in equilibrium as the coil radius is the same as the tape spring's natural radius, hence there is no tip force. Part (b) of Figure 3.7 shows a force $\left(\mathrm{F}_{\mathrm{app}}\right)$ has been applied to the tip of the tape spring, pulling the end away from the coil. This has reduced the coil radius below the natural radius of the tape spring which creates a force $\left(\mathrm{F}_{\text {tip }}\right)$ in the coil, opposing the applied force. Part (c) of Figure 3.7 shows a force being applied towards the coil, increasing the radius of the coil above the natural radius of the tape spring. An equal and opposite force is created by the coiled tape spring. When blossoming occurs the coiled tape spring is in a situation similar to part (c) of Figure 3.7. Instead of the tip of the boom being pushed towards the coil, the tip is held stationary and the central spindle is rotated causing the coil radius to increase. By finding the force the coiled boom will exert as the coil radius increases, an indication of how much 
tip force a coiled boom will resist before blossoming can be found.

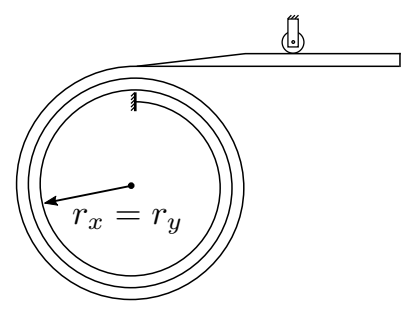

(a)

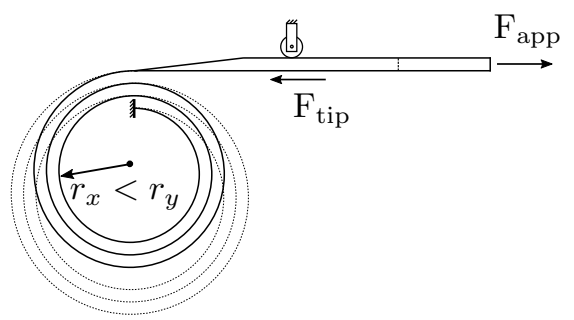

(b)

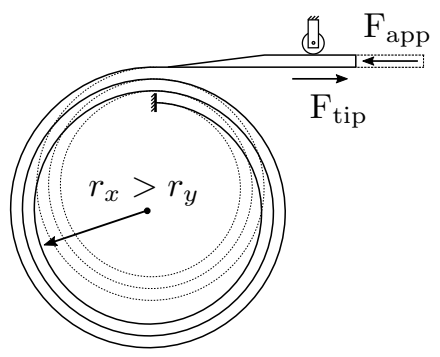

(c)

Figure 3.7: A coiled tape spring acting as a spring in extension and compression. The dotted lines in (b) and (c) denote the original coil position. (a) The coil in equilibrium. (b) The coil at a smaller radius than the tape spring's natural radius. A force has to be applied to maintain the position. (c) The coil at a larger radius than the tape spring's natural radius. A force has to be applied to maintain the position.

As the boom tip is extended away from the coil or compressed towards it, the length of the boom in the coil changes. If the angle subtended by the coil is kept constant, the radius of the coil will change. This is shown in Figure 3.8. Initially the length of the boom in the coil is $r_{x 1}$ (initial coil radius) multiplied by $\theta$ (angle subtended by the coil). The deployed part of the boom also has a certain length. In Figure 3.8(b) the boom is pulled away from the coil. This increases the length of the part of the boom that is deployed and decreases the length of the boom in the coil, which is now given by $r_{x 2}$ (final coil radius) multiplied by $\theta$. This gives the relationship between the length extended $(\Delta L)$ by the tip and the change in coil radius:

$$
\Delta L=\left(r_{x 1}-r_{x 2}\right) \theta
$$

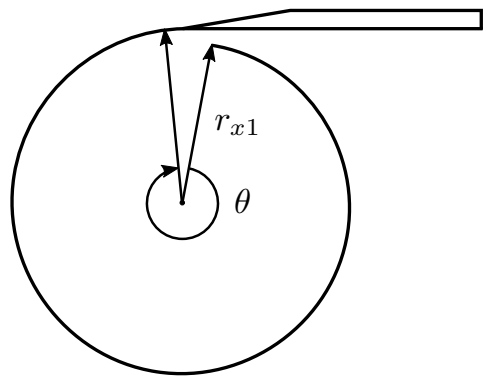

(a)

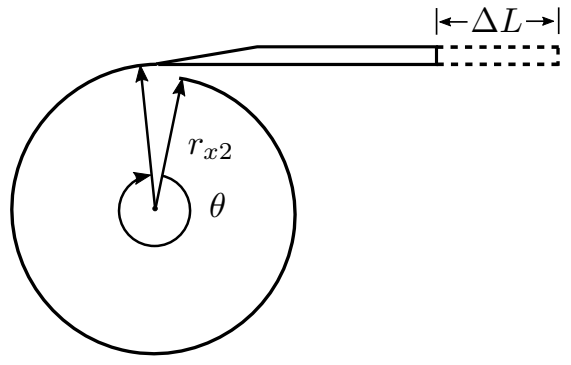

(b)

Figure 3.8: The change in coil length. (a) The initial condition with the length of the boom in the coil being given by $r_{x 1} \theta$. (b) The part of the boom that is in the deployed state has been extended by the length $\Delta L$. This decreases the length of the boom in the coil which is now given by $r_{x 2} \theta$.

By using the previous equations for the strain energy in the bent part of the tape spring and extending this to a small number of coils, the boom tip force can be found when the coil is forced above or below the natural radius. Equation 3.8 shows the rate of change of strain energy as the radius changes. The relationship between the coil radius and the length of the boom allows Equation 3.8 to be changed into a force for a given extension of the tape spring tip as shown in 
Equation 3.12.

$$
\frac{d U}{d r_{x}} \frac{d r_{x}}{d L}=\frac{d U}{d L} \quad L=r_{x} \theta \quad \Rightarrow \quad \frac{d r_{x}}{d L}=\frac{r_{x}}{L}=\frac{1}{\theta}
$$

In this case $L$ is the length of the tape spring, $r_{x}$ is the coil radius and $\theta$ is the angle subtended. Combining Equations 3.8 and 3.12 gives Equation 3.13 which gives the tip force for a given coil radius.

$$
F_{t i p}=\frac{d U}{d L}=\frac{D b}{2}\left[\frac{1}{r_{y}^{2}}-\frac{1}{r_{x}^{2}}\right]
$$

\subsubsection{Compression spring analogy}

The method of using the rate of change of strain energy with respect to boom tip compression is analogous to a normal compression spring. A linear compression spring can be described by Equation 3.14.

$$
F=k x
$$

Where $F$ is the force, $k$ is the spring stiffness and $x$ is the spring displacement. Work done to compress a spring is force multiplied by distance. This work done is stored as strain energy $(V)$ in the spring and is given by $F x$. In the case of a spring with a constant stiffness can be given by Equation 3.15.

$$
V=F x \rightarrow V=\int_{0}^{x} F d x \rightarrow V=\int_{0}^{x} k x d x \quad \rightarrow \quad V=\frac{k x^{2}}{2}
$$

In the reverse process, the derivative of the strain energy with respect to length compressed gives the force, as shown in Equation 3.16.

$$
V=\frac{k x^{2}}{2} \rightarrow \frac{d V}{d x}=k x=F
$$

This is shown graphically in Figure 3.9. The force is the gradient of the strain energy and the strain energy is the area under the force displacement line. In the case of a coiled tape spring the strain energy at different tip displacements is known. The force can be found by taking the derivative of the strain energy with respect to tip length displaced $\left(\frac{d U}{d L}\right)$. 


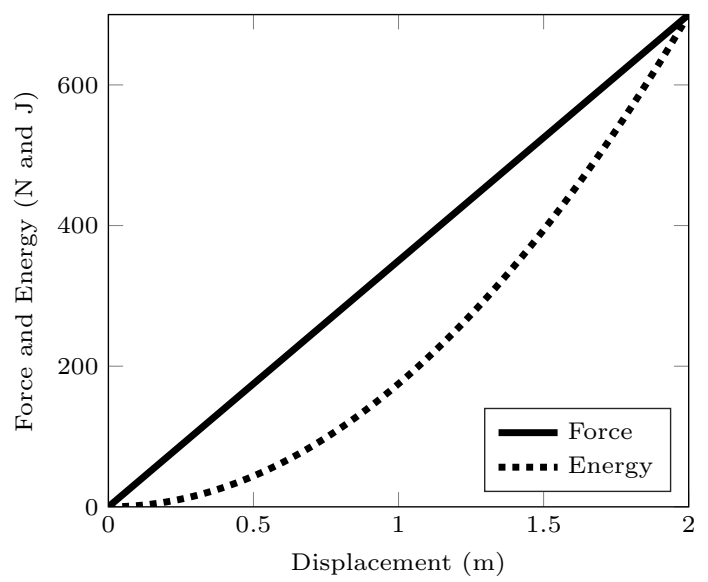

Figure 3.9: The force and stored strain energy for a linear compression spring with a spring stiffness of $350 \mathrm{~N} / \mathrm{m}$.

\subsubsection{Energy and force plots}

Figure 3.10 uses Equation 3.7 to plot the strain energy against the coil radius. Figure 3.11(a) plots the same strain energy in the coil using Equation 3.7 but plots it against the tip displacement, using Equation 3.11. Figure 3.11(b) shows the tip force for the same tape spring over a range of tip extension, plotted using Equations 3.11 and 3.13.

The point of zero tip displacement in Figure 3.11(b) corresponds to the coil being at the natural radius in Figure 3.10 and at zero tip displacement in Figure 3.11(a) where the strain energy in the coil is at a minimum. The boom tip being displaced positively in Figure 3.11(b) corresponds to the coil radius increasing above the natural radius in Figure 3.10, increasing the strain energy. The boom tip being extended in Figure 3.11(b) (or negative tip displacement) corresponds to the coil radius becoming smaller than the natural radius, again increasing the strain energy in the coil. The gradient of the strain energy in Figure 3.11(a) ( $\left.\frac{d U}{d l}\right)$ gives the force in Figure 3.11(b), this is a method of visualising Equations 3.7 and 3.13.

Table 3.1: Table of tape spring properties used for some of the following figures.

\begin{tabular}{|l|l|l|l|l|}
\hline width $-\mathrm{b}(\mathrm{mm})$ & thickness - t $(\mathrm{mm})$ & tape spring natural radius $-r_{y}(\mathrm{~mm})$ & $\mathrm{E}(\mathrm{GPa})$ & $\nu$ \\
\hline \hline 31.8 & 0.13 & 15.9 & 205 & 0.3 \\
\hline
\end{tabular}




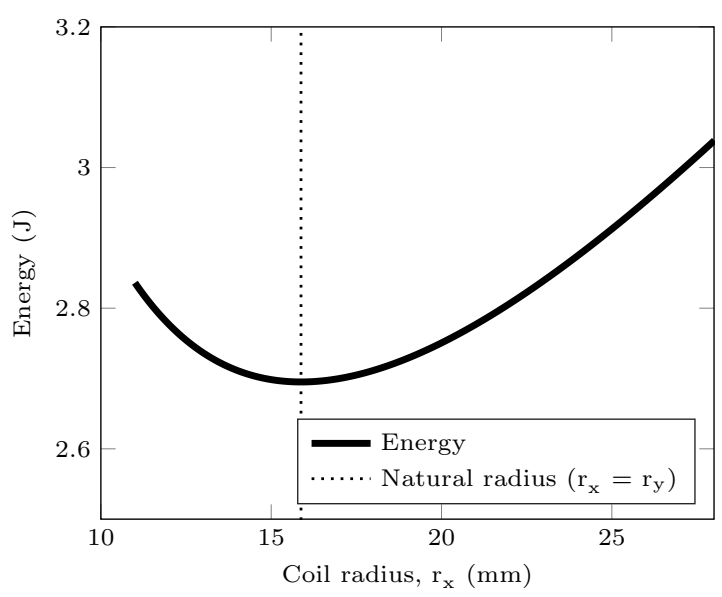

Figure 3.10: Strain energy stored in the coiled part of the boom held at different radii. There are 4 turns in the coil. Boom dimensions are shown in Table 3.1.

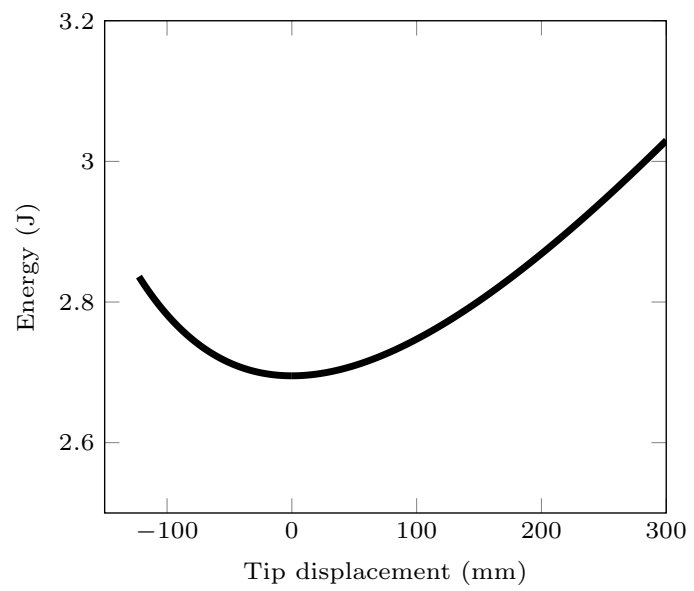

(a) Strain energy.

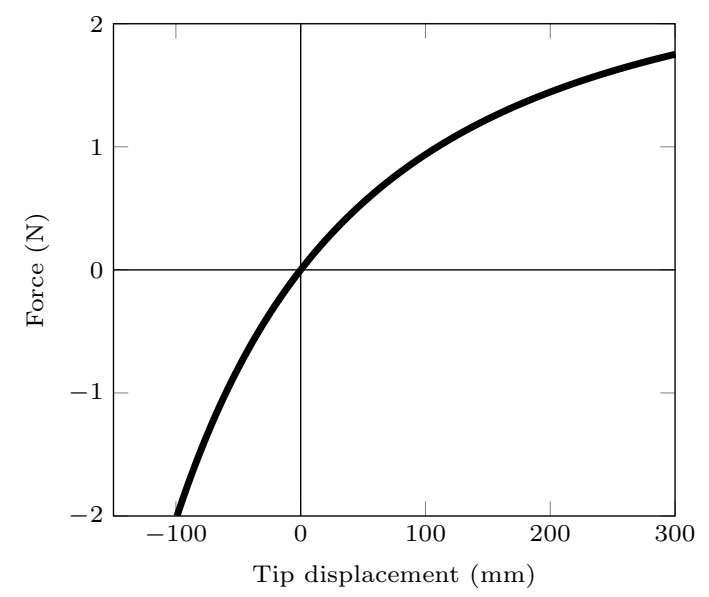

(b) Tip force.

Figure 3.11: Strain energy and tip force for a coil that has its tip extended and compressed. There are 4 turns in the coil. Boom dimensions are shown in Table 3.1.

When the tip of the boom is moved away from the coil (negative tip displacement) the force increases rapidly as shown in Figure 3.11(b). This is due increase of strain energy from the tighter coil curvature. When the tip is moved towards the coiled tape spring, increasing the radius of the coil, the force increases but with a decreasing gradient as the coil becomes larger. As the coil increases in size, the gradient of the force decreases until it asymptotes at a maximum. This theoretical maximum is when there is no coil curvature $\left(r_{x} \rightarrow \infty\right)$ as is indicated by Equation 3.13. This is shown in Figure 3.12; the maximum force line is calculated by setting $\frac{1}{r_{x}}=0$ in Equation 3.13. This maximum force is determined by the Young's modulus and Poisson's ratio of the tape spring material, and the thickness and width of the tape spring. 


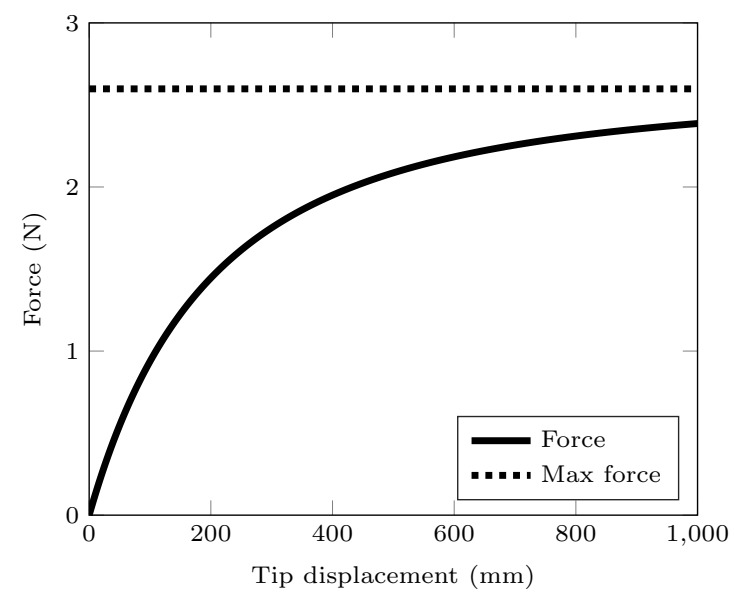

Figure 3.12: The boom tip force increasing towards a maximum tip force. There are 4 turns in the coil. Boom dimensions are shown in Table 3.1.

As Equation 3.13 shows, the tip force only depends on the radius of the coil and not on how many turns there are in the coil; however there is a relationship between the number of coils and the change in coil radius for a given extension or compression of the tip of the boom. This is shown in Equation 3.11. If there are a small number of coils (small value of $\theta$ ) the coil radius will change quickly in relation to the tip compression or extension, this leads to a more rapid increase in tip force. If there are a greater number of coils (large value of $\theta$ ) there will be a smaller change in coil radius, this leads to a more gradual increase in tip force. If a coiled tape spring is thought of as a spring, changing the number of turns in the coil will have the effect of changing the spring stiffness as shown in Figure 3.13.

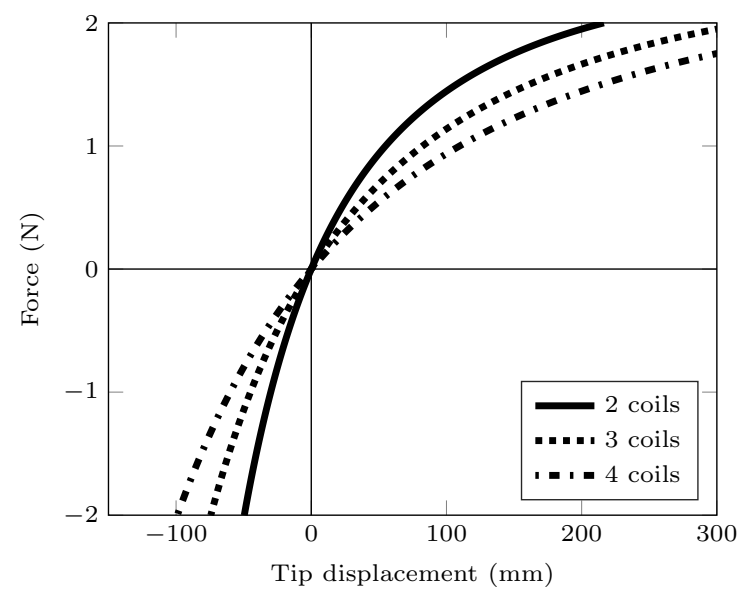

Figure 3.13: Comparison between 2, 3 and 4 coils. There are 4 turns in the coil. Boom dimensions are shown in Table 3.1 .

\subsubsection{Simple experimental test set-up}

To confirm this theory with a small number of coils a simple test apparatus was manufactured as shown in Figure 3.14(c). A coiled boom is constrained at the inside end and its subtended angle is fixed by another roller, allowing the coil radius to increase and decrease in size but to maintain a constant bend angle. A further roller is added just behind the main attachment point to ensure that all the coils maintained the same radius as without this only the outside coil would move. In CubeSail type deployers there are four compression rollers that fulfil a similar role. Figure 3.14(a) shows a schematic of the test set-up. The tape spring is constrained in an Instron mechanical testing machine with the tip held in the load cell. The cross head is moved up and down to create 
the extension and compression of the tip of the tape spring while the load cell measures the tip force. An Instron 2525 series $100 \mathrm{~N}$ drop through static load cell was used. The cross head was moved at rate of $1.6 \mathrm{~mm} / \mathrm{second}$ and data was recorded at $10 \mathrm{~Hz}$. The results shown in Figure 3.15 and Figure 3.16 are typical and second set of results is shown in Appendix A.

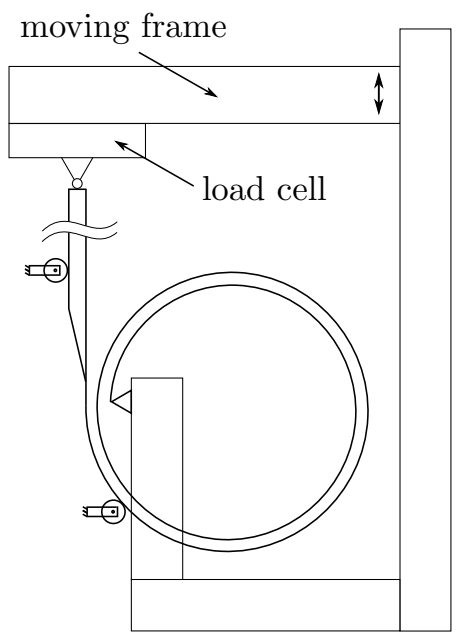

(a) Schematic of the test rig in the instron test machine.

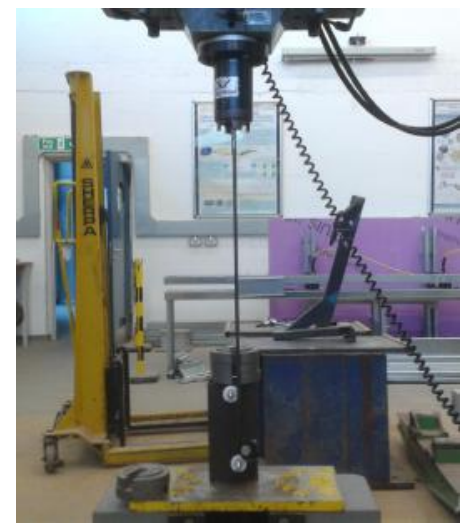

(b) Photo of the test rig in the instron test machine.

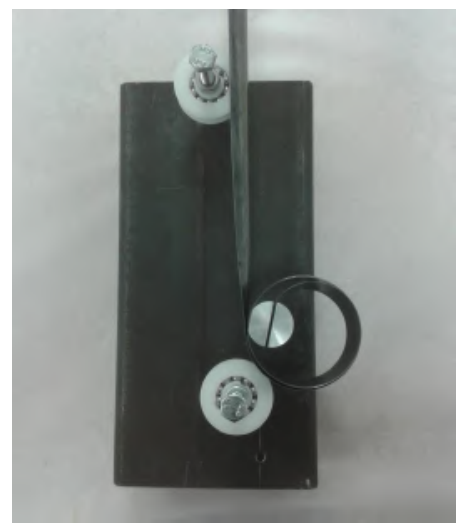

(c) Photo of the test rig.

Figure 3.14: Simple experimental test set-up.

The results from tests for two, three and four coils are shown in Figures 3.15 and 3.16 below. The experimental results recorded match quite closely to the forces expected from the theory. The experimental results follow the higher force gradient for the smaller number of coils and the lower gradient for a greater number of coils. Although the experimental and theoretical results match fairly well there seems to be quite a bit of oscillation in the experimental results. This is though to be because of the friction between the different coil layers as they slid past one another. It seemed that not all of the coils were in contact with one another continuously through out the boom movement, this may have given rise to the variations in measured tip force. With the changes in force due to the friction between the layers of tape spring taken into account, the results seem to match quite well.

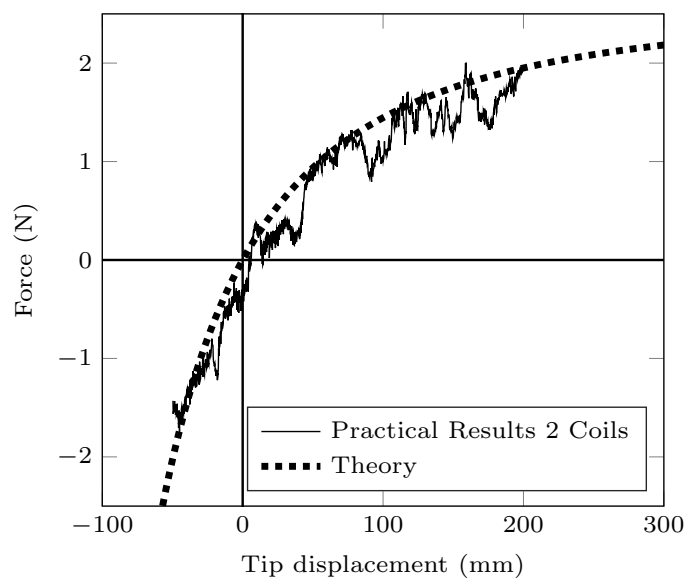

(a) 2 coils

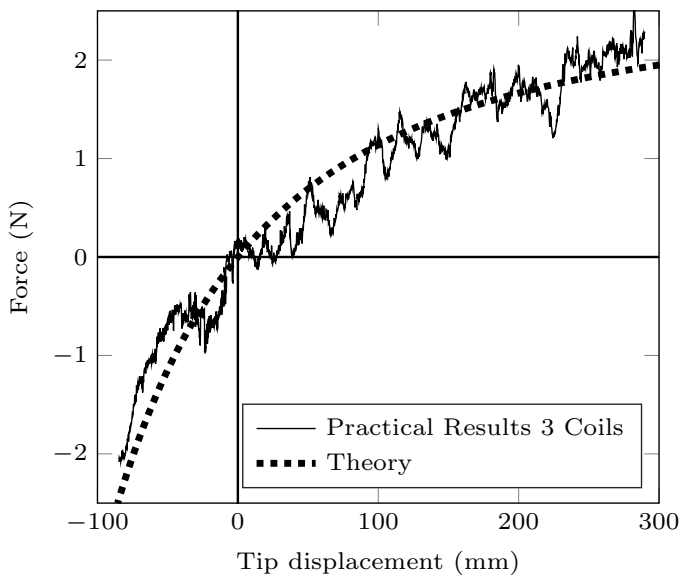

(b) 3 coils

Figure 3.15: Results for 2 and 3 coils. Boom dimensions are shown in Table 3.1. 


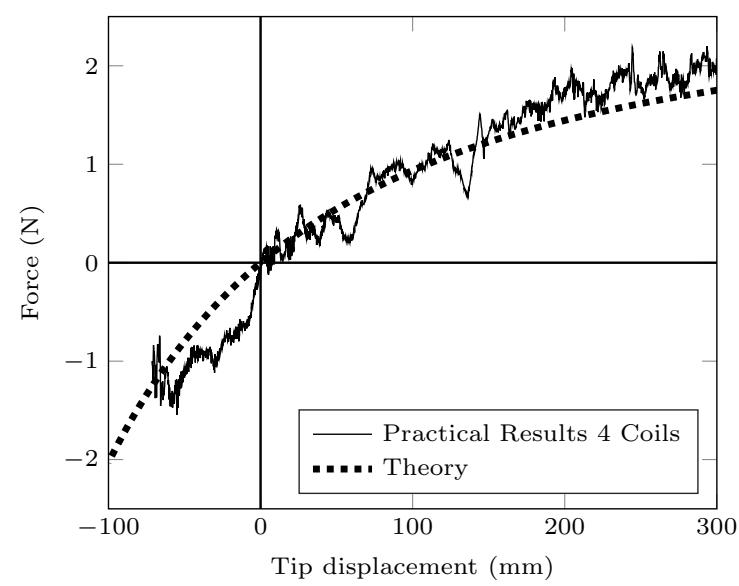

Figure 3.16: Results for 4 coils. Boom dimensions are shown in Table 3.1.

\subsection{Spiral geometry}

When a coiled tape spring has a small number of coils and is sufficiently thin (boom thickness approximately 100 times smaller than the coil radius), its coil radius can be approximated as constant along its length. This is due to the change in radius from the start to the end of the tape spring being much smaller than the coil radius. When there are a large number of coils it is necessary to take in to account the change in the coil radius along the length of the boom. This is shown in Figure 3.17; the radius increases from the central spindle $\left(r_{x 1}\right)$ to the outside of the coil $\left(r_{x 2}\right)$. In the design of solar sailing missions such as CubeSail, which require efficient use of space, the coil radius may change from $\approx 15 \mathrm{~mm}$ at the inside of the coil to $\approx 45 \mathrm{~mm}$ at the outside (Lappas et al., 2011). In the case of a long tape spring with a significant change in radius along its length there will be a noticeable difference in the strain energy if it is modelled as having a constant radius compared to it being modelled as having a changing radius. By taking into account the change in radius of the tape spring along its length, a more accurate strain energy can be calculated and therefore a more accurate tip force can be found.

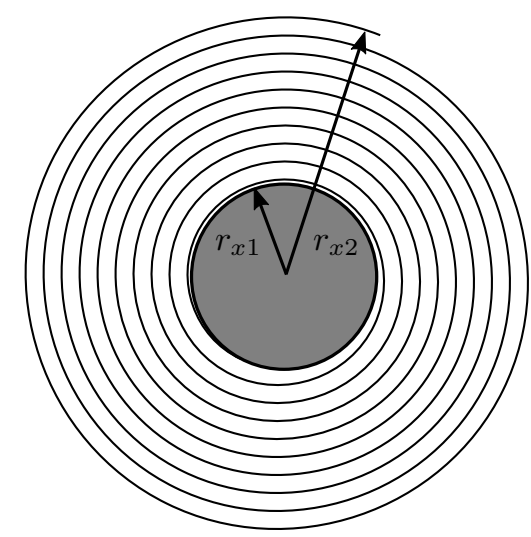

Figure 3.17: Top view of a coiled tape spring showing how the coil radius varies along the boom length.

A coiled boom or a number of co-coiled booms can be approximated to have the same shape as an Archimedes' spiral. This is a spiral that has a constant distance between each successive layer or coil. For an Archimedes' spiral the radius $(r)$, curvature $(\kappa)$ and length $(s)$ for a given angle subtended $(\theta)$ are given in Equations 3.17, 3.18 and 3.19 (Weisstein, 2016) and are shown in 
Figure 3.18. If the angle subtended $\theta$ is in radians then $a$ is the thickness between each layer of coiled boom divided by $2 \pi$ i.e. $a=\frac{t}{2 \pi}$. $t$ is the distance between each coil layer.

$$
r(\theta)=a \theta
$$

$$
\kappa(\theta)=\frac{2+\theta^{2}}{a\left(1+\theta^{2}\right)^{\frac{3}{2}}}
$$

$$
s(\theta)=\frac{1}{2} a\left(\theta \sqrt{1+\theta^{2}}+\sinh ^{-1}(\theta)\right)
$$

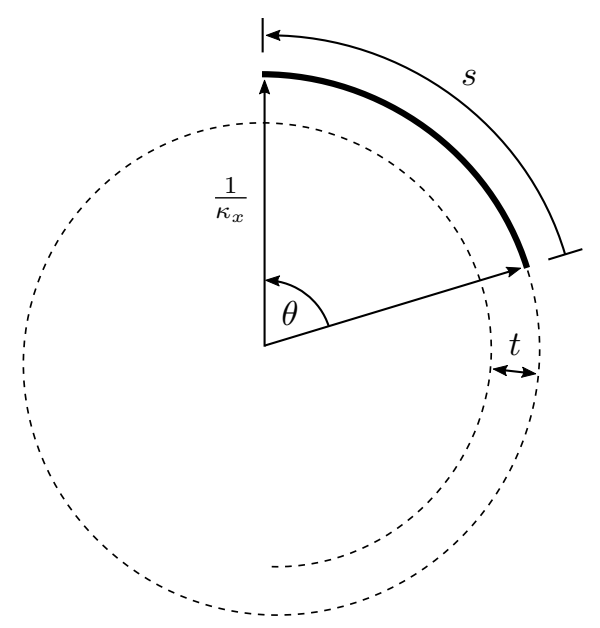

Figure 3.18: The dimensioning of a spiral. $s$ is the length, $t$ is the thickness between each layer $\theta$ is the angle subtended and $\frac{1}{\kappa_{x}}$ is an approximation to the instantaneous radius.

Using Equations 3.17, 3.18 and 3.19 the strain energy stored in a coiled boom can be found. MATLAB (Documentation, 2005) can be used to create a plot of the curvature along the length of the boom, as shown in Figure 3.19. The method is detailed in Appendix B using the scripts coillength and inverscoillength. The zero position is where the coil attaches to the central spindle and therefore has the highest curvature. Moving along the length of the boom the curvature decreases until it reaches the tip. Using a trapizodial method, the area under the curvature length plot can be used along with Equation 3.7 to find the strain energy stored in the coil, taking into account the change in curvature along the length of the boom. 


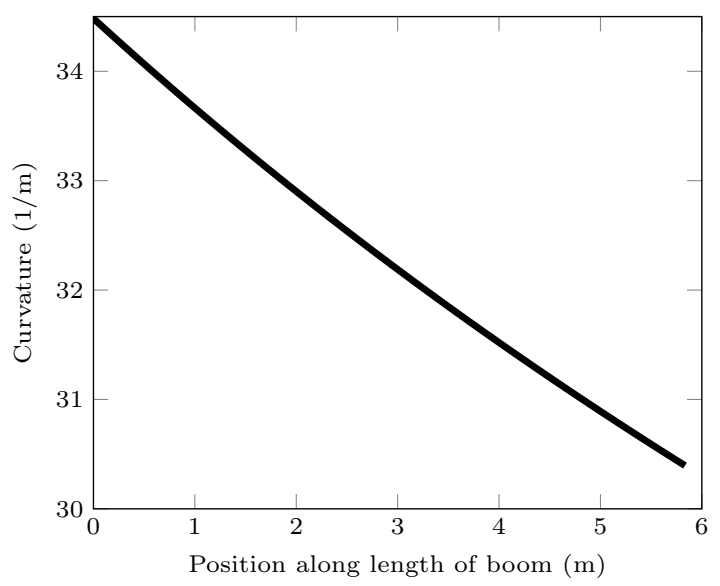

Figure 3.19: The curvature along the length of the boom. The radius at the inside of the coil is $29 \mathrm{~mm}$, the radius at the outside of the coil is $33 \mathrm{~mm}$, the thickness of the boom is $0.13 \mathrm{~mm}$, and number of turns in the coils is 30 .

The difference in strain energy between a coiled tape spring that has been modelled as a spiral and one that has been modelled as having a constant radius is shown in Figure 3.20. In this figure the two methods have been compared by setting the inside radius of the coil using the spiral method to the same radius as the constant radius method and by having the same number of coils. It shows that while the two curves seem to follow similar gradients the curve that uses the spiral geometry has been shifted to the left and has its energy minimum at a smaller coil radius than the curve using the constant radius simplification. This is due to most of the coil in the spiral geometry being at a larger radius than the natural radius of the tape spring and hence being at a higher strain energy. This also explains the strain energy minimum being at a smaller coil radius for the spiral method as opposed to the circle method. The lowest strain energy possible for a spiral would be found when some of the coil is at a radius less than the natural radius of the tape spring and some at a greater radius as Figure 3.21 shows.

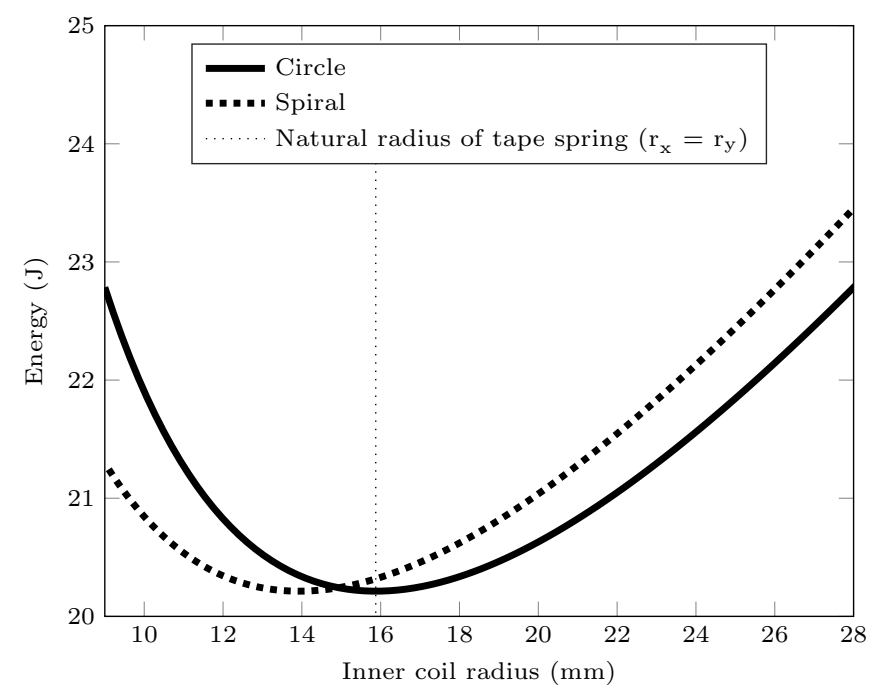

Figure 3.20: A comparison between the circle and spiral method of finding the strain energy stored in the coil. The vertical line denotes the boom's natural radius. There are 30 turns in the coil. Boom dimensions are shown in Table 3.1. 


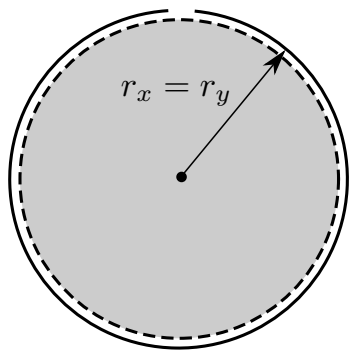

(a)

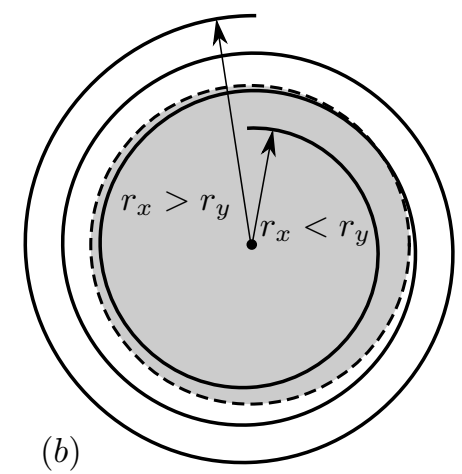

$(b)$

Figure 3.21: A comparison of the strain energy minimum using the spiral geometry and the simplification to the coil having a constant radius. The central spindle is the grey dotted circle and has a radius the same as the natural radius of the tape spring. (a) The coil radius is approximated to having a constant radius, the energy minimum is when $r_{x}=r_{y}$. (b) Shows the spiral method. The strain energy is at a minimum when some of the coil is as a radius that is smaller than the tape spring's natural radius and some of the coil is at a radius that is larger than the tape spring's natural radius.

The shifting of the strain energy curve to the left when the spiral model is used instead of the simplification of the coiled boom to a circle means that the tip force will also change. Just as before, the tip force can be found by finding the gradient of the strain energy with respect to length change of the boom tip. Instead of using Equation 3.13, MATLAB is used to find the strain energy stored in the coil using spiral geometry. The general method used is shown in Figure 3.22. This method is used to find the tip force and central spindle torque with and without compression roller springs. Details of the scripts coillength, inversecoillength, coilenergy and springenergy can be found in Appendix B. The initial values of the inner coil radius and angle subtended by the coil are given to coillength and inversecoillength. From these scripts the curvature along the length of the boom and the displacement of the compression springs are found. The scripts coilenergy and springenergy find the amount of strain energy stored in the boom coil and the compression springs, this is recorded. The initial coil angle is reduced to give the action of blossoming. This loop is repeated for the angle of blossoming required. The strain energy in the boom coil and compression springs is stored at each step. The gradient of the strain energy against length extended $\left(\frac{d U}{d L}\right)$ gives the tip force and the gradient of the strain energy against angle rotated $\left(\frac{d U}{d \theta}\right)$ gives the central spindle torque. 


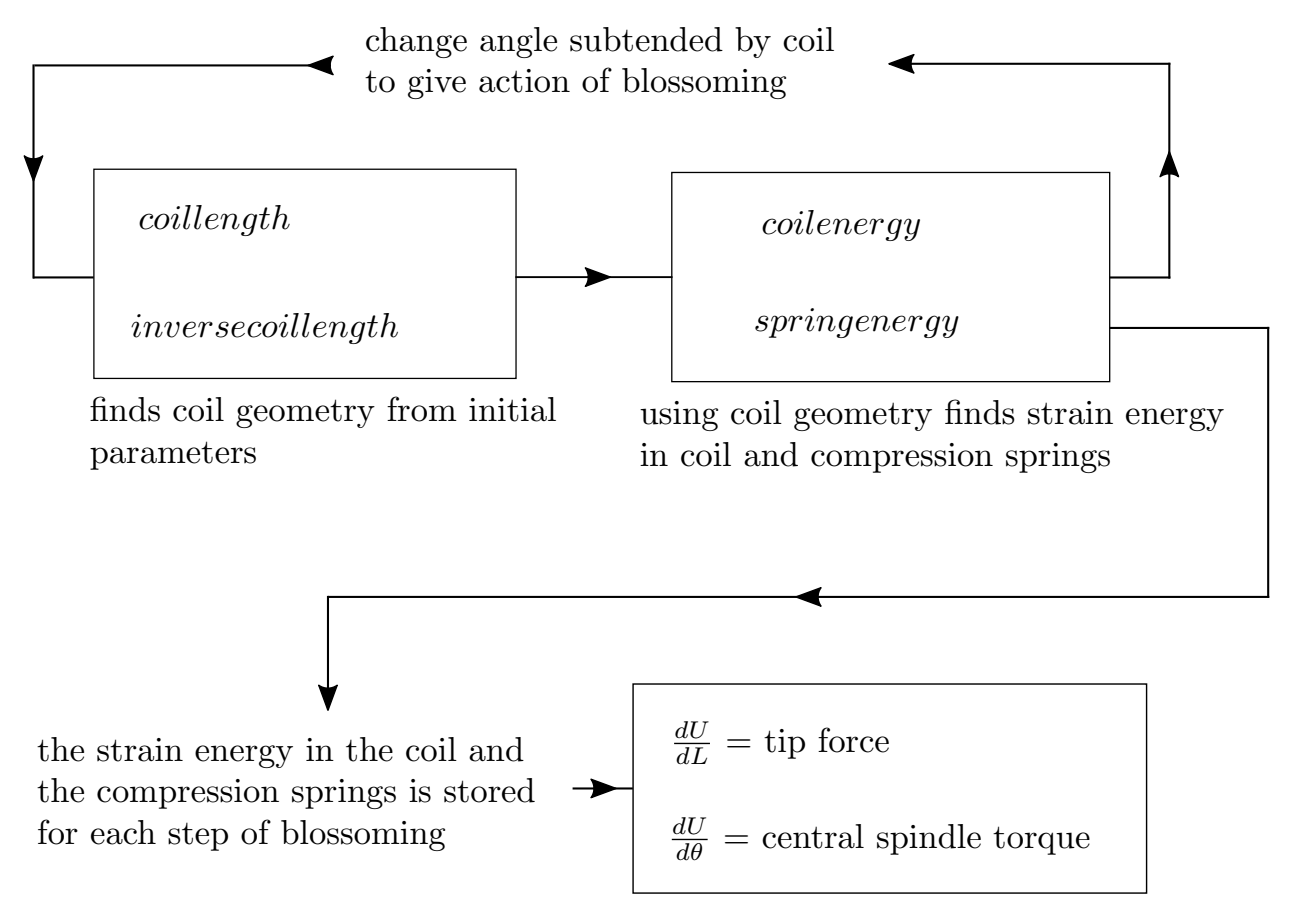

Figure 3.22: A general method of finding the tip force and central spindle torque including spiral geometry and compression springs in caption.

A comparison between the method that approximates the coil to be a circle and the method that uses the spiral geometry is shown in Figure 3.23. When the coil is at the natural radius of the tape spring there is no force for the circle method whereas there is a force for the spiral method, this is because the spiral is being held at a larger radius than would allow for its strain energy minimum. As the inner coil radius increases the difference between the two methods becomes less pronounced.

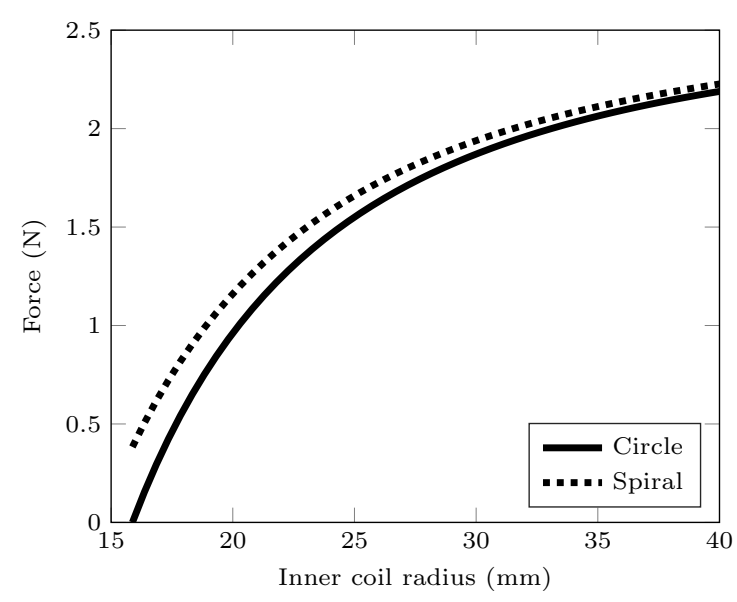

Figure 3.23: Shows a comparison between the circle and spiral method of finding the tip force at different radii. There are the 20 turns in the coil. Boom dimensions are shown in Table 3.1.

The previous figures have been plotted with a constant coil angle and a changing length to reflect the conditions in the initial experiment. A visual representation of this is shown in Figure 3.24(b). While this is an accurate representation of the first set of experiments, blossoming usually occurs when the tip stops extending but the central spindle continues to turn. In this case the length of the boom that is coiled remains constant but the number of coils decreases and the radius increases, 
as shown in Figure 3.24(c). Figure 3.25 shows a comparison between the tip force using the spiral and circle method, using a fixed tip and varying the number of turns within the coil. Initially the coil has 20 turns and the central spindle is at the spring's natural radius. As the central spindle turns but the coil doesn't extend out of the deployer the coil radius increases, this leads to an increasing force. When there are 20 turns in the coil, the difference in the force between the two methods is significant, as the coil expands and the number of coils decreases, the difference in force reduces.

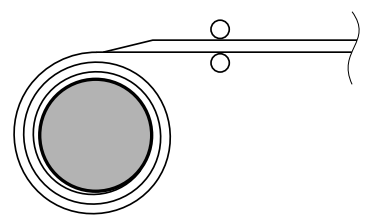

(a)

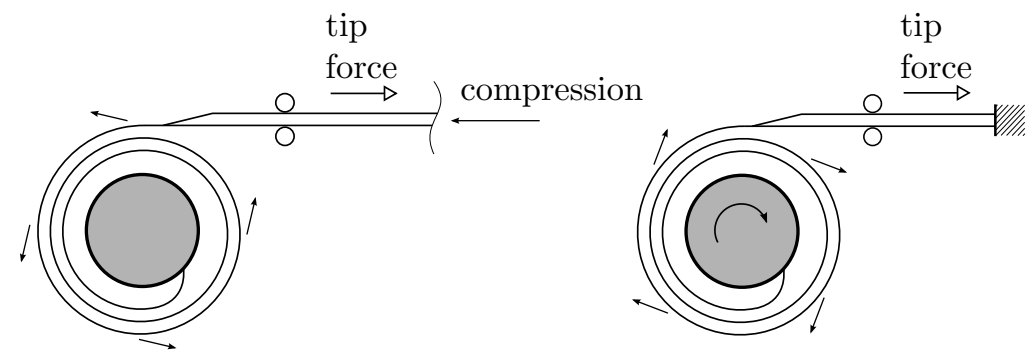

(b) (c)

Figure 3.24: (a) The initial condition of a coiled boom with no tip force. (b) The central spindle is held fixed and the boom tip is compressed towards the central spindle. The coil expands to a radius which is larger than the natural radius of the tape spring, this creates the tip force. The tip force is shown with an arrow with a large head, the rest of the arrows represent the boom motion. (c) The boom tip is held stationary, the central spindle turns and the coil unwinds. The coil radius is larger than the natural radius of the tape spring, creating the tip force. This motion is a closer representation of blossoming.

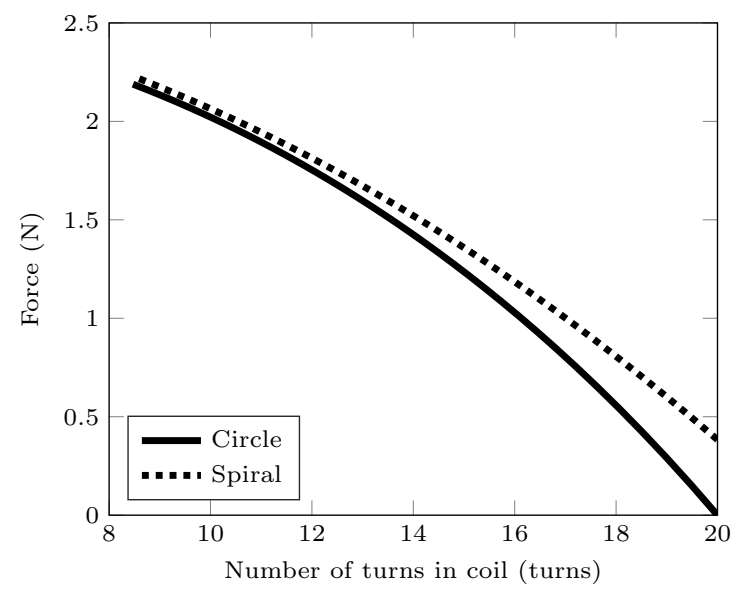

Figure 3.25: A comparison between the circle and spiral method of finding the tip force as the central spindle is turned. There are 20 turns in the coil. Boom dimensions are shown in Table 3.1 .

\subsection{Addition of compression springs}

When blossoming occurs, the outside of the coil grows in diameter, this pushes the compression rollers away from the central spindle and compresses the compression springs. The coiled boom and the compression springs can be thought of as a set of springs in parallel, as shown in Figure 3.26. As the coil expands, there is a relative motion between the amount the central spindle rotates and the change in the outside diameter of the coil, and hence the displacement of the compression rollers. This is shown in Figure 3.26 as a lever arm giving a motion ratio. In the deployer, the motion ratio will depend on the coil geometry and can be calculated in MATLAB using Equations 3.17, 3.18 and 3.19. As the compression springs are compressed by the expanding coiled boom, their stored 
strain energy will increase. In the case of a linear compression spring, the strain energy is given by Equation 3.20 where $k$ is the spring stiffness and $x$ is the spring displacement. In the case of other springs, such as torsion springs, a similar method could be used. The increase in compression spring strain energy can be added to the strain energy of the coiled boom to give an overall strain energy. By calculating the gradient of the total strain energy with respect to the tip displacement, the tip force including the effect of the compression springs can be found as blossoming occurs.

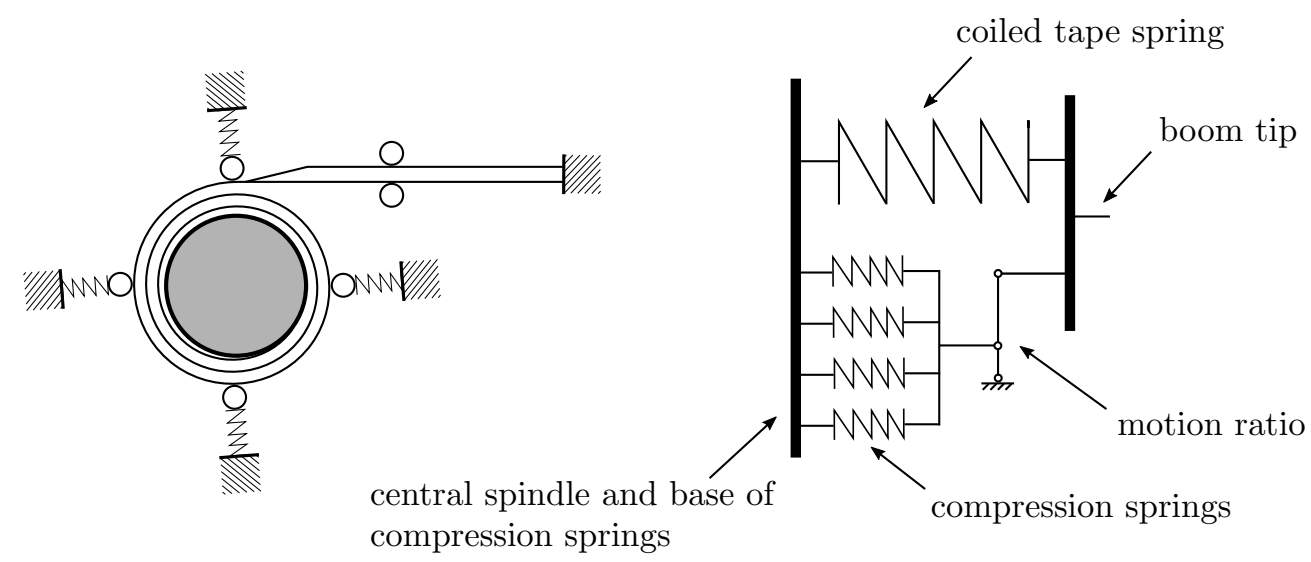

Figure 3.26: A coiled boom and compression springs can be thought of as a main spring and four compression springs in parallel with a motion ratio between the movement of the boom tip and the compression springs.

$$
U=\frac{1}{2} k x^{2}
$$

If the coiled boom has even a moderate number of turns, the compression spring displacement will be quite small for given tip displacement. Because of this small displacement, the compression springs do not create a significant increase in tip force from the increase in strain energy.

In the following figures the compression springs used are modelled as torsion springs with compression arms, as this the configuration of the experimental test set-up, as shown in Figure 3.29. The same principle is used to calculate the increase in tip force due to the torsion springs as was used for the compression springs. The change in coil diameter moves the compression rollers which turn the compression arms and hence torsion springs. The strain energy of the torsion springs is added to the strain energy stored in the coil. The gradient of the total strain energy with respect to the tip displacement gives the tip force.

Figure 3.27 shows the effect of the addition of torsion springs with no pre-tension. Initially there is no increase in force. As the central spindle turns and the coil blossoms the springs are displaced, increasing the tip force slightly. Figure 3.28 shows the effect that the springs have when they are pre-tensioned. The torsion springs have increased the tip force by approximately $0.5 \mathrm{~N}$. 


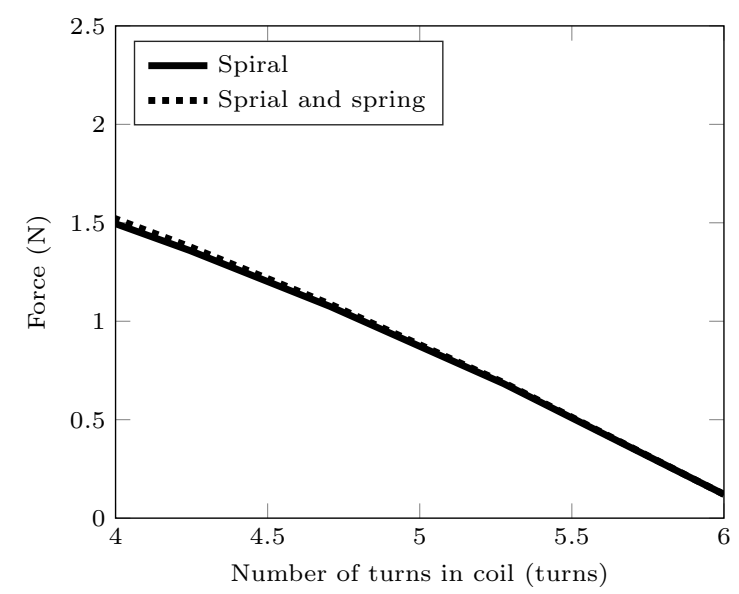

Figure 3.27: The effect that adding compression springs has on the tip force as the central spindle is turned. There are 20 turns in the coil. Boom dimensions are shown in Table 3.1. 4 compression springs with a torsional stiffness of $150 \mathrm{Nmm} / \mathrm{rad}$ and compression arm length of $88 \mathrm{~mm}$. The torsion springs are adjusted so there is initially no compression roller force on the coil.

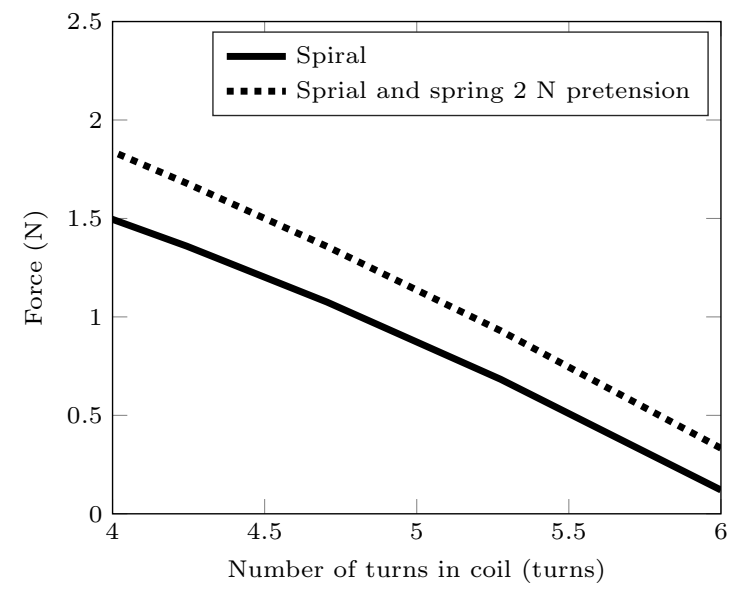

Figure 3.28: The effect that adding compression springs has on the tip force as the central spindle is turned. There are 20 turns in the coil. Boom dimensions are shown in Table 3.1. 4 compression springs with a torsional stiffness of $150 \mathrm{Nmm} / \mathrm{rad}$ and compression arm length of $88 \mathrm{~mm}$. The torsion springs are adjusted so there is initially $2 \mathrm{~N}$ of compression roller force from each of the compression rollers on the coil.

\subsection{Experimental test set-up}

To test the energy method, including the effects of the compression springs, a more elaborate experimental test set-up was created. The experimental test set-up had a central spindle whose angle could be controlled by an electric motor. The motor was attached in such a way that the torque applied by the central spindle could be measured. The four compression rollers were forced onto the coiled boom by four compression arms. The torsion springs applied a torque to the compression arms, creating the force at the compression roller. The angle of the torsion springs could be altered to give different initial forces on the boom coil. The tips of the booms were attached to the stationary force sensors. The tests consisted of adjusting the force sensors so there was no initial tip force from the booms. The torsion springs would be adjusted so that compression rollers applied the correct amount of force on the coiled booms. The central spindle was turned in increments of $36^{\circ}$ and the central spindle torque and tip forces were measured at each turn increment. By fixing the boom tips to the force sensors and turning the central spindle, 
blossoming occurred. After each experiment the booms were recoiled and the tip force reset to zero. More details of the design and operation of the experimental test set-up are given in Chapter 6 .

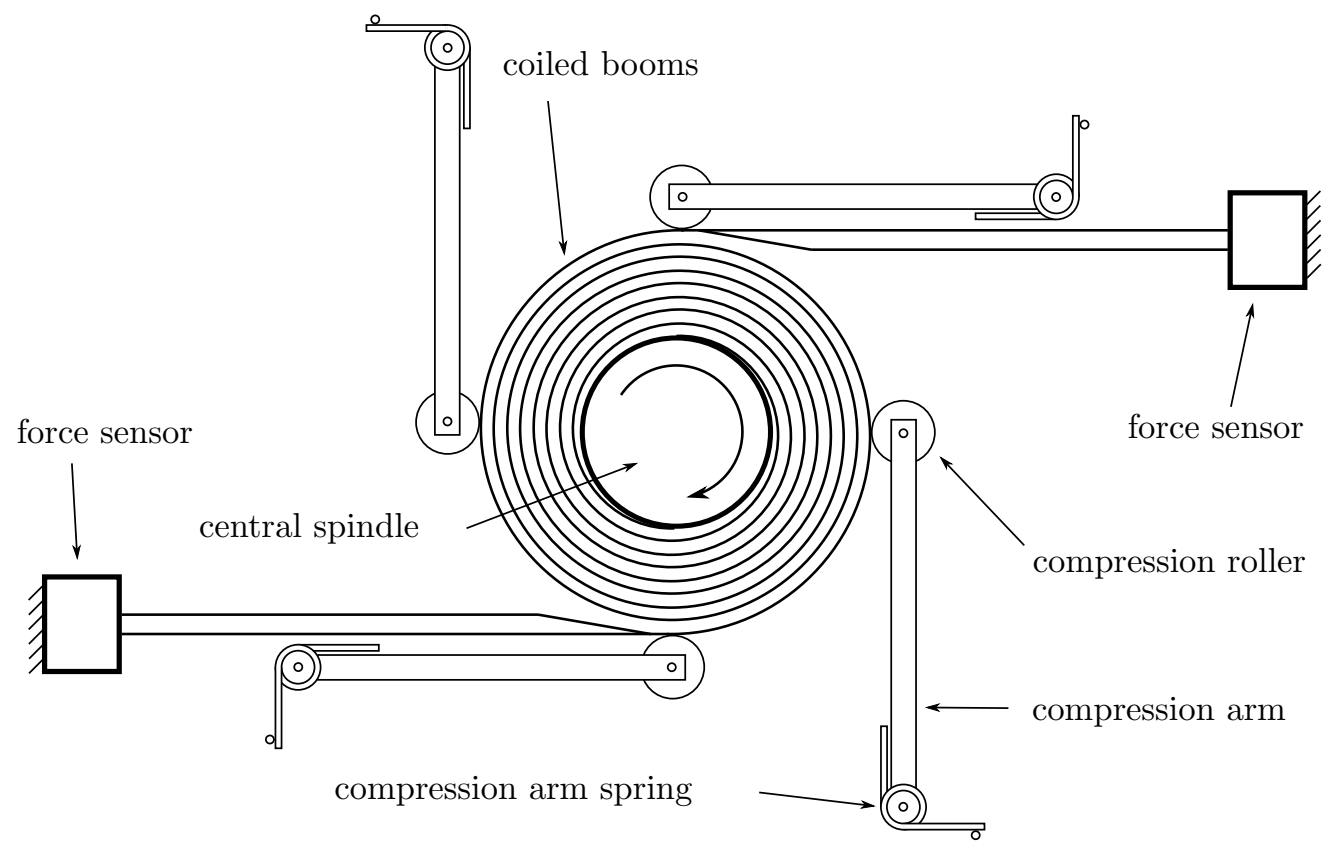

Figure 3.29: Top view of the experimental test set-up.

\subsection{Results}

To validate the strain energy method theory, tests were carried out while varying a number of different parameters. Two different booms sizes were used as well as tests with one and two booms. The number of turns in the coil and the compression roller force were also changed. For each set of parameters, three runs of the test were carried out. This is shown in the results as experimental results 1, experimental results 2 and experimental results 3. Table 3.1 shows the properties of the two booms used. Boom type A was used to carry out tests with a single boom and two booms. Boom type B was used just to carry out tests with two booms. Due to the length of boom type A, only tests with five turns could be carried out. Boom type B was longer so tests could be carried out with up to twenty turns. In all cases the the compression springs had a torsional stiffness of $150 \mathrm{Nmm} / \mathrm{rad}$ and the compression arm length between the point of rotation and where the compression roller contacted the boom coil was $88 \mathrm{~mm}$.

Using a single boom did cause a slight issue. As the boom blossomed and the coil expanded, a gap formed between the coil and the central spindle. The single boom attachment to the central spindle caused an asymmetric force to the boom coil causing it to become non-concentric with the spindle. This can be seen in Figure 3.30. This caused a slight sinusoidal increase and decrease in tip force compared to the expected force, as shown in Figure 3.32. This effect was not seen in the tests with two booms as the force from the boom attachment to the central spindle during blossoming was offset by an equal force from the other boom which was attached at $180^{\circ}$ to the first boom. This can be seen in Figure 3.31. As the coiled boom blossomed and expanded the boom coil stayed concentric to the central spindle.

Figures 3.30 and 3.31 also illustrate another point. The number of coils has an effect on how quickly the coil radius will change during blossoming. Both experiments turned the central spindle the same amount but the case with five coils shows the coil radius increasing significantly more than the case with twenty coils. 
Table 3.2: Table of properties for the booms used in the following experiments.

\begin{tabular}{|l||l|l|l|l|l|l|l|}
\hline Boom & $\mathrm{b}(\mathrm{mm})$ & $\mathrm{t}(\mathrm{mm})$ & $\mathrm{r}_{\mathrm{y}}(\mathrm{mm})$ & $\mathrm{E}(\mathrm{GPa})$ & $\nu$ & Number of turns & Spindle radius $(\mathrm{mm})$ \\
\hline \hline $\mathrm{A}$ & 31.8 & 0.13 & 12.5 & 205 & 0.3 & 5 & 15 \\
\hline $\mathrm{B}$ & 25.4 & 0.13 & 17.5 & 205 & 0.3 & $5,10,15$ and 20 & 20 \\
\hline
\end{tabular}

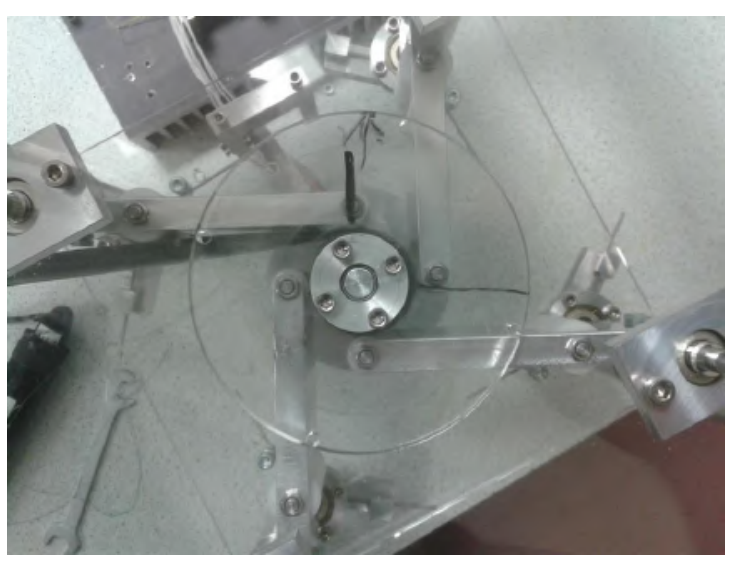

(a) 0 turns of blossoming.

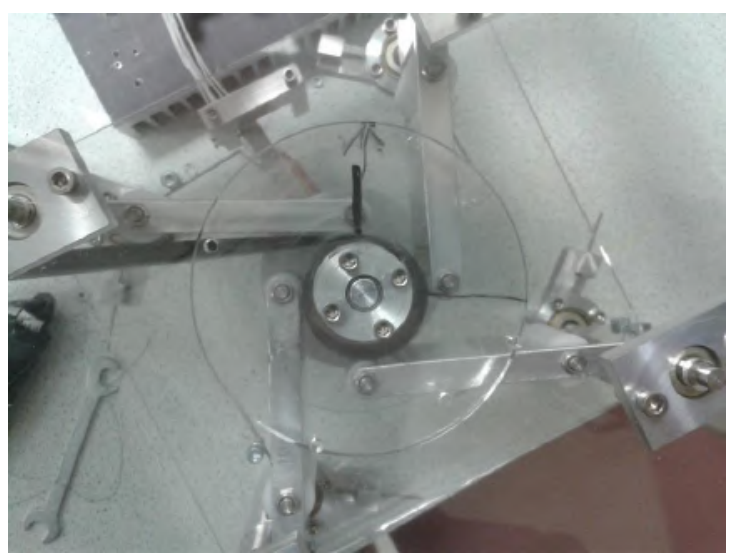

(c) 1 turn of blossoming.

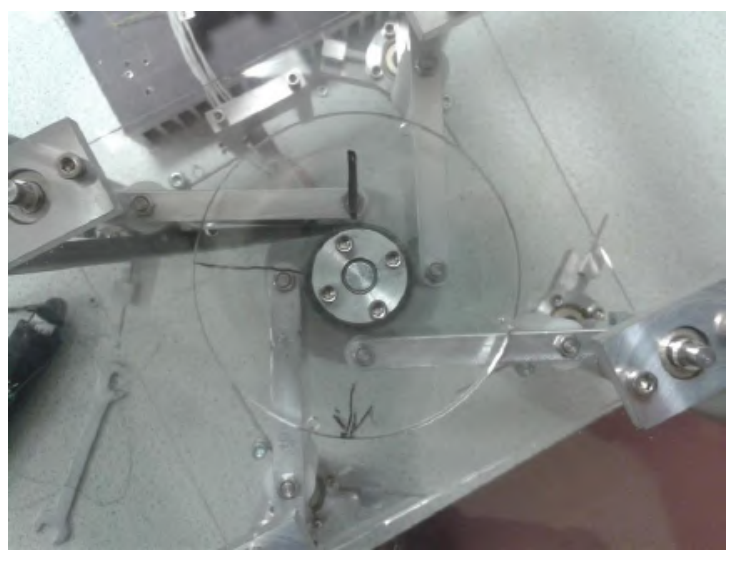

(b) 0.5 turn of blossoming.

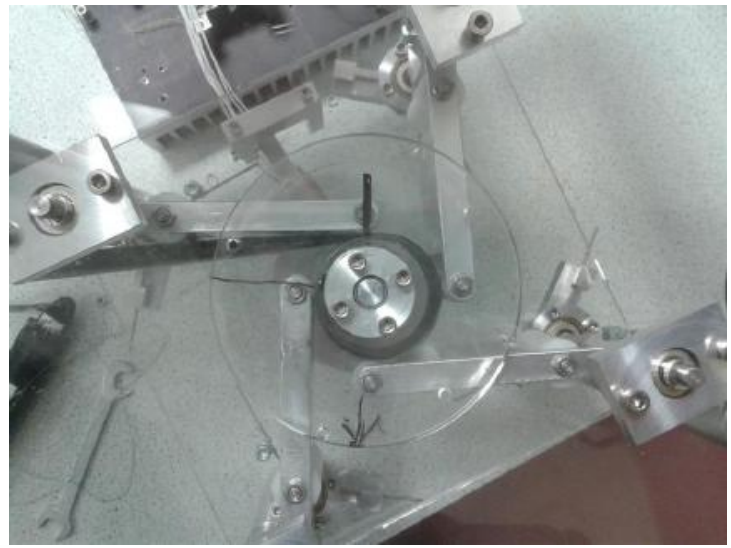

(d) 1.5 turns of blossoming.

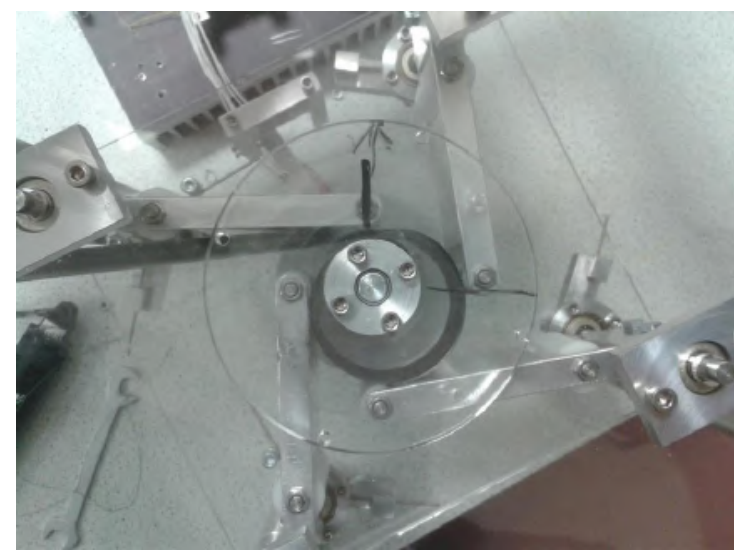

(e) 2 turns of blossoming.

Figure 3.30: A single boom with 5 turns during blossoming, using boom type A. The coil has become non concentric with the central spindle. The coil has increased significantly in diameter due to the small number of coils. 


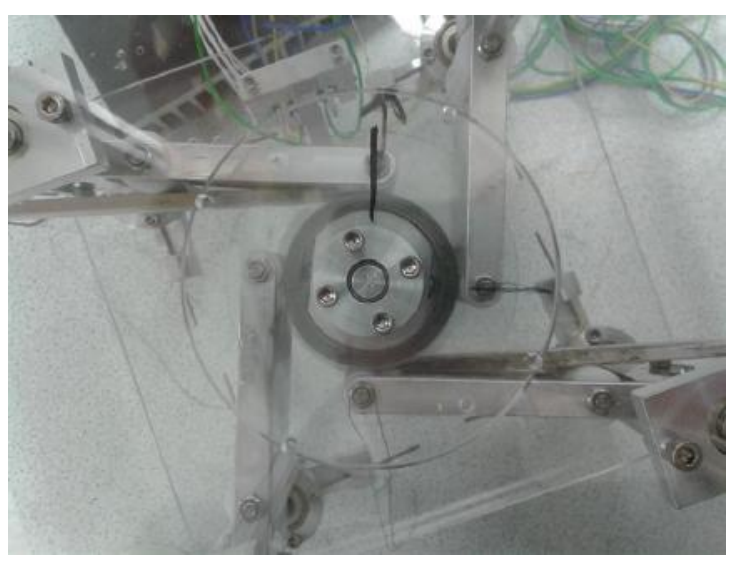

(a) 0 turns of blossoming.

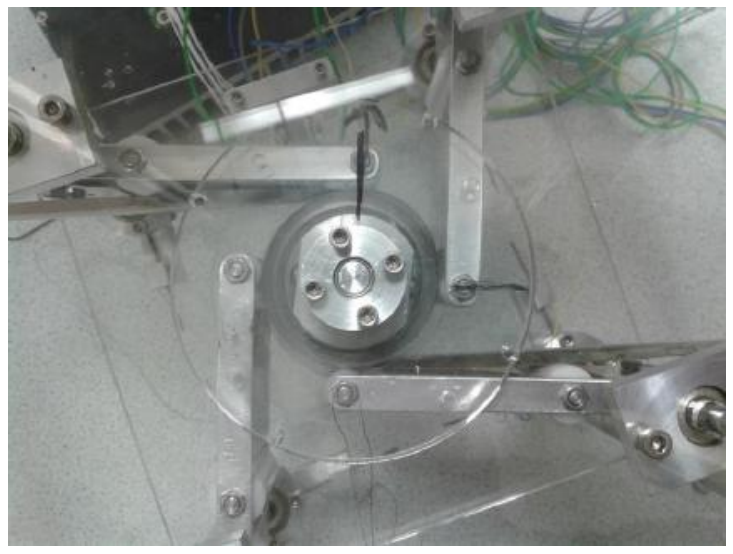

(c) 1 turn of blossoming.

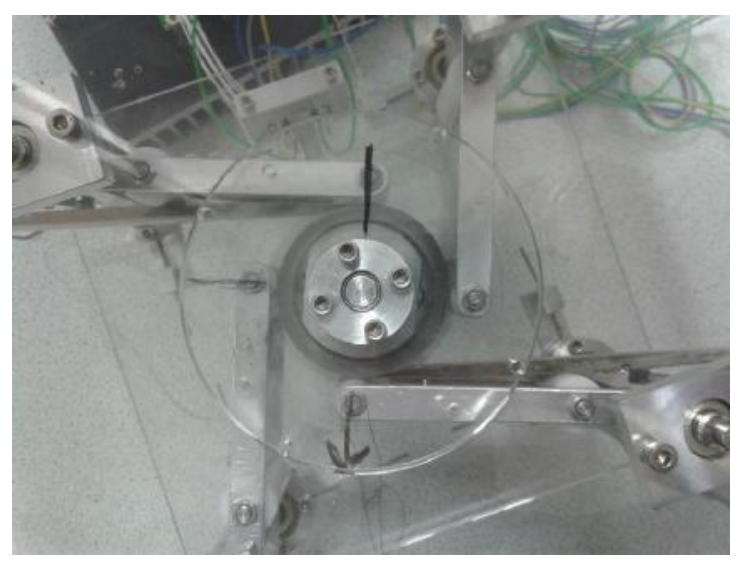

(b) 0.5 turns of blossoming.

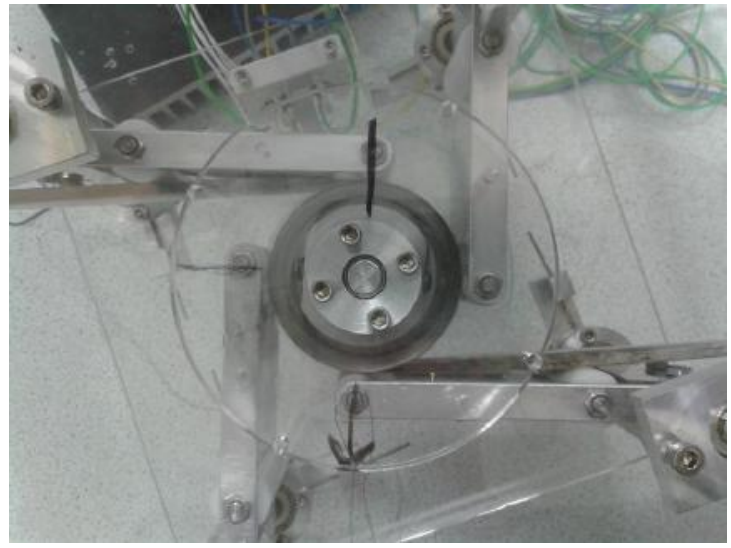

(d) 1.5 turns of blossoming.

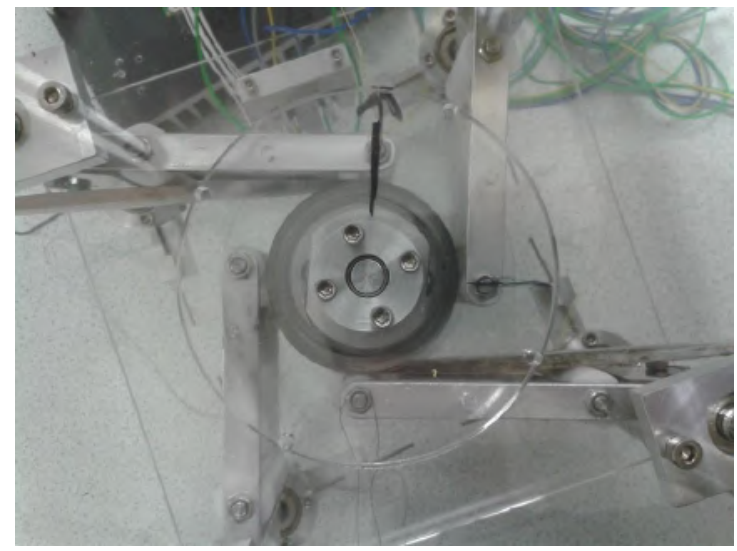

(e) 2 turns of blossoming.

Figure 3.31: Two booms with 20 coils during blossoming, using boom type B. The boom coil has stayed concentric to the central spindle. The coil has not increased significantly in diameter due to the large number of coils.

Figure 3.32 shows the theoretical and practical results for the tests with a single boom, using boom type A with five turns in the coil. The theoretical results for these tests show quite a steep increase in tip force as blossoming occurred. In Figure 3.32(a) the tip force is initially approximately $1.5 \mathrm{~N}$. As the central spindle turns and the number of coils decreases, the coil radius increases, this causes the tip force to increase to over $2.5 \mathrm{~N}$. The practical results show a sinusoidal increase and decrease in tip force compared to the force predicted by the theory. The coil becoming non-concentric to the central spindle was found to be the cause of this. While this had some effect on the practical results, the general trend seems to match the theory for the test with $1 \mathrm{~N}$ compression roller forces. As the compression roller force was increased, the practical results matched the gradient of tip force 
during blossoming, but the level of force seemed to increase above that predicted by the theory, as shown in Figures 3.32(b-d). The increase in practical force as the compression roller force is increased is thought to be due to the greater friction force between the coil layers. For blossoming to occur, the layers of the coil must slide past one another. By increasing the compression roller force, the compression between each layer of coil increased, making it harder for the layers to slide therefore increasing the tip force.

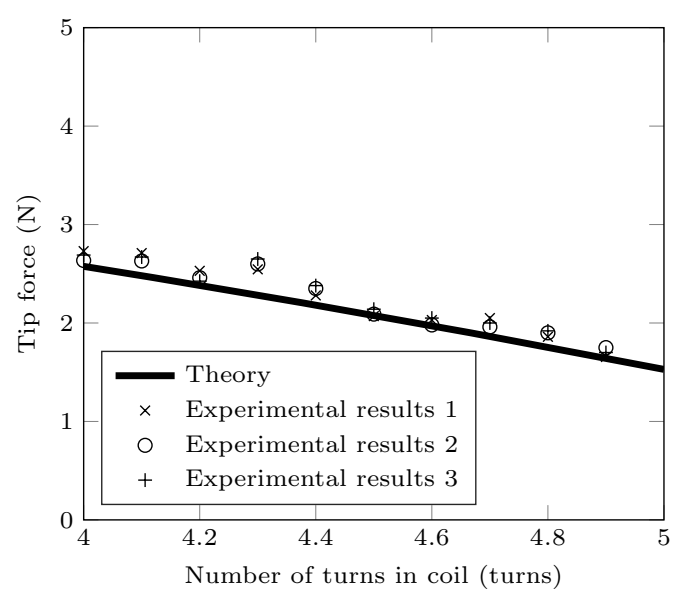

(a) $1 \mathrm{~N}$ compression spring force.

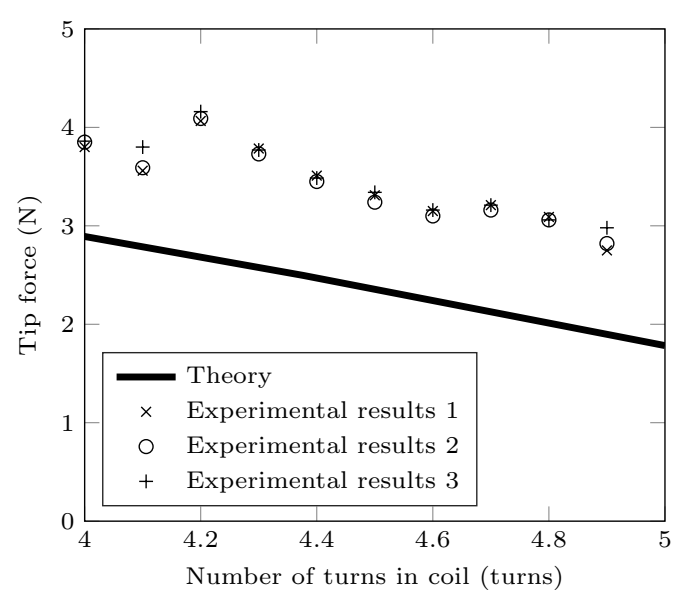

(c) $3 \mathrm{~N}$ compression spring force.

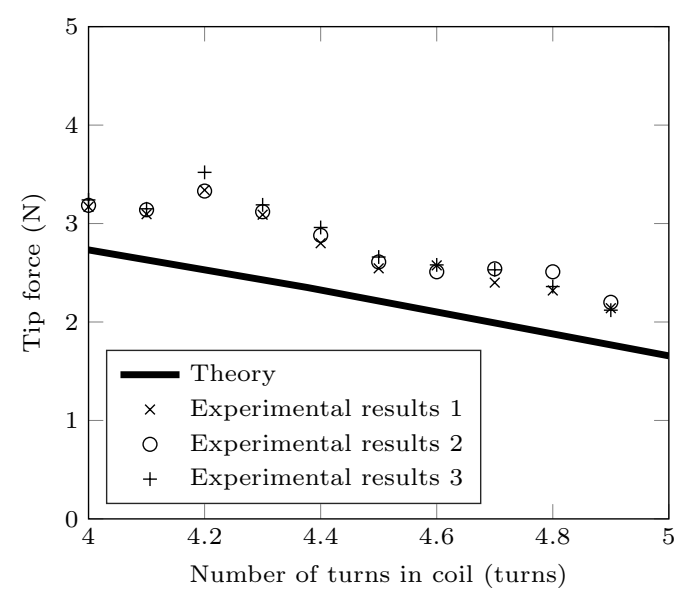

(b) $2 \mathrm{~N}$ compression spring force.

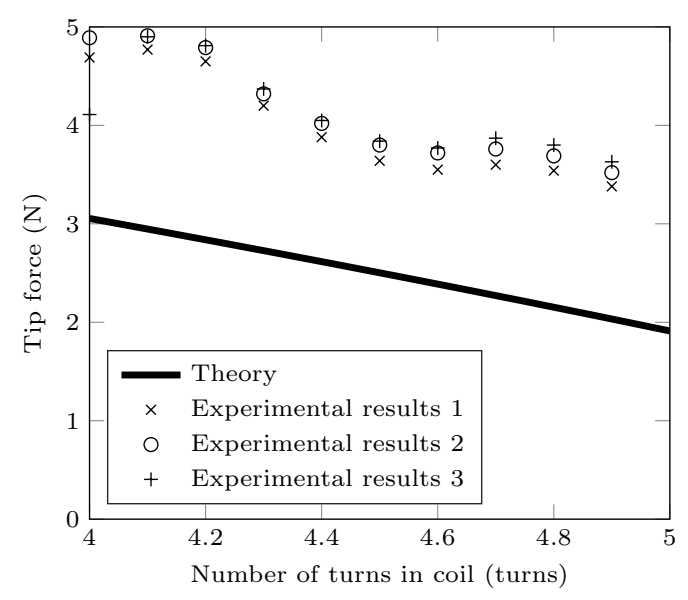

(d) $4 \mathrm{~N}$ compression spring force.

Figure 3.32: Results for boom type A, using a single boom and 5 coils.

Figure 3.33 shows the theoretical and practical results for boom type A with five coils and with two booms. The theoretical results follow closely the results for the single boom. The practical results exhibit no sinusoidal increase and decrease in tip force that was associated with the single boom and also follow the theoretical increase in tip force as blossoming occurs. The practical results follow the same pattern of quite closely matching the theoretical results in the case of a $1 \mathrm{~N}$ compression roller force but as the compression roller force is increased the practical results are significantly higher than the theory predicts. The increase in friction within the coil as the compression roller force is increased is thought to have caused this increase in tip force.

There is a larger spread of practical data compared to the results for the single boom. Before each test the central spindle is bought back to the starting position with five turns in the coil. Due to the slightly inconsistent nature of the coiling and uncoiling process the central spindle zero position does not always result in a zero tip force. To allow for this, the force sensors have a small amount of displacement adjustment so their position can be adjusted, allowing the next test to start with zero tip force. The adjustment of the force sensor may have caused one boom tip to be slightly offset compared to the other boom causing the coil to be not exactly concentric to the 
central spindle. This may have caused the increased scatter in the data. The data seems to suggest that as one boom creates more force the other boom produces less. Although there is a significant amount of scatter, the general trend still seems to follow the same pattern as for the cases with the single boom.

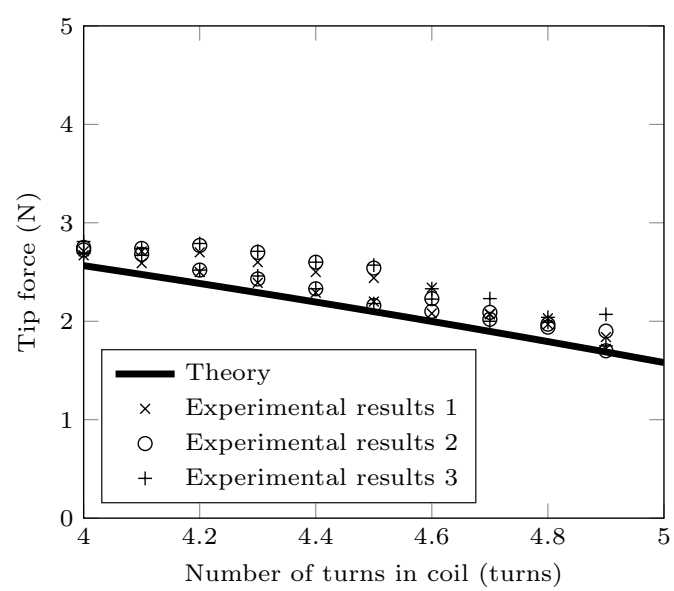

(a) $1 \mathrm{~N}$ compression spring force.

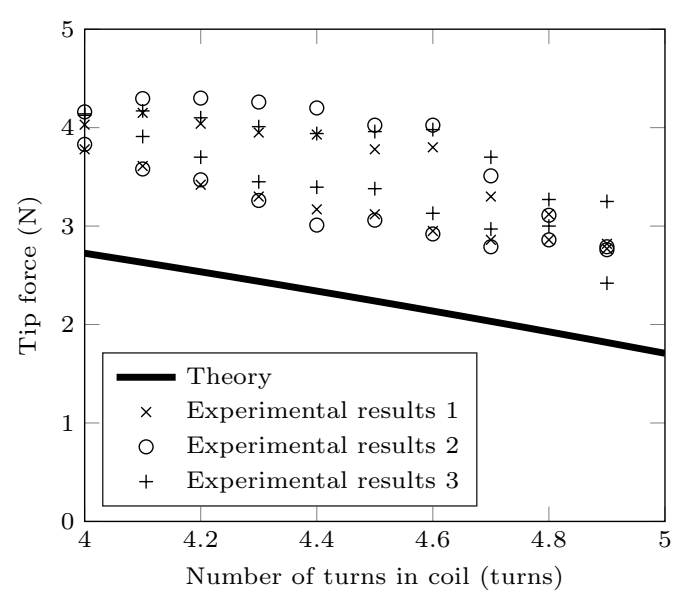

(c) $3 \mathrm{~N}$ compression spring force.

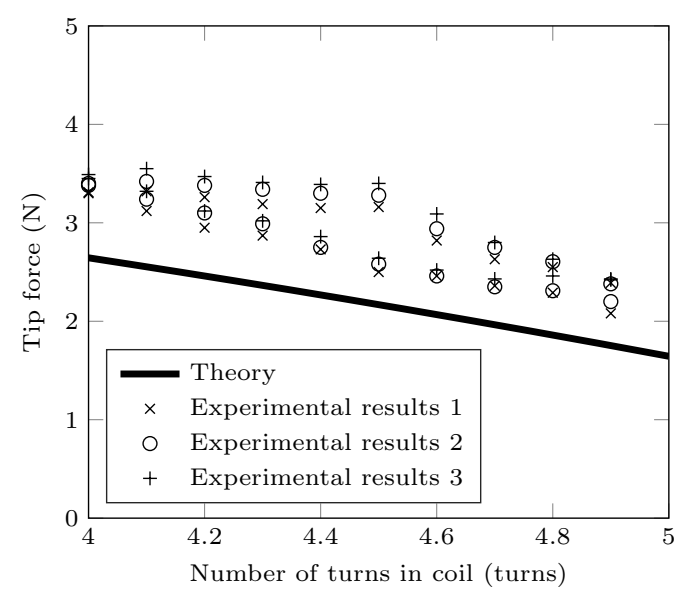

(b) $2 \mathrm{~N}$ compression spring force.

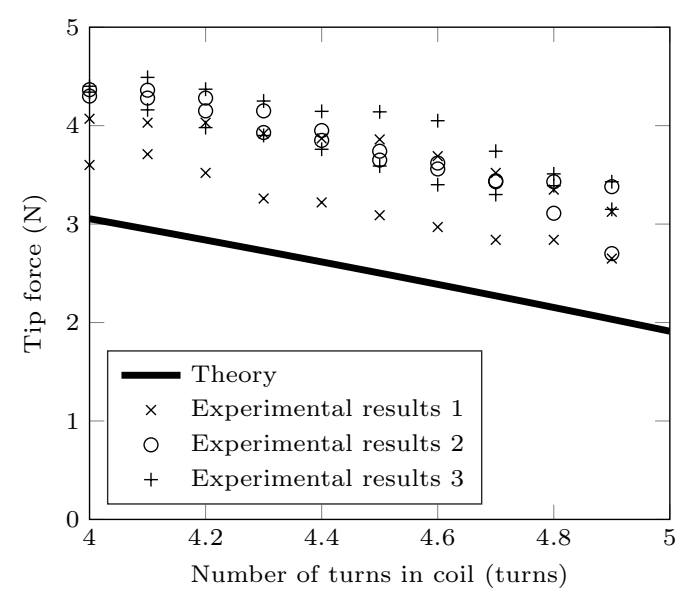

(d) $4 \mathrm{~N}$ compression spring force.

Figure 3.33: Results for boom type A, using two booms and 5 coils.

Figures 3.34 show the results for tests with boom type B, with five coils, using two booms. The theoretical results show significantly less tip force than for boom type A, this is due to the smaller width and larger natural radius of boom type B, meaning less strain energy within the coil. The tip force increases during blossoming with a similar gradient to the cases with boom type A. The practical results correlate quite well with the theoretical predictions, showing the same increase in tip force as blossoming occurs. The practical tip force increases above the theoretical prediction as the compression roller force is increased in a similar manner to the tests with the boom type A. This is thought to be due to the increase in friction within the boom coil. 


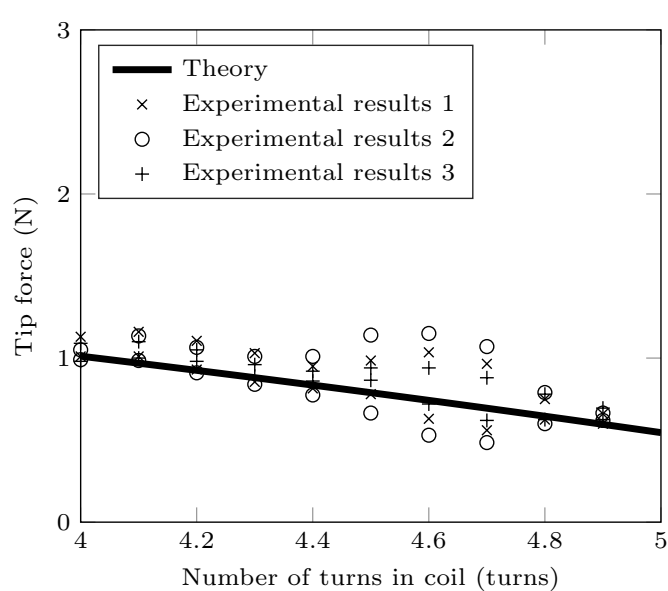

(a) $1 \mathrm{~N}$ compression spring force.

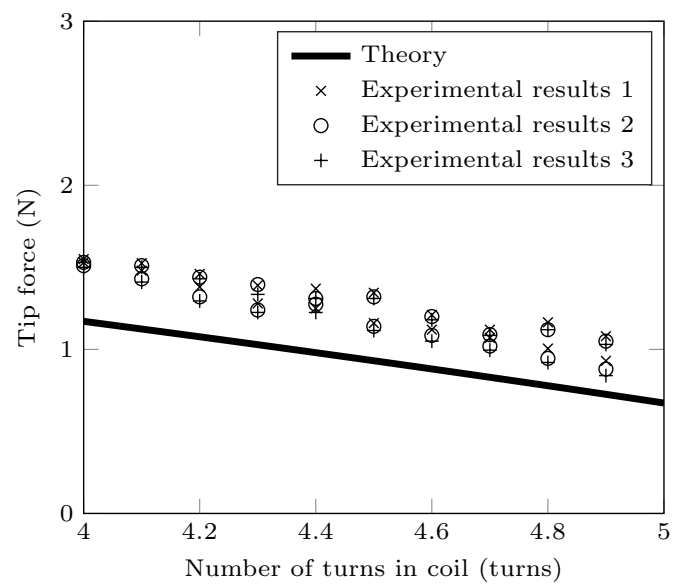

(c) $3 \mathrm{~N}$ compression spring force.

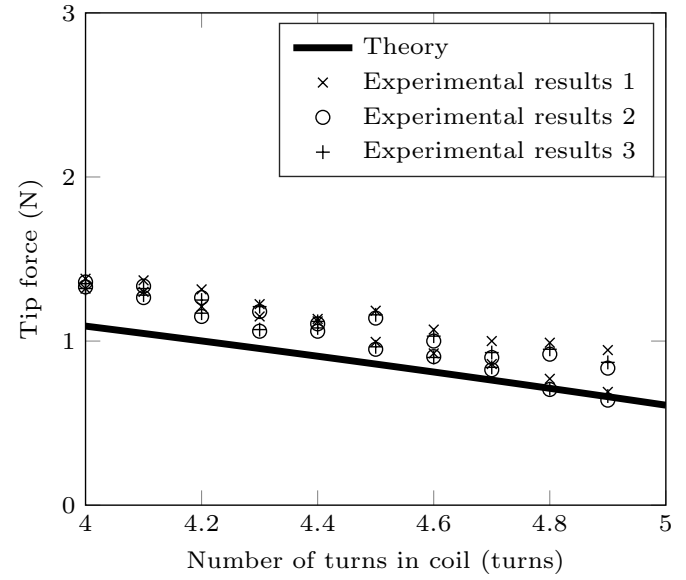

(b) $2 \mathrm{~N}$ compression spring force.

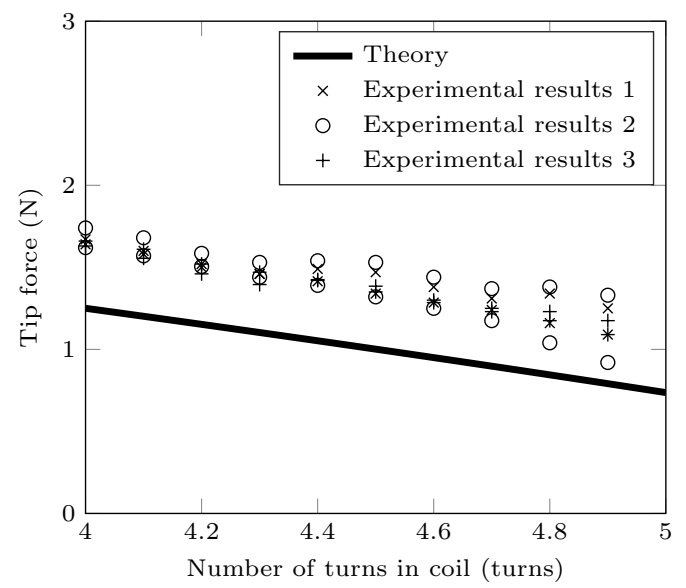

(d) $4 \mathrm{~N}$ compression spring force.

Figure 3.34: Results for boom type B, using two booms and 5 coils.

Figure 3.35 shows the results for tests using boom type B, with ten turns in the coil, using two booms. The theoretical results have less of an increase in force as blossoming occurs compared to the cases with 5 turns in the coil. The greater number of coils leads to less of an increase in coil radius for each turn blossomed, this leads to the smaller increase in tip force. The practical results show a similar trend of being quite close to the theoretical prediction for the case with 1 $\mathrm{N}$ of compression roller force but the practical results increase above the theoretical predictions as the compression roller force is increased. 


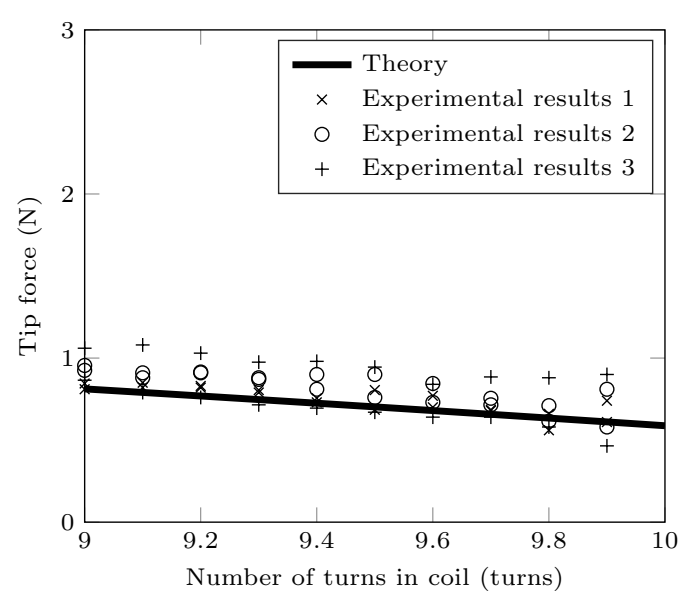

(a) $1 \mathrm{~N}$ compression spring force.

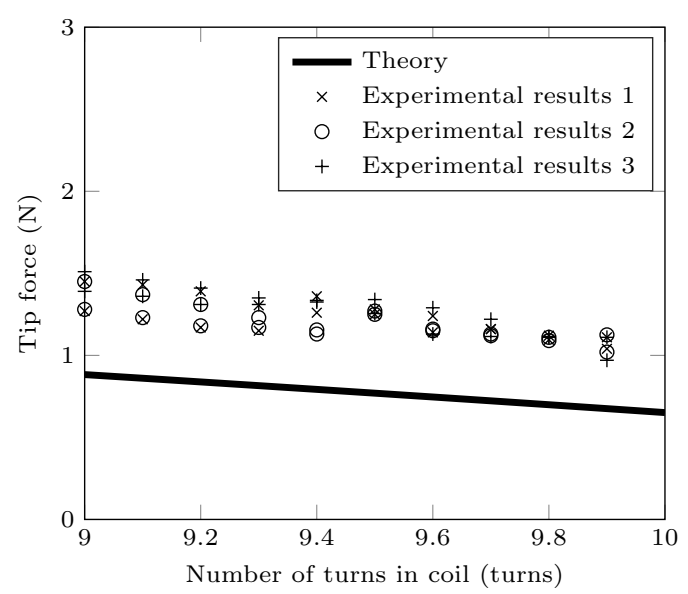

(c) $3 \mathrm{~N}$ compression spring force.

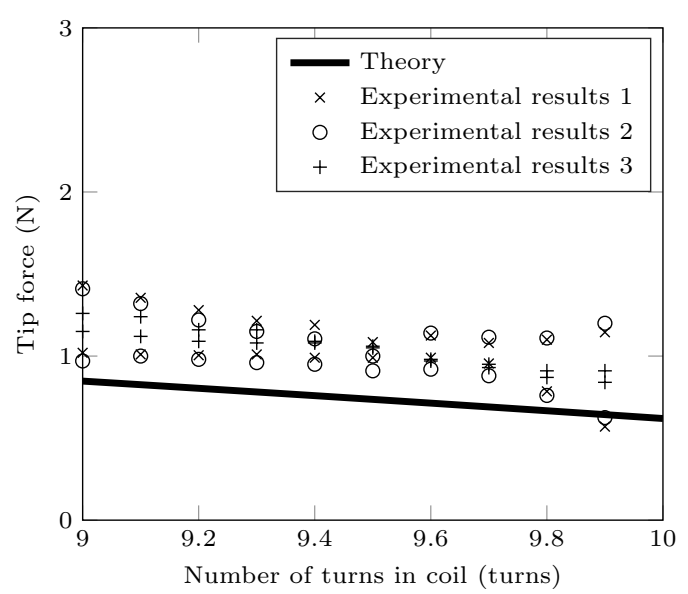

(b) $2 \mathrm{~N}$ compression spring force.

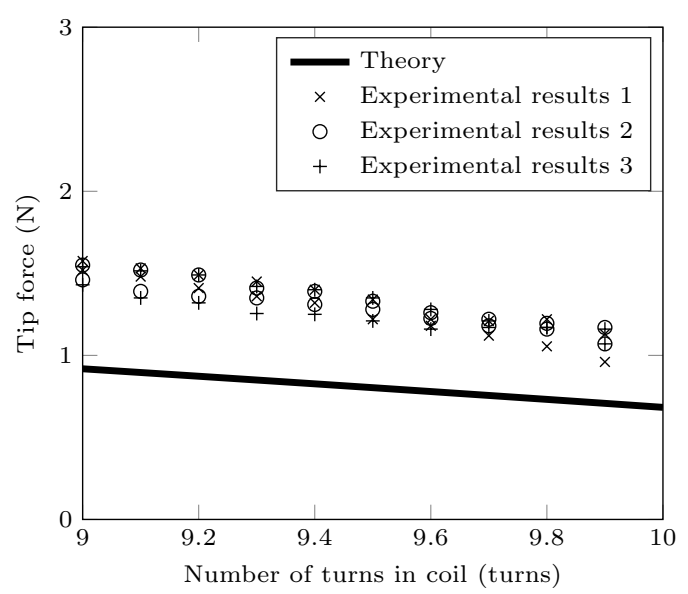

(d) $4 \mathrm{~N}$ compression spring force.

Figure 3.35: Results for boom type B, using two booms and 10 coils.

Figures 3.36 and 3.37 show the results for boom type B, using fifteen and twenty coils, with two booms. The same general trends as shown in the previous figures can be seen. The higher number of coils leads to a smaller increase in coil radius for one turn of blossoming, this causes a smaller increase in tip force compared to the cases with fewer coils. In the cases with $1 \mathrm{~N}$ of compression roller force the practical results are similar to the theoretical predictions, as the compression roller force is increased the practical tip force increases above the theoretical predictions. 


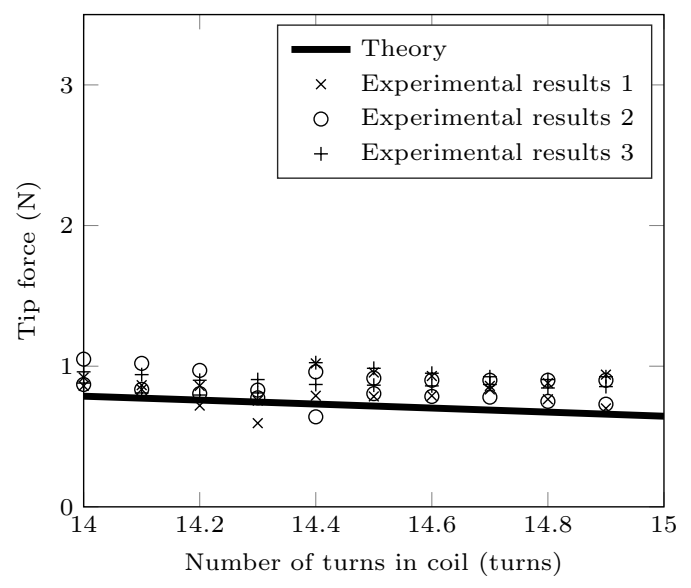

(a) $1 \mathrm{~N}$ compression spring force.

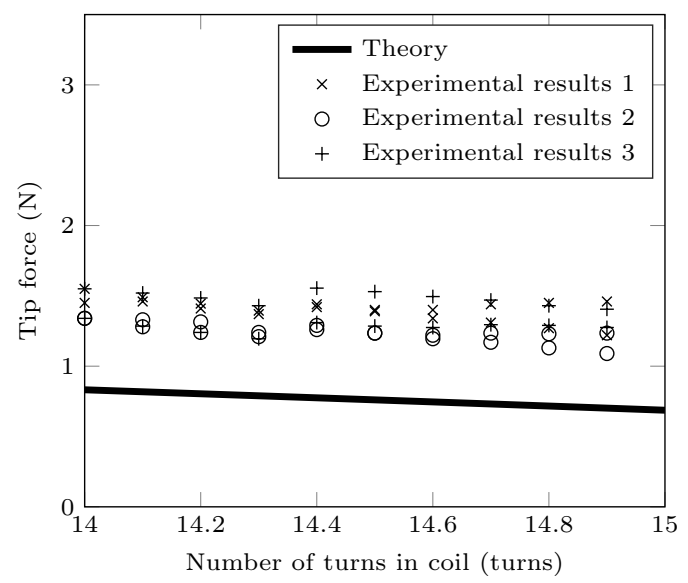

(c) $3 \mathrm{~N}$ compression spring force.

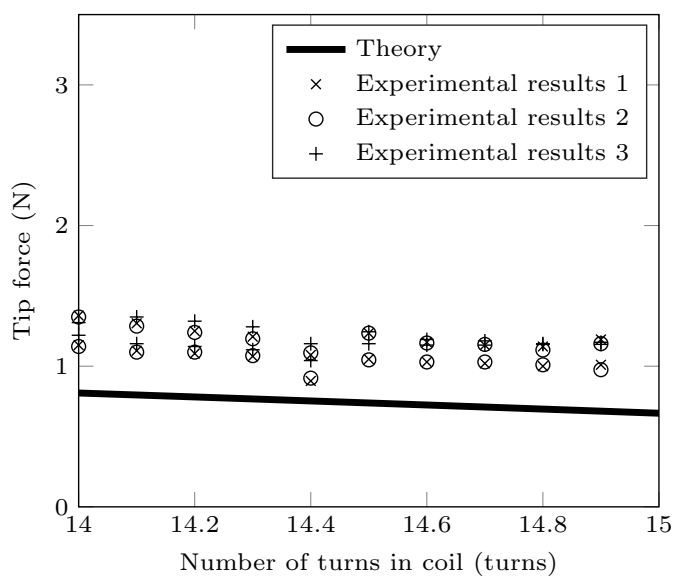

(b) $2 \mathrm{~N}$ compression spring force.

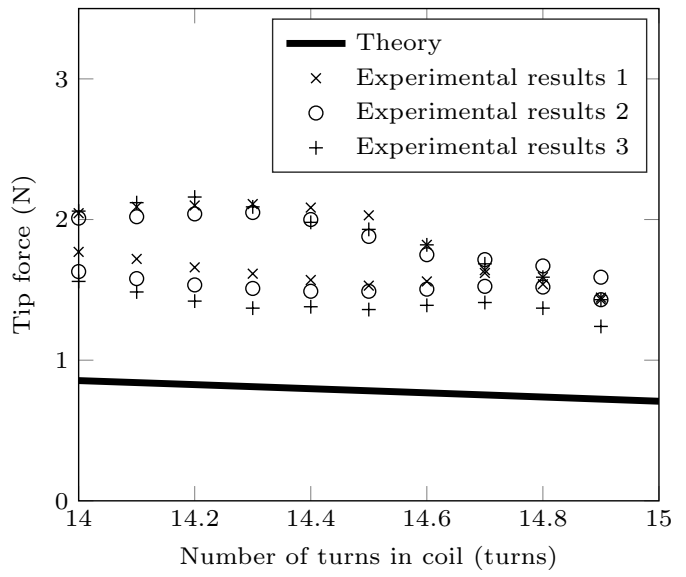

(d) $4 \mathrm{~N}$ compression spring force.

Figure 3.36: Results for boom type B, using two booms and 15 coils. 


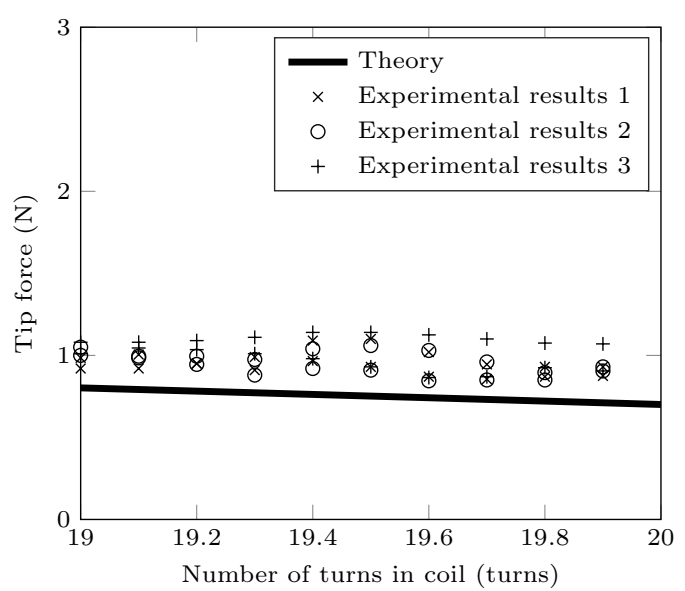

(a) $1 \mathrm{~N}$ compression spring force.

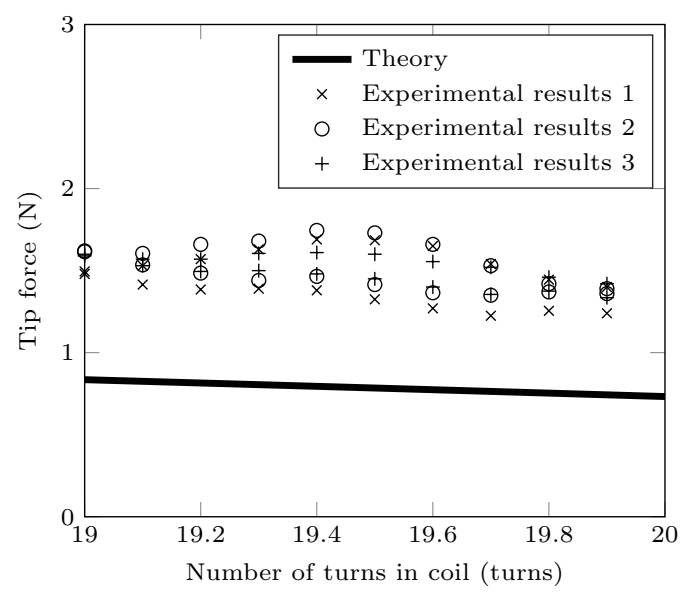

(c) $3 \mathrm{~N}$ compression spring force.

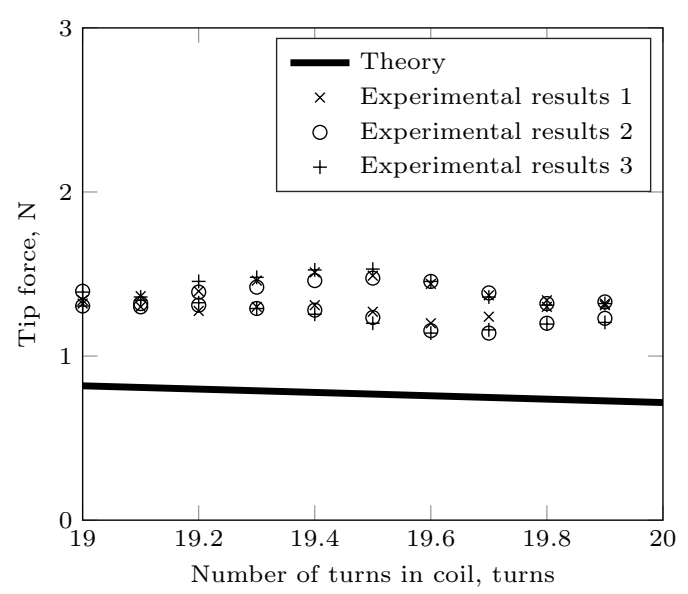

(b) $2 \mathrm{~N}$ compression spring force.

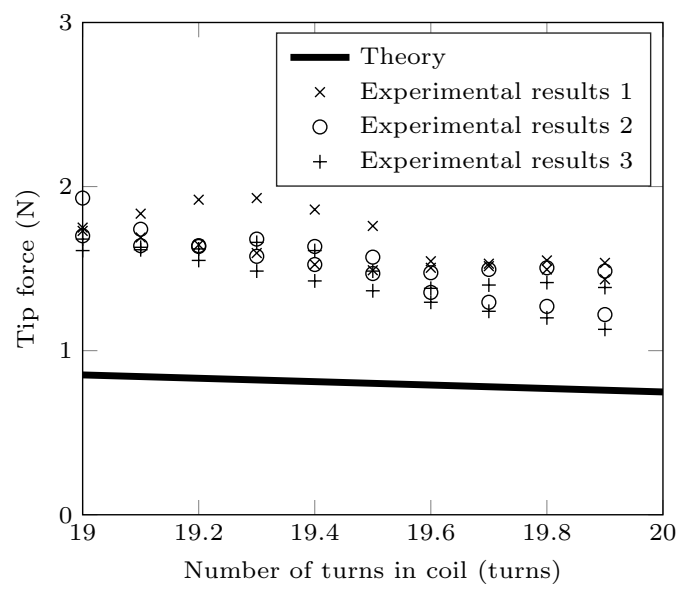

(d) $4 \mathrm{~N}$ compression spring force.

Figure 3.37: Results for boom type B, using two booms and 20 coils.

\subsection{Energy method conclusion}

A method using the strain energy stored in a coiled boom has been developed to predict the tip force during blossoming. The method has been developed to include the effects of the compression roller force and to take in to account the spiral geometry of the coil. To validate the theory, tests were carried out using a specially designed experimental test set-up.

The theoretical results show some general trends. The initial tip force for all the cases was above zero, this was because the central spindle radius was larger than the tape spring natural radius. The strain energy from the compression rollers also helped this effect. The cases with boom type A show larger forces than for the cases with boom type B, this was due to boom type B having a smaller width and having a larger natural radius. In the cases with a small number of turns in the coil, the tip force increased significantly as blossoming occurred. This was due to a significant increase in coil radius. In cases with a larger number of coils, the coil radius did not increase as much, this lead to a smaller increase in tip force during blossoming. In all the cases a very small increase in tip force could be seen as the compression roller force was increased. The increase in force was small due to the large motion ratio between the movement of the compression springs and the movement of the boom tip. The effectiveness of the compression rollers decreases further as the number of turns in the coil increases. In the cases with five turns in the coil, the change in tip force from $1 \mathrm{~N}$ of compression roller force to $4 \mathrm{~N}$ of compression roller force was approximately $0.25 \mathrm{~N}$. In the cases with twenty turns in the coil the increase in tip force was less than $0.1 \mathrm{~N}$.

The practical results showed quite good correlation to the theoretical predictions in the cases with 
$1 \mathrm{~N}$ of compression roller force. In all cases the practical tip force matched the theoretical increase in tip force during blossoming. In the cases with a small number of turns in the coil and therefore a large increase in tip force, the practical results also displayed a large increase in tip force. In the cases with a large number of turns in the coil and therefore a small increase in tip force, the practical results also displayed a small increase in tip force during blossoming. In some cases there was a significant amount of scatter due to possible asymmetry in the position of the boom tips and hence the non-concentricity of the coiled boom and the central spindle, or possibly due to the inconsistent nature of the coiling and uncoiling of the booms. While the gradient of the tip force during blossoming seemed to correlate quite well to the theoretical predictions, the magnitude of the tip force seemed to increase significantly above the predictions as the compression roller force was increased. The increase in tip force as the compression roller force was increased is thought to be due to the increase in friction within the coil. For blossoming to occur each layer of coiled boom has to slide past the next layer. The compression rollers apply a force to the outside of the coil, this is transmitted through each layer. Increasing the compression roller force increases the force between each layer of coiled boom making it harder for sliding to occur and therefore increasing the tip force.

The energy method is a good starting point in predicting the tip force during blossoming although it is obvious that friction within the boom coil adds an extra force which is dependant on the compression roller force. A model for the friction force will have to be taken in to account to more accurately predict the tip force. 


\section{Chapter 4}

\section{Central spindle and self-deployment torque}

This chapter develops a strain energy method to find the central spindle torque and the selfdeployment torque during blossoming. The part of the boom that is on the outside of the coil changes from a stressed state when coiled to an unstressed state once deployed, this leads to the self-deployment torque. The central spindle torque originates from the coil trying to obtain a lower strain energy state by uncoiling from the inside of the boom coil where it attaches to the central spindle. The effect that the tip force has on the central spindle torque is also investigated. Initially the radius from the inside to the outside of the coil is taken as constant to simplify the analysis. Finally the spiral geometry that was developed in the previous chapter is used to take into account the change in radius from the inside to the outside of the coil.

\subsection{Torque and force balance}

As described in Chapter 3, to form a bend in a tape spring, moments must be applied at either end. A large moment is needed to force the tape spring to snap through, and once the fold is formed, a smaller moment is needed to maintain the bend angle. When the bend angle is increased so that the tape spring forms a coil, the moments must be maintained at each end of the boom. If the moment on the inside end of the tape spring is released the coil will unwind. If the moment on the other end of the deployed part of the boom is released the outside of the coil will also unwind. In the case of a deployer, the moment at one end of the tape spring is applied by the attachment to the central spindle and the other end is prevented from uncoiling by the guide rollers, this is shown in Figure 4.1. The solid arrows represent the torques applied by the tape spring and also the tip force. The dotted arrows represent the reaction torque and forces applied to the tape spring. The central spindle reaction torque $\left(T_{c s r}\right)$ is applied to the central spindle, the compression roller force is applied by the guide roller and the sail tension is applied to the boom tip. The self-deployment torque $\left(T_{s d}\right)$ is opposed by the force from the guide roller. The central spindle torque $\left(T_{c s}\right)$ is opposed by central spindle reaction torque $\left(T_{c s r}\right)$ applied to the central spindle by the central spindle electric motor. The tip force is equal and opposite to the sail tension. When there is no sail tension and hence no tip force, the central spindle torque and the self-deployment torque are equal and opposite to one another. 


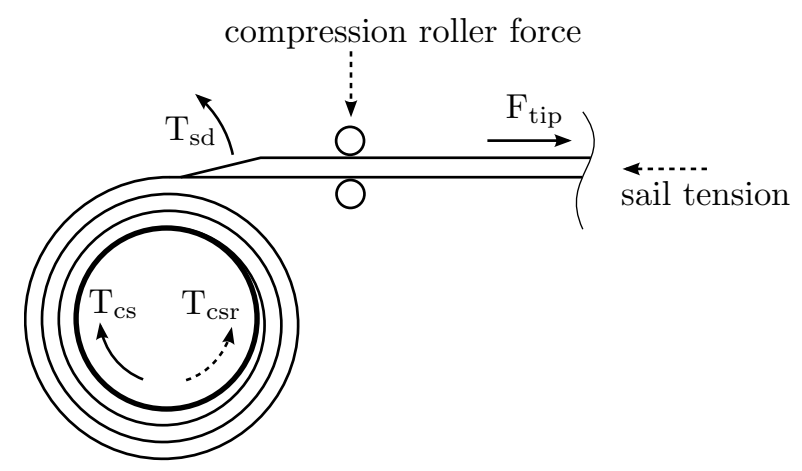

Figure 4.1: The balance of forces and torques for a coiled boom in a deployer. The solid arrows are the torques and force applied by the tape spring and the dotted arrows are the forces and torque applied to the tape spring.

The strain energy stored in the coil can be used to find the self-deployment torque and the central spindle torque. The self-deployment torque originates from the reduction in strain energy when the tape spring changes from a coiled state to a deployed state which occurs on the outside of the coil. In this situation the radius of the tape spring stays the same, but the length and angle subtended by the coil decreases. The motion that creates the self-deployment torque is shown in Figure 4.2 .
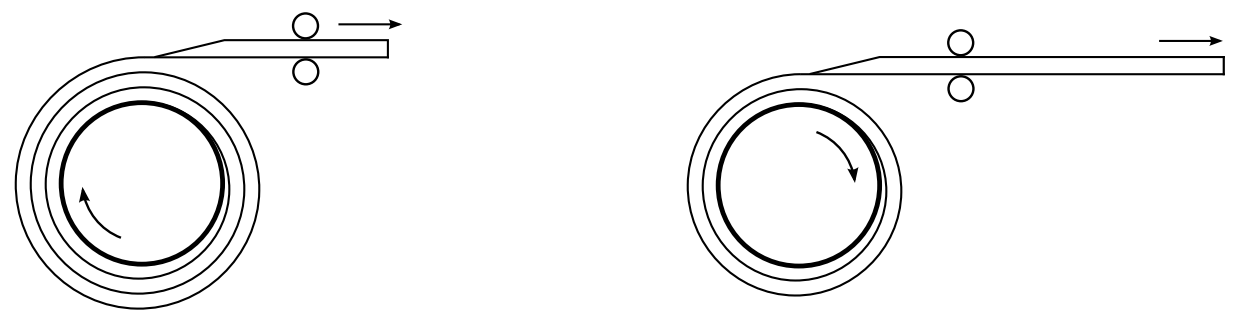

initial state

Figure 4.2: The strain energy reduction in the coil for a certain angle turned when the boom coil undergoes this motion gives rise to the self deployment torque. The coil radius remains constant but the length and angle subtended by the coil decreases.

The central spindle torque originates from the change in radius of the coil, as shown in Figure 4.3. If the boom tip is held stationary and the central spindle is allowed to turn, the coil will tend to increase in diameter as this lowers its strain energy. In this case, the length of the coiled boom stays constant but the radius of the coil increases and the angle subtended decreases. 


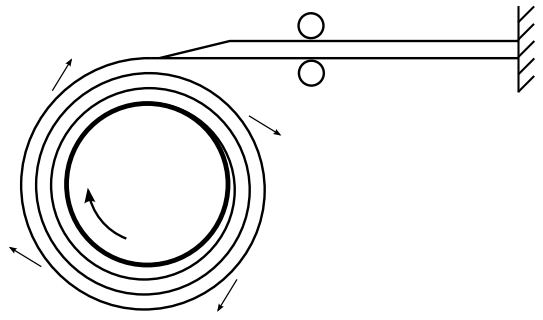

initial state

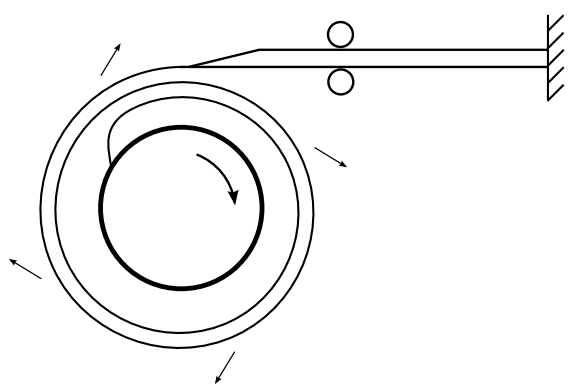

Figure 4.3: The strain energy reduction in the coil for a certain angle turned when the boom coil undergoes this motion gives rise to the central spindle torque. The length of the coil remains the same but the angle subtended by the coil decreases and the radius of the coil increases.

\subsection{Self-deployment torque}

A general definition of torque $(T)$ is the change in energy for a change in angle rotated $\left(\frac{d U}{d \theta}\right)$ as shown in Equation 4.1.

$$
U=T \theta \quad \rightarrow \quad T=\frac{d U}{d \theta}
$$

Rimrott used the strain energy stored in a coiled boom to calculate the deployment torque and deployment force so that the velocity and time for a coiled boom to deploy could be found for a number of different deployment configurations (Rimrott, 1967; Upadhyaya, 1968). A similar method can be used to find the self-deployment torque and the central spindle torque. The selfdeployment torque can be found using the following equations. The strain energy for a tape spring coiled in the opposite sense is:

$$
U_{o p}=\frac{D b r_{x} \theta}{2}\left[\frac{1}{r_{x}^{2}}+\frac{1}{r_{y}^{2}}+\frac{2 \nu}{r_{x} r_{y}}\right]
$$

Where $D$ is the flexural rigidity of a plate, $b$ is the width of the tape spring, $\theta$ is the angle subtended by the tape spring, $\nu$ is the Poisson's ratio, $r_{x}$ is the coil radius and $r_{y}$ is the coil natural radius. Cancelling $r_{x}$ gives:

$$
U_{o p}=\frac{D b \theta}{2}\left[\frac{1}{r_{x}}+\frac{r_{x}}{r_{y}^{2}}+\frac{2 \nu}{r_{y}}\right]
$$

Differentiating with respect to $\theta$ gives the self deployment torque for a tape spring in opposite sense bending: 


$$
T_{\text {sdop }}=\frac{d U_{o p}}{d \theta}=\frac{D b}{2}\left[\frac{1}{r_{x}}+\frac{r_{x}}{r_{y}^{2}}+\frac{2 \nu}{r_{y}}\right]
$$

Carrying out the same operations with Equation 3.10 would give the self deployment torque for a tape spring in equal sense bending:

$$
T_{\text {sdeq }}=\frac{d U_{e q}}{d \theta}=\frac{D b}{2}\left[\frac{1}{r_{x}}+\frac{r_{x}}{r_{y}^{2}}-\frac{2 \nu}{r_{y}}\right]
$$

Equations 4.4 and 4.5 give the self-deployment torque for a coil at a given radius. Figure 4.4 plots the self-deployment torque for a boom coiled in the opposite sense and the self-deployment torque for a boom coiled in the even sense for a range of coil radii. It shows the boom coiled in the opposite sense has a higher self-deployment torque due to the extra strain energy from the Possion's ratio effect but the torques follow the same gradient over the range of coil radii. There is a torque minimum when the coil radius is the same as the natural radius of the tape spring. When the coil is below the natural radius the increase in torque comes from the greater strain energy of the tighter coil. When the coil is above the natural radius the increase in torque comes from the larger coil radius and hence the larger lever arm to the central spindle. Also for a certain angle turned there will be a greater length of tape spring changing from the coiled to the deployed state when the coil radius is larger than the natural radius.

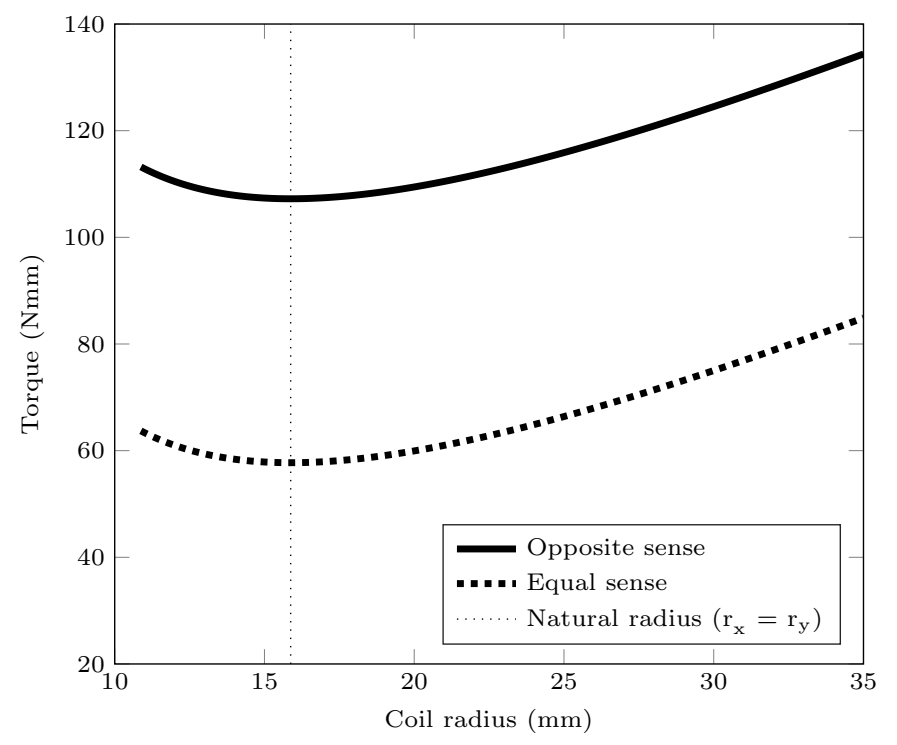

Figure 4.4: Self-deployment torque at different radii. The vertical line represents the tape spring's natural radius. Tape spring parameters: width $=31.8 \mathrm{~mm}$, thickness $=0.13 \mathrm{~mm}$, Young's modulus $=205 \mathrm{GPa}$, Poisson's ratio $=0.3$, and natural radius $\left(r_{y}\right)=15.9 \mathrm{~mm}$.

\subsection{Central spindle torque}

The central spindle torque can be found using a similar method used to find the self deployment torque. By using Equation 4.2 but changing $r_{x} \theta$ for $L$ (the length of the coil) the following equation gives the strain energy in a coil with opposite sense bending. 


$$
U_{o p}=\frac{D b L}{2}\left[\frac{1}{r_{x}^{2}}+\frac{1}{r_{y}^{2}}+\frac{2 \nu}{r_{x} r_{y}}\right]
$$

Equation 4.7 is for a boom that is coiled in the equal sense.

$$
U_{e q}=\frac{D b L}{2}\left[\frac{1}{r_{x}^{2}}+\frac{1}{r_{y}^{2}}-\frac{2 \nu}{r_{x} r_{y}}\right]
$$

Equations 4.6 or 4.7 can be used to find the strain energy stored in a coil undergoing the motion shown in Figure 4.3. In this scenario the length of the coil is fixed but the angle subtended by the coil and the coil radius can change. MATLAB can be used to plot the strain energy against the angle rotated. The gradient of this plot $\left(\frac{d U}{d \theta}\right)$ gives the central spindle torque. Figure $4.5(\mathrm{a})$ shows the strain energies for a coiled tape spring with equal and opposite sense bending for a coil initially with 15 turns, following the motion shown in Figure 4.3. As the boom blossoms the angle subtended decreases, the radius increases, and the strain energy decreases. Figure 4.5(b) is plotted using the gradient of the energy with respect to the angle subtended by the coil $\left(\frac{d U}{d \theta}\right)$ to give the central spindle torque as the coil blossoms. As blossoming occurs and the radius increases, the central spindle torque decreases. As with the self deployment torque the opposite sense coiled boom has a higher energy and a higher central spindle torque due to the Possion's ratio effect.

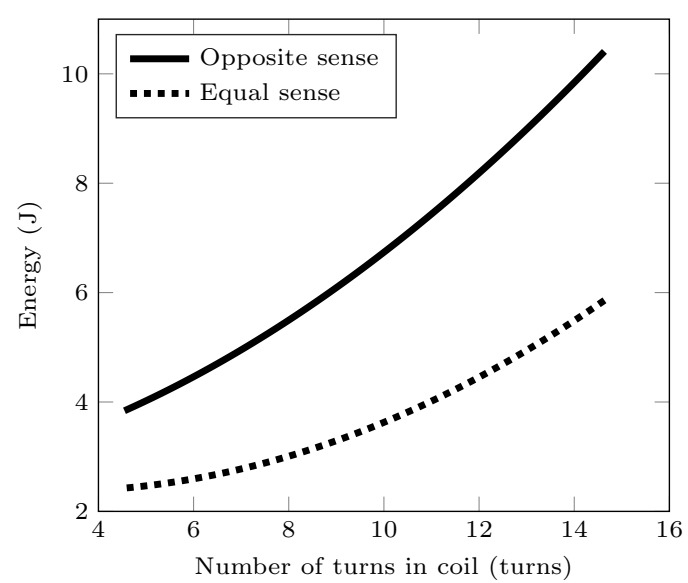

(a) Energy stored in coil during blossoming

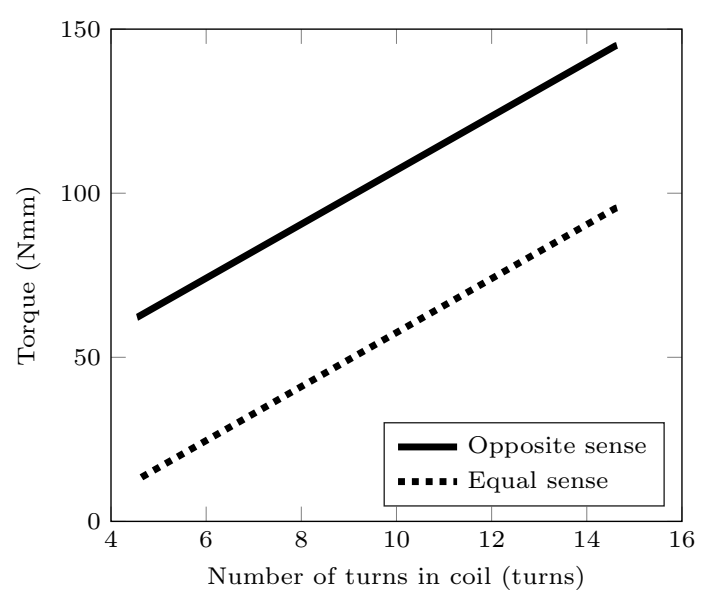

(b) Central spindle torque during blossoming

Figure 4.5: Tape spring parameters: length $=1 \mathrm{~m}$, width $=31.8 \mathrm{~mm}$, thickness $=0.13 \mathrm{~mm}$, Young's modulus $=205 \mathrm{GPa}$, Poisson's ratio $=0.3$, and natural radius $\left(r_{y}\right)=15.9 \mathrm{~mm}$.

Figure 4.6 plots the strain energy and the central spindle torque at different coil radii. Figure 4.6(a) shows how the strain energy stored in the coil decreases as the coil blossoms and the radius of the coil increases for both equal and opposite sense bending. Figure 4.6(b) shows the central spindle torque for coils with equal and opposite sense bending as the coil blossoms and the radius increases. The central spindle torque decreases as the radius increases. 


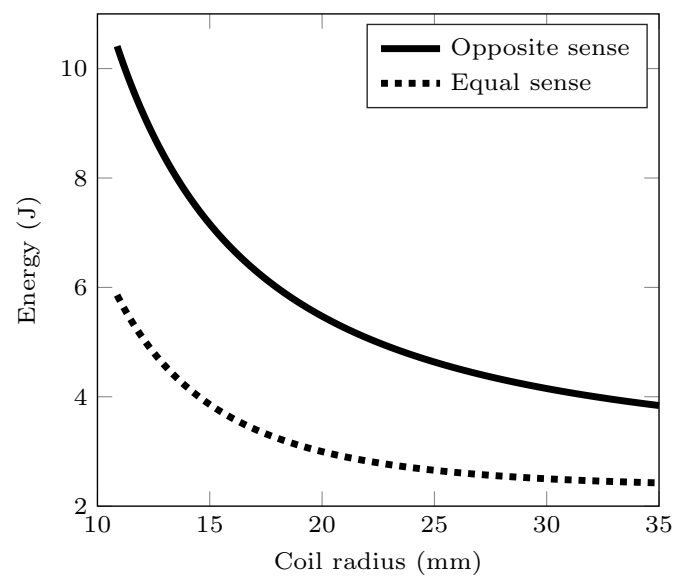

(a) Energy stored in coil during blossoming at different coil radii

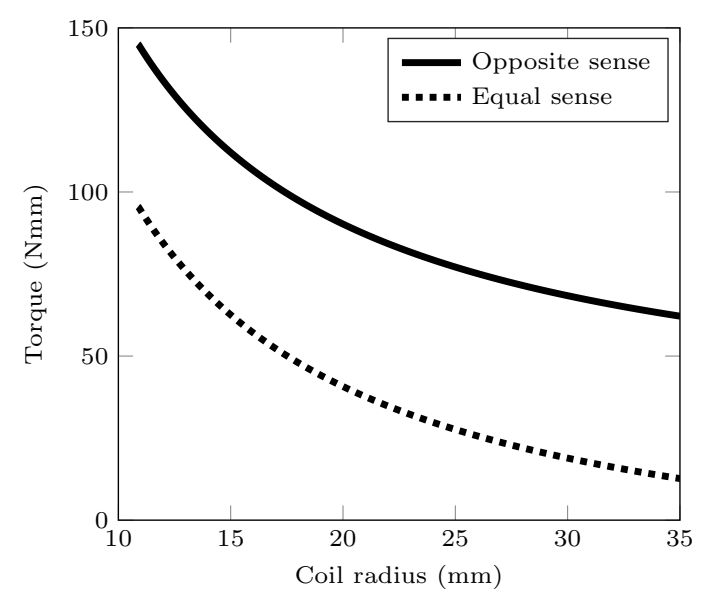

(b) Central spindle torque during blossoming at different coil radii

Figure 4.6: Tape spring parameters: length $=1 \mathrm{~m}$, width $=31.8 \mathrm{~mm}$, thickness $=0.13 \mathrm{~mm}$, Young's modulus $=205 \mathrm{GPa}$, Poisson's ratio $=0.3$, and natural radius $\left(r_{y}\right)=15.9 \mathrm{~mm}$.

\subsection{Central spindle torque and self-deployment torque com- parison}

Figure 4.7(a) shows the self-deployment torque and the central spindle torque during blossoming for a tape spring with opposite sense bending. Figure 4.7(b) shows the self-deployment torque and central spindle torque during blossoming for the same tape spring with equal sense bending. When a tape spring is coiled with no tip force and the central spindle is fixed, the coil will find its strain energy minimum when the coil radius is at the natural radius. At this strain energy minimum the central spindle torque and self deployment torque will be equal and opposite as is shown in Figures 4.7(a) and 4.7(b).

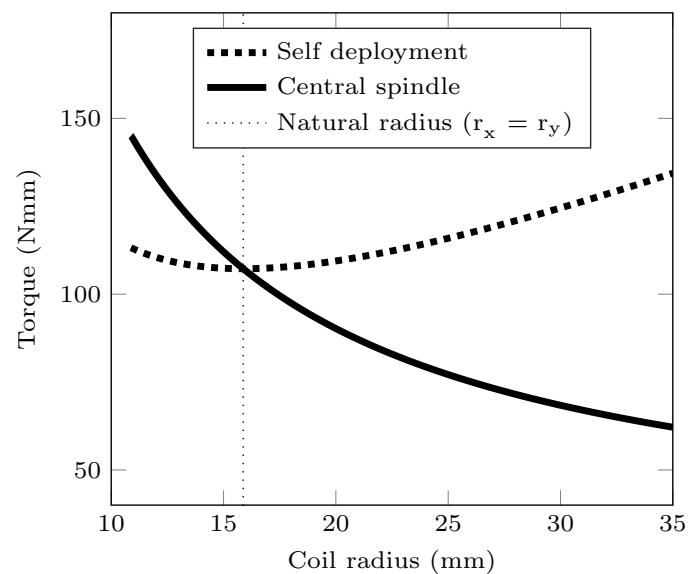

(a) Central spindle and self-deployment torques for opposite sense bending.

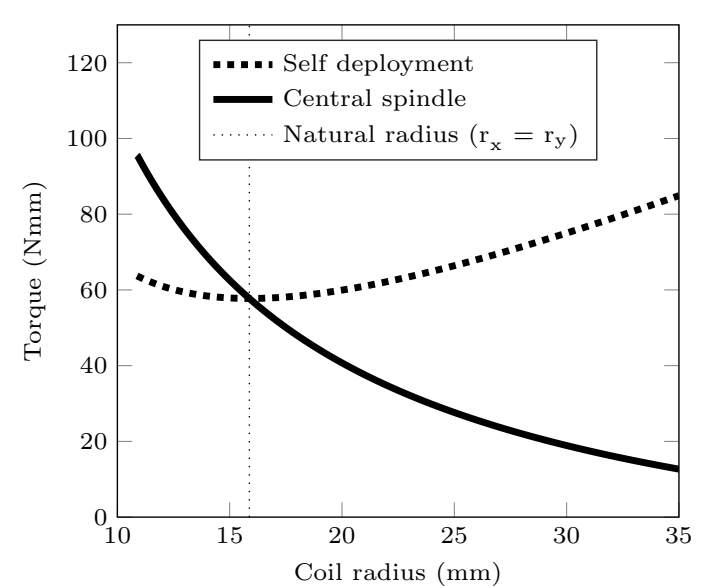

(b) Central spindle and self-deployment torques for equal sense bending.

Figure 4.7: Tape spring parameters: width $=31.8 \mathrm{~mm}$, thickness $=0.13 \mathrm{~mm}$, Young's modulus $=205 \mathrm{GPa}$, Poisson's ratio $=0.3$, and natural radius $\left(r_{y}\right)=15.9 \mathrm{~mm}$.

To cause the to coil increase or decrease in diameter a force must be applied to the tip of the boom. When a force is applied towards the coiled boom the radius will increase and when a force is applied away from the coiled boom the coil radius will decrease. The force applied to the tip to create a certain tip displacement or change in radius of the tape spring has been found in Chapter 3. As the radius increases, the self deployment torque increases and the central spindle torque decreases 
as is shown in Figures 4.7(a) and 4.7(b). Another way to find the tip force is to sum the forces and moments in the tape spring. When a tip force is applied towards the coil it acts in opposition to the self deployment torque at a distance of the radius of the coil. This gives rise to Equation 4.8, where $r_{o}$ is the outer radius of the coil, $F_{t i p}$ is the tip force, $T_{c s}$ is the central spindle torque and $T_{s d}$ is the self deployment torque.

$$
T_{c s}=T_{s d}-\left(F_{t i p} \times r_{o}\right)
$$

This can be rearranged to give:

$$
F_{t i p}=\frac{T_{s d}-T_{c s}}{r_{o}}
$$

Equation 4.9 gives the same tip force as Equation 3.13 in Chapter 3.

\subsection{Zero central spindle torque}

The central spindle torque can be found using another method, using Equations 4.10 and 4.11 (Calladine, 1989; Seffen and Pellegrino, 1999). $M_{o p}$ is the central spindle torque for a coil in opposite sense bending and $M_{e q}$ is the central spindle torque for a coil in equal sense bending. $D$ is the flexural rigidity of a plate, $b$ is the tape spring width, $\kappa_{x}$ is the longitudinal curvature, $\kappa_{y}$ is the tape spring natural curvature and $\nu$ is the Poission's ratio. This method gives the same result as finding the gradient of the strain energy - angle plot using Equations 4.6 and 4.7, but cannot be extended to incorporate the coil spiral geometry. However the method is useful for exploring the central spindle torque as the coil radius increases significantly in size. Figure 4.8(a) shows the strain energy for a coil with equal and opposite sense bending as the coil radius increases significantly. The equal sense bending shows a strain energy minimum between a coil radius of $40 \mathrm{~mm}$ and $60 \mathrm{~mm}$. Figure 4.8(b) shows the central spindle torque of the same coil in equal and opposite sense bending, indicating that the central spindle torque actually becomes negative at a radius of approximately $55 \mathrm{~mm}$. The energy minimum shown in Figure 4.8(a) corresponds to the point where the central spindle torque changes from positive to negative in Figure 4.8(b).

$$
\begin{aligned}
& M_{o p}=D b\left(\kappa_{x}+\nu \kappa_{y}\right) \\
& M_{e q}=D b\left(\kappa_{x}-\nu \kappa_{y}\right)
\end{aligned}
$$




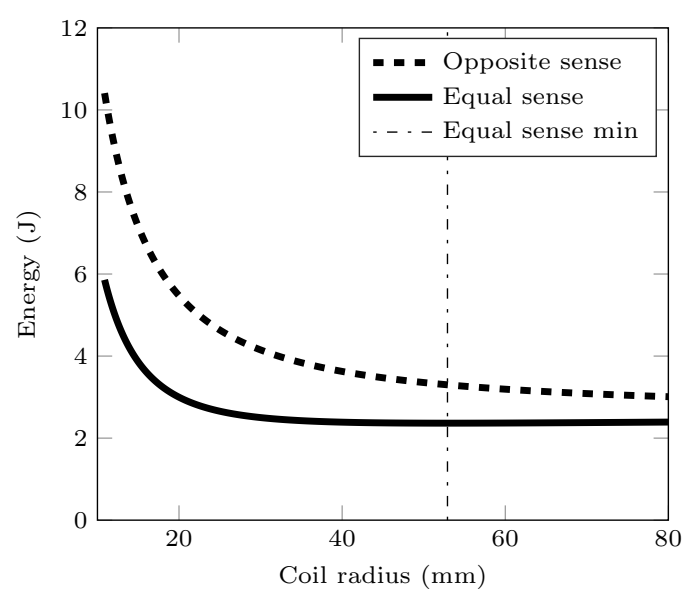

(a) The strain energy stored in a coil held at different radii for both equal and opposite sense bending.

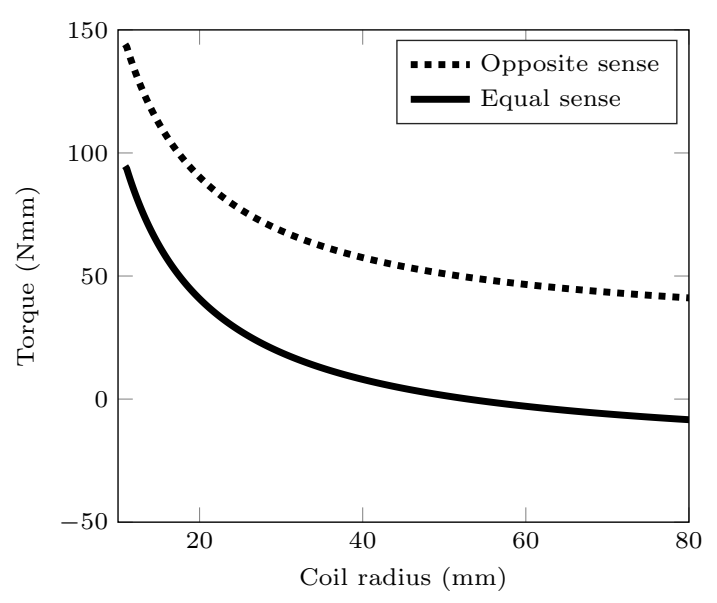

(b) The central spindle torque for a coil held at different radii for both equal and opposite sense bending.

Figure 4.8: The strain energy and central spindle torque for equal and opposite sense bending for a tape spring during blossoming following the motion show in Figure 4.3. Tape spring parameters: width $=31.8 \mathrm{~mm}$, thickness $=0.13 \mathrm{~mm}$, Young's modulus $=205 \mathrm{GPa}$, Poisson's ratio $=0.3$, and natural radius $\left(r_{y}\right)=15.9 \mathrm{~mm}$, length $=1 \mathrm{~m}$.

The result of the central spindle torque going to zero and then becoming negative seems counterintuitive as it would seem logical that a boom would carry on uncoiling until it became straight. Figure 4.8 suggests that if the coil was fixed at the tip and the central spindle was allowed to uncoil, it would stop uncoiling at a radius between $40 \mathrm{~mm}$ and $60 \mathrm{~mm}$. The reason for the torque going to zero and then becoming negative is the Poission's ratio effect in the boom. Figure 4.9 shows the changes in curvature that an equal sense coiled boom goes through to form a coil. Figure 4.9(a) shows the boom in the unstressed state with no longitudinal curvature but with the natural radius, $r_{y}$. To be coiled, the lateral curvature has to be flattened. When this happens there is a tensile stress on the inside face (previously concave) of the boom and compressive stress on the outside face of the boom, as shown in Figure 4.9(b). The large arrows represent the tensile and compressive forces caused by the flattening of the boom. On the inside face, due to the Poission's ratio effect, the elongation causes a tensile stress along the length of the boom. On the outside face the compression causes a compressive stress along the length of the boom. The stresses caused by the Possion's ratio effect are shown by small arrows. The two stresses caused by the Poisson's ratio effect tend to cause the tape spring to form a curvature in the longitudinal direction, $r_{x}$, as shown in Figure 4.9(c). If the longitudinal curvature is at the correct value it will cancel out the stresses from the Poission's ratio effect. The value of the coil curvature that gives a zero central spindle torque can be found by setting Equation 4.11 to zero, as shown in Equation 4.12. This gives a coil radius value of $53 \mathrm{~mm}$ which correlates to zero torque shown in Figure 4.8.

$$
\kappa_{x}=\nu \kappa_{y} \quad \rightarrow \quad \frac{1}{r_{x}}=\nu \frac{1}{r_{y}} \quad \rightarrow \quad r_{x}=\frac{r_{y}}{\nu}
$$



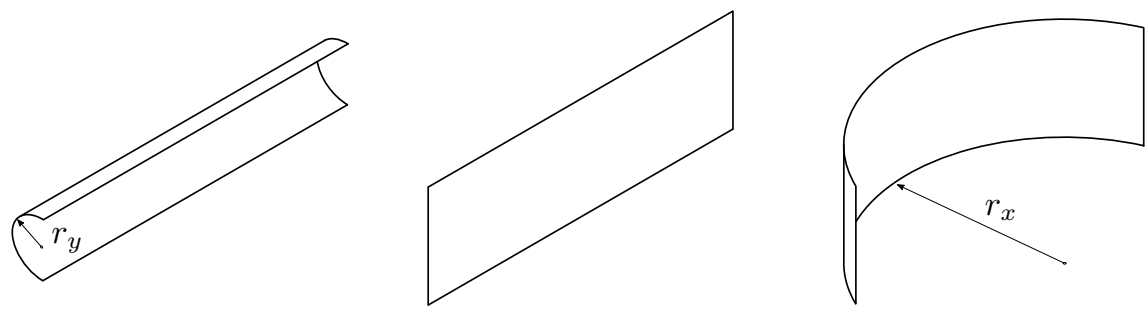

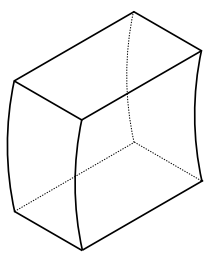

(a)

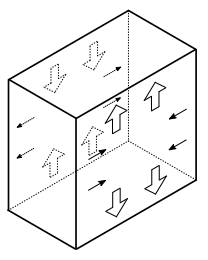

(b)

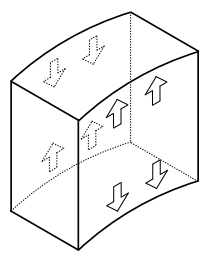

(c)

Figure 4.9: The changes in curvature that result in a zero central spindle torque. The top diagrams show the changes in curvature when a boom is coiled and the bottom diagrams show the stresses in a small segment of the boom at each stage. (a) The boom is in its unstressed state with no longitudinal curvature but with a lateral curvature, $r_{y}$. (b) The boom is completely flat. (c) The boom has formed a coil with a coil radius, $r_{x}$.

As the coil radius continues to expand the central spindle torque will asymptote to two different values for equal and opposite sense bending. These values can be found by setting the coil curvature $\left(\kappa_{x}\right)$ to zero in Equations 4.10 and 4.11. Figure 4.10 shows how the central spindle torque tends towards these values as the coil radius is increased.

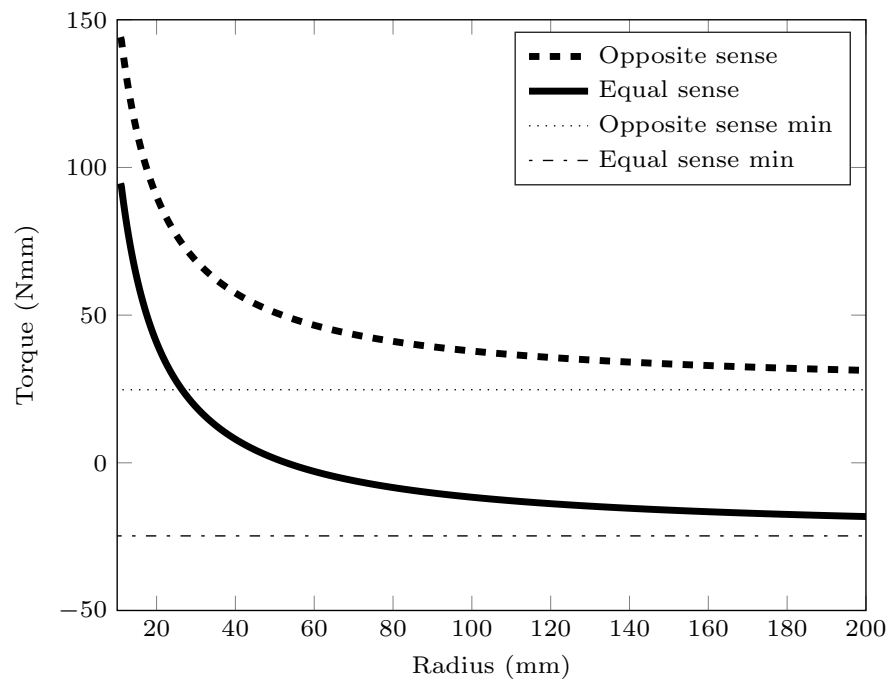

Figure 4.10: Self deployment torque as the coil radius is increased significantly for equal and opposites sense bending. Tape spring parameters: width $=31.8 \mathrm{~mm}$, thickness $=0.13 \mathrm{~mm}$, Young's modulus $=205 \mathrm{GPa}$, Poisson's ratio $=0.3$, and natural radius $\left(r_{y}\right)=15.9 \mathrm{~mm}$.

The strain energy minimum giving rise to a second stable state for a boom made of an isotropic material has also been noted in research on the bistability of composite booms (Guest and Pellegrino, 2006). In the paper an energy minimum for an isotropic tape spring was also found to occur with a coil radius of approximately $1 / 3$ of the natural radius. The paper noted that the energy minimum was at a saddle point which would result in a tape spring exhibiting an unstable torsional twisting mode which can be seen by trying to coil a tape measure at $1 / 3$ of its natural radius. This unstable torsional twisting mode can also be seen in the case of a coiled boom. When the outer radius of a tape spring coil increases significantly the coil starts to form a "50 pence" 
effect as noted in another study on bistability (Fernandez et al., 2014). It appears from these two studies that a torsional twisting mode will take effect before the coil will get to a size where the zero central spindle torque will be seen in reality.

\subsection{Spiral geometry}

Until now the central spindle torque calculations have been performed based on the assumption that the radius of curvature of the coiled boom is constant from root to tip for simplicity. For a more accurate prediction of the central spindle torque, the strain energy stored in a coiled boom taking into account the spiral geometry and the strain energy stored in the compression springs can be used. This gives a more accurate central spindle torque as it gives a more accurate strain energy for coiled booms with longer lengths and hence a greater change in radius from the inside to the outside of the coil. The same methods used to find the strain energy stored in the coil with Archimedean spiral geometry and compression springs to give the tip force during blossoming have been used. The area under a curvature - length plot was found and used along with Equation 4.3 to give the strain energy stored in the coil. MATLAB was also be used to find the displacement and hence strain energy stored in the compression springs. The gradient of the compression spring and coil strain energy with respect to angle turned would then give the central spindle torque.

The self-deployment torque also needs some adjustment to take into account the change in coil radii from the inside of the coil to the outside. As the self-deployment torque originates from the tape spring changing from its stored state to its deployed state, the radius that should be used for $r_{x}$ in Equations 4.4 and 4.5 should be the outer coil radius. These two changes allow a more accurate prediction of the central spindle torque and the self-deployment torque. More detail on the method used to find the central spindle torque can be found in Appendix B.

\subsection{Experiments}

During the practical tests to find the tip force shown in Chapter 3 the central spindle torque was also recorded. The results are shown in Figures 4.11 to 4.16. The figures compare the experimentally measured central spindle torque to the central spindle torque predicted by the theory detailed in Section 4.2 and Appendix B. The boom tip was held in place by the force sensor and the central spindle was turned by an electric motor. The central spindle was turned in increments of $36^{\circ}$, with a torque measurement taken each time. For each set of parameters the test was run three times. This is shown in the following figures as experimental results 1, experimental results 2 and experimental results 3. A negative central spindle torque is taken as the central spindle stopping the boom from deploying. This would be the tape spring trying to turn the central spindle in a clockwise direction in Figure 4.1. In some of the figures the practical results have a positive torque, this is where the central spindle is driving the turning of the coiled boom and is shown as a clockwise torque applied to the coiled boom in Figure 4.1.

Figure 4.11 shows the results for boom type A with 5 coils. The practical results show less clockwise (negative) torque than the theory predicts in all the cases. However, the practical results follow the decreasing negative torque gradient that the theory predicts. There is a trend of the clockwise torque decreasing further as the compression spring force is increased. There is also a periodic increase and decrease in the torque as opposed to a constant decrease in torque as the theory suggests. This is associated with the single boom not being concentric with the central spindle. This was also seen with the tip forces in the single boom case as is shown in Figure 3.32. Due 
to the asymmetry of the forces within the coil when there is only a single boom the boom coil tends to become non concentric with the central spindle. This causes the tip force to periodically increase and decrease, and it seems this also has an effect on the central spindle torque.

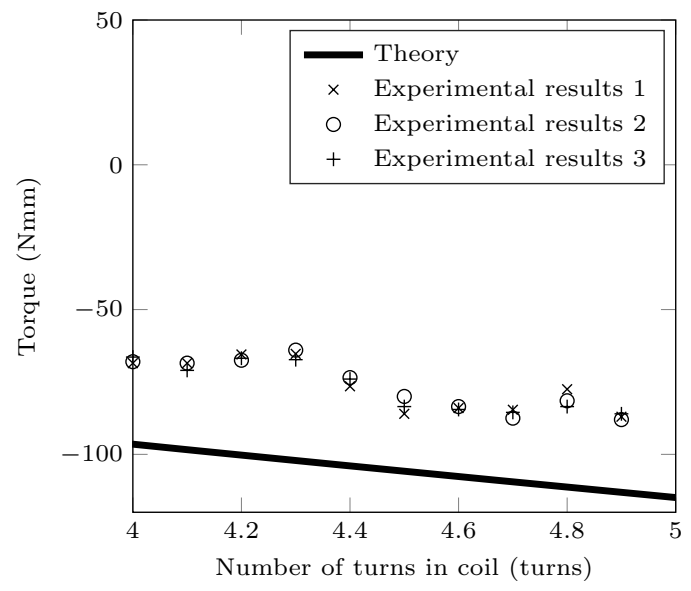

(a) $1 \mathrm{~N}$ compression spring force.

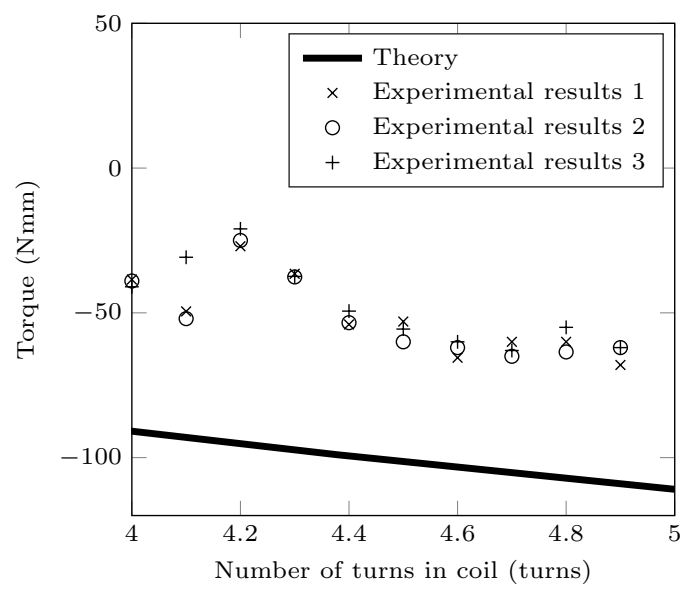

(c) $3 \mathrm{~N}$ compression spring force.

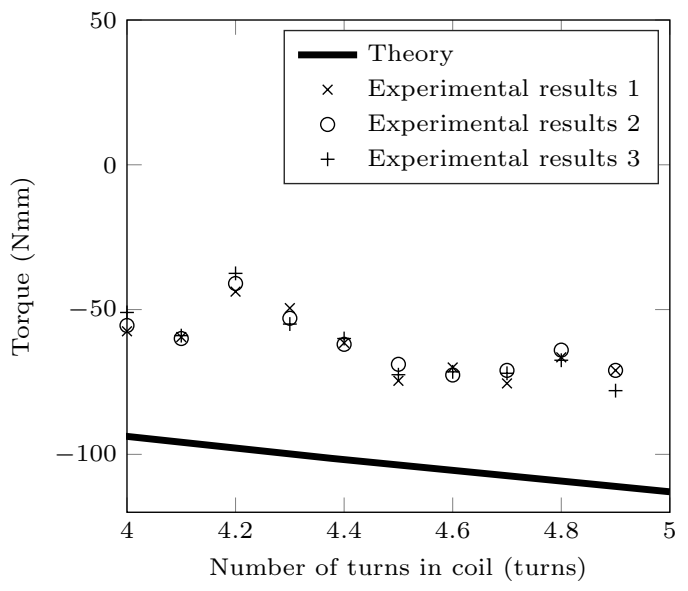

(b) $2 \mathrm{~N}$ compression spring force.

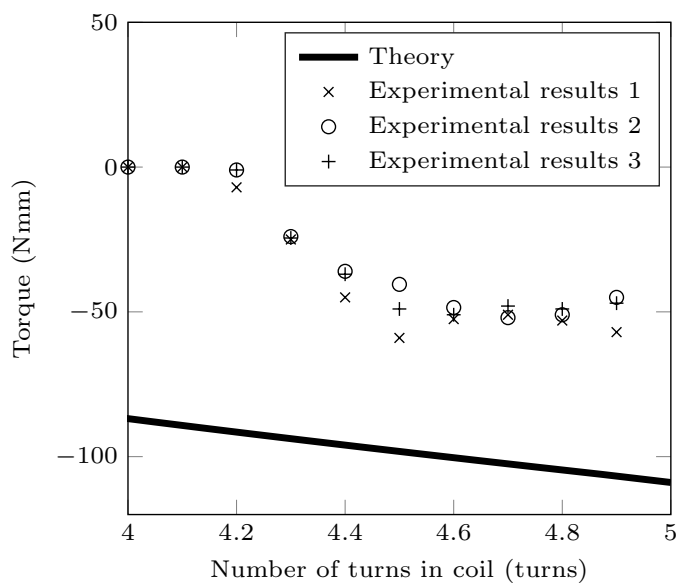

(d) $4 \mathrm{~N}$ compression spring force.

Figure 4.11: Results for boom type A, using a single boom and 5 coils.

The results for boom type A using two booms are shown in Figure 4.12. The theoretical central spindle torque has almost doubled compared to the case with the single boom as the strain energy from the coiled booms has doubled. However the strain energy from the compression rollers remains almost the same as for the case with a single boom as, apart from the outside coil radius being slightly larger because of the doubling of the thickness of the coil due to there being two booms instead of one, the displacement of the compression rollers remains similar. The practical results show a similar pattern with an almost doubling in torque compared to the results from the single boom. The practical results seem to show a more linear decrease in clockwise torque as blossoming occurs compared to the results with the single boom which tended to oscillate due to the coil not being concentric with the central spindle. The same trend of a decrease in clockwise torque as the compression roller force increases is also seen. 


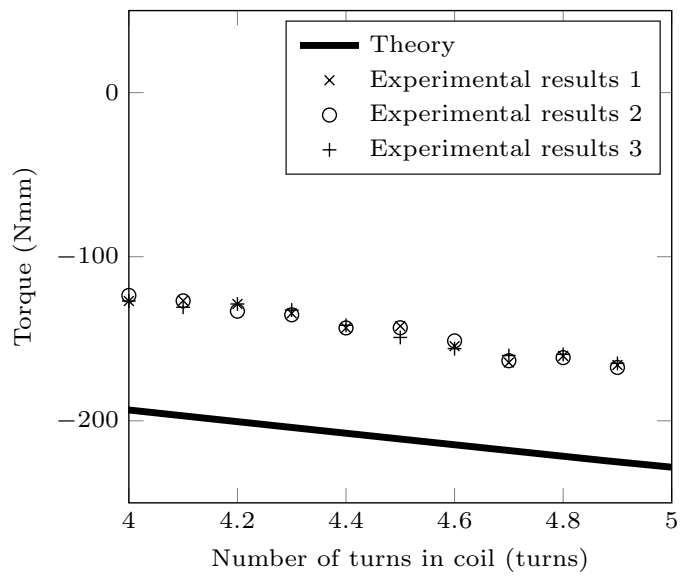

(a) $1 \mathrm{~N}$ compression spring force.

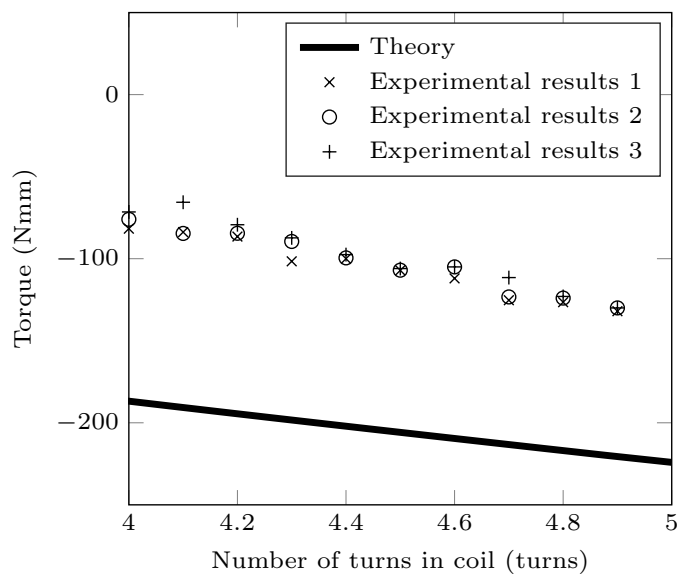

(c) $3 \mathrm{~N}$ compression spring force.

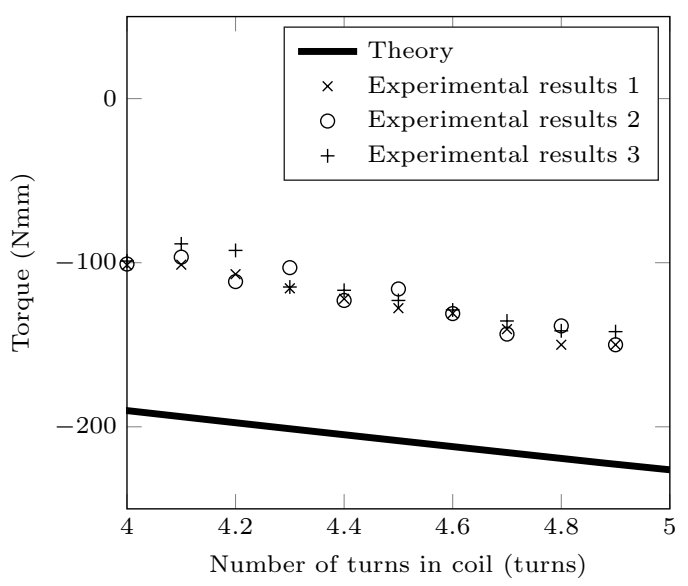

(b) $2 \mathrm{~N}$ compression spring force.

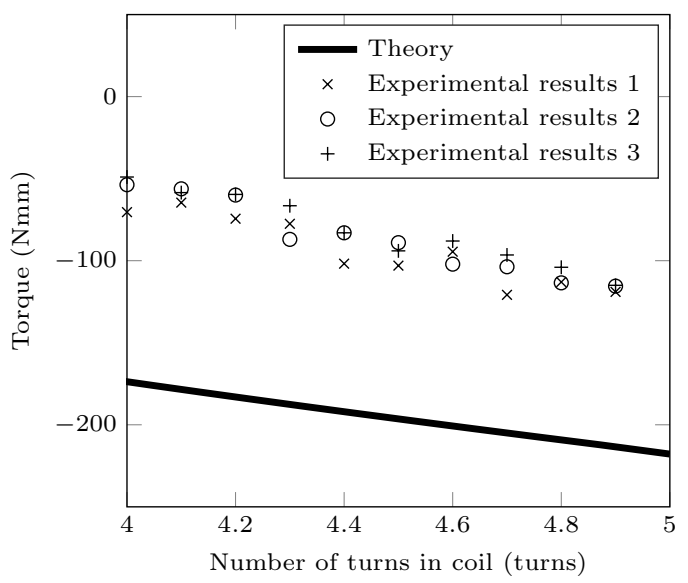

(d) $4 \mathrm{~N}$ compression spring force.

Figure 4.12: Results for boom type A, using two booms and 5 coils.

The results for boom type B show smaller central spindle torques for both practical and theoretical results, as shown in Figure 4.13. This is caused by boom type B having less strain energy due to the smaller width of the boom and the larger natural cross section radius compared to boom type A. There also seems to be a trend of the clockwise torque decreasing to become positive during blossoming between 4.8 to 4.4 turns in the coil. This is possibly due to the coils being more tightly wrapped causing more friction between the layers meaning it would take a more positive central spindle torque before blossoming would occur. After this number of turns $(4.4-4)$ the central spindle torque seems to reduce again. This may be caused by the loosening of the coils as blossoming occurred. Before and after this 'hump' where the negative torque decreases to become positive, the gradient of the practical torque seems to match the theoretical predictions. A similar trend of a decreasing clockwise torque is observed as the compression spring force is increased. 


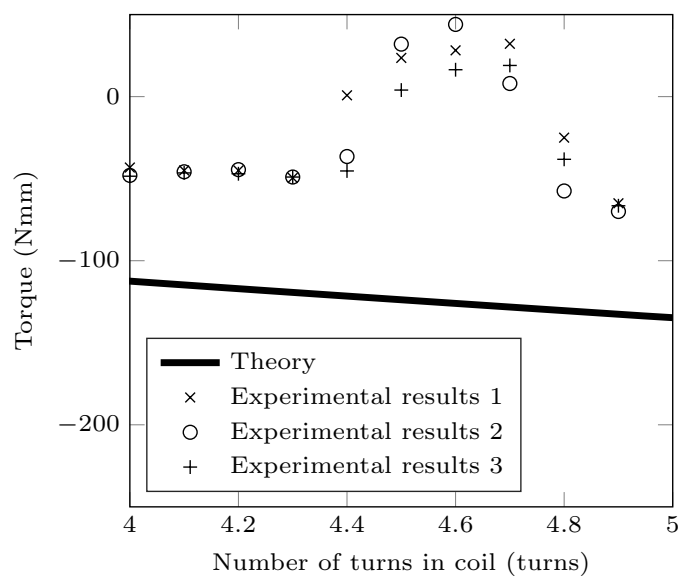

(a) $1 \mathrm{~N}$ compression spring force.

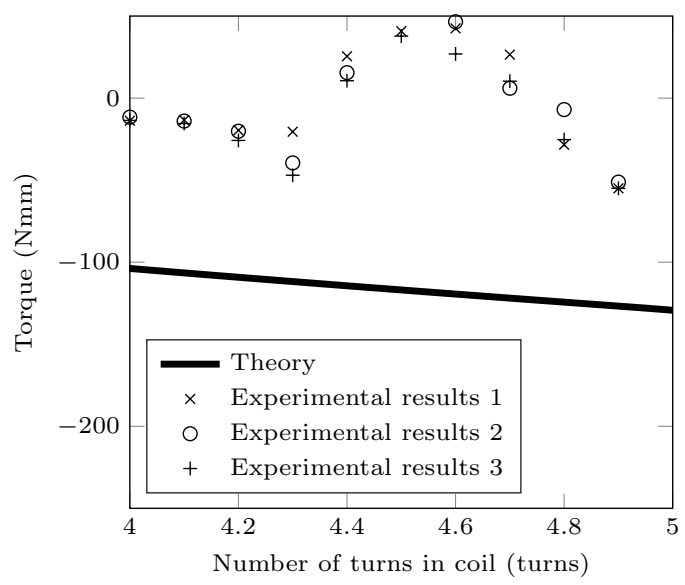

(c) $3 \mathrm{~N}$ compression spring force.

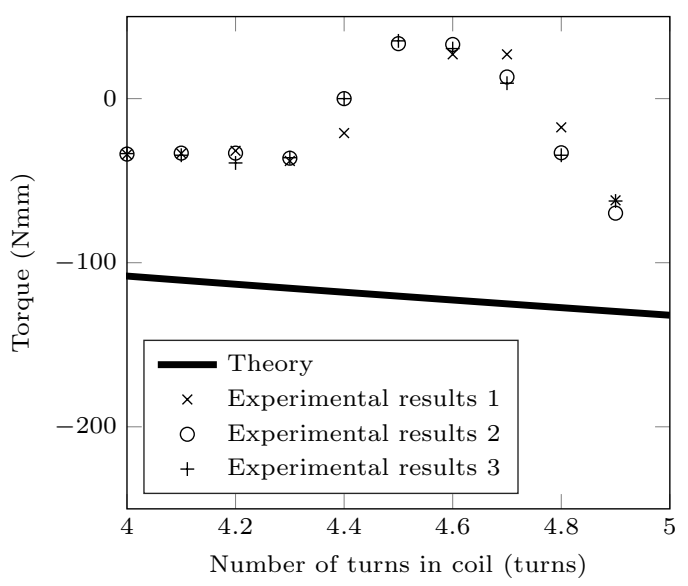

(b) $2 \mathrm{~N}$ compression spring force.

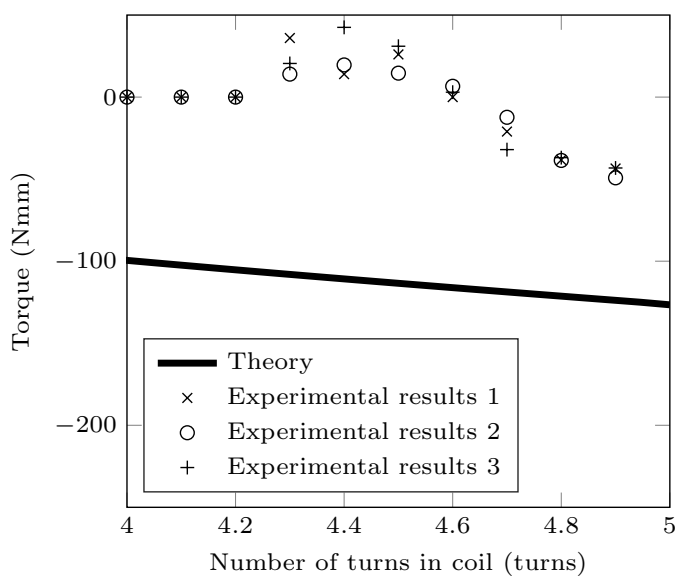

(d) $4 \mathrm{~N}$ compression spring force.

Figure 4.13: Results for boom type B, using two booms and 5 coils.

The practical results for the experiments with boom type B and 10 coils seem to have less pronounced 'humps' in the results compared to the experiments with 5 coils, as shown in Figure 4.14. The practical results seem to follow a similar trend to earlier cases in that the increase in compression roller force leads to a decrease in the clockwise force and in the case of $4 \mathrm{~N}$ of compression roller force the torque turns positive. 


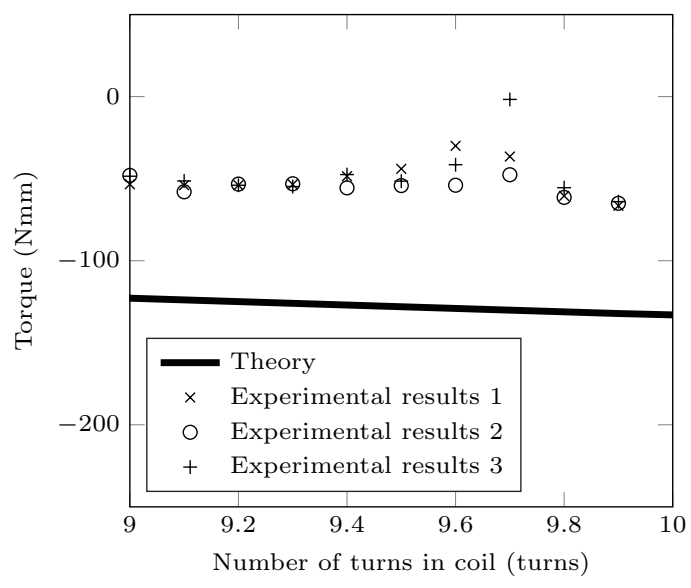

(a) $1 \mathrm{~N}$ compression spring force.

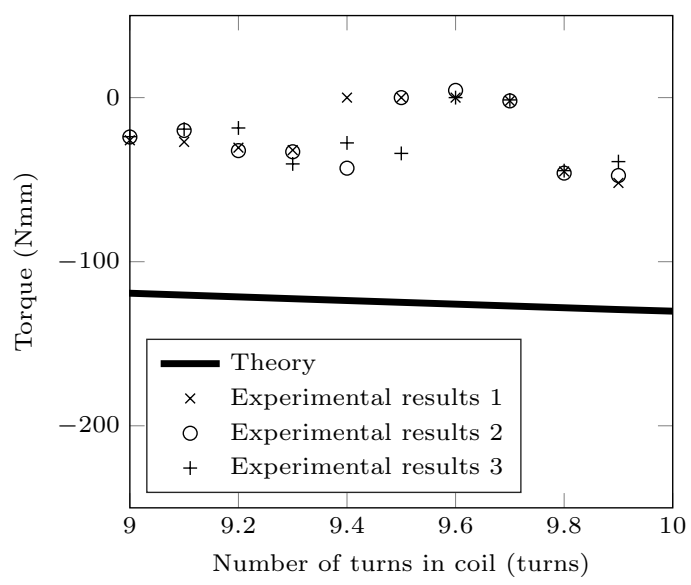

(c) $3 \mathrm{~N}$ compression spring force.

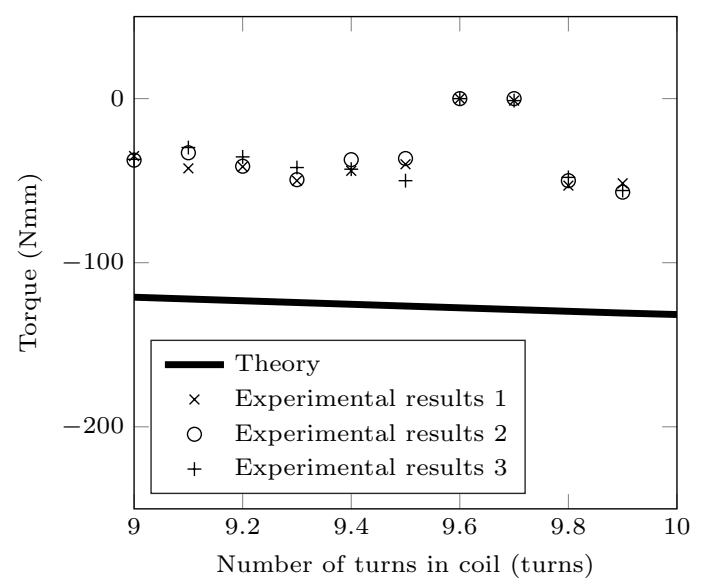

(b) $2 \mathrm{~N}$ compression spring force.

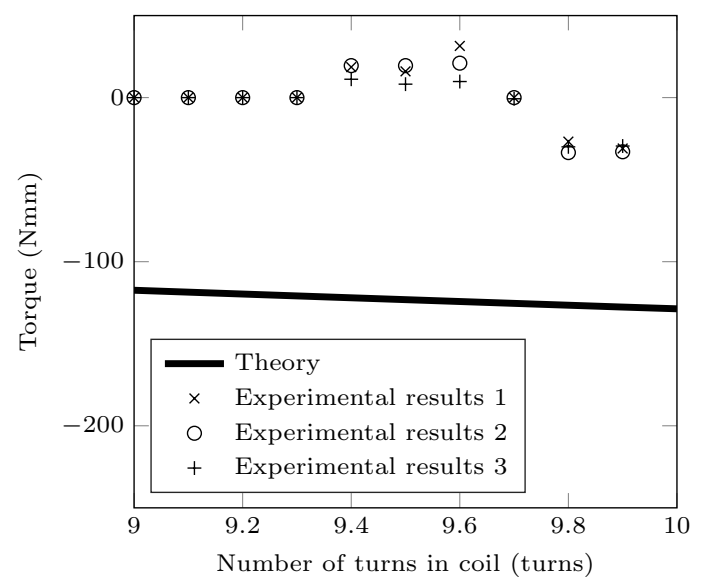

(d) $4 \mathrm{~N}$ compression spring force.

Figure 4.14: Results for boom type B, using two booms and 10 coils.

The results for 15 and 20 coils using boom type B follow a similar pattern to the results for 10 coils, as shown in Figure 4.15 and Figure 4.16. The 'humped' decrease in clockwise torque caused by the tightness of the boom coil from approximately half a turn of blossoming is less pronounced than the case with 5 coils but is still observable. The general trend of less clockwise force as the compression roller force increases is seen in all the cases and in some of the cases the central spindle torque turns positive. 


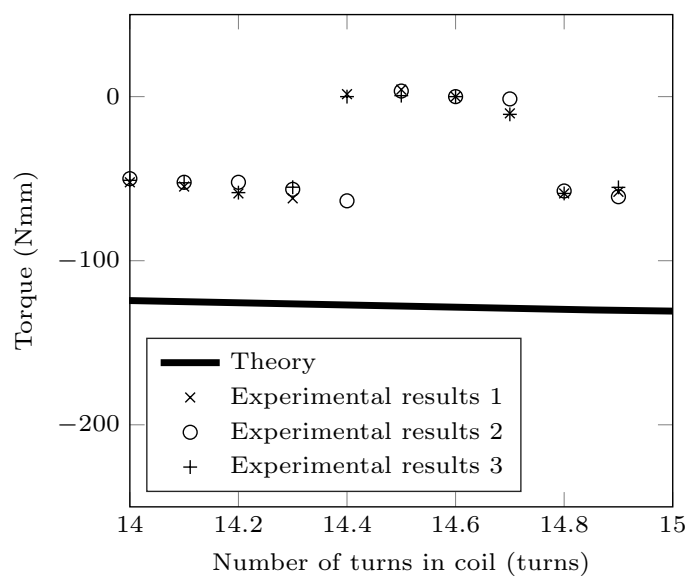

(a) $1 \mathrm{~N}$ compression spring force.

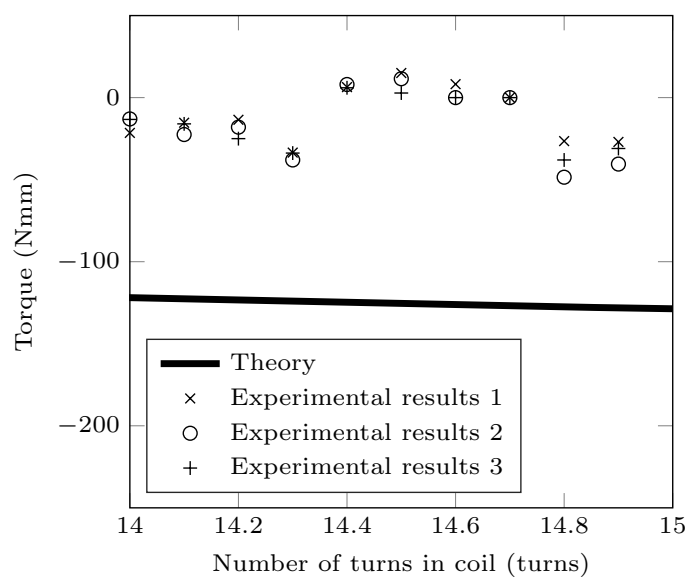

(c) $3 \mathrm{~N}$ compression spring force.

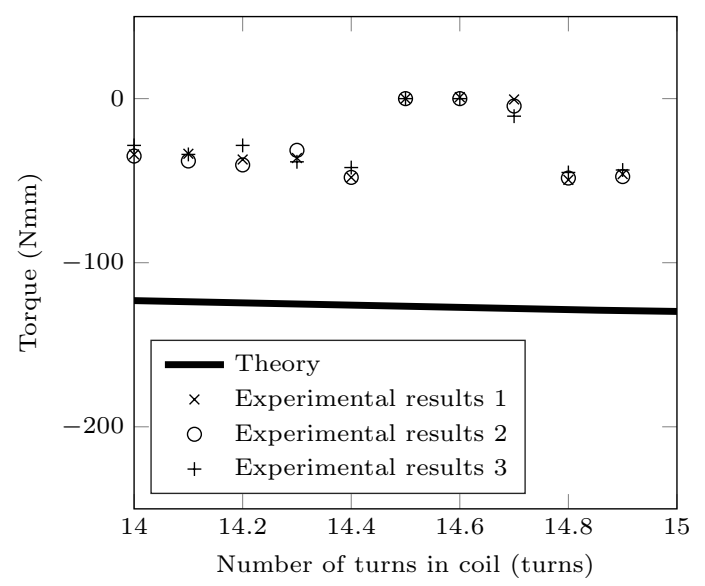

(b) $2 \mathrm{~N}$ compression spring force.

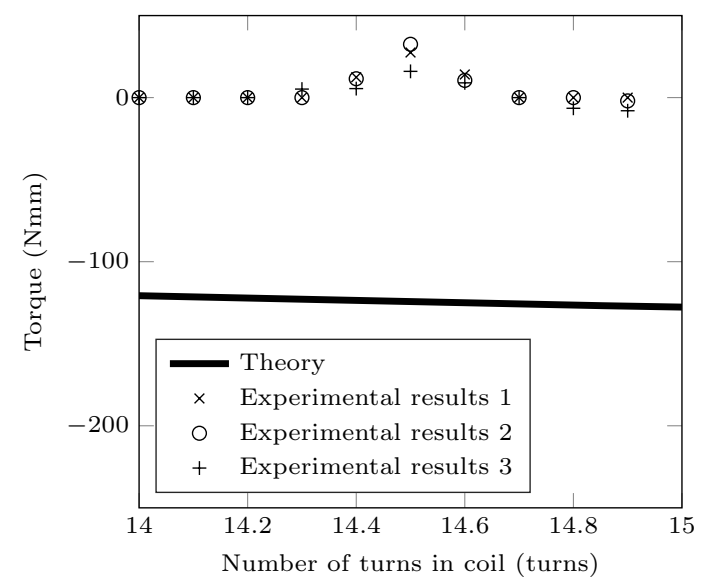

(d) $4 \mathrm{~N}$ compression spring force.

Figure 4.15: Results for boom type B, using two booms and 15 coils. 


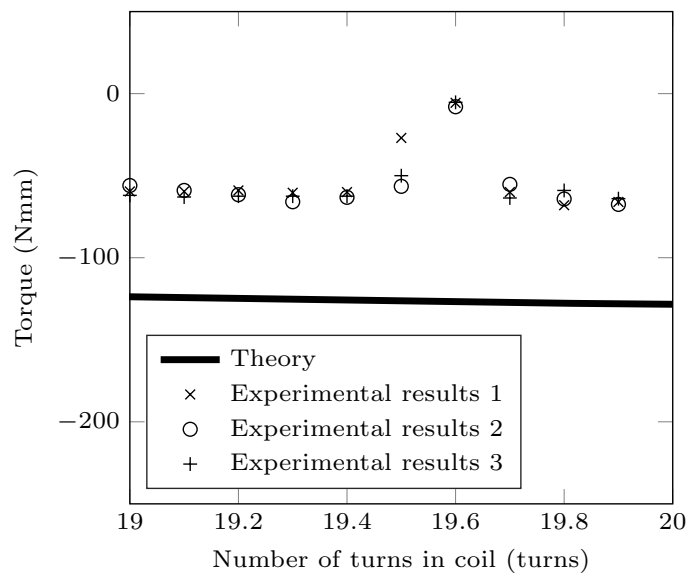

(a) $1 \mathrm{~N}$ compression spring force.

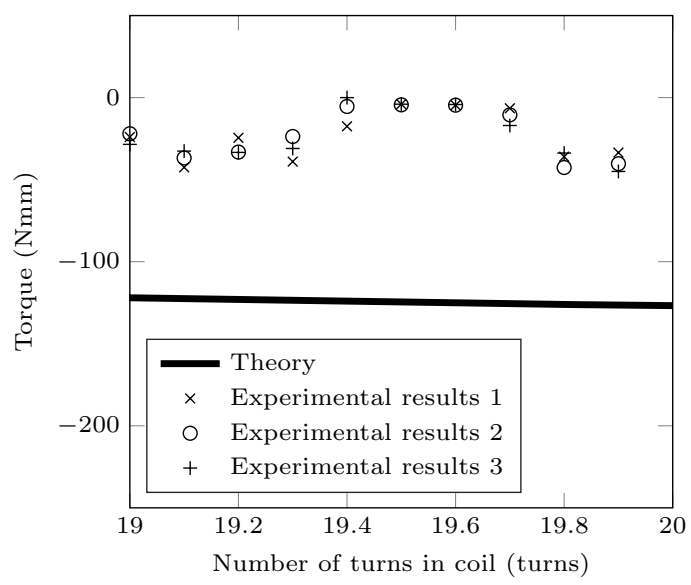

(c) $3 \mathrm{~N}$ compression spring force.

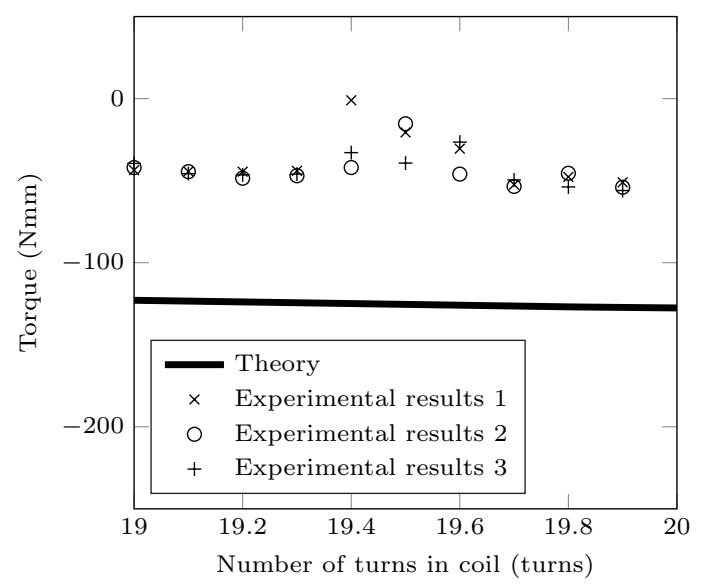

(b) $2 \mathrm{~N}$ compression spring force.

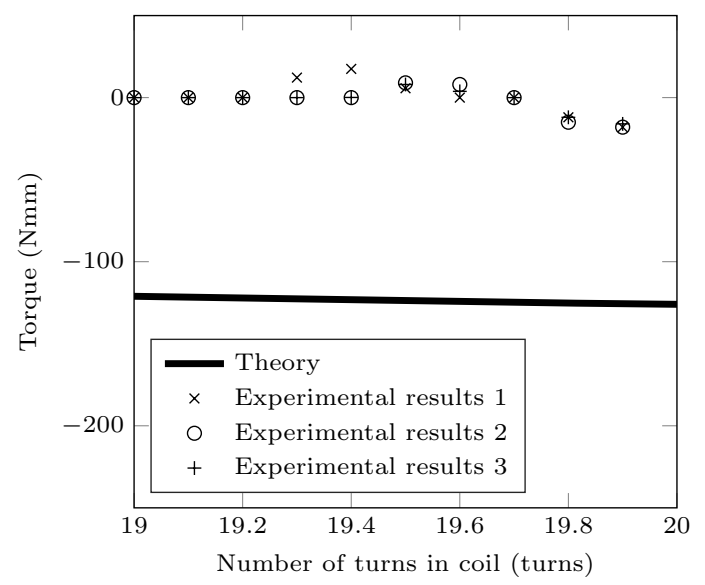

(d) $4 \mathrm{~N}$ compression spring force.

Figure 4.16: Results for boom type B, using two booms and 20 coils.

\subsection{Central spindle and self-deployment torque conclusion}

In all of the figures showing the theoretical and practical central spindle torques there are some common themes. The theoretical central spindle torque depends on whether boom type A or type B is used and if just one boom or two booms are used. Boom type A has a higher central spindle torque compared to boom type B due to it being wider and having a smaller natural radius than boom type B. The compression spring force also had a small effect on the theoretical central spindle torque. The greater the compression spring force, the lower the clockwise torque, as the compression springs oppose the expansion of the coil and hence make the energy gradient of the coiled boom and compression springs less.

Two booms will have slightly more than double the central spindle torque compared to one boom. While doubling of the number of booms doubles the strain energy in the coil, the strain energy stored in the compression springs only increases a small amount due to the increase in coil diameter from the thickness of having two booms instead of one. The compression springs act to reduce the central spindle torque as they expand as blossoming occurs. In changing from one boom to two booms this reduction in central spindle torque caused by the compression springs has less of an effect as the compression springs have double the amount of strain energy to resist. This is the reason for the central spindle torque increasing by slightly more than double when changing from one boom to two.

The number of turns in the coil has an effect on the gradient of the central spindle torque during blossoming. The smaller the number of turns in the boom coil the greater the increase in coil radius 
for each turn of the central spindle. The larger the coil radius the smaller the central spindle torque is as the strain energy gradient is less at larger coil radii. This is the reason the more coils there are in the central spindle the less the central spindle torque changes during blossoming over a single turn.

The practical results also show some common themes. The general trend is for the central spindle torque to follow the gradient predicted by the theory but with a reduced torque. The practical results showing less torque than predicted by the theory is thought to be because of the friction between the layers of the coil. A negative torque is the coiled boom trying to unwind by turning the central spindle. Because of the friction between the coil layers some of the torque is absorbed within the coil. When the compression spring force is increased the practical results show the general trend of the clockwise torque decreasing further, suggesting that the friction between the layers is the cause of the decrease in torque. As the compression roller force is increased the force between each layer of the coil is increased making it harder for the coiled layers to slide past one another.

In the results for boom type B there appears to be a series of 'humps' where the clockwise torque decreases from the fairly linear practical results seen in the cases using boom type A. This is thought to be due to the booms being wrapped quite tightly when coiled; this would increase the friction force between the coil layers and during the blossoming process they would take half a turn to loosen. While the coil was tight the clockwise central spindle torque would be smaller and in some cases turn positive, with the central spindle actually driving the blossoming. When a small amount of blossoming had taken place the coils would loosen and the clockwise torque would return. It is thought that this effect was not seen in boom type A as it was wider and had a smaller natural radius, giving it a higher central spindle torque that could have overcome the effects caused by the tightness of the coils during coiling.

The important observation from the measurement of the central spindle torque is that in most cases the tip force arises due to the strain energy in coil trying to minimise and not because of the torque applied by the central spindle. In most cases the central spindle is holding the coil back from deploying, it is only in a few cases that the central spindle is actually driving the deployment. It would be useful to investigate increasing the compression roller force further to find the force that the central spindle torque would turn positive and consistently drive the deployment. Unfortunately the increase in compression roller force increased the tip force to the point where boom type B would buckle at its attachment point to the force sensor. To carry out further investigations a boom with a greater subtended cross section angle would have to be used. 


\section{Chapter 5}

\section{Coil motion}

When blossoming occurs the layers of the coiled boom slide past one another. The relative motion between each layer depends on the number of coils as well as the coil geometry. This chapter looks at the motion between the different layers of coiled boom and investigates how the coefficient of friction would affect the force needed to cause a boom to blossom.

\subsection{Geometry}

When blossoming occurs the layers of the coil slide past one another. The relative motion between each layer of the coil is dependent on the length of the coil and the number of turns in the coil. In the case of a small number of turns in the coil and a thin boom, the relative angular motion of various points along the length of the boom can be described by Equation 5.1. $N_{c}$ is the initial number of coils, $Q$ is the coil number from the outside, $\psi$ is the angle rotated by the central spindle and $\phi$ is the angle moved by each point.

$$
\phi=\frac{\psi Q}{N_{c}}
$$

This relative motion of points along the length of the boom during blossoming is shown in Figure 5.1. As the inside of the coil is turned each coil layer slides past the next layer out. The start of the coil is attached to the central spindle so moves through the same angle. The inner part of the coil $(\mathrm{Q}=3$ in Figure 5.1) moves through a large angle. Towards the outside of the coil the boom moves through a smaller angle and the end of the coil is stationary.
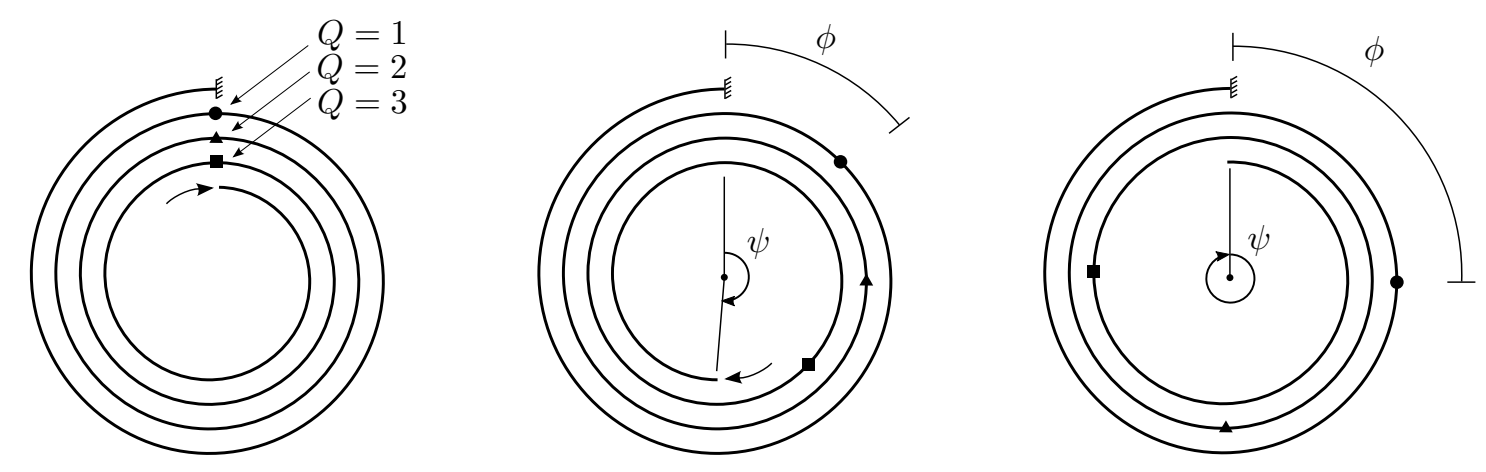

Figure 5.1: The relative motion of points on a coil with initially four turns. 
As Equation 5.1 shows, the further from the centre of the coil a point is, the smaller the angle it will rotate through for each rotation of the central spindle. This is shown in Figures 5.2 and 5.3. Figure 5.2 is for a coiled boom with four coils and represents the angles moved by the points depicted in Figure 5.1.

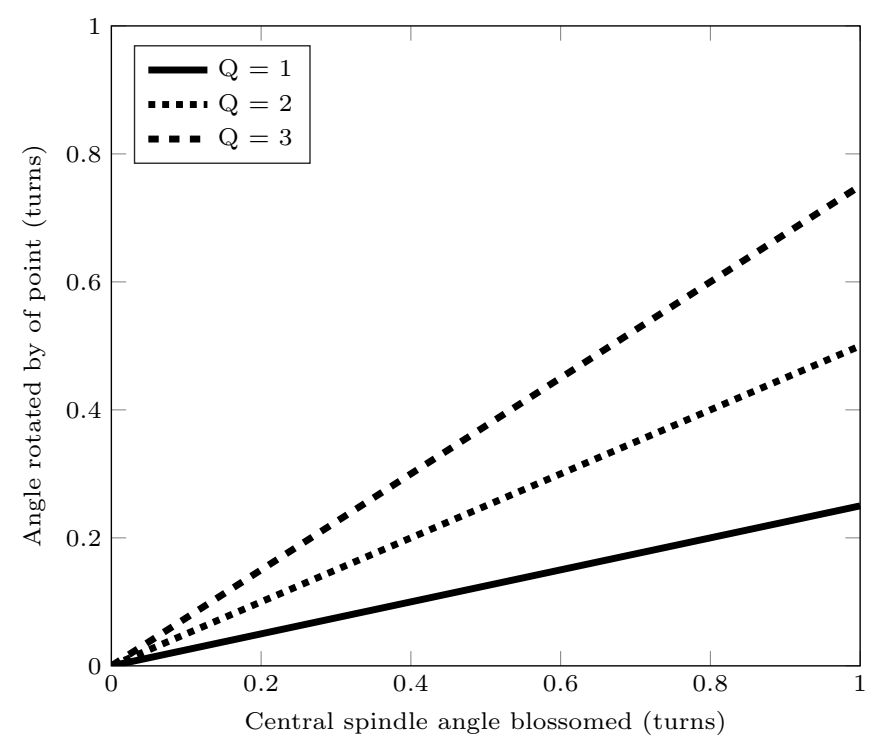

Figure 5.2: The rotation of three points within the coil shown in Figure 5.1.

Figure 5.3 shows the relative movement of points on a coiled boom with ten coils. In this case, compared to the previous case of a coiled boom with four coils, the outer most coil $(x=1)$ has moved through a smaller angle and the inner most coil $(x=9)$ has moved through a larger angle. The difference in rotation between each coil layer is smaller in the case of the coiled boom with ten coils than for the coiled boom with four coils. In Figure 5.2 the relative motion between each layer after one turn of the central spindle is one quarter of a turn. In the case of a coiled boom with 10 coils the relative position of each coil layer after one turn is one tenth of a turn. This shows that for a coil with a large number of turns the relative angle rotated and therefore the relative sliding motion between each coil layer will be smaller than for a coil with a small number of turns.

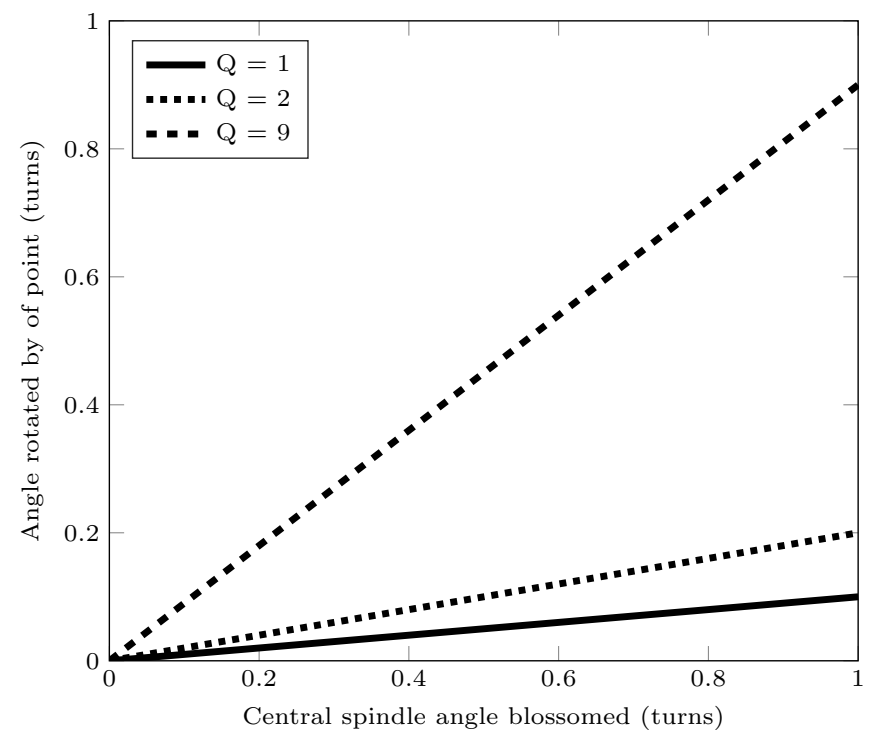

Figure 5.3: The rotation of three points on a coil with initially 10 turns.

As shown in the simple case above, the relative motion between each coil layer during blossoming 
increases as the number of coils decreases. In the extreme case where there is only one coil, the relative motion between the two layers is the same as the central spindle motion, this is shown in Figure 5.4. In the case of a coiled boom with more layers of coils the relative motion between each layer is less, as each subsequent layer is also moving, this is shown in Figure 5.5.
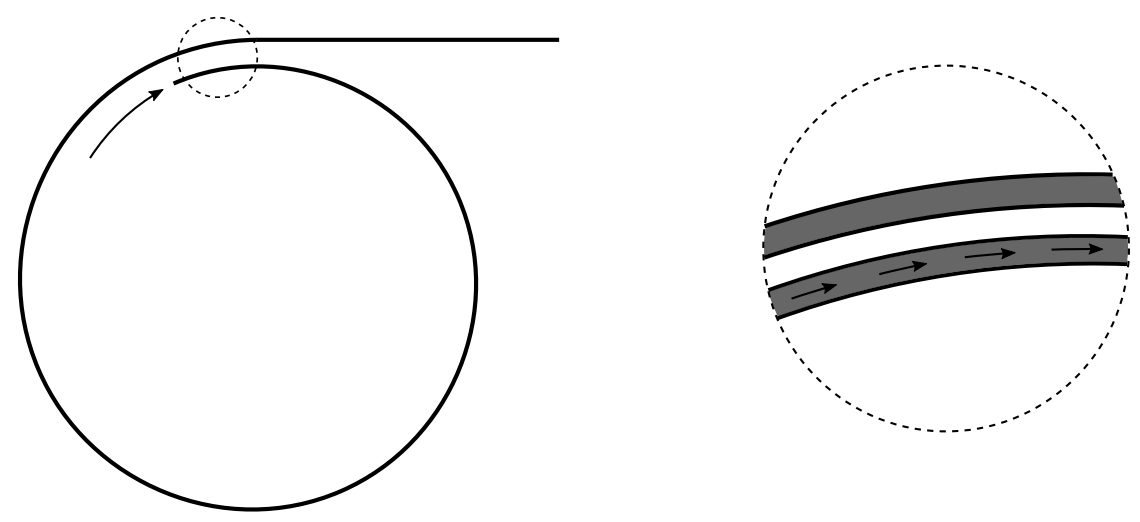

Figure 5.4: The relative motion between the inside and outside layer of the coil. The outside layer is stationary and the inner layer moves with the same motion as the central spindle.
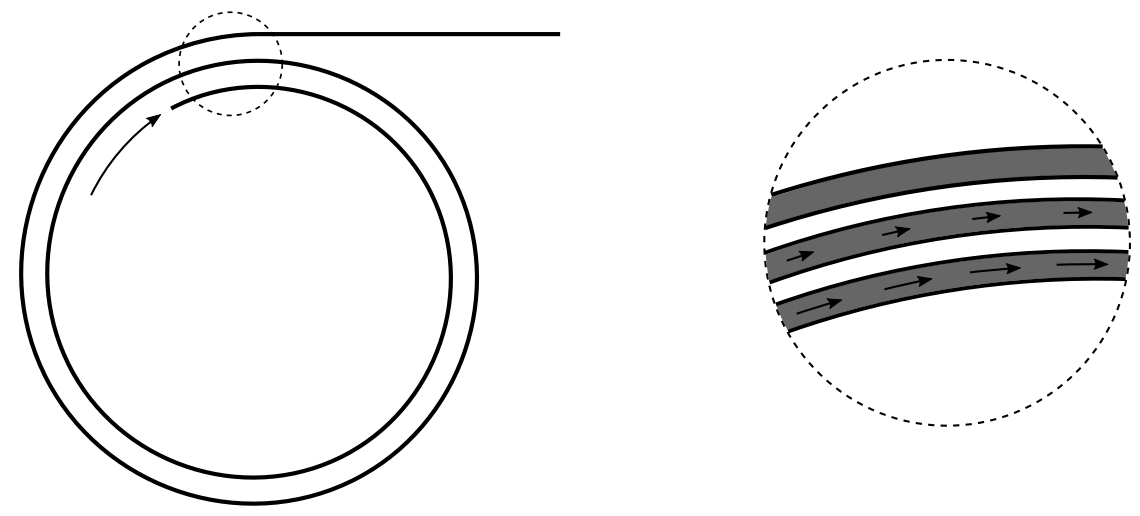

Figure 5.5: The relative motion between each layer of a coil with three turns. The outside layer is stationary, the middle coil moves with approximately half the motion of the central spindle and the inside layer moves with the same motion as the central spindle. This leads to an almost uniform sliding motion between each layer.

The relative distance slid between each layer for a certain angle blossomed can be estimated as the change in coil circumference per turn of the central spindle. By using the spiral geometry equations in Chapter 3, the change in circumference of the outside layer and the change in circumference of the inside layer during blossoming can be found. The sliding distance per turn of the inner and outer coils during the blossoming of a coiled with a boom with 22 turns is shown in Figure 5.6. Initially the angle subtended is 22 turns. As the central spindle is turned during blossoming the angle subtended decreases, as shown on the x-axis. The y-axis shows the instantaneous distance slid per rotation blossomed. This is the distance that one layer of the coil would slide relative to another coil layer if the coil radius stayed constant and the central spindle turned a complete turn. As can be seen, the inner and outer coils follow fairly similar curves, starting with a small distance slid per rotation blossomed and increasing as the angle blossomed increases. The inner coil always has a greater sliding distance per turn blossomed than the outside of the coil due to the inside radius increasing more quickly than the outside radius. This faster increase in the inner radius compared to the outside radius is because with each complete rotation blossomed the coiled boom has one less complete turn, and therefore the distance between the inside and outside radius must decrease by a single boom thickness, as is shown in Figure 5.7. 
The average coil distance slid for each turn of the central spindle can be described by Equation 5.2, this is also plotted in Figure 5.6. This equation finds an average distance slid by the coil layers per turn and is half way between the distance slid for the inside and outside of the coil. This motion has been termed relative coil sliding motion (RCSM). $L$ is the coil length and $N$ is the number of coils.

$$
R C S M=\frac{L}{N^{2}}
$$

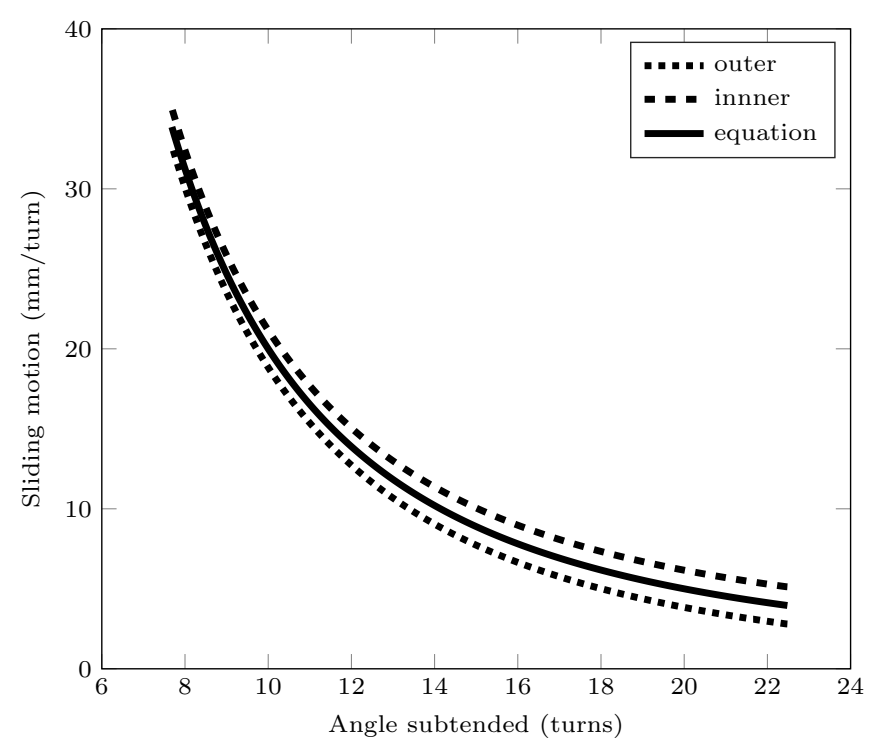

Figure 5.6: The change in sliding motion as the coil blossoms from a coil with 22 turns to a coil with 8 turns. The boom length is $2 \mathrm{~m}$. The motion on the inner and outer coil is plotted along with the approximation using Equation 5.2.
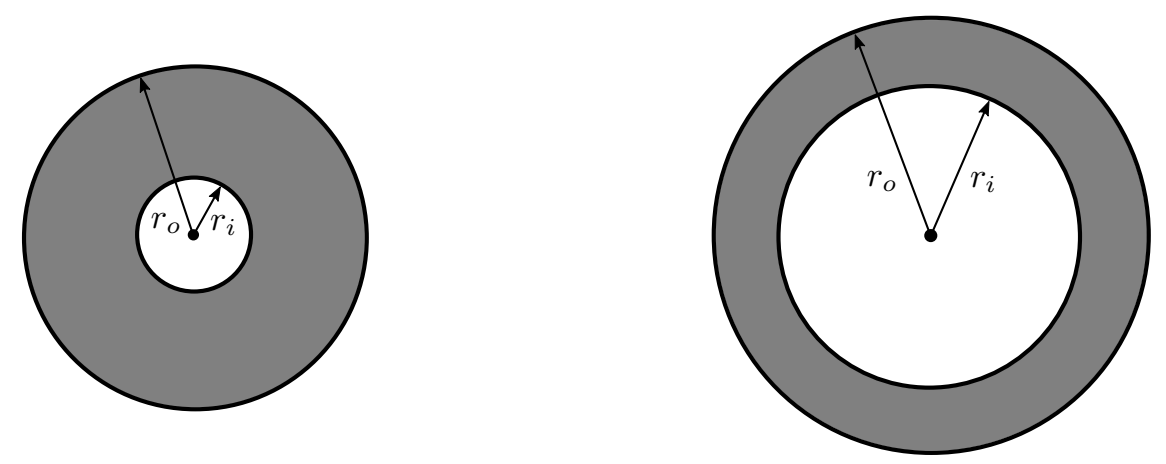

Figure 5.7: The changing of the inner coil radius $\left(r_{i}\right)$ and outer coil radius $\left(r_{o}\right)$ during blossoming. The inner coil radius increases faster than the outer coil radius due to overall thickness of the coil decreasing by one layer for each turn of the central spindle.

\subsection{Friction force}

When a coiled boom with compression rollers blossoms there will be a certain force from the compression rollers pushing the coil layers together. This compression force multiplied by the co- 
efficient of friction between the layers of the coiled boom will provide a certain force that has to be overcome before the coil layers can slide and blossoming can occur. If the relative motion is ignored this would be a simple scenario. Figure 5.8(a) shows the situation if the relative motion of the coil layers is ignored. A thin strip of material is fixed at one end and wrapped around a spindle. There are four compression rollers pushing against the outside of the thin strip of material. If a torque is applied to the spindle so that it slips and rotates, the tension force will be given by Equation 5.3 where $C_{f}$ is the coefficient of friction and $F_{c r}$ is the compression roller force. In this case the areas that cause the friction are directly under the compression roller and the contact areas in between the rollers are ignored. The spindle torque $\left(T_{s}\right)$ in this situation is the sum of the forces multiplied by the spindle radius $r_{s}$ as shown in Equation 5.4.

$$
\begin{gathered}
\text { tension }=4 F_{c r} C_{f} \\
T_{s}=4 F_{c r} C_{f} r_{s}
\end{gathered}
$$

Figure 5.8(b) shows a coiled boom that is about to undergo blossoming. In this case there are the same compression roller forces but the boom is attached to the central spindle and spindle turns in the opposite direction compared to Figure 5.8(a). Instead of creating a tension on the part of the boom that is deployed, the movement of the central spindle causes a compression. When the central spindle turns there are the same four contact areas, one under each compression roller, as shown in Figure 5.9. When blossoming occurs, the inside of the first layer of the coiled boom moves with the central spindle until a gap is formed so any contact between the central spindle and the first layer can be ignored. The outside of the first layer slides against the inside of the second layer. The relative motion between the outside of the first layer and the inside of the second layer depends on the number of coils and in this case there are two coils so the relative coil motion can be found using Equation 5.2. Equation 5.4 gives the torque in the situation in shown in Figure 5.8(a) and to change this equation to describe the situation in Figure 5.8(b) a modification to take into account the relative motion of the coils has to be made. The modification is the ratio between the amount of sliding that would occur in situation in Figure 5.8(a) and the amount of sliding in 5.8(b). In Figure 5.8(a) the relative motion between the strip of material and the central spindle for a complete turn of the central spindle would be given by $r_{s} 2 \pi$. In Figure $5.8(\mathrm{~b})$ the relative sliding motion for the average of inside and outside coil radii for a complete rotation can be found using Equations 3.16, 3.17 and 3.18. 


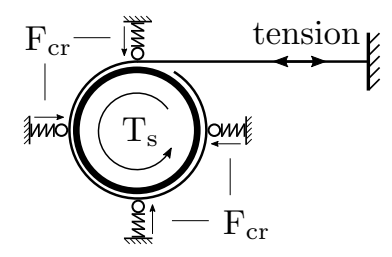

(a)

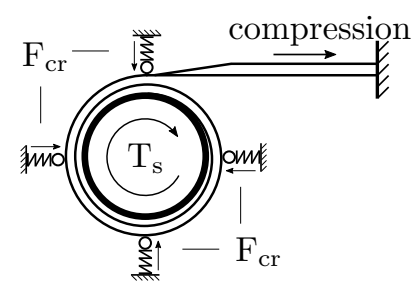

(b)

Figure 5.8: Comparison between a situation where the relative motion of the layers of coiled boom is not taken into account in (a) and where the relative motion is taken into account in (b).

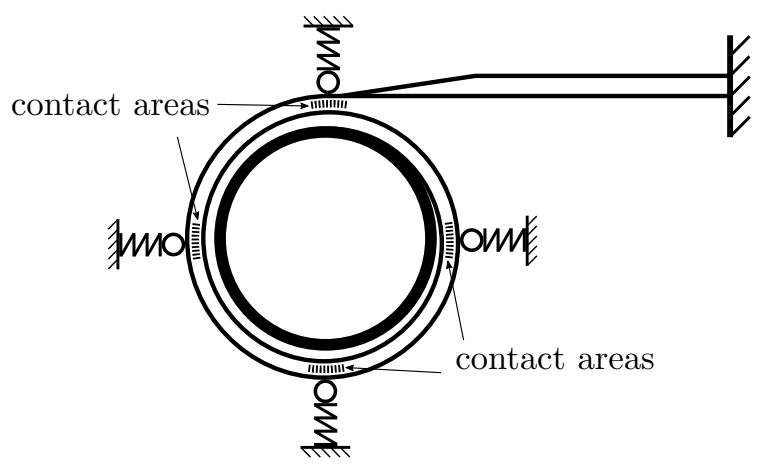

Figure 5.9: The contact areas under each compression roller.

A comparison between the amount of sliding that would happen if the relative sliding of the coil is taken in to account and the amount of sliding if the relative motion is not taken into account is shown in Figure 5.10. When the instantaneous distance slid not taking in to account the relative motion of the different layers is divided by the number of coils, the result is the distance slid, taking into account the number of coils. This relationship gives Equation 5.5 with the modification to take into account the relative motion by dividing by the number of coils $\left(N_{c}\right)$. The same compression roller force is assumed to act on all the coil layers. The coil radius is approximated to an average of the inner and outer radii. In this case the number of contact areas is dependant on the number of coils and to a first approximation is given by Equation 5.6. Initially there will be contact areas between the central spindle and the inside layer of the first coil, however the inside of the first coil and the central spindle move with the same relative motion until a gap forms so the contact between the central spindle and the first layer can be ignored. When the gap forms there will be 4 contact areas for each coil layer apart from the first layer, this gives Equation 5.6 where $N_{c a}$ is the number of contact areas and $N_{c r}$ is the number of compression rollers. 


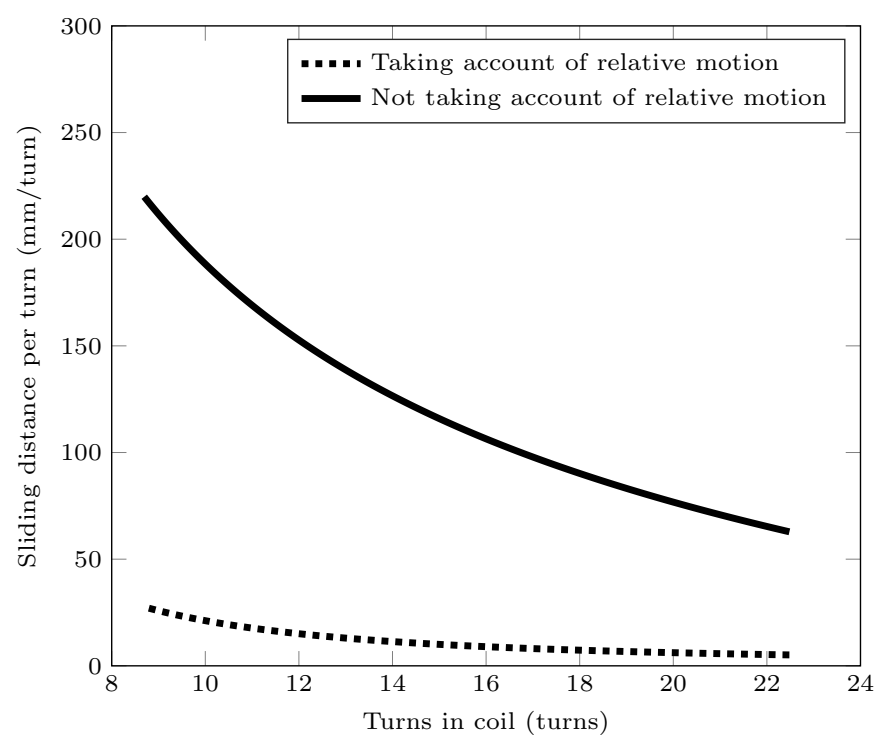

Figure 5.10: The difference in sliding distance taking into account the relative motion of the coil or not taking into account the relative motion of the coil.

$$
\begin{gathered}
T=N_{c a} F_{c r} C_{f} r_{s} \frac{1}{N_{c}} \\
N_{c a}=N_{c r} N_{c}-N_{c r}
\end{gathered}
$$

The relative coil motion has the effect of making the coils slide a lot smaller distance than they would if the relative motion was 1:1. This effect can be thought of as a gear ratio as is shown in Figure 5.11. A flat plate has a normal force applied to it and is being pulled across a table with a certain coefficient of friction. The tension needed to cause the plate to slide is given by the normal force multiplied by the coefficient of friction between the plate and the table. The cable that pulls the plate is attached to a spindle with a certain radius. Another part of that spindle with a larger radius has another cable attached, causing it to rotate. The force needed to cause the plate to move is the tension force divided by the ratio of the two spindle radii. This is an analogous situation to what occurs inside a coiled boom that is undergoing blossoming. The force needed to cause blossoming between the layers is given by the normal force multiplied by the coefficient of friction but due to the relative motion of the coils the torque that can be applied to a coil before it blossoms is decreased due to the gearing effect of the relative motion. This relative motion effect increases with the increasing number of coils. There is another effect that acts to counteract the decrease in potential coil torque that is caused by the friction between the coil layers as the number of coils increases. As the number of coils increases the number of contact areas also increases, this has the effect of increasing the amount of torque a coiled boom can withstand before blossoming. This can be thought of as another plate being added to the left of the current plate in Figure 5.11, being attached to the current plate and having its own normal force, leading to the tension increasing. These are two opposing mechanisms at work that almost cancel one another out. 


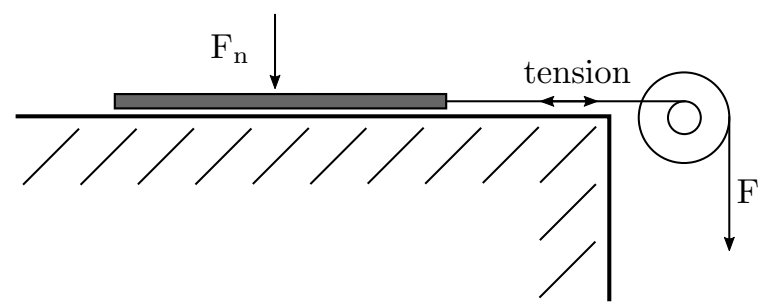

Figure 5.11: An analogy of the gear ratio effect of the movement in a coiled boom. The tension to cause a plate to slide across a surface while undergoing a normal force $\left(\mathrm{F}_{\mathrm{n}}\right)$ is given by the normal force multiplied by the coefficient of friction. The force required to turn the pulley is given by the tension divided by the gear ratio of the pulley.

\subsection{Results}

The following figures show the same theoretical and practical results shown in Chapter 4. Another line is added representing a central spindle torque taking into account the reduction caused by the friction between the coiled layers, using the theory developed above. A coefficient of friction between the coil layers is difficult to find practically as an unstressed tape spring will have a curved cross section so any attempt to slide two layers one on top of another with various normal forces applied would lead to difficulties and would not be representative of the contact experienced within a coiled boom. The coefficient of friction for two steel surfaces given in general literature varies in value between 0.1 and 0.7 depending on a variety of factors including the hardness and surface finish of the material, and whether the samples had any type of lubrication (Barrett, 1990; Oberg et al., 2016). It was decided to fit a reasonable approximation of the coefficient of friction to match practical results to see if the theoretical predictions stayed accurate over the range of compression spring forces and different number of turns in the coil. Initially Equations 5.5 and 5.6 were used to predict the central spindle torque including the friction. When a coefficient of friction of 0.4 was used the theoretical results seemed to have a greater negative torque compared to the practical results for the tests with a $1 \mathrm{~N}$ compression roller force. In the test cases with a $4 \mathrm{~N}$ compression roller force the theoretical results showed less of a negative torque than the practical results and the theoretical results in between followed a linear progression from too much negative torque to not enough. This suggested that the theoretical coefficient of friction was too high. A better match between the theory and the practical results was found by having an initial friction torque of 40 $\mathrm{Nmm}$ and a coefficient of friction of 0.25 . The $40 \mathrm{Nmm}$ is an initial friction torque caused by the rubbing together of coil layers that is present within a coiled boom without any compression roller force. This gave the results seen below in Figures 5.12 to 5.17. This seems to be a better approximation and correlates to the practical results for all the different compression roller forces. While this fitting of the coefficient of friction and an initial friction torque seems to have worked in this case more research would need to be carried out to verify that a coefficient of friction is accurate for the tape spring material used. This theory does not make any attempt to model the decrease and increase 'hump' in torque seen in the cases with boom type B which is thought to be caused by an initially tightly coiled boom slackening as blossoming occurs. While the theory to describe the friction within the coil presented seems to be a good starting point to model the reduced central spindle torque due to the friction, it seems that not all the aspects are modelled by it. The friction between the layers will depend on many variables such as any imperfections on the boom surface, any lubrication on the boom surface, whether the boom is in an atmosphere or in a vacuum and how tightly the coiled boom is wrapped. The decrease and increase of the practical results seen in the cases using boom type B show that the tightness of the coiled boom has a significant effect on the central spindle torque. If the application of a coiled boom is space related then the effect that violent vibrations during launch could have on the tightness and position of a 
coiled boom should be taken into account. While the theory above gives a good starting point to estimate the effect that the friction in the coil will have on the central spindle torque, it seems that the friction within a coiled boom is a complex phenomenon that is dependant on many factors and would be difficult to consistently predict with any great confidence.

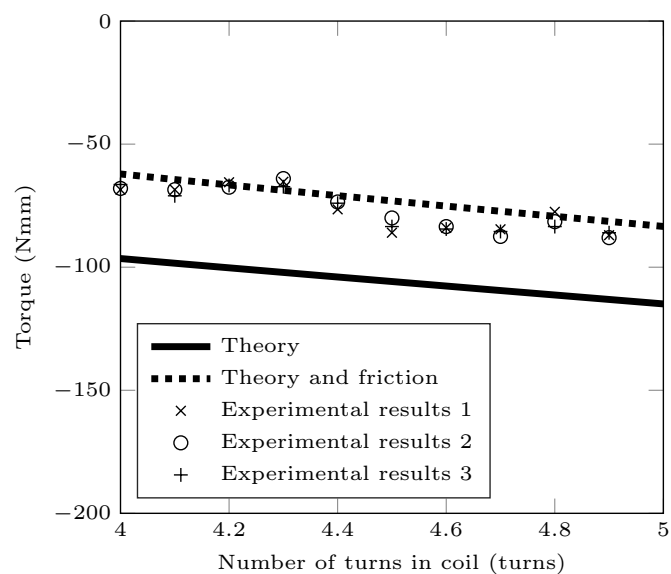

(a) $1 \mathrm{~N}$ compression spring force.

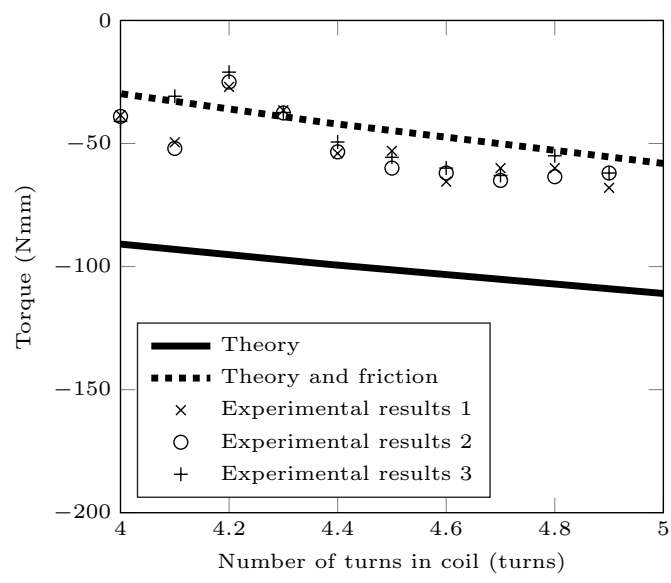

(c) $3 \mathrm{~N}$ compression spring force.

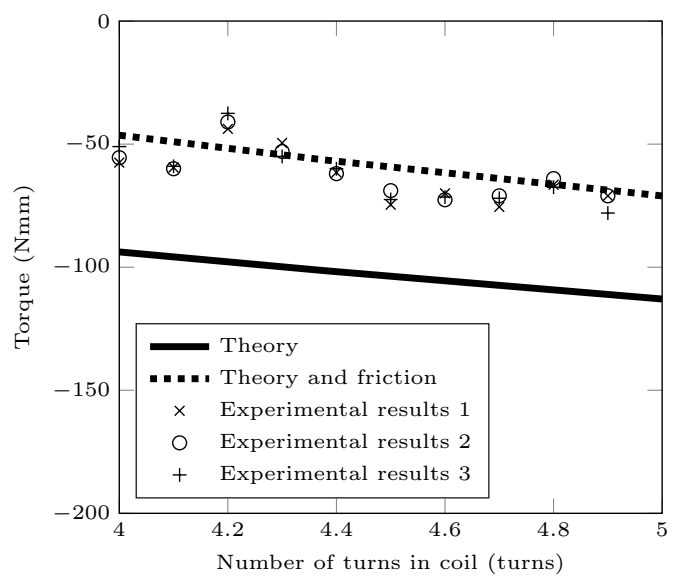

(b) $2 \mathrm{~N}$ compression spring force.

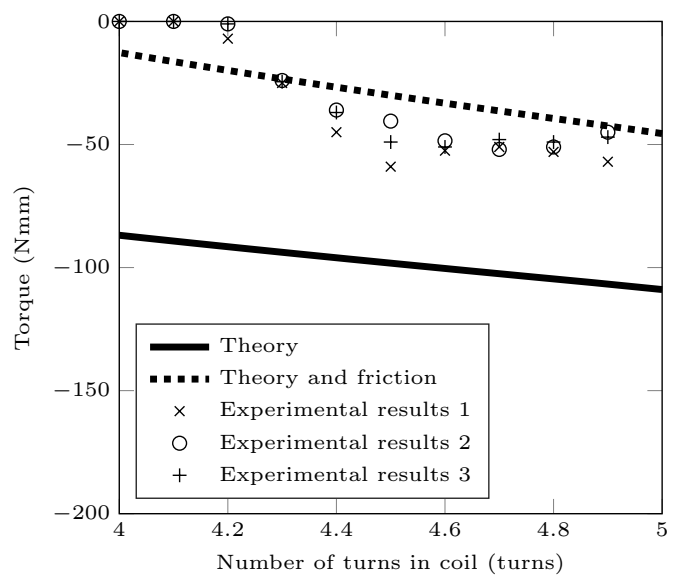

(d) $4 \mathrm{~N}$ compression spring force.

Figure 5.12: Results for boom type A, using a single boom and 5 coils. 


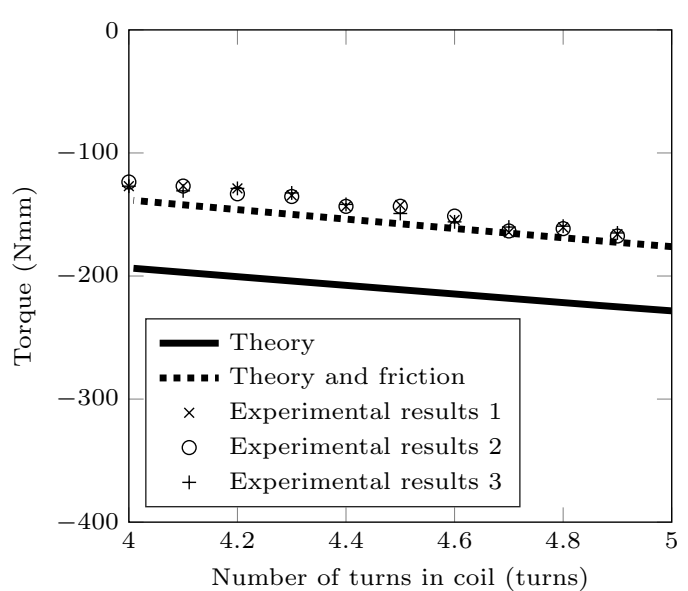

(a) $1 \mathrm{~N}$ compression spring force.

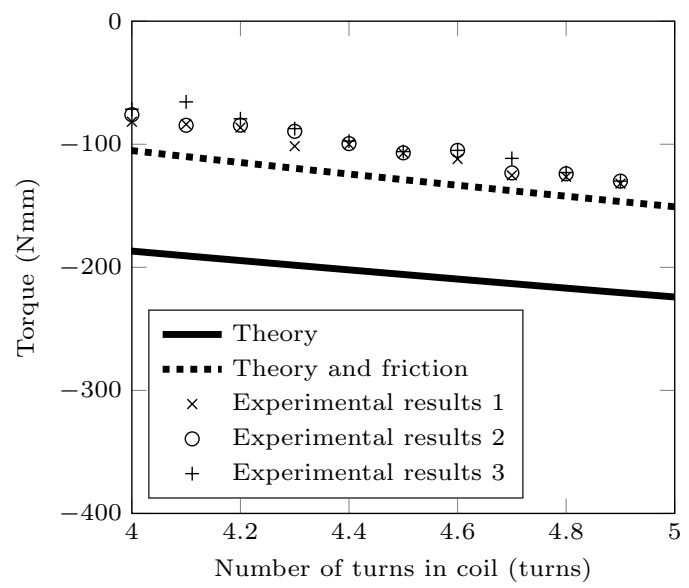

(c) $3 \mathrm{~N}$ compression spring force.

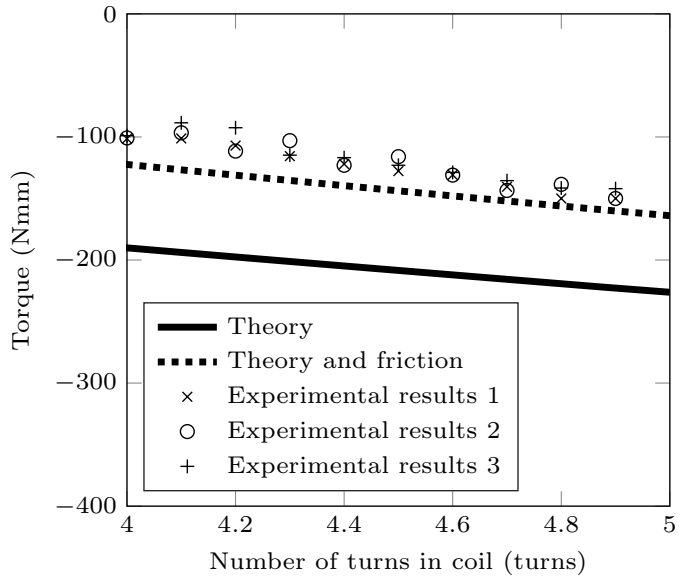

(b) $2 \mathrm{~N}$ compression spring force.

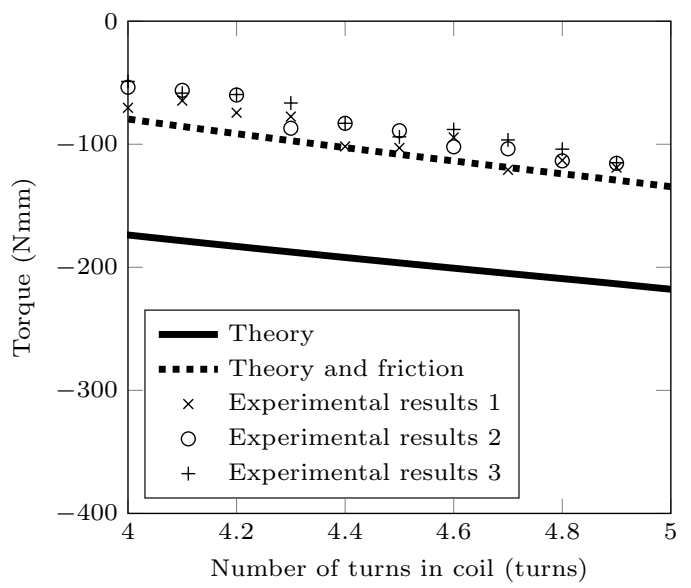

(d) $4 \mathrm{~N}$ compression spring force.

Figure 5.13: Results for boom type A, using two booms and 5 coils. 


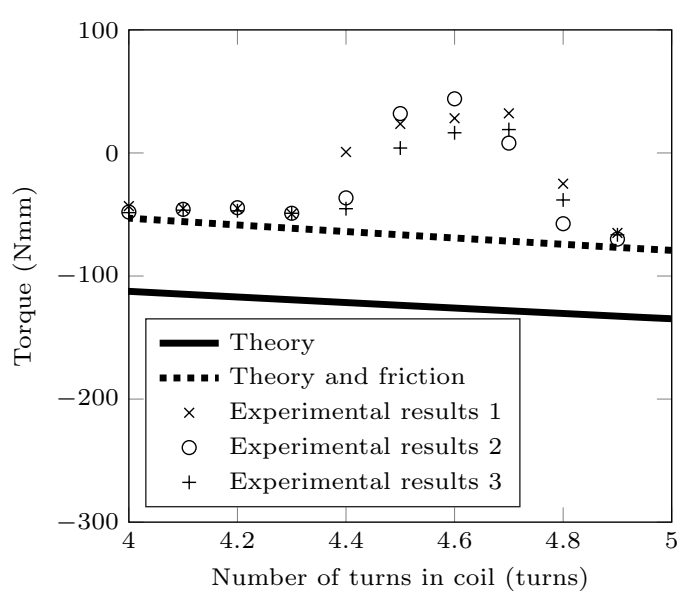

(a) $1 \mathrm{~N}$ compression spring force.

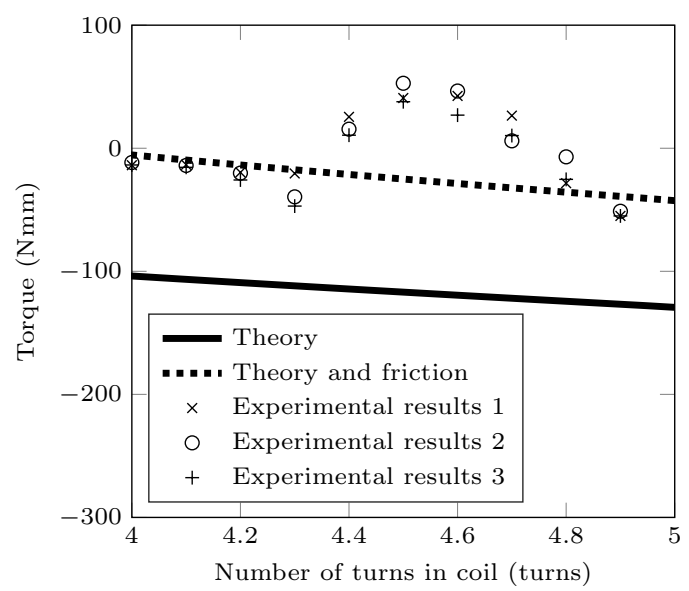

(c) $3 \mathrm{~N}$ compression spring force.

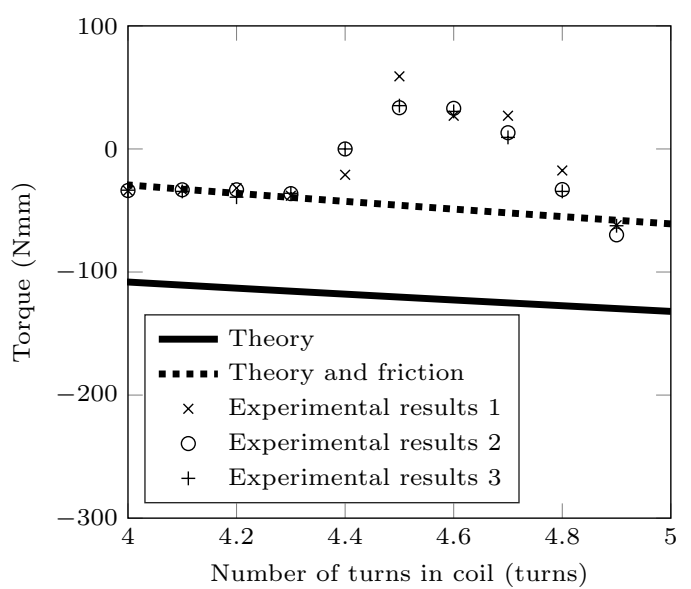

(b) $2 \mathrm{~N}$ compression spring force.

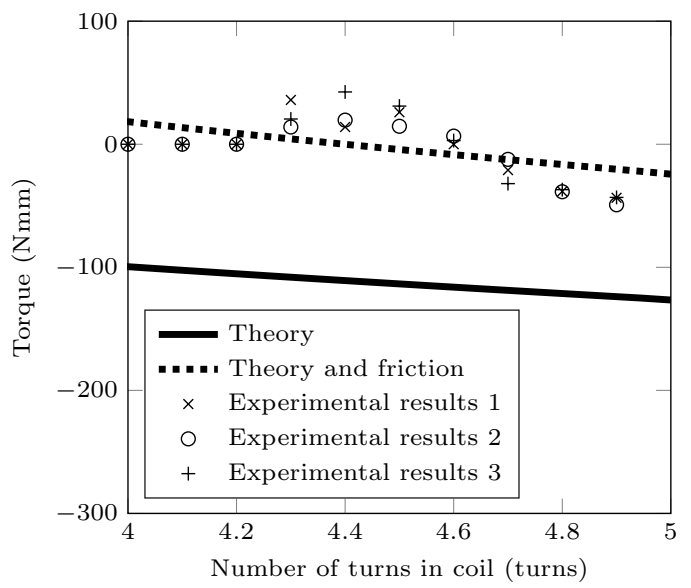

(d) $4 \mathrm{~N}$ compression spring force.

Figure 5.14: Results for boom type B, using two booms and 5 coils. 


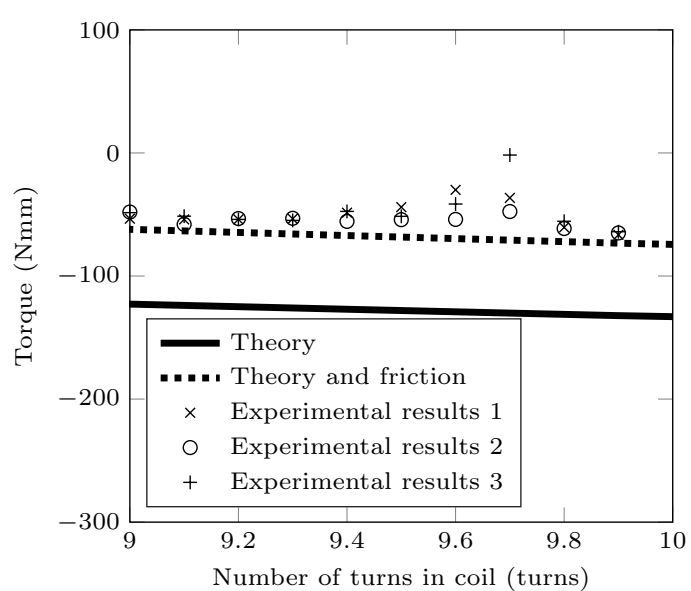

(a) $1 \mathrm{~N}$ compression spring force.

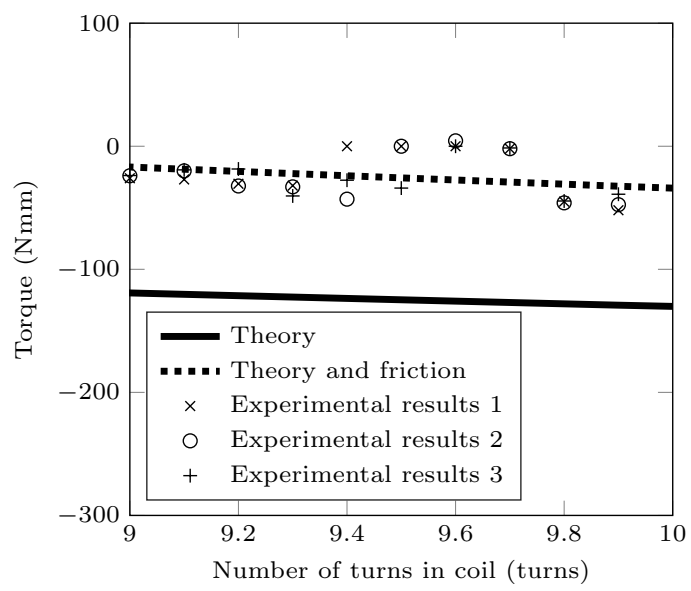

(c) $3 \mathrm{~N}$ compression spring force.

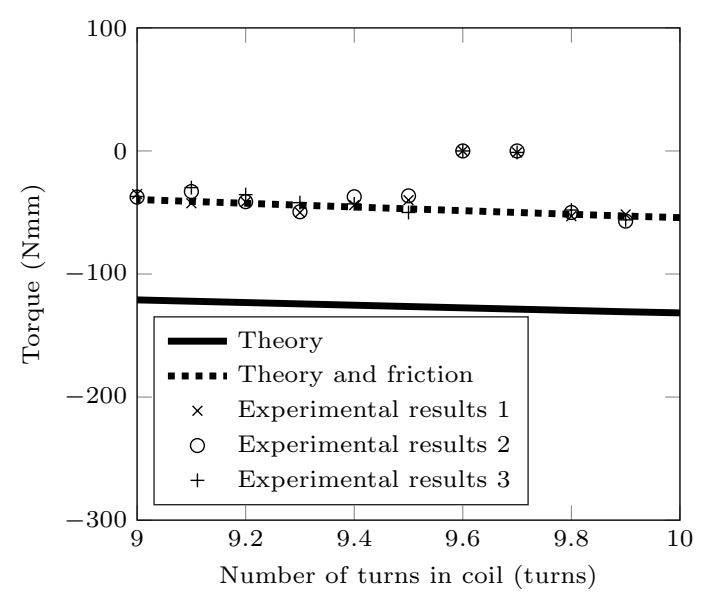

(b) $2 \mathrm{~N}$ compression spring force.

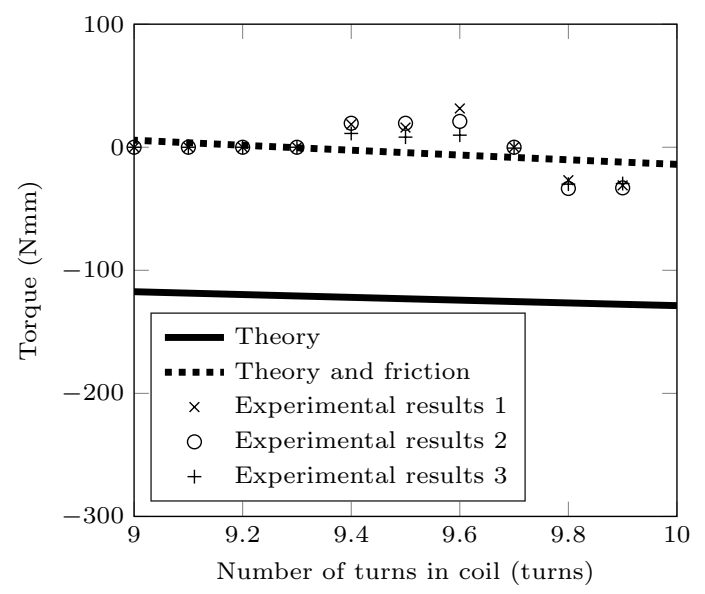

(d) $4 \mathrm{~N}$ compression spring force.

Figure 5.15: Results for boom type B, using two booms and 10 coils. 


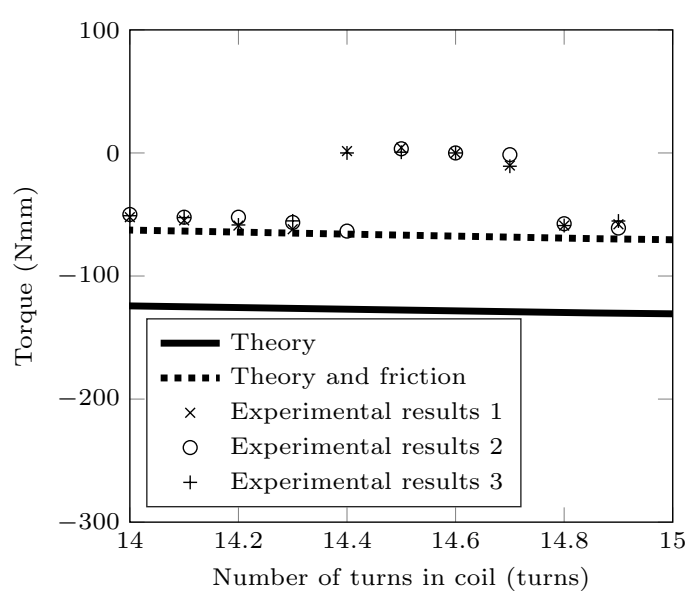

(a) $1 \mathrm{~N}$ compression spring force.

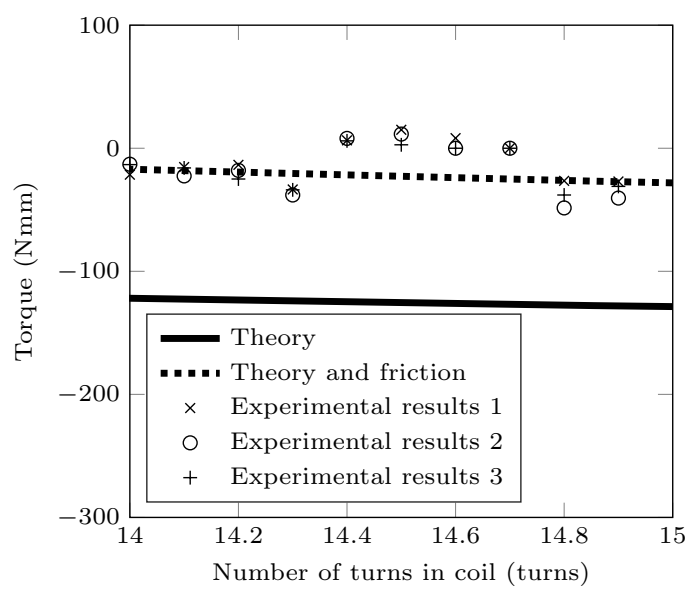

(c) $3 \mathrm{~N}$ compression spring force.

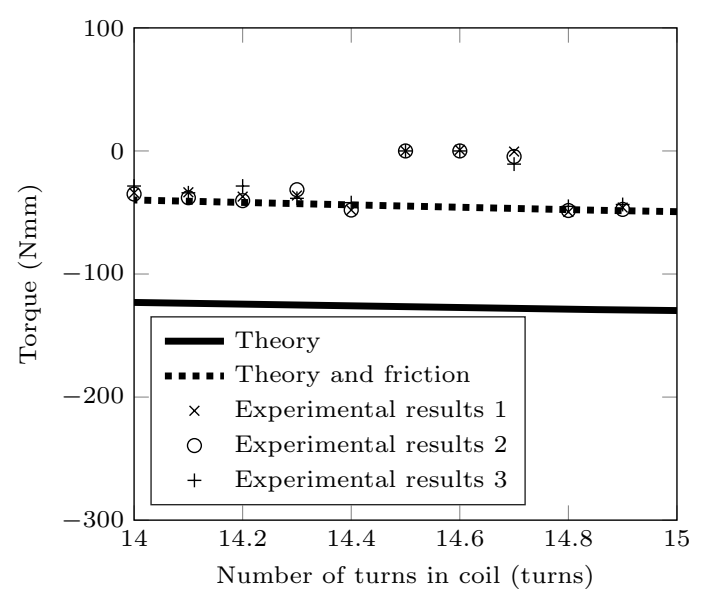

(b) $2 \mathrm{~N}$ compression spring force.

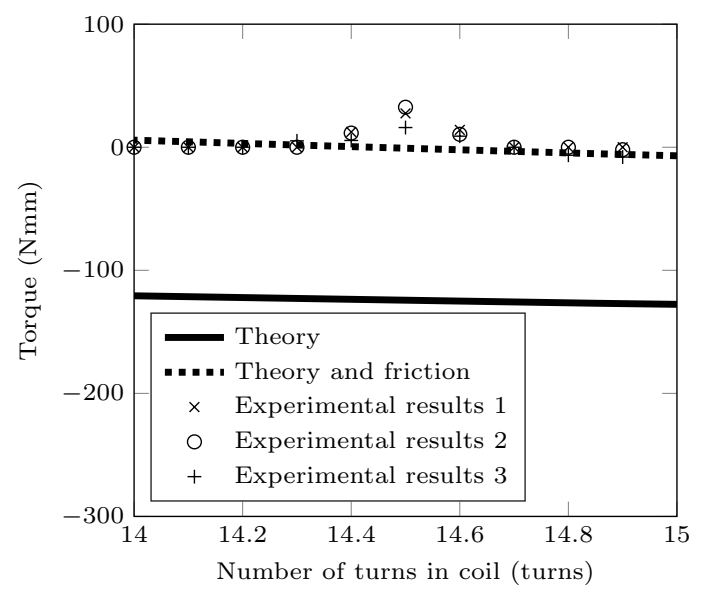

(d) $4 \mathrm{~N}$ compression spring force.

Figure 5.16: Results for boom type B, using two booms and 15 coils. 


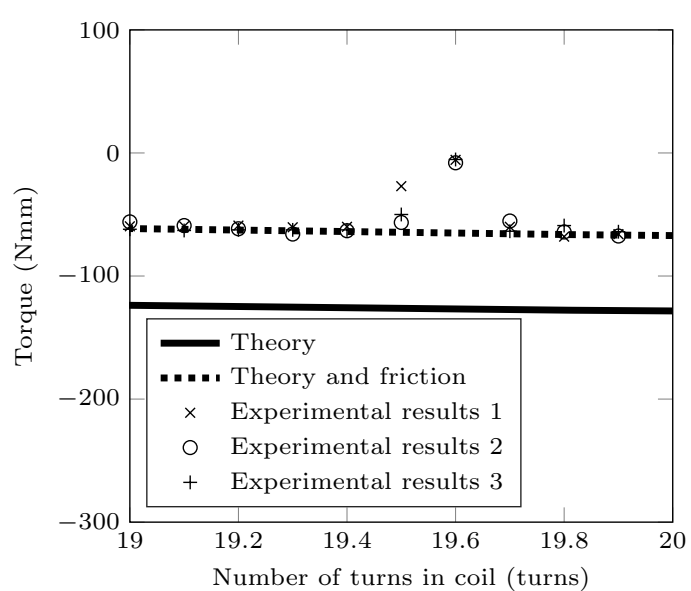

(a) $1 \mathrm{~N}$ compression spring force.

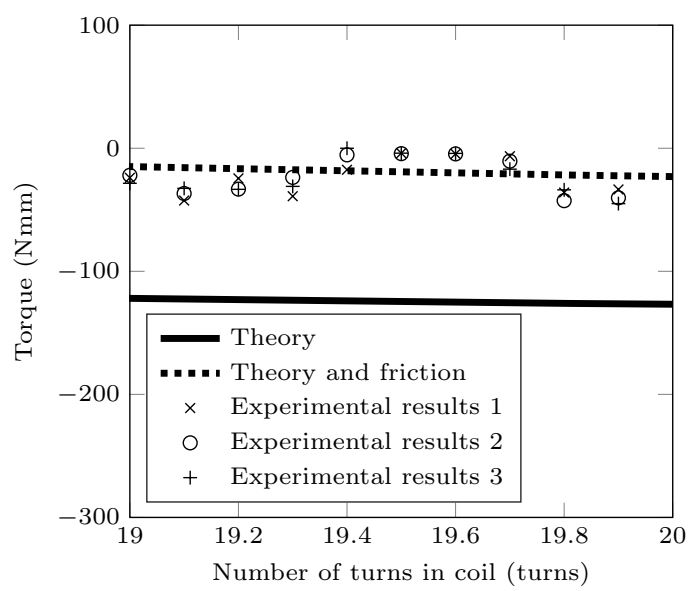

(c) $3 \mathrm{~N}$ compression spring force.

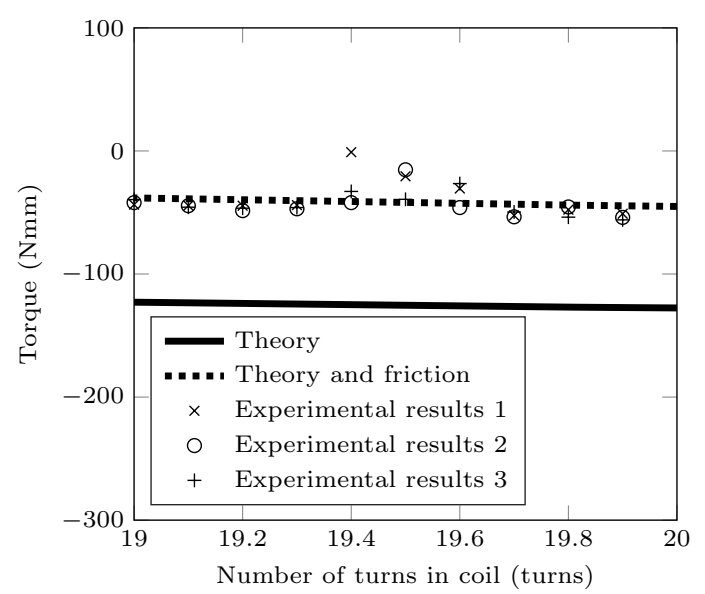

(b) $2 \mathrm{~N}$ compression spring force.

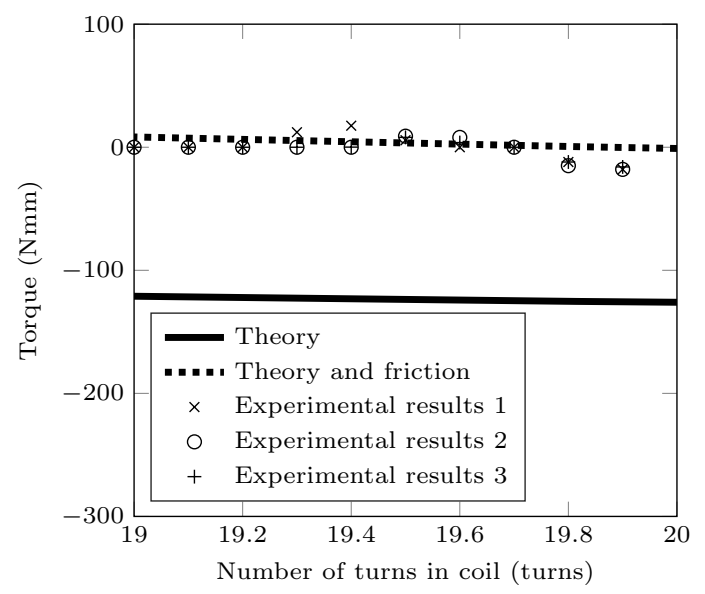

(d) $4 \mathrm{~N}$ compression spring force.

Figure 5.17: Results for boom type B, using two booms and 20 coils. 


\section{Chapter 6}

\section{Experimental test set-up}

This chapter details the design and operation of the experimental test set-up used for the experiments in the previous chapters. The test set-up has the ability to measure the central spindle torque and the tip forces. It also has the ability to turn the central spindle and alter the force applied by the compression rollers.

\subsection{Overview of experimental test set-up}

Figure 6.1 shows the whole experimental test set-up. The deployer is the main part of the experimental set-up where the booms are coiled around the central spindle. The motor and torque sensors are also housed within the deployer along with the compression roller arms. The frame which is seated on top of the deployer holds the tip force sensors at a distance away from the booms. The experimental test set-up has two modes of operation. The experiments detailed in this thesis have been carried out with the boom tips constrained to the force sensors and are therefore held stationary while blossoming occurs. The test set-up has another operation mode where instead of holding the tips stationary, the booms can deploy while overcoming a constant force. This is achieved by attaching a thin cord from the boom tips to weights hanging from the frame. The thin cord runs from the boom tip towards the base, through $90^{\circ}$ around a pulley so it becomes vertical. It then runs around another pulley at the top of the frame and from it hangs a weight. This enables a constant force to be applied during deployment of the boom and is part of the reason for the frame.

Figure 6.2 shows the deployer with the compression arms and the pulley that turns the thin cord from parallel to the boom to vertical to go to the tip force sensor mounted on the frame. The motor and the torque sensor can also be seen. 


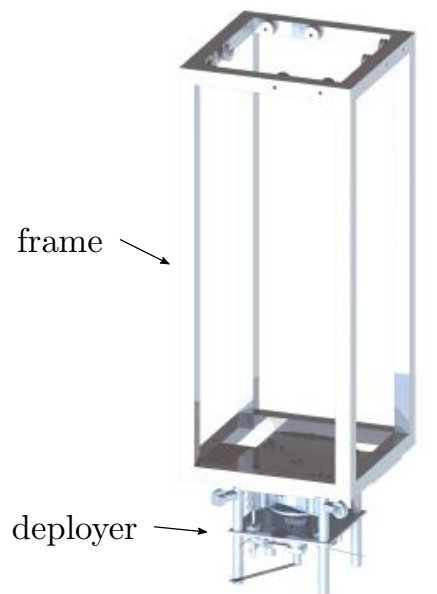

Figure 6.1: The experimental test setup with the deployer and the frame.

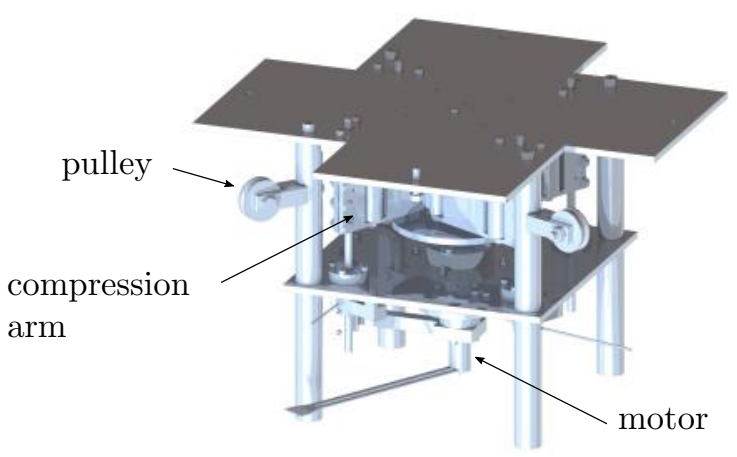

Figure 6.2: The deployer part of the experimental test set-up.

\subsubsection{Central spindle}

The boom roots are connected to the central spindle and as the spindle is turned the boom coil forms around it. The central spindle has a bearing at the top and bottom of the shaft and a slot that connects via a drive shaft to the output of the motor gearbox, as shown in Figure 6.4. The motor has the ability to rotate the central spindle relative to the rest of the test set-up. Each boom is connected via two M4 button head bolts, this flattens the boom cross section at the root and aligns the direction of the boom at $90^{\circ}$ to the spindle axis. As the central spindle is turned and the booms coil, the recess that has been cut for the bolt heads allow the booms to form a circular coil around the central spindle as shown in Figure 6.3.
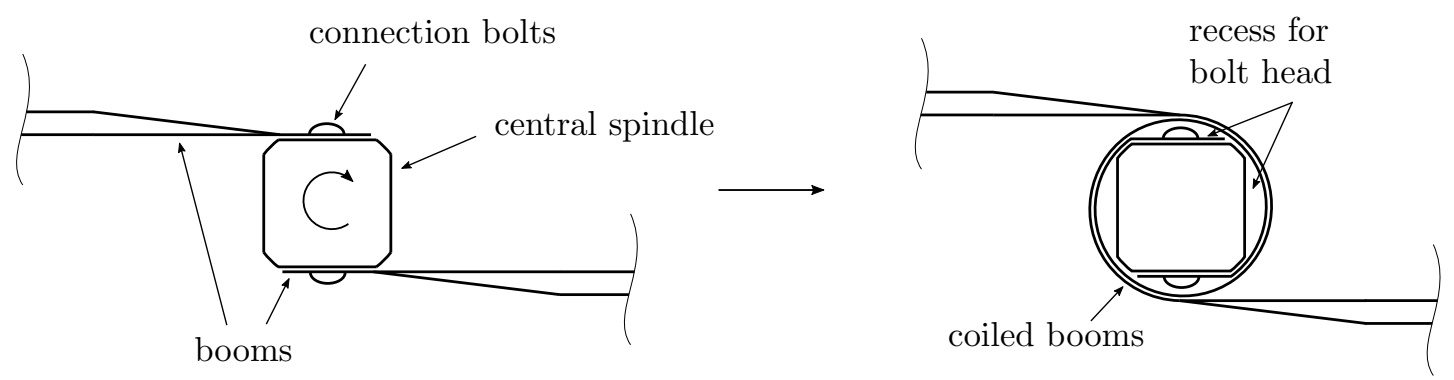

Figure 6.3: Top view of the central spindle. The process of the boom attachment and coiling is shown. 


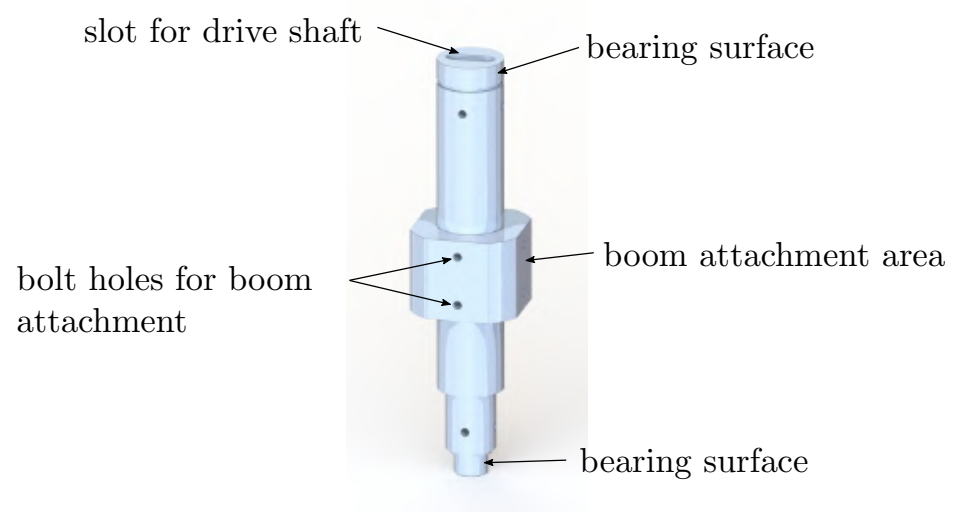

Figure 6.4: Side view of the central spindle. The boom attachment points and the slot for the drive shaft are shown.

\subsubsection{Central spindle torque sensor and motor}

The central spindle is connected to the motor gearbox via a drive shaft, as shown in Figure 6.5. The motor is mounted inside a motor case which is constrained by a bearing attached to the rest of the test set-up. This allows the motor case and motor to rotate within the bearing. The only thing that is stopping the motor casing from rotating is the torque arm, therefore all the central spindle torque is resolved through the torque arm and onto the force sensors. By measuring the force on the force sensors and multiplying by the distance between the tip of the torque arm the centre of the rotation point of the motor casing, the central spindle torque can be determined. This principle is shown in Figure 6.6. The force sensors used were Honeywell FSS series low profile force sensors (FSS015WNST). These give a voltage proportional to the force applied. The data from the sensors was recorded using a NI-9205 voltage input module and CompactDAQ using LABVIEW software. The sensors were calibrated by hanging known weights to give a force voltage gradient.

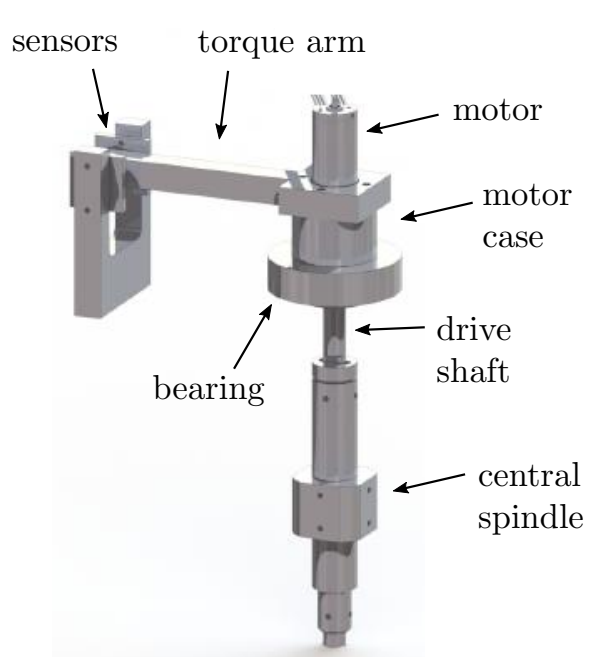

Figure 6.5: The parts that make up the central spindle torque measurement.

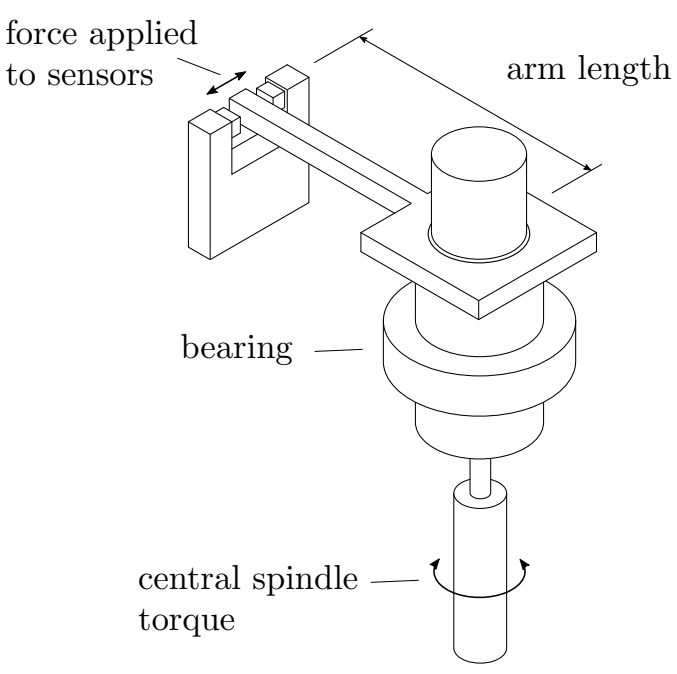

Figure 6.6: The central spindle torque is found using the force applied to the sensors and the length of torque arm.

\subsubsection{Tip force sensors}

To measure the tip force of a boom during blossoming a thin chord was attached between the boom tip and the force sensor. The thin chord runs parallel to the boom, it then rotates through 
$90^{\circ}$ around a pulley and becomes vertical as shown in Figure 6.2. The thin chord then attaches to the tip force sensor at the top of the frame. The tip force sensors are cantilever beams with strain gauges attached on their top surfaces as shown in Figure 6.7. The thin chord is attached via a bolt that goes through the tip of the cantilever beam. This allows for a small amount of adjustment necessary during the testing process as the booms do not always come back to the same place after each test due to the nature of the coiling process. A nut is locked against the bolt to keep it in the correct position. The tip force sensors were calibrated by hanging weights from the bolt via a separate thin chord. Once it was established that there was a linear relationship between force and strain over the range of forces that the booms might apply to the force sensors a single mass was tested after each set of tests to verify that the sensors were working correctly. A National Instruments strain gauge input module NI-9237 and a CompactDAQ was used to record the strain during the experiments using LABVIEW software.

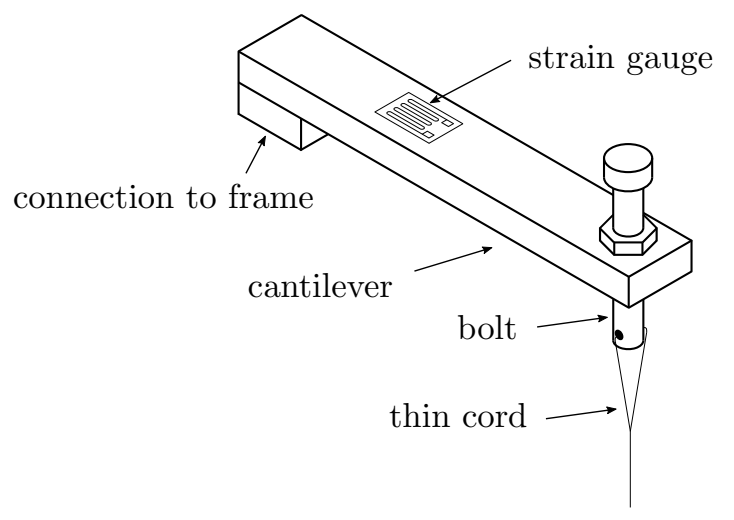

Figure 6.7: Tip force sensor. The root of the sensor attaches to the frame. The tip of the sensor is attached to the boom tip via a thin chord.

\subsubsection{Compression roller arms}

A compression arm is shown in Figure 6.8. The arm has one compression roller that compresses the boom coil and another guide roller that guides the boom as it exits the deployer. The arm is connected to a shaft that is held in place using two bearings, one at the top and one at the bottom of the deployer. This allows the whole arm to rotate freely about the shaft. A torque is applied to the shaft and hence the compression arm by the torsion spring. One leg of the torsion spring is constrained to the deployer floor and the other end is constrained by the torsion spring arm. The top part of the shaft is threaded. The torsion spring arm is also threaded which allows its angle relative to the shaft to be adjusted. Once the correct angle is achieved it can be fixed in place with a locking nut. This allows the angle of the torsion spring and hence the compression roller force to be adjusted. Figures 6.9 shows a side view of the four compression arms around the central spindle and Figure 6.10 shows a top view. 


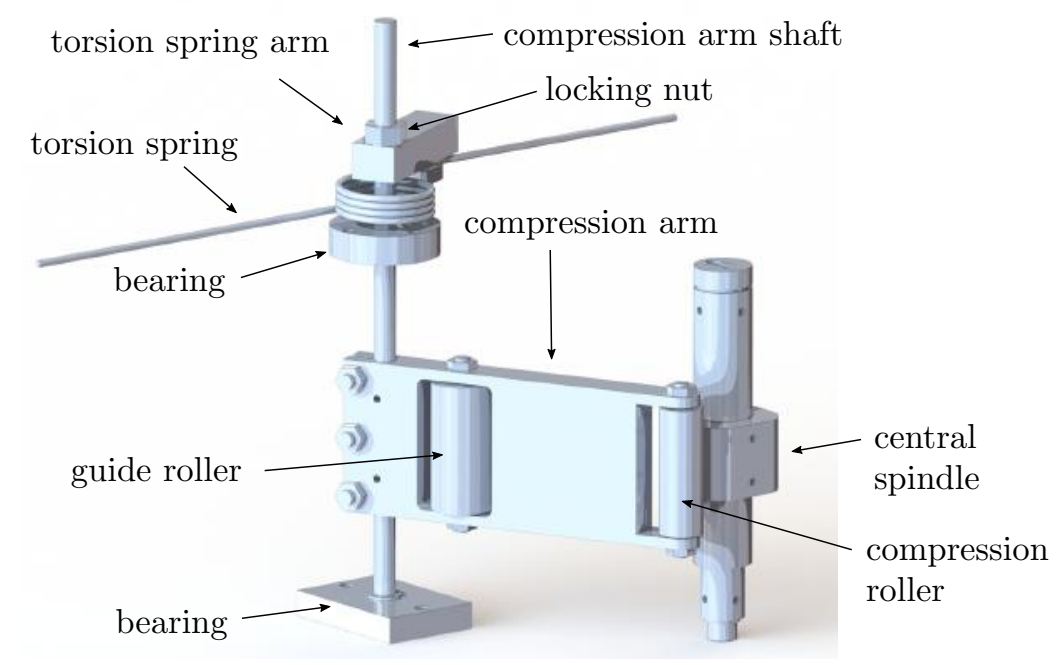

Figure 6.8: A single compression arm assembly with the central spindle.

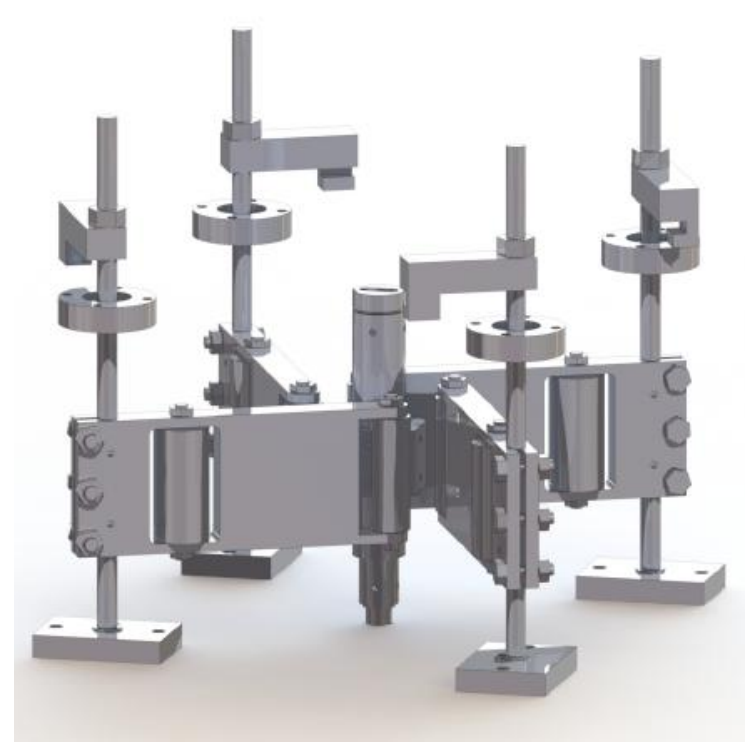

Figure 6.9: A view of the four compression arm assemblies around the central spindle.

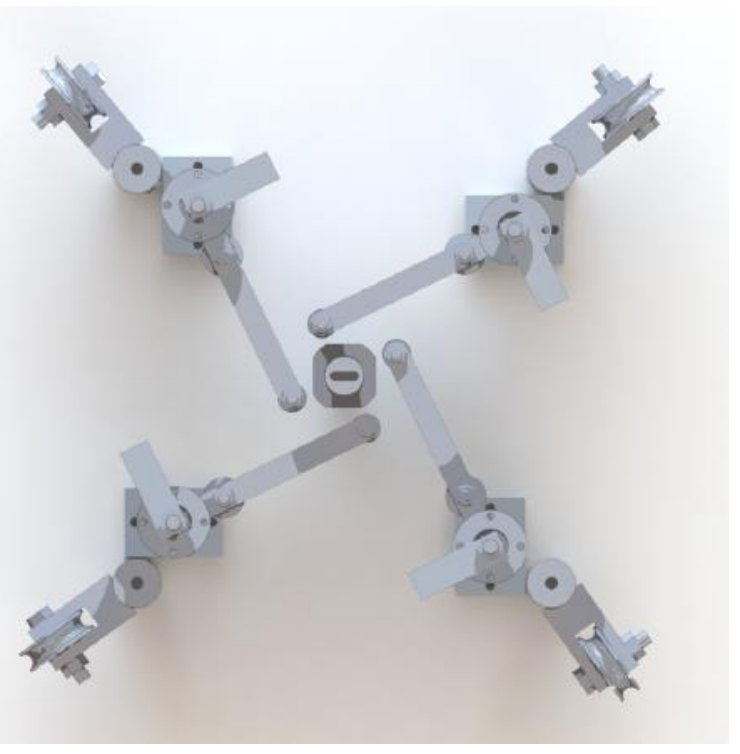

Figure 6.10: A top view of the four compression roller assemblies and the central spindle.

\subsection{Details of experimental method}

\subsubsection{The process}

To carry out the experiment the correct number of booms were attached to the central spindle and the central spindle was turned until the boom coil had the correct number of turns. The clamps that attached the thin chord to the boom tips were adjusted to the correct length so that the chord had no slack. The tension of the thread was adjusted by turning the bolt on the force sensor until the tip force was zeroed. The compression roller arm springs had their angle adjusted until the correct compression roller force was achieved. This was measured by using a force meter connected to the compression roller. The central spindle was then turned in increments of $36^{\circ}$ using the electric motor. During this time the tip force and central spindle torque was recorded. This was continued for the the correct number of rotations of the central spindle. The central spindle was then rotated in the opposite direction until the original position of the booms was reached. 
The process was repeated three times for each set of parameters.

\subsubsection{Data collection}

During the experiments LABVIEW was used to record the strain measurements from the tip force sensors and the force from the sensors for the central spindle torque. This data could then be manually processed to give tip forces and a central spindle torque corresponding to each angle of rotation of the central spindle. The tip force sensors and the force sensors used for the central spindle torque were calibrated and checked using known weights. These weights were weighed using calibrated scales with an accuracy of $\pm 0.001 \mathrm{~N}$. When LABVIEW recorded the data from the force sensors used to find the central spindle torque and the strain gauges used to find the tip force it did so at a resolution of the equivalent of $\pm 0.00001 \mathrm{~N}$. Due to the signal noise and vibrations the accuracy of the reading should be taken to be $\pm 0.01 \mathrm{~N}$ for the tip force sensors and $\pm 0.001 \mathrm{Nm}$ for the torque sensors. The force meter used to measure the compression roller force had an accuracy of $\pm 0.2 \mathrm{~N}$. 


\section{Chapter 7}

\section{Conclusion}

In recent years there has been a renewed interest in large deployable structures for space and especially for use in solar sailing and as drag deorbiting devices. There have been a number of similar solar sailing or drag deorbiting missions that use four booms to deploy and tension four triangular sails to give a square form sail. During deployment testing in some of these missions blossoming occurred. There seems to be very little information in the literature to describe the blossoming problem or to predict under what loads or conditions it will occur. The work carried out that has been presented in this thesis has been aimed at investigating the blossoming problem in an attempt to give future deployer designers tools to use during the design process to predict the possible tip forces and central spindle torques that are likely to be experienced before and during blossoming.

The work carried out breaks down into three main parts: a method to find the tip force, a method to find the central spindle torque and an investigation into the motion between each layer of the boom within the boom coil.

\section{Tip force}

A method using the strain energy stored in the coiled part of the boom was used to find the tip force. An initial model simplified the spiral geometry of the coil to a constant radius allowing a first approximation of the tip force to be found. Experiments without compression rollers demonstrated that the method showed convincing correlation between the theory and practical results. The model was developed to take into account the spiral geometry of the coiled boom and the strain energy stored in the compression springs. A more elaborate experimental test set-up was created that could measure the tip force and the central spindle torque. The central spindle was turned in increments while the tips of the booms were held stationary to cause blossoming to occur. The central spindle torque and tip forces were recorded for two different booms and for a range of compression roller forces and number of turns in the coil. The results from these tests were compared to the theoretical predictions. In most cases with a small compression roller force the practical tip forces were fairly accurately predicted by the theory. As the compression roller forces increased the practical results displayed the same gradient of tip force compared to the theory but the practical forces were higher than the theory suggested. This is thought to be due to the increase in friction between the coil layers as the compression roller force was increased. For blossoming to occur the coil layers have to slide past one another, if the compression roller force is increased it will make it more difficult for the coil layers to slide past one another, increasing the tip force. The strain energy method does not attempt to include the effects that the friction in the coil has on the tip force but can be used as a good first approximation to determine how much tip force a coiled boom with compression springs can resist before blossoming. The friction 
in the coil will always increase the tip force above that predicted by the strain energy method so the strain energy theory could be used as a minimum expected tip force. This would possibly be somewhat conservative but as the amount of extra tip force that could be expected due to the friction within the coil is hard to model and is highly sensitive on a number of parameters it may be a good starting point for deployer designers.

\section{Central spindle torque and self-deployment torque}

A similar strain energy method was used to model the central spindle torque and self-deployment torque of a coiled boom. The self-deployment torque originates from the reduction in strain energy that occurs when the tape spring changes from a flat cross section to a curved cross section on the outside of the coil. This creates a torque that attempts to uncoil the tape spring and unless the central spindle is stopped from turning the boom will self-deploy. The self-deployment torque is at a minimum when the coil radius is the same as the tape spring's natural radius, if the coil radius is above or below the natural radius the self-deployment torque will increase.

The central spindle torque originates from the reduction in strain energy if the outside of the coil is kept stationary and the central spindle is allowed to turn. The turning of the central spindle will reduce the number of turns in the coil and also increase the coil's radius, decreasing the strain energy. This leads to a trend of the central spindle torque decreasing as the central spindle turns and the coil increases in size. When the coil is at the tape spring's natural radius the central spindle torque and the self deployment torque will be equal in magnitude. This occurs when the central spindle is held fixed and the outside of the boom has a section that is deployed but the deployed part of the boom is stopped from uncoiling by the guide roller. In this situation when a tip force is applied towards the deployer the coiled boom will increase in diameter, this will increase the self-deployment torque and decrease the central spindle torque. This gives a force moment balance with the central spindle torque, the self deployment torque and the tip force.

During the practical experiments used to test the tip force theory the central spindle torque was also recorded. The theoretical central spindle torque in all cases was negative, meaning that the coiled boom was trying to uncoil but the central spindle was stopping it from doing so. As blossoming occurred the central spindle torque decreased as the tip force increased. In all the cases the practical results followed this trend of a decrease in negative torque as blossoming occurred. While the practical results followed the similar gradients of decreasing central spindle torque as blossoming occurred, the practical results showed significantly less negative torque compared to the theory. The negative torque decreased further as the compression roller force increased. The smaller negative torque and the further decrease in negative torque as the compression roller force was increased shown in the practical results were caused by the friction within the coiled booms which increased as the compression roller force was increased. The important discovery that came from investigating the central spindle torque was that the deployment of the coiled boom and the tip force originated mostly from the strain energy stored in the coiled boom and not from the turning of the central spindle. In most of the experimental cases the central spindle was stopping the inside of the coil from deploying as opposed to driving the deployment of the boom. This may change if much larger compression roller forces or booms with a significantly higher coefficient of friction are used.

\section{Coil motion}

Finally an attempt to understand the nature of the motion of the boom within the coil was made and how this effected the friction within the coil. When a coiled boom blossoms the friction be- 
tween each layer must be overcome and the coil layers must slide past one another. The relative motion depends on the length and number of turns in the coil. When there is only just over one turn in the coil the relative motion between the outside of the first layer and the inside of the start of the second layer is the same as the motion between the central spindle and a fixed position. As the number of coils increases the relative motion between each coil layer decreases significantly. This motion has the effect of reducing the amount of torque a coiled boom can resist before blossoming and has the same effect as the sliding motion being driven through a gear ratio. Another effect of increasing the number of turns in the coil is to increase the number of contact points that the coiled boom has. These two effects almost cancel each other out. This theory was fitted to the practical results giving a coefficient of 0.25 and an initial central spindle torque of $40 \mathrm{Nmm}$. The initial central spindle torque originates from the inherent friction within a coiled boom that comes from the rubbing together of the coil layers without any compression roller force. While this theory consistently matched the results with different compression roller forces and different numbers of coiled booms, more work would need to be carried out to verify the coefficient of friction of the boom. Although the theory predicted the general trend of the central spindle torque, it made no attempt to describe the 'humped' decrease and increase in negative central spindle torque seen in the cases using boom B. The decrease and increase in torque was thought to be caused by the initial tightness of the coiled boom which loosened during blossoming. The effect of the initial tightness of the coiled boom which caused the 'humping' effect shows the inconsistent nature of blossoming and of the friction in the coil. The amount the tip force increases above the force predicted by the theory or the amount the central spindle torque reduces below what the theory predicts can be inconsistent due to the inconsistent nature of the friction within the coil. The friction can be changed by a variety of factors including: the surface finish of the boom, the tightness of the winding process of the coiled boom, any lubrication of the boom surface, any irregularities of the boom surface or any grit or dust. Another factor that will effect the coefficient of friction will be if the coiled boom is in a vacuum or not. When in an atmosphere two surfaces usually have a small air gap between one another whereas when in space metals can cold weld together, increasing the coefficient of friction further. The only way to consistently know the effect that the coefficient of friction will have on the tip force and central spindle torque is repeated testing, however the initial theory should provide a starting point to aid predictions.

It is hoped that this work has resulted in a greater understanding of the mechanics of coiled boom deployers, the deployment process, and blossoming. The energy method should allow deployer designers to calculate the tip force and central spindle torque a coiled boom with certain parameters will display. This method should be used as a tool in the design process and hopefully eliminate unforeseen blossoming issues that have arisen during the deployment testing phase of projects.

\section{Future work}

The strain energy method used to find the tip force, the central spindle torque and self-deployment torque was developed and tested for the case of simple tape spring booms due to the simplicity of calculating the strain energy with this boom type. While some tape spring and STEM type booms are still used for various applications, different boom cross sections and materials are also being used. To enable the methods presented to be used with different boom cross sections and with booms made from different materials such as fibre reinforced plastics, work would have to be carried out investigating the strain energy of these different booms types when coiled. It is thought that the strain energy minimum that is displayed by a tape spring when the boom is held in a coil with a constant subtended angle would also be displayed by a TRAC boom and DLR's lenticular 
boom when using isotropic materials. However the TRAC and lenticular booms have two halves that are only joined together in certain parts of the cross section which may cause some differences in behaviour. Other booms that use fibre reinforced plastics such as the bistable over the whole length (BOWL) boom will need a different and more complex method to find the strain energy at different coil radii. For both these cases, once the strain energy is found for the booms held at different coil radii, the same method of finding the change in strain energy for a given length change or change in strain energy for a given angle turned by the central spindle can be carried out. This gives the tip force and central spindle torque. 


\section{Bibliography}

Abercrombie, R.A. Torsional measurements of satellite booms. NASA Technical Note, 1971.

Alhorn, Dean, Casas, Joseph, Agasid, Elwood, Adams, Charles, Laue, Greg, Kitts, Christopher, and OBrien, Sue. Nanosail-d: The small satellite that could! 26th Annual AIAA/USU Conference on Small Satellites, 2011.

Banik, Jeremy and Murphey, Thomas. Performance validation of the triangular rollable and collapsible mast. 24th Annual AIAA/USU Conference on Small Satellites, 2010.

Barrett, Richard T. Fastener design manual. National Aeronautics and Space Administration, Office of Management, Scientific and Technical Information Devision, 1990.

Biddy, Chris and Svitek, Tomas. Lightsail-1 solar sail design and qualification. In Proceedings of the 41st Aerospace Mechanisms Symposium, 2012.

Blandino, Joseph R, Johnston, John D, and Dharamsi, Urmil K. Corner wrinkling of a square membrane due to symmetric mechanical loads. Journal of Spacecraft and Rockets, 39(5):717$724,2002$.

Blomquist, R. Solar blade nanosatellite development: Heliogyro deployment, dynamics, and control. In Proceedings of the 13th USU/AIAA Small Satellite Conference, 1999.

Bourgeois-Doyle, Richard I. George J. Klein: The Great Inventor. NRC Research Press, 2004.

Calladine, CR. The theory of thin shell structures 1888-1988. Proceedings of the Institution of Mechanical Engineers, Part A: Journal of Power and Energy, 202(3):141-149, 1988.

Calladine, CR. Theory of shell structures. Cambridge University Press, 1989.

Documentation, Matlab. The mathworks inc, 2005.

E Roberts, Peter C and Harkness, Patrick G. Drag sail for end-of-life disposal from low earth orbit. Journal of Spacecraft and Rockets, 44(6):1195-1203, 2007.

Elliott, T and Rimrott, FPJ. Torsion of slit, overlapped, thin-walled tubes. Journal of Spacecraft and Rockets, 3(6):873-876, 1966.

Farrand, Hiram A. Rule, January 3 1922. US Patent 1,402,589.

Farrand, Hiram A. Resilient rule, October 20 1931. US Patent 1,828,401.

Fellows, Alvin J. Improvement of tape measures, July 14 1868. US Patent 79,965.

Fernandez, J, Schenk, M, Prassinos, G, Lappas, VJ, and Erb, SO. Deployment mechanisms of a gossamer satellite deorbiter. In 15th European Space Mechanisms and Tribology Symposium, Noordwijk, The Netherlands, 2013. 
Fernandez, Juan M, Viquerat, Andrew, Lappas, Vaios J, and Daton-Lovett, Andrew J. Bistable over the whole length (bowl) cfrp booms for solar sails. In Advances in Solar Sailing, pages 609-628. Springer, 2014.

Fischer, Annette. Bending instabilities of thin-walled transversely curved metallic strips. University of Cambridge, Department of Engineering, 1995.

Galletly, Diana A. and Guest, Simon D. Bistable composite slit tubes. i. a beam model. International Journal of Solids and Structures, 41:4517 - 4533, 2004a.

Galletly, Diana A and Guest, Simon D. Bistable composite slit tubes. ii. a shell model. International Journal of Solids and Structures, 41(16):4503-4516, 2004b.

Gärdsback, Mattias and Tibert, Gunnar. Deployment control of spinning space webs. Journal of guidance, control, and dynamics, 32(1):40-50, 2009.

Garner, Charles, Diedrich, Benjamin, and Leipold, Manfred. A summary of solar sail technology developments and proposed demonstration missions. NASA Technical Report JPC-99-2697, 1999.

Goldstein, B.E., Buffington, A., Cummings, A.C., Fisher, R.R., Jackson, B.V., Liewer, P.C., Mewaldt, R.A., and Neugebauer, M. Solar polar sail mission: report of a study to put a scientific spacecraft in a circular polar orbit about the sun. In SPIE's International Symposium on Optical Science, Engineering, and Instrumentation, pages 65-76. International Society for Optics and Photonics, 1998.

Greschik, Gyula, Murphey, T, Mikulas, M, and Belvin, K. A rule of thumb for the suspension of film sheets without catenaries. In 44th AIAA Structures, Structural Dynamics, and Materials Conference, Norfolk, VA, 2003.

Guest, SD and Pellegrino, S. Analytical models for bistable cylindrical shells. Proceedings of the Royal Society A: Mathematical, Physical and Engineering Science, 462(2067):839-854, 2006.

Herzl, George G. Tubular spacecraft booms: Extendible, reel stored. NASA Monograph SP-8065, 1970.

Iqbal, K. and Pellegrino, S. Bi-stable composite shells. In Proc. 41st AIAA/ASME/ASCE/AHS/ASC Structures, Structural Dynamics, and Materials Conference and Exhibit, volume 3, 2000.

JAXA. Website, 2010. URL http://global.jaxa.jp/projects/sat/ikaros/. (Date accessed 01/06/2016).

Johnson, Les, Whorton, Mark, Heaton, Andy, Pinson, Robin, Laue, Greg, and Adams, Charles. Nanosail-d: A solar sail demonstration mission. Acta Astronautica, 68(5):571-575, 2011.

Katan, C. Nasa's next solar sail: Lessons learned from nanosail-d2. 26th Annual AIAA/USU Conference on Small Satellites, 2012.

Kessler, Donald J and Cour-Palais, Burton G. Collision frequency of artificial satellites: The creation of a debris belt. Journal of Geophysical Research: Space Physics (1978-2012), 83(A6): 2637-2646, 1978.

Lappas, Vaios, Adeli, Nasir, Visagie, Lourens, Fernandez, Juan, Theodorou, Theodoros, Steyn, Willem, and Perren, Matthew. Cubesail: A low cost cubesat based solar sail demonstration mission. Advances in Space Research, 48(11):1890-1901, 2011. 
Macdonald, M., Hughes, G.W., McInnes, CR, Lyngvi, A., Falkner, P., and Atzei, A. Geosail: an elegant solar sail demonstration mission. Journal of Spacecraft and Rockets, 44(4):784-796, 2007.

Mar, John and Garrett, Todd. Mechanical design and dynamics of the alouette spacecraft. Proceedings of the IEEE, 57(6):882-896, 1969.

McInnes, Colin R. Deflection of near-earth asteroids by kinetic energy impacts from retrograde orbits. Planetary and Space Science, 52(7):587-590, 2004a.

McInnes, Colin R, MacDonald, Malcolm, Angelopolous, Vassilis, and Alexander, David. Geosail: Exploring the geomagnetic tail using a small solar sail. Journal of Spacecraft and Rockets, 38 (4):622-629, 2001.

McInnes, C.R. Solar sailing: technology, dynamics and mission applications. Springer, $2004 \mathrm{~b}$.

NASA. Website, 2010. URL http://www.nasa.gov/images/content/475897. (Date accessed 04/03/2017).

Oberg, Erik, Jones, Franklin D, Horton, Holbrook L, Ryffel, Henry H, and Geronimo, James H. Machinery's handbook. Industrial Press, Incorporated, 2016.

Ogilvie, KW, Von Rosenvinge, T, and Durney, AC. International sun-earth explorer: a threespacecraft program. Science, 198(4313):131-138, 1977.

Ridenoure, Rex, Munakata, Riki, Diaz, Alex, Wong, Stephanie, Plante, Barbara, Stetson, Doug, Spencer, Dave, and Foley, Justin. Lightsail program status: one down, one to go. 29th Annual AIAA/USU Conference on Small Satellites, 2015.

Rimrott, F. P. J. Storable tubular extendible member a unique machine element. Journal of Machine Design, 37:156-165, 1965a.

Rimrott, FPJ. Two secondary effects in bending of slit thin-walled tubes. Jounal Applied Mechanics, 1965b.

Rimrott, FPJ. Stem self-extension velocities. Canadian Aeronautics and Space Journal, 13:1-7, 1967.

Rimrott, FPJ. Self-extension of an open section tube. Theory of shells, pages 495-508, 1980.

Seffen, KA and Pellegrino, S. Deployment of a rigid panel by tape-springs. CUED/D-STRUCT/TR $168,1997$.

Seffen, KA and Pellegrino, S. Deployment dynamics of tape springs. Proceedings of the Royal Society of London. Series A: Mathematical, Physical and Engineering Sciences, 455(1983):1003$1048,1999$.

Sickinger, C. and Herbeck, L. Deployment strategies, analyses and tests for the cfrp booms of a solar sail. In Proc. European Conf. on Spacecraft Structures, Materials and Mechanical Testing, 2002 .

Stohlman, Olive R and Lappas, VJ. Deorbitsail: a deployable sail for de-orbiting. 54th AIAA/ASME/ASCE/AHS/ASC Structures, Structural Dynamics, and Materials Conference, Boston, MA, 2013.

Stohlman, Olive R, Fernandez, J, Lappas, Vaios, Hillebrandt, Martin, Straubel, Marco, and Hühne, Christian. Testing of the deorbitsail drag sail subsystem. In Proceedings of the $54 A I A A / A S$ ME/ASCE/AHS/ASC Structures, Structural Dynamics, and Materials Conference, 2013. 
Su, Xiaofeng, Abdi, Frank, Taleghani, Barmac, and Blandino, Joseph. Wrinkling analysis of a kapton square membrane under tensile loading. In 44th AIAA/ASME/ASCE/AHS/ASC Structures, Structural Dynamics, and Materials Conference, page 1985, 2003.

Timoshenko, Stephen, Woinowsky-Krieger, Sergius, and Woinowsky-Krieger, S. Theory of plates and shells, volume 2. McGraw-hill New York, 1959.

Tsuda, Y., Mori, O., Funase, R., Sawada, H., Yamamoto, T., Saiki, T., Endo, T., and Kawaguchi, J. Flight status of ikaros deep space solar sail demonstrator. Acta Astronautica, 69(9):833-840, 2011.

Tsuda, Yuichi, Mori, Osamu, Funase, Ryu, Sawada, Hirotaka, Yamamoto, Takayuki, Saiki, Takanao, Endo, Tatsuya, Yonekura, Katsuhide, Hoshino, Hirokazu, and Kawaguchi, Jun'ichiro. Achievement of ikaros japanese deep space solar sail demonstration mission. Acta Astronautica, 82(2):183-188, 2013.

Upadhyaya, B.R. The self-extension velocity of overlapped tubular antenna when released by untwisting. Master's thesis, Department of Mechanical Engineering University of Toronto, 1968.

Viquerat, A, Schenk, M, Sanders, B, and Lappas, VJ. Inflatable rigidisable mast for end-of-life deorbiting system. In European Conference on Spacecraft Structures, Materials and Environmental Testing (SSMET), Apr, pages 1-4, 2014.

Viquerat, Andrew, Schenk, Mark, Lappas, Vaios, and Sanders, Berry. Functional and qualification testing of the inflatesail technology demonstrator. In 2nd AIAA Spacecraft Structures Conference, page 1627, 2015.

Walker, SJ and Aglietti, GS. Modeling the hinge moment of skew-mounted tape spring folds. Journal of Aerospace Engineering, 20(2):102-115, 2007a.

Walker, SJI and Aglietti, GS. A study of tape spring fold curvature for space deployable structures. Proceedings of the Institution of Mechanical Engineers, Part G: Journal of Aerospace Engineering, 221(3):313-325, 2007b.

Weisstein, Eric W. "Archemedes' Spiral" From MathWorld-A Wolfram Web Resource, March 2016. URL http://mathworld.wolfram.com/ArchimedesSpiral.html.

West, John L. The geostorm warning mission: enhanced opportunities based on new technology. 14th AAS/AIAA Space Flight Mechanics Conference, 2004.

West, John L and Derbès, Billy. Solar sail vehicle system design for the geostorm warning mission. AIAA-2000-5326, 5326:2000, 2000.

Yen, C.L. Solar sail geostorm warning mission design. 14th AAS/AIAA Space Flight Mechanics Conference, 2004.

Young, Warren Clarence and Budynas, Richard Gordon. Roark's formulas for stress and strain, volume 7. McGraw-Hill New York, 2002. 


\section{Chapter 8}

\section{Appendix A}

A second set of results for the first experimental rig is shown below.

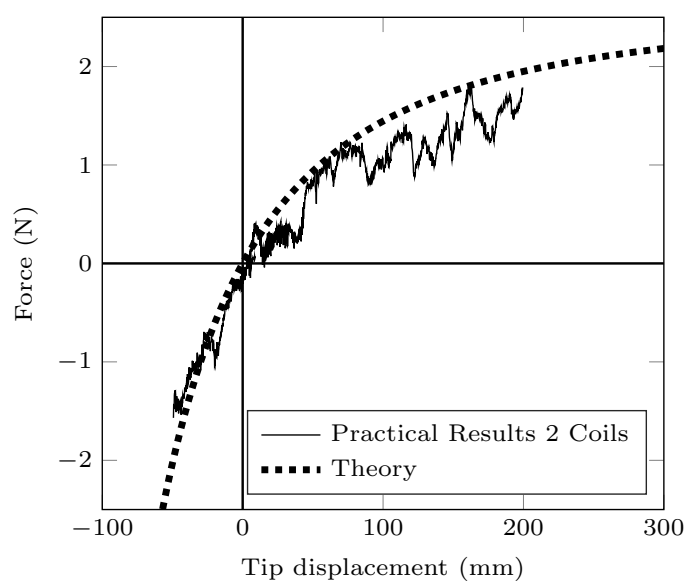

(a) 2 coils

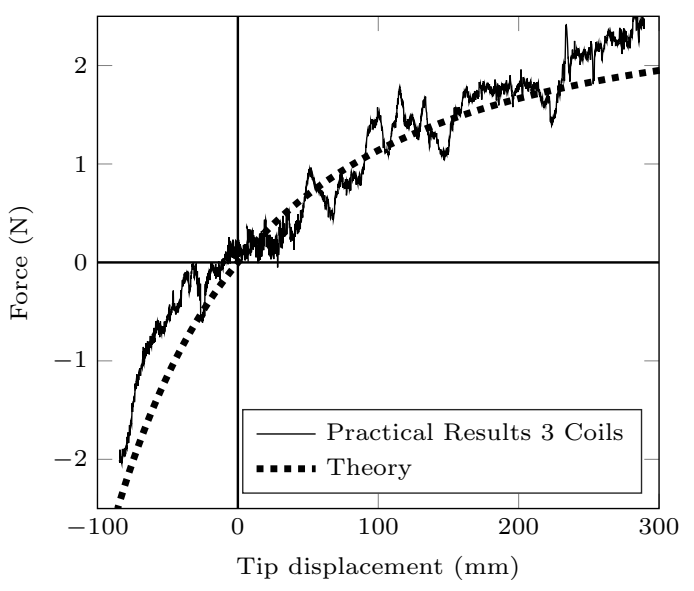

(b) 3 coils

Figure 8.1: Results for 2 and 3 coils. Boom dimensions are shown in Table 3.1.

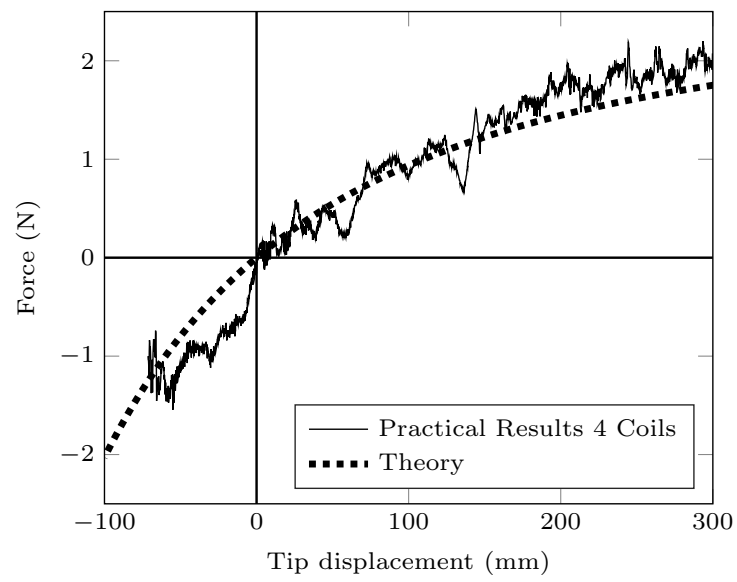

Figure 8.2: Results for 4 coils. Boom dimensions are shown in Table 3.1. 


\section{Chapter 9}

\section{Appendix B}

This appendix contains the MATLAB scripts used to display some of the figures within the thesis and gives more detail on the method used to find the tip force and central spindle torques including the spiral geometry of the coil and the effect of the compression springs.

\section{Strain energy plotted against coil radius}

This script displays the strain energy stored in the coil held at different radii. It uses the assumption that the coil radius is constant from the inside to the outside of the coil as a simplification. The script uses Equations 3.1 and 3.4. The script holds the bend angle constant but varies the length and radius of the coil as shown in Figure 9.1(b).

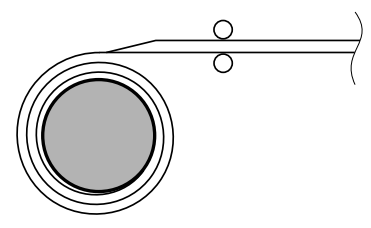

(a)

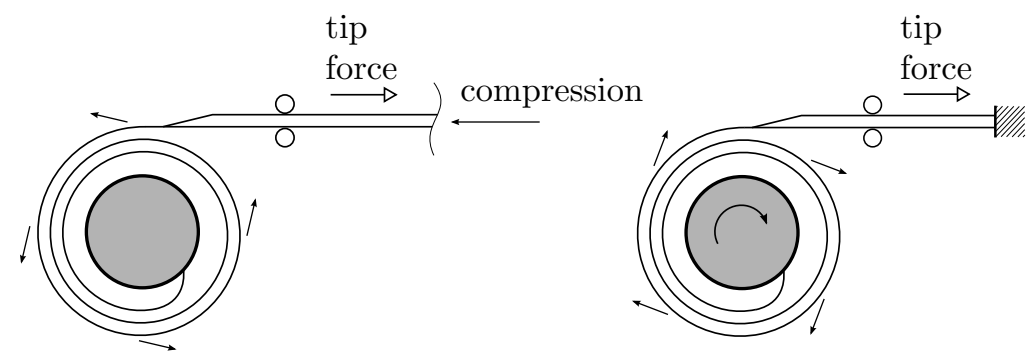

(b)

(c)

Figure 9.1: (a) The initial condition of a coiled boom with no tip force. (b) The central spindle is held fixed and the boom tip is compressed towards the central spindle. The coil expands to a radius which is larger than the natural radius of the tape spring, this creates the tip force. The tip force is shown with an arrow with a large head, the rest of the arrows represent the boom motion. (c) The boom tip is held stationary, the central spindle turns and the coil unwinds. The coil radius is larger than the natural radius of the tape spring, creating the tip force. This motion is a closer representation of blossoming.

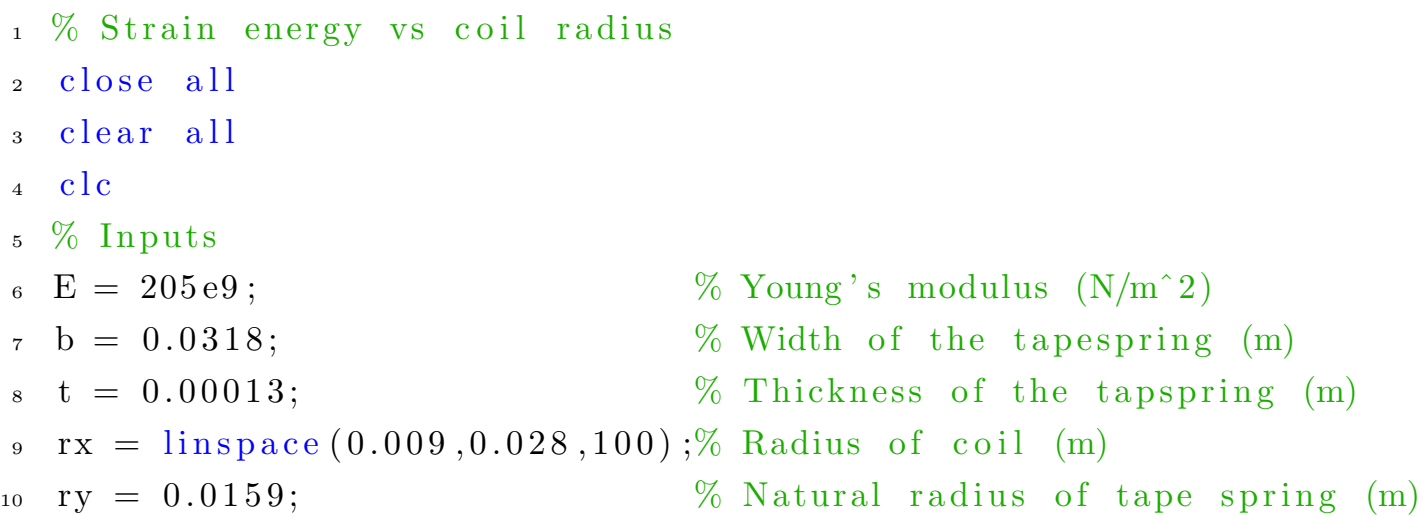




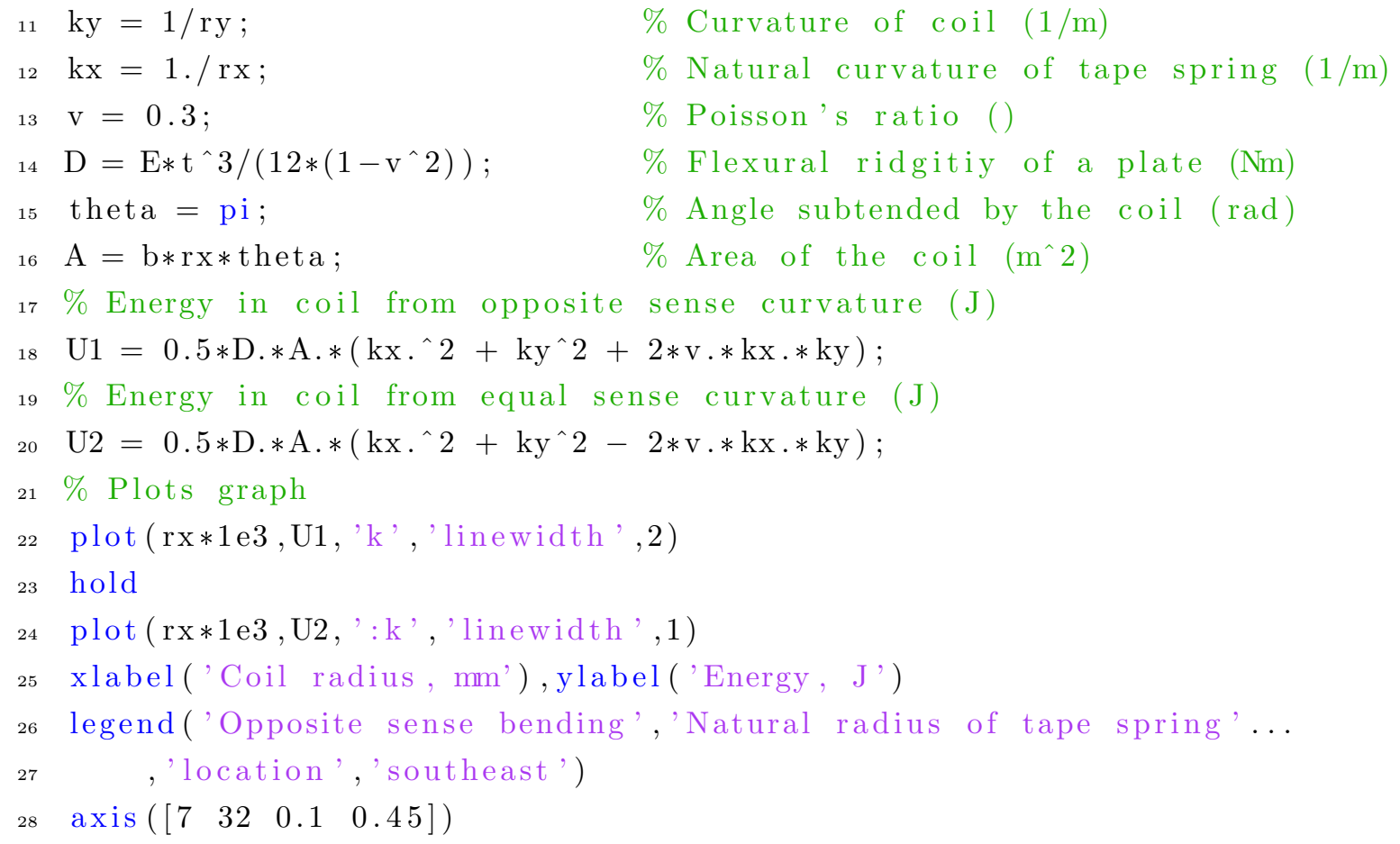

\section{Tip force plotted against length compressed}

This script displays the tip force when a boom is displaced. It uses the assumption that the coil radius is constant from the inside to the outside of the coil as a simplification. The script uses Equation 3.12 to find the tip force. The script holds the bend angle constant but varies the length and radius of the coil as shown in Figure 6.1(b).

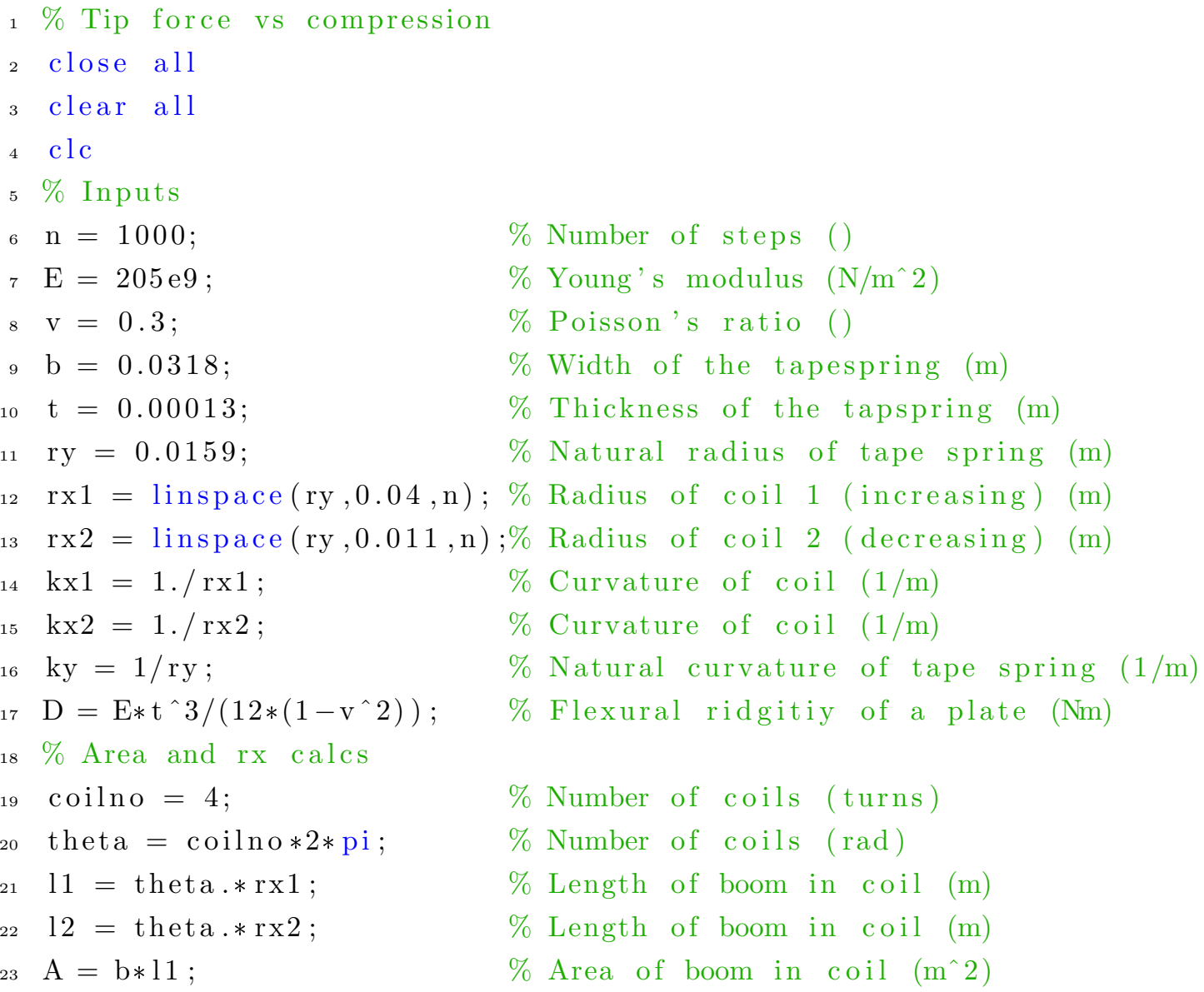




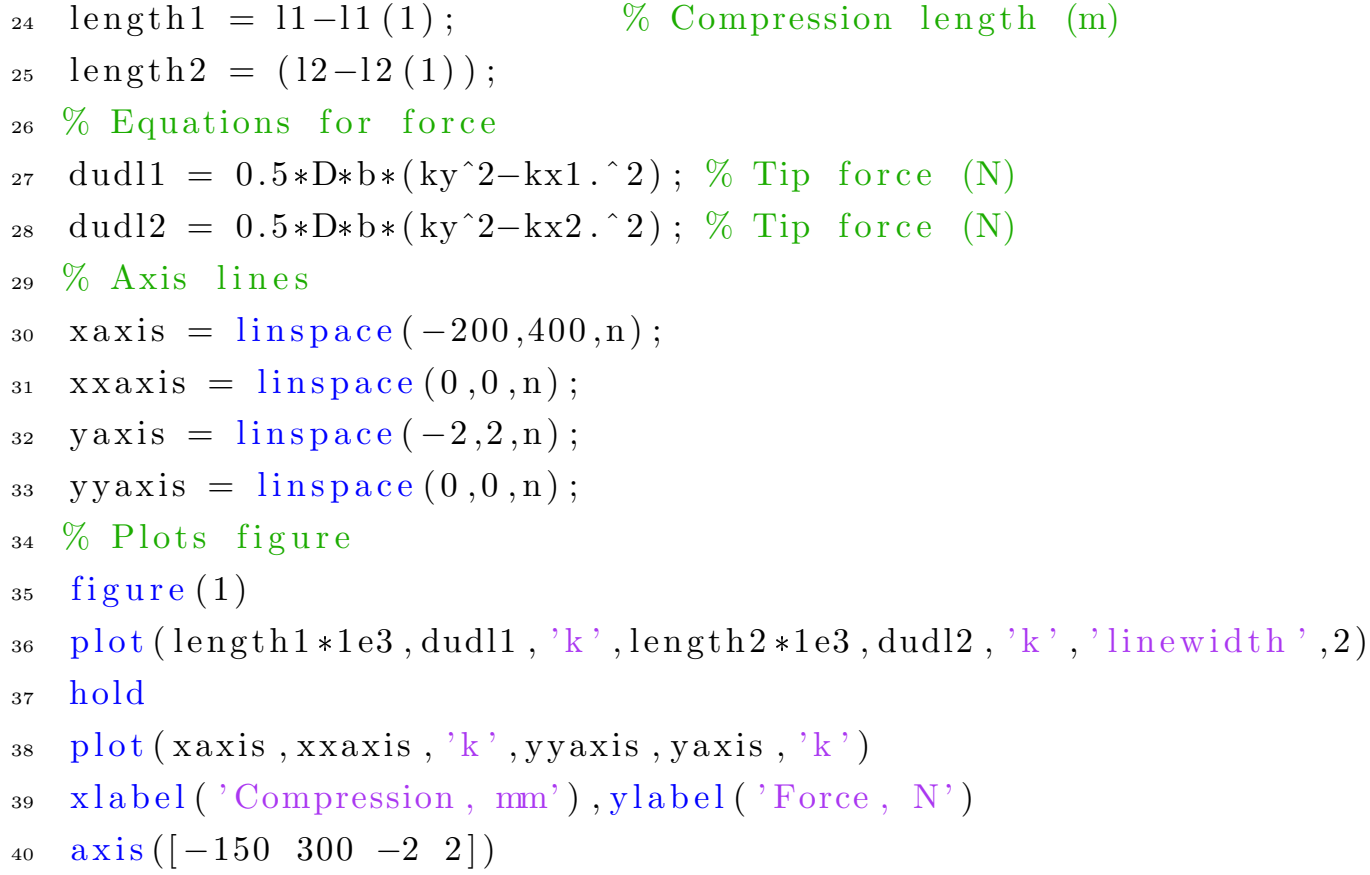

\section{Strain energy comparison plotted against coil radius}

This script compares the strain energy stored in the tape spring at different coil radii for spiral geometry and the simplification of the coil geometry to a circle. It uses the scripts mycoillength and coilenergy. The script holds the bend angle constant but varies the length and radius of the coil as shown in Figure 6.1(b).

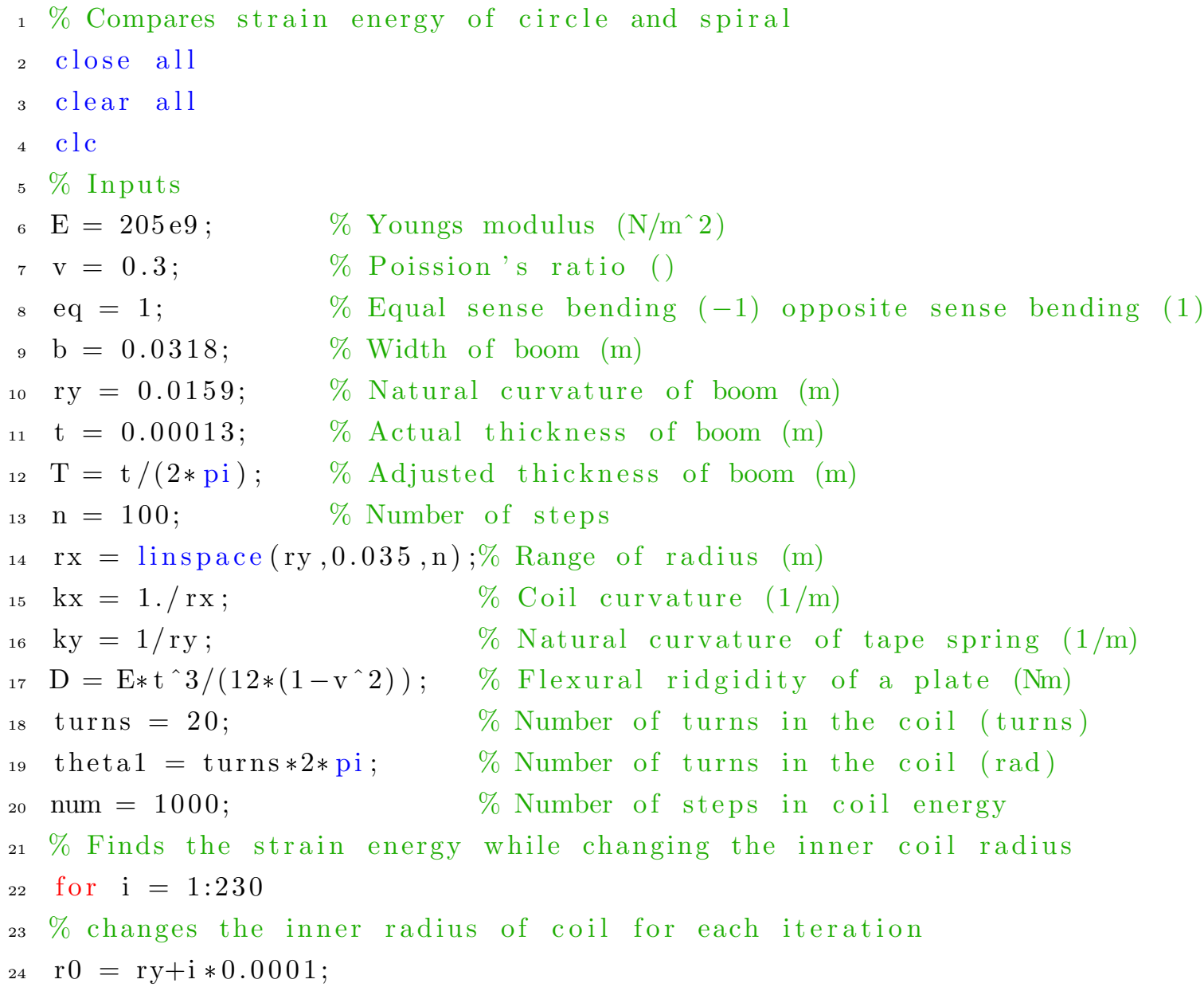




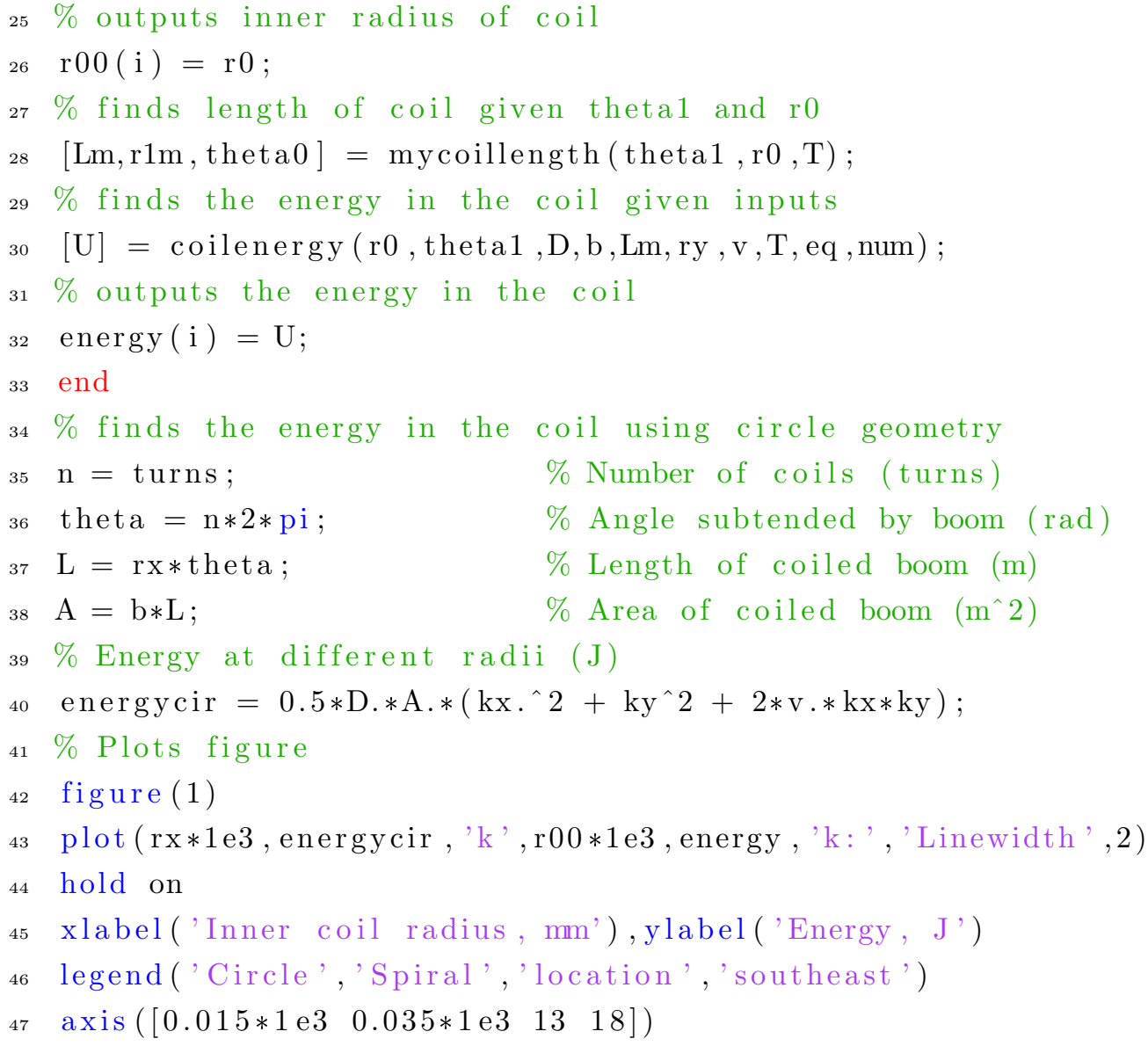

\section{Tip force comparison plotted against coil radius}

This script compares the tip force at different coil radii for spiral geometry and the simplification of the coil geometry to a circle. It uses the scripts coillength coilenergy and springenergy. The script holds the bend angle constant but varies the length and radius of the coil as shown in Figure 6.1(b). Within the script there is a double 'for' loop. The outside loop changes the angle subtended for each step. The inside 'for' loop then holds the coil angle constant but changes the length and coil radius to find the strain energies stored in the coil and the compression springs. The gradient of the strain energies gives the tip force.

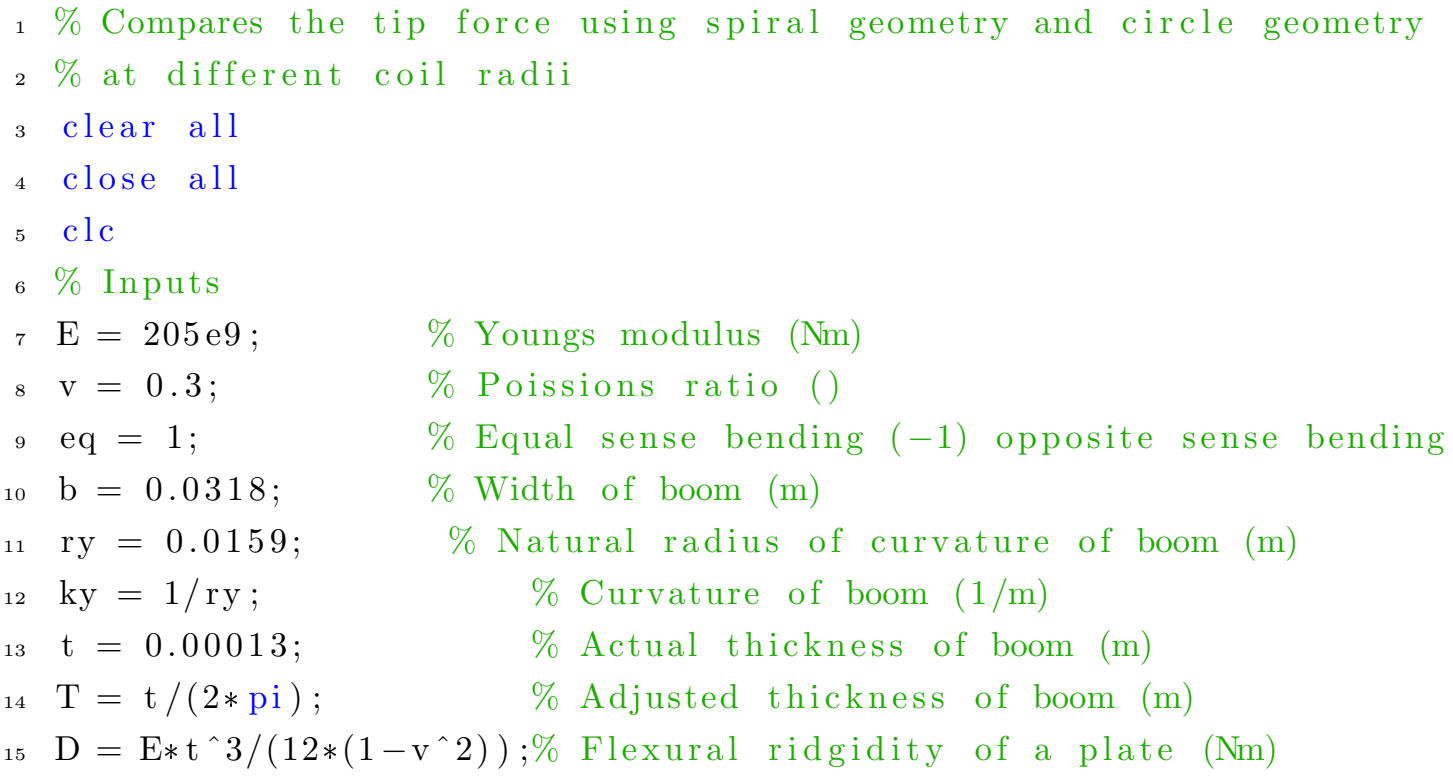




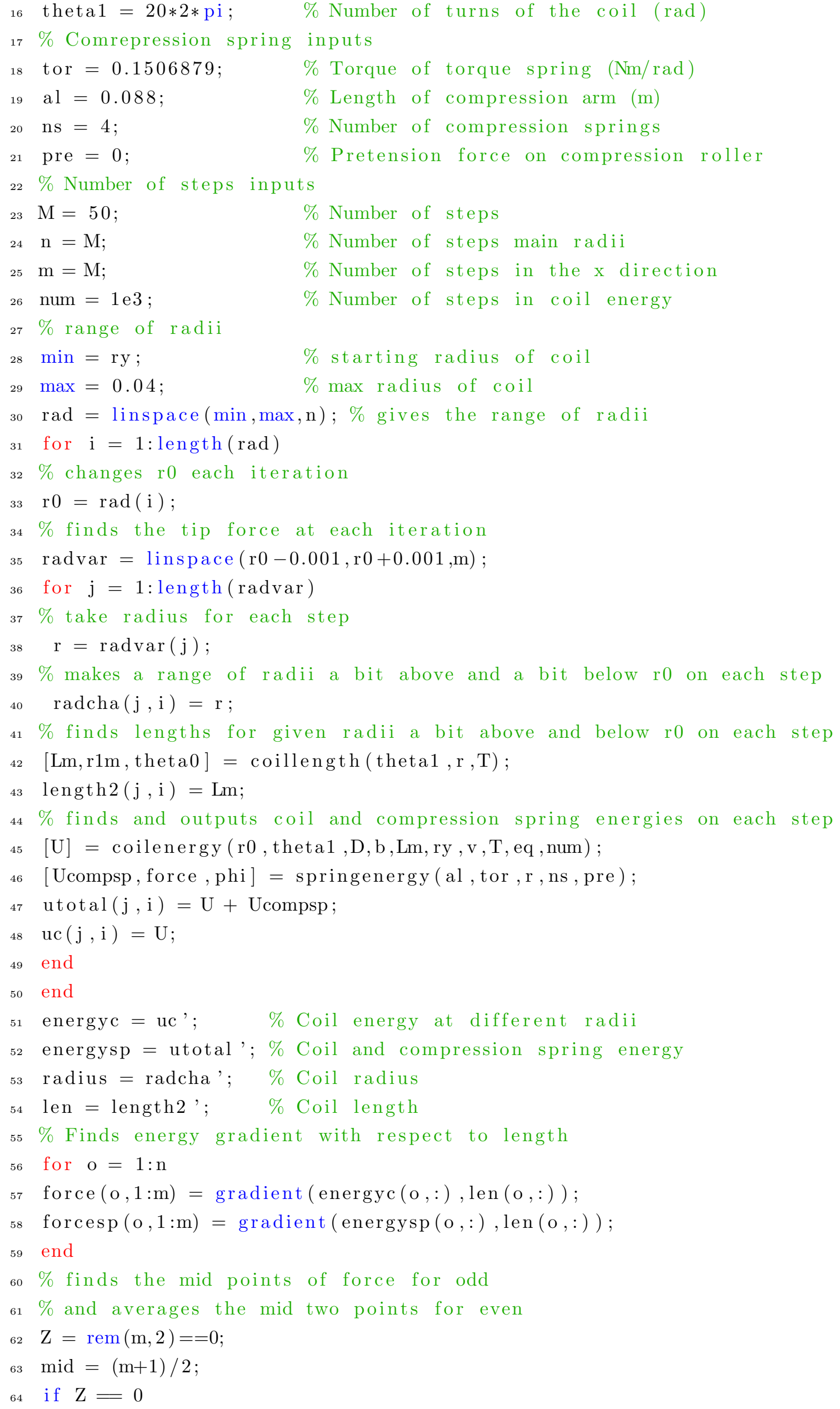




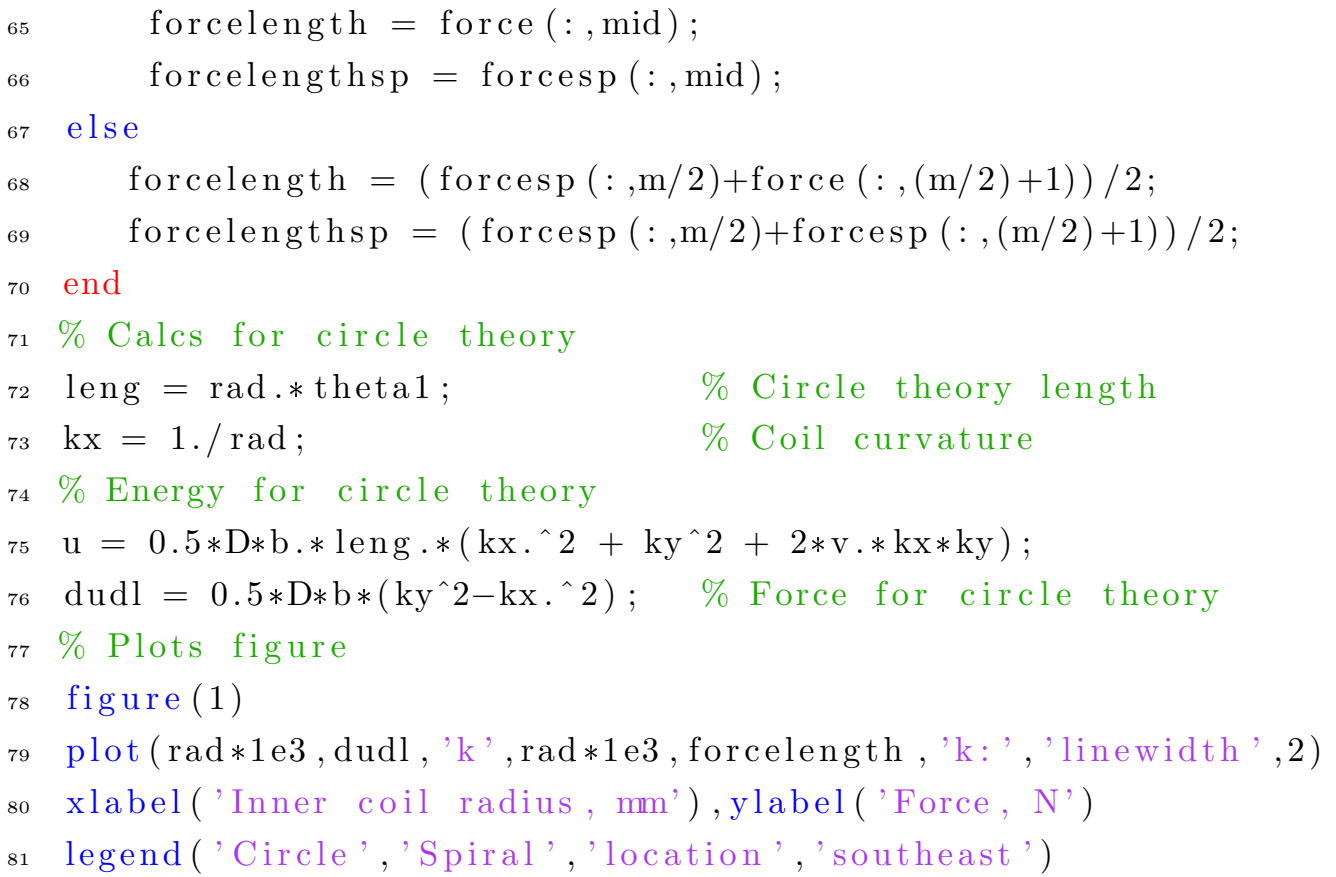

\section{Tip force with compression springs plotted against angle sub- tended by coil}

This script displays the tip force including the effect of the compression springs against the number of turns in the coil. It uses the scripts coillength, inversecoillength, coilenergy and springenergy. The script holds the length constant but decreases the coil angle and increases the coil radius as shown in Figure 6.1(c). Within the script there is a double 'for' loop. The outside loop changes the angle subtended for each step. The inside 'for' loop then holds the coil angle constant but changes the length and coil radius to find the strain energies stored in the coil and the compression springs. The gradient of the strain energies gives the tip force.

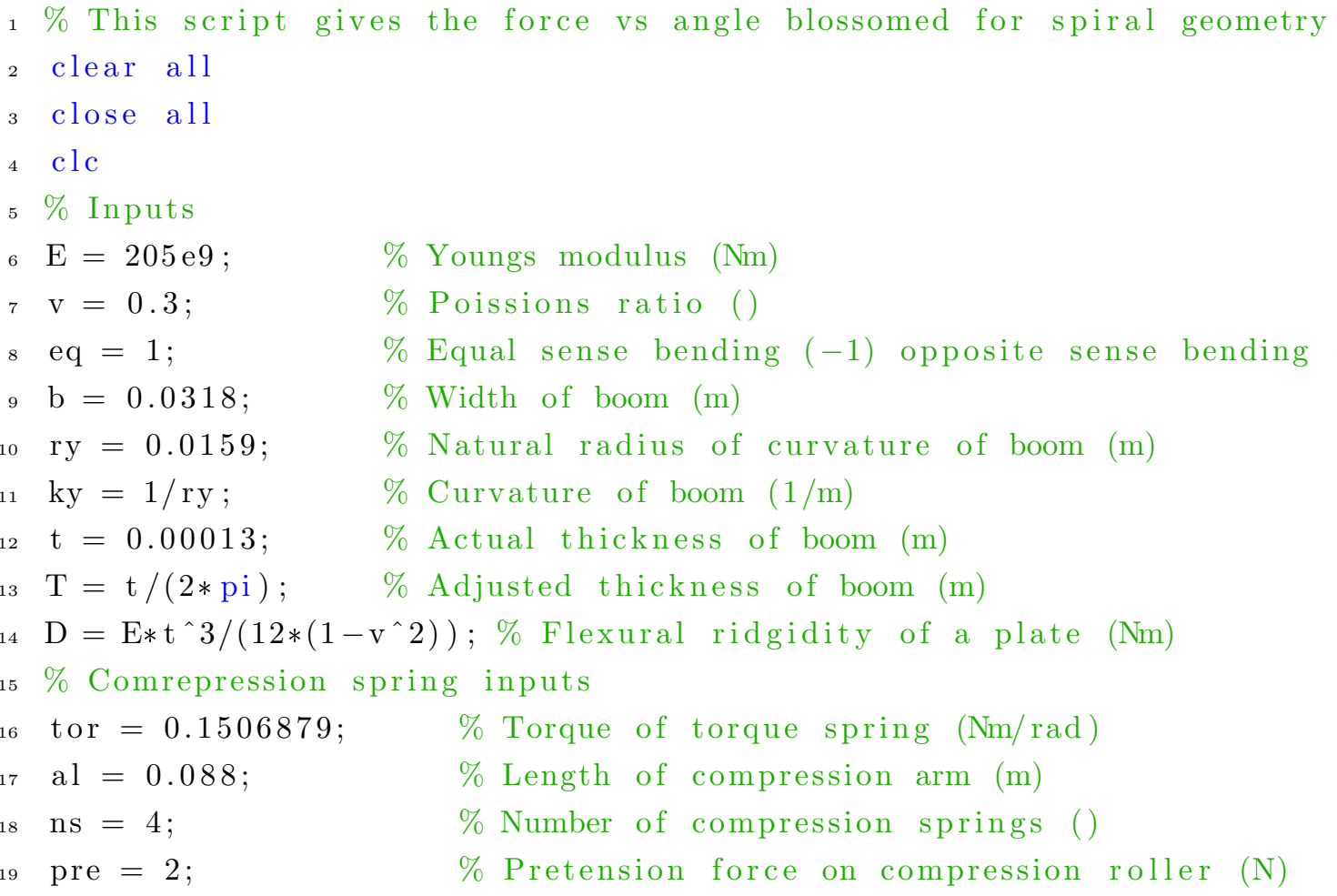




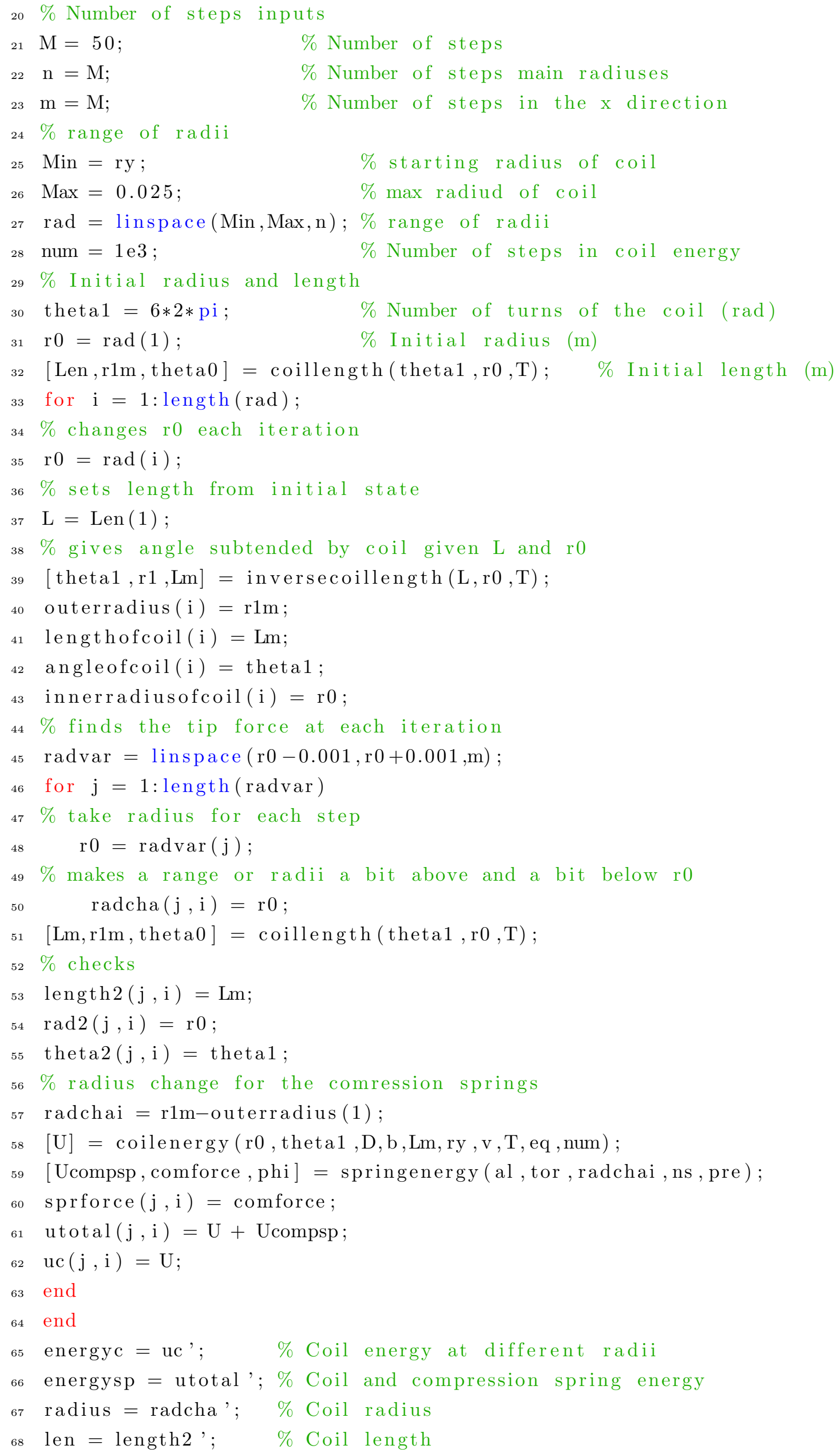




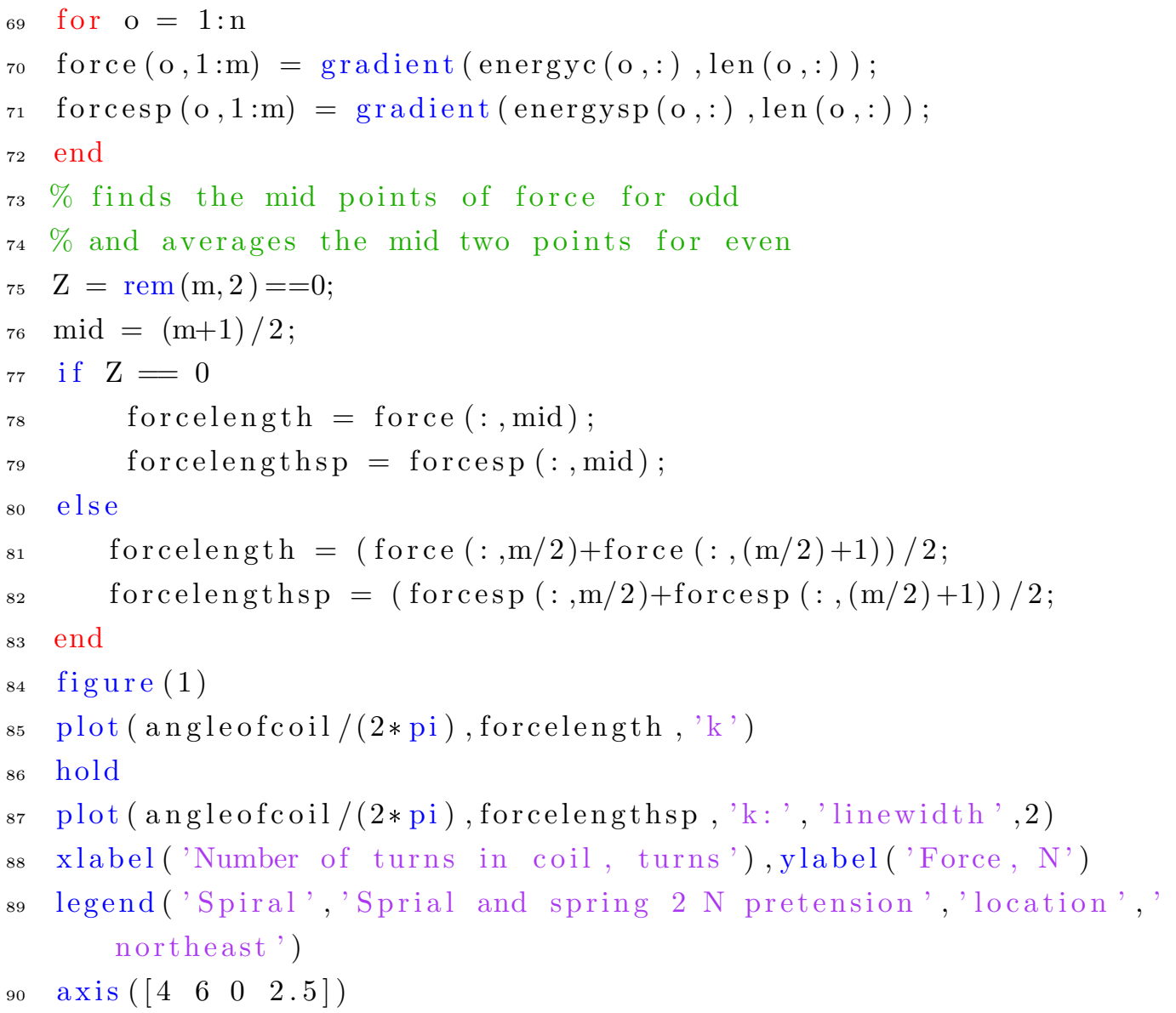

\section{Torque comparison}

This script compares the central spindle torque and self deployment torque for a tape spring in opposite sense bending. The script holds the length constant but decreases the coil angle and increases the coil radius as shown in Figure 6.1(c).

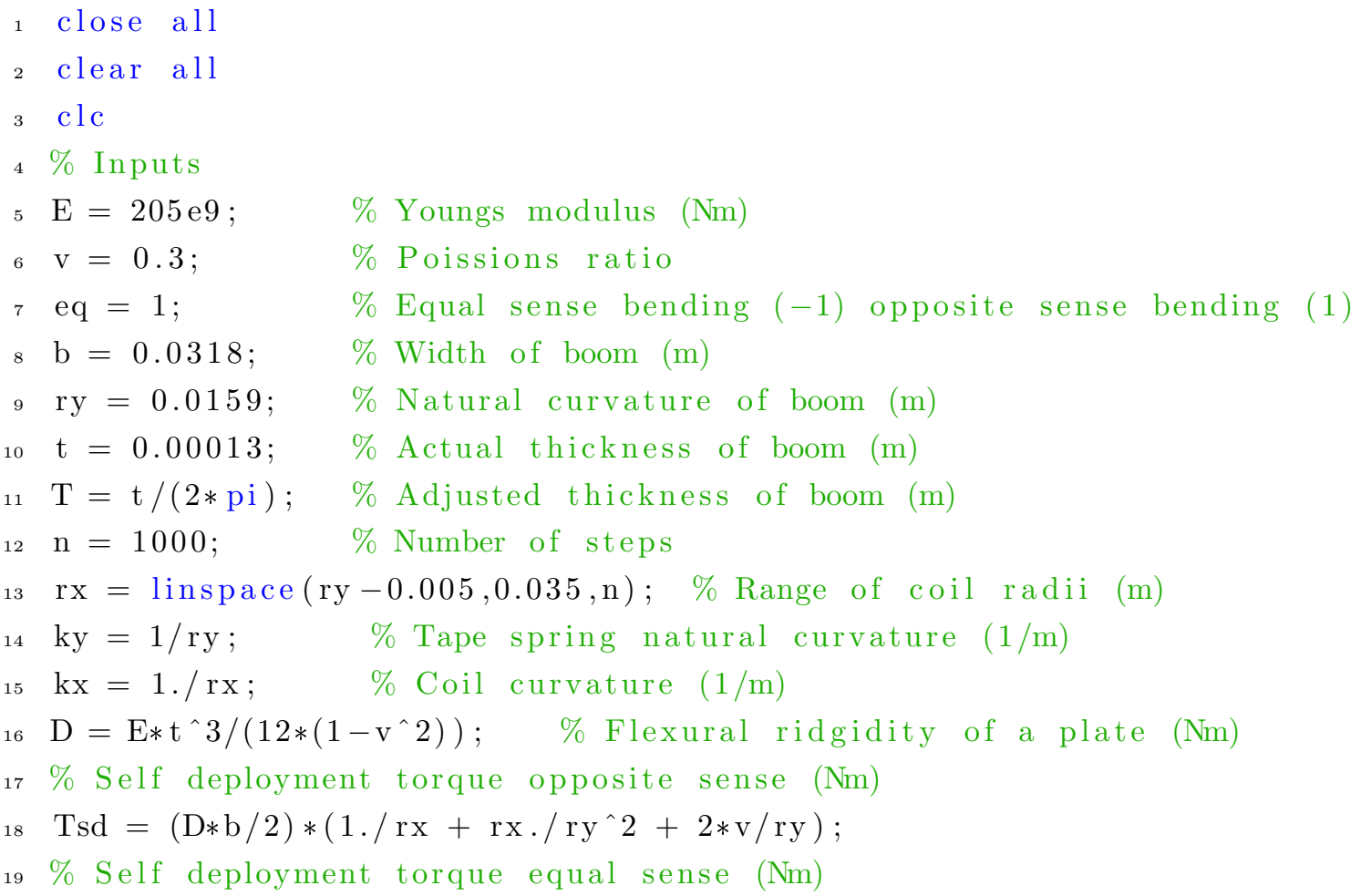




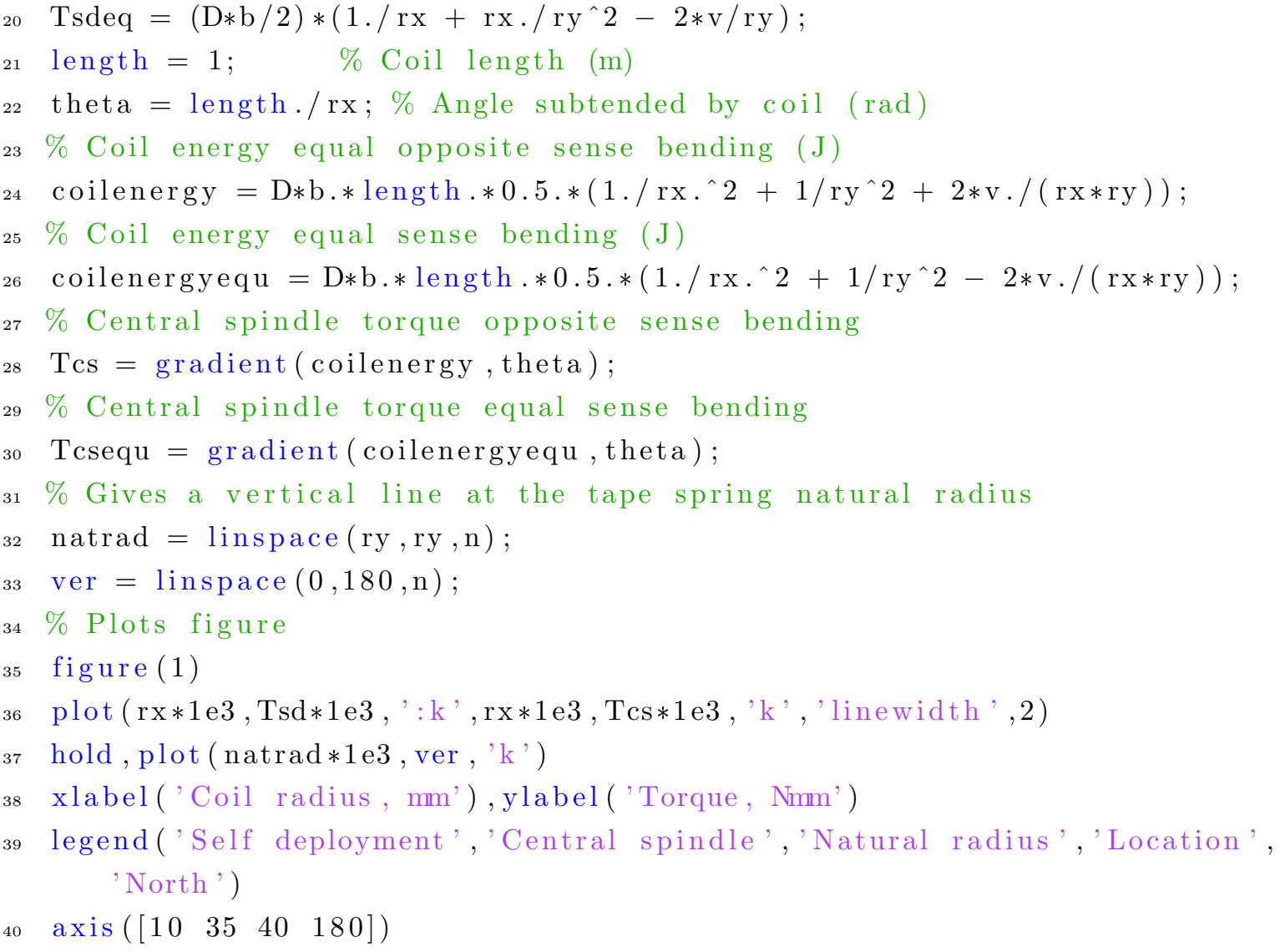

\section{Tip force and torque}

This script compares the tip force and central spindle torque to practical results. It also incorporates the friction model. It uses the scripts coillength, inversecoillength, coilenergy and springenergy. The script holds the length constant but decreases the coil angle and increases the coil radius as shown in Figure 6.1(c).

$1 \%$ This script gives the tip force and central spindle torque

$2 \%$ vs angle blossomed for spiral geometry

3 clear all

4 close all

$5 \mathrm{clc}$

$6 \%$ Inputs

$7 \mathrm{E}=205 \mathrm{e} 9 ; \quad \%$ Youngs modulus (Nm)

$8 \mathrm{v}=0.3 ; \quad \%$ Poissions ratio ()

9 eq $=1 ; \quad \%$ Equal sense bending $(-1)$ opposite sense bending (1)

$10 \mathrm{nb}=2 ; \quad \%$ Number of booms ()

$11 \mathrm{~b}=0.0254 ; \quad \%$ Width of boom $(\mathrm{m})$

12 ry $=35 * 1 \mathrm{e}-3 / 2 ; \%$ Natural radius of boom (m)

$13 \mathrm{ky}=1 / \mathrm{ry} ; \quad \%$ Curvature of boom $(1 / \mathrm{m})$

$14 \mathrm{t}=0.00013 ; \quad \%$ Actual thickness of boom $(\mathrm{m})$

$15 \mathrm{gt}=\mathrm{t} * \mathrm{nb} ; \quad \%$ Geometry thickness (to allow for the number of booms)

${ }_{16} \mathrm{~T}=\mathrm{gt} /(2 * \mathrm{pi}) ; \%$ Adjusted geometry thickness of boom (m)

${ }_{17} \mathrm{D}=\mathrm{E} * \mathrm{t} \wedge 3 /\left(12 *\left(1-\mathrm{v}^{\wedge} 2\right)\right) ; \quad \%$ Flexural ridgidity of a plate $(\mathrm{Nm})$

$18 \mathrm{mu}=0.25 ; \quad \%$ Coefficient of friction between two layers ()

$19 \%$ Comrepression spring inputs 


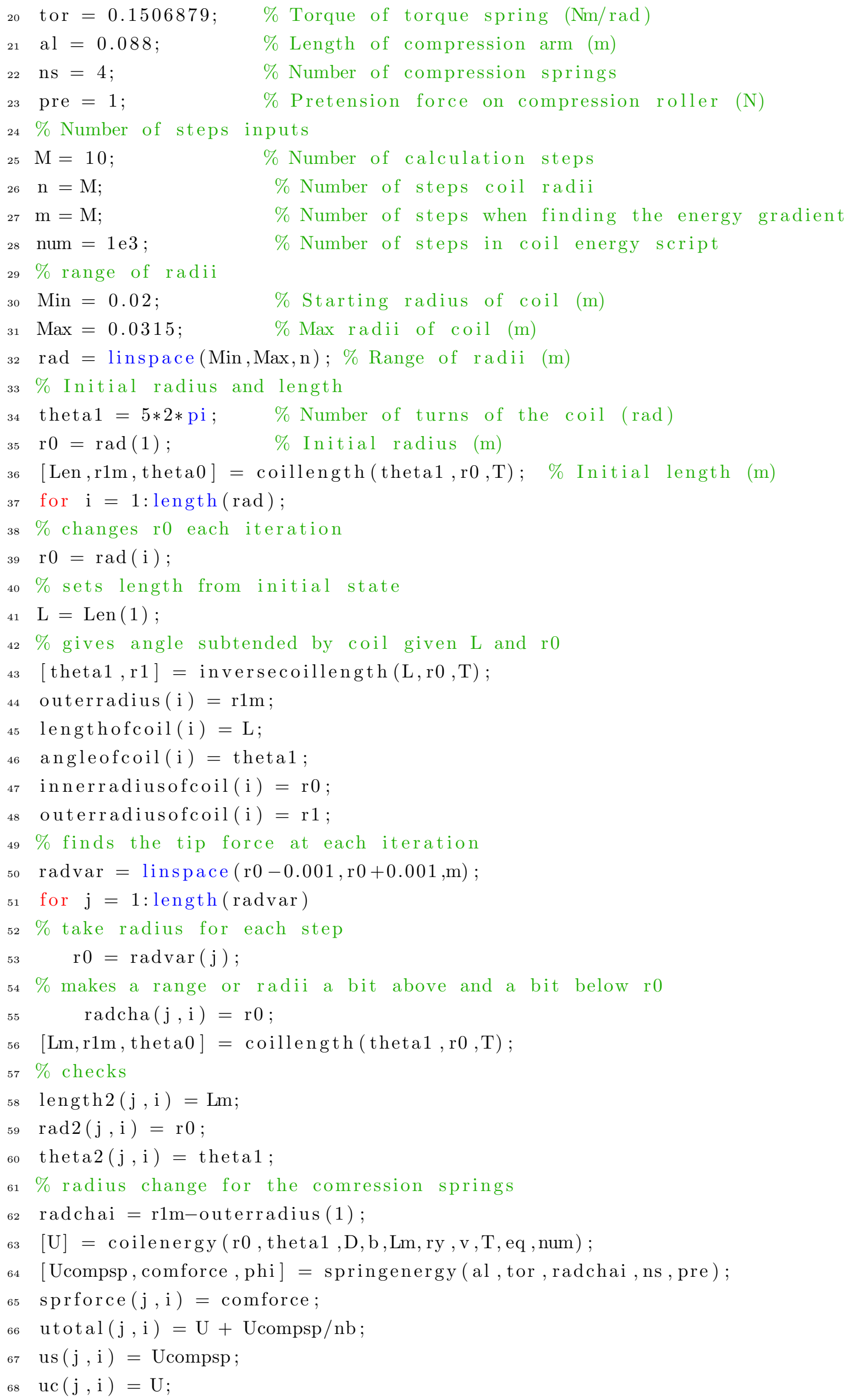




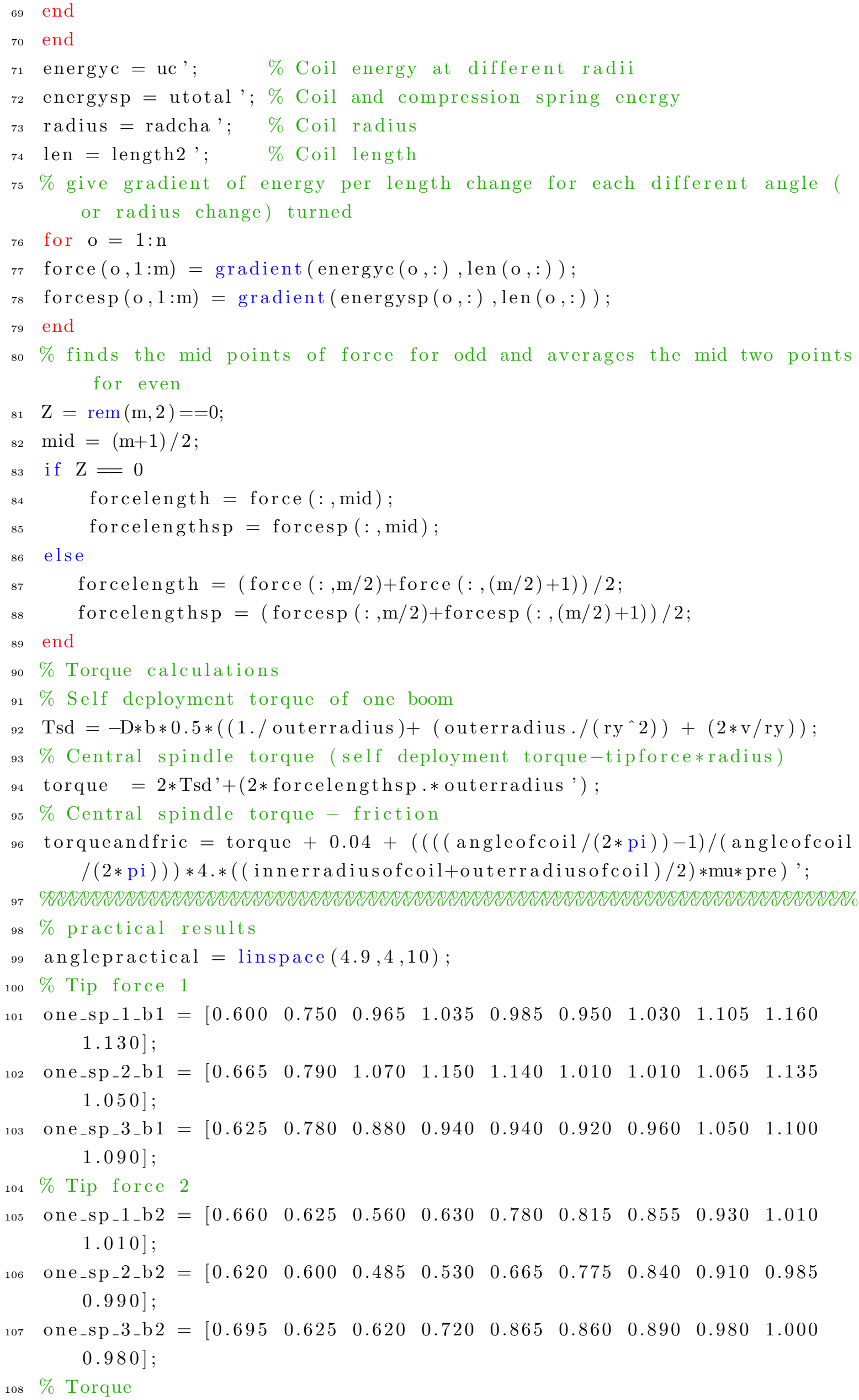




\section{coilenergy}

This script is used in some of the other scripts. It finds the strain energy stored in the coil. The inputs are: the inner coil radius, the angle subtended by the coil, the flexural rigidity of the tape spring, the width of the tape spring, the coil length, the tape springs natural cross section radius, the thickness of the tape spring, whether the tape spring is in equal or opposite sense bending and the number of calculation steps to take. The output is the strain energy stored in the tape spring.

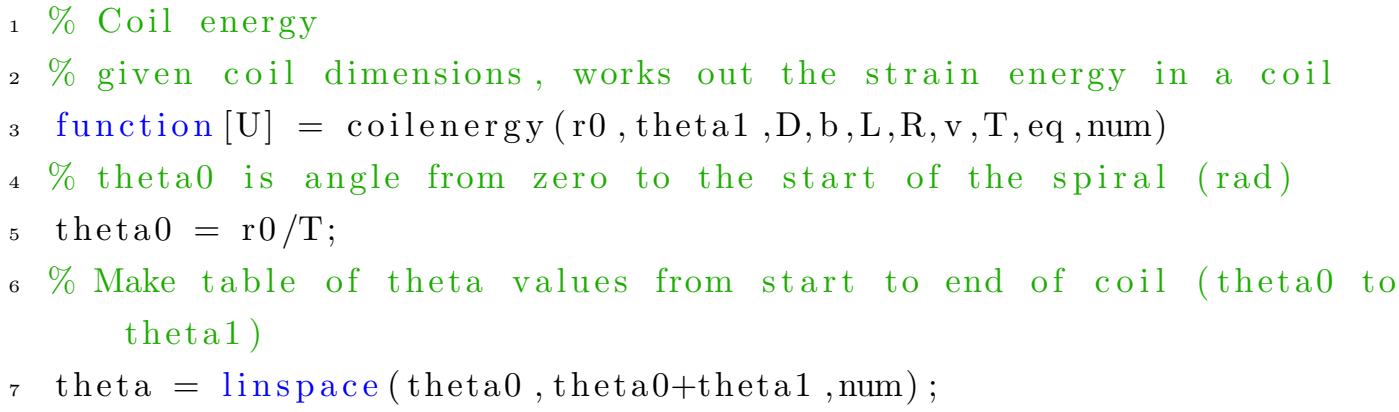


$8 \%$ Makes a table for the lengths of boom given theta

$9 \mathrm{~s}=0.5 * \mathrm{~T} *\left(\left(\right.\right.$ theta. $*(1+$ theta.^ 2$\left.) \cdot{ }^{\wedge} 0.5\right)+\operatorname{asinh}($ theta $\left.)\right) ;$

$10 \%$ Makes a table for the curvature $(\mathrm{K})$ of boom for given theta

${ }_{11} \mathrm{~K}=(2+$ theta.^2$) \cdot /\left(\mathrm{T} *(1+\right.$ theta.^ 2$\left.) \cdot{ }^{\wedge}(3 / 2)\right)$;

$12 \%$ Makes a table the radii for a given theta

$13 \mathrm{r}=1 . / \mathrm{K}$;

14 length1 $=\min (\mathrm{s}) ; \%$ Length of start of coil

15 length $2=\max (\mathrm{s}) ; \quad \%$ Length of end of coil

16 lengthofcoil = length2-length1; \% Length of coil we are intersted in

17 length $3=$ s-length1; \% Table of numbers of length of coil we are

interested in ( 0 to end of coil)

${ }_{8} \%$ Finds the area under the curvature vs length graph

$19 \mathrm{z}=\operatorname{trapz}($ length $3, \mathrm{~K})$;

${ }_{20} \%$ Gives area under the curve squared and divided by L

${ }_{21} \mathrm{Z}=\mathrm{z}^{\wedge} 2 / \mathrm{L}$

$22 \%$ New updated strain energy in the coil

${ }_{23} \mathrm{U}=(\mathrm{D} * \mathrm{~b} / 2) *\left((\mathrm{Z})+\left(\mathrm{L} / \mathrm{R}^{\wedge} 2\right)+(\mathrm{eq} * 2 * \mathrm{v} * \mathrm{z} \cdot /(\mathrm{R}))\right)$;

24 end

\section{inversecoillength}

This script is used in some of the other scripts. It finds the angle subtended by the coil. The inputs are: the length of the coil, the inner radius and the thickness of the tape spring. The outputs are the angle subtended by the coil, the outer radius of the coil and the length of the coil.

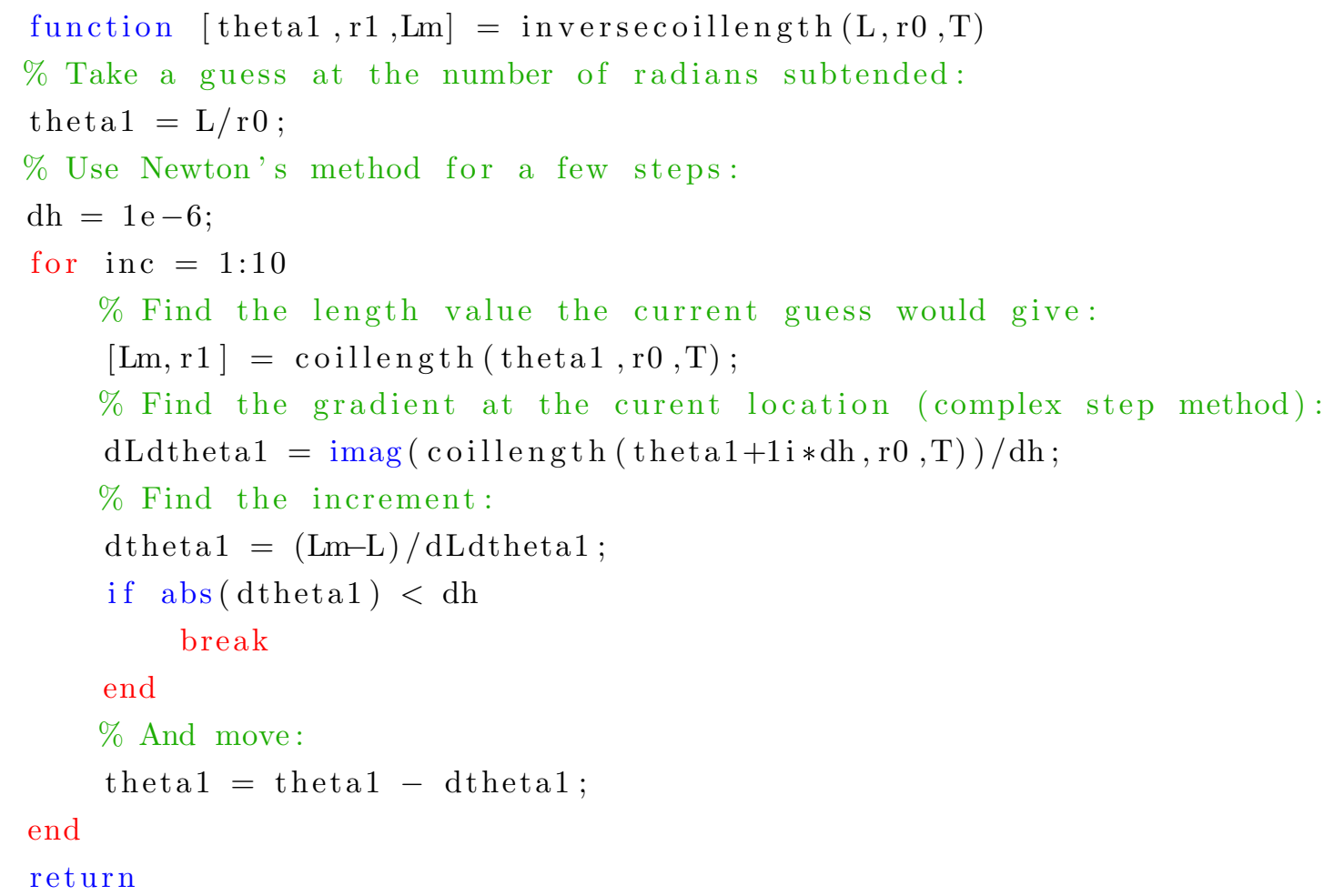

\section{coillength}

This script is used in some of the other scripts. It finds the coil length. The inputs are: the angle subtended by the coil, the inner radius and the thickness of the tape spring. The outputs are the 
length of the tape spring, the outer radius of the coil and the angle subtended from a zero position to the start of the coil.

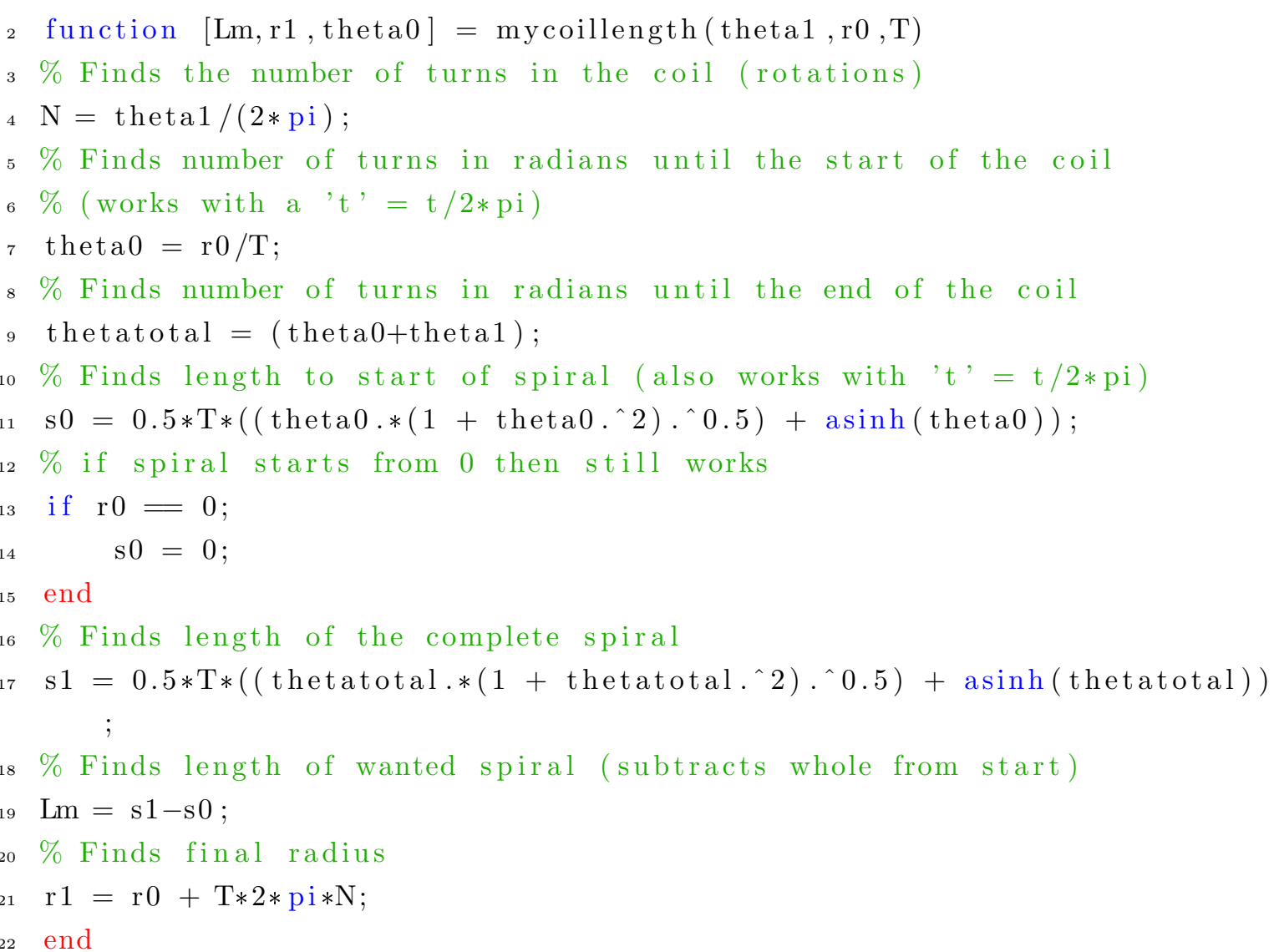

\section{springenergy}

This script is used in some of the other scripts. It finds the strain energy stored in the compression springs. The inputs are: the compression roller arm length, the torsional stiffness of the spring, the number of compression arms and the compression roller pretension force. The outputs are the strain energy stored in the compression spring, the force at the compression roller and the angle turned from the zero position by the spring.

2 function [Ucompsp, force,phi] = springenergy (al, tor, rr , ns, pre)

${ }_{3} \%$ Gives an initial torque from the pretensioned force applied to the roller

${ }_{4}$ tori $=$ pre*al;

$5 \%$ Gives a prestressed angle of the spring from the above found torque ( $\mathrm{rad}$ )

${ }_{6}$ preang $=$ tori $/$ tor ;

$7 \%$ Angle of displacement $=$ radius / arm length ( rad)

8 phi $=$ preang $+(\mathrm{rr} / \mathrm{al})$;

$9 \%$ Energy is $0.5 *$ spring torsional stiffness * angle of displacement ^2

10 Ucompsp $=\mathrm{ns} * 0.5 * \operatorname{tor} * \mathrm{phi}^{\wedge} 2$;

11 force $=$ tor $*$ phi $/$ al ;

12 end 